\title{
DC, RF, AND THERMAL CHARACTERIZATION OF HIGH ELECTRIC FIELD INDUCED DEGRADATION MECHANISMS IN GAN-ON-SI HIGH ELECTRON MOBILITY TRANSISTORS
}

\author{
A Thesis \\ presented to \\ the Faculty of California Polytechnic State University, \\ San Luis Obispo
}

\author{
In Partial Fulfillment \\ of the Requirements for the Degree \\ Master of Science in Electrical Engineering
}

by

Matthew Anthony Bloom

March 2013 
(C)2013

Matthew Anthony Bloom

ALL RIGHTS RESERVED 
TITLE:

AUTHOR:

DATE SUBMITTED:

COMMITTEE CHAIR:

COMMITTEE MEMBER:

COMMITTEE MEMBER:

COMMITTEE MEMBER:
DC, RF, and Thermal Characterization of High

Electric Field Induced Degradation Mechanisms in GaN-on-Si High Electron Mobility Transistors

Matthew Anthony Bloom

March 2013

Dr. Dennis Derickson, Department Chair Electrical Engineering Department California Polytechnic State University, San Luis Obispo

Dr. Vladimir Prodanov, Assistant Professor Electrical Engineering Department California Polytechnic State University, San Luis Obispo

Dr. Tina Smilkstein, Assistant Professor Electrical Engineering Department California Polytechnic State University, San Luis Obispo

Dr. Todd Weatherford, Associate Professor Naval Postgraduate School

Monterey, California 


\begin{abstract}
DC, RF, and Thermal Characterization of High Electric Field Induced Degradation

Mechanisms in GaN-on-Si High Electron Mobility Transistors
\end{abstract}

\title{
Matthew Anthony Bloom
}

Gallium Nitride (GaN) high electron mobility transistors (HEMTs) are becoming increasingly popular in power amplifier systems as an alternative to bulkier vacuum tube technologies. GaN offers advantages over other III-V semiconductor heterostructures such as a large bandgap energy, a low dielectric constant, and a high critical breakdown field. The aforementioned qualities make $\mathrm{GaN}$ a prime candidate for high-power and radiation-hardened applications using a smaller form-factor. Several different types of semiconductor substrates have been considered for their thermal properties and costeffectiveness, and Silicon ( $\mathrm{Si}$ ) has been of increasing interest due to a balance between both factors.

In this thesis, the DC, RF, and thermal characteristics of GaN HEMTs grown on Si-substrates will be investigated through a series of accelerated lifetime experiments. A figure of merit known as the critical voltage is explored and used as the primary means by which the GaN-on-Si devices are electrically strained. The critical voltage is defined as the specific voltage bias by which a sudden change in device performance is experienced due to a deformation of the target GaN HEMT's epitaxial structure. Literature on the topic details the inevitable formation of pits and cracks localized under the drain-side of the gate contact that promote electrical degradation of the devices via the inverse piezoelectric effect. Characteristic changes in device performance due to high field strain are recorded and physical mechanisms behind observed degraded performance are investigated.

The study assesses the performance of roughly $60 \mathrm{GaN}-$ on-Si HEMTs in four experimental settings. The first experiment investigates the critical voltage of the device in the off-state mode of operation and explores device recovery post-stress. The second experiment analyzes alterations in DC and RF performance under varying thermal loads and tracks the dependence of the critical voltage on temperature. The third experiment examines electron trapping within the HEMTs as well as detrapping methodologies. The final experiment links the changes in RF performance induced by high field strain to the small-signal parameters of the HEMT. Findings from the research conclude the existence of process-dependent defects that originate during the growth process and lead to inherent electron traps in unstressed devices. Electron detrapping due to high electric field stress applied to the HEMTs was observed, potentially localized within the AlGaN layer or GaN buffer of the HEMT. The electron detrapping in turn contributed to drain current recovery and increased unilateral performance of the transistor in the RF regime. Thermal experiments resulted in a positive shift in critical voltage, which enhanced gate leakage current at lower gate voltage drives. 


\section{ACKNOWLEDGMENTS}

I would like to first and foremost thank Dr. Dennis Derickson for giving me the opportunity to work on this research initiative. Without Dr. Derickson's guidance and mentorship through the entirety of the project, the results of the research would not have come to full fruition. I would also like to thank Jamie Carmo for providing the necessary tools and materials to successfully perform the experiments.

I would also like to thank Dr. Todd Weatherford and Matthew Porter of the Naval Postgraduate School in Monterey for their outstanding collaborative efforts with Cal Poly. The guidance and support from both Dr. Weatherford and Matthew Porter helped facilitate the research initiative and provide great insight into the realm of $\mathrm{GaN}$ technology.

I would like to thank Dr. Vladimir Prodanov for providing insight into issues I was experiencing during the initial stages of the DC studies and for becoming a part of my committee. I would also like to thank Dr. Tina Smilkstein for joining my committee on such a short notice.

I would like to thank all of my peers that have stuck by me during the whole research process and given me helpful suggestions along the way. Most importantly, I would like to thank my family for their everlasting support during all of my trials and tribulations. Without them, I would not be where I am today, and for that I am truly grateful. 


\section{TABLE OF CONTENTS}

Page

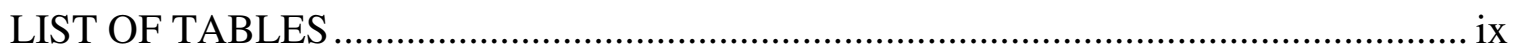

LIST OF FIGURES …………………………………........................................

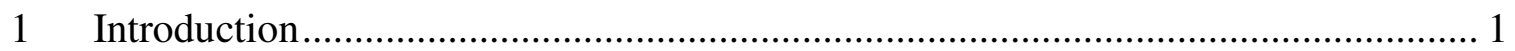

$1.1 \quad$ High Electron Mobility Transistors (HEMTs) ……………………………….... 1

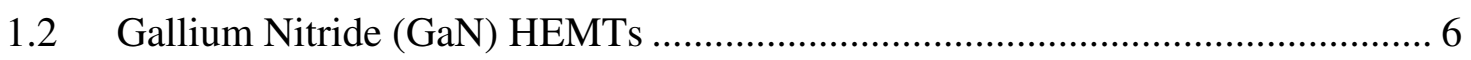

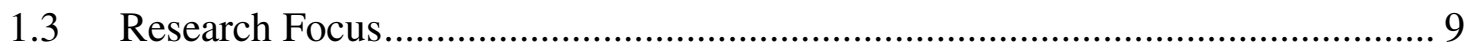

2 Device Under Test (DUT) Overview and Test Plan................................................. 11

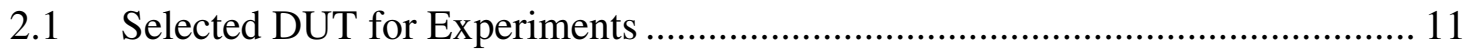

2.2 Simulation of the HEMT's DC and RF Characteristics ................................. 16

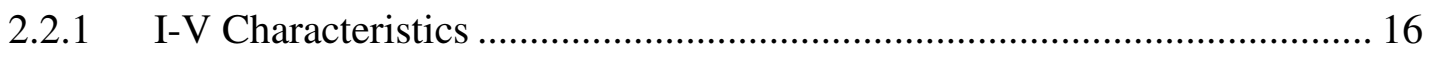

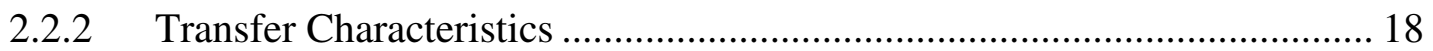

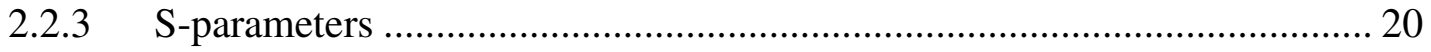

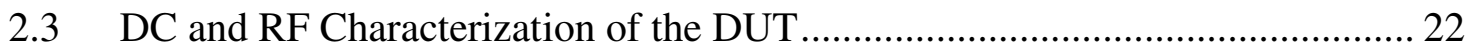

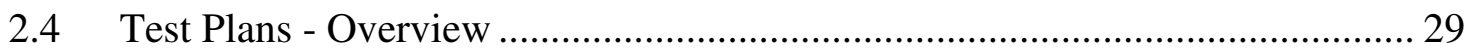

3 Experiment 1: High Electric Field Stress Analyses ................................................. 32

3.1 Asymmetric Electric Field Stress ……………............................................... 32

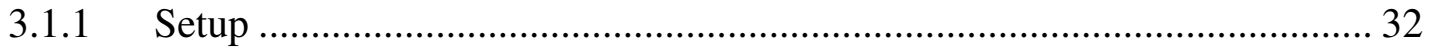

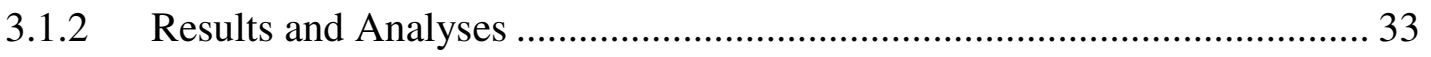




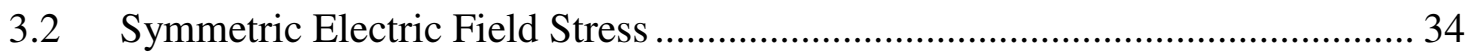

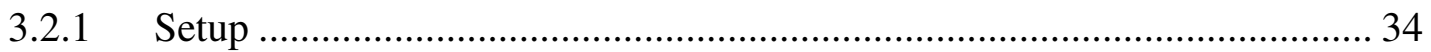

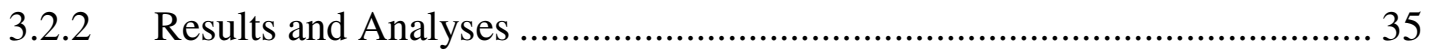

3.2.3 Device Recovery Analysis after Symmetric Electric Field Stress.............. 44

4 Experiment 2: Temperature Characterization ........................................................ 49

4.1 Effect of Temperature on General Device Performance .................................. 49

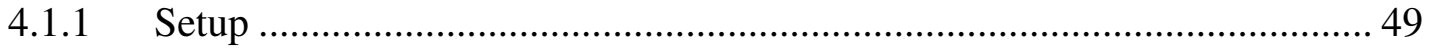

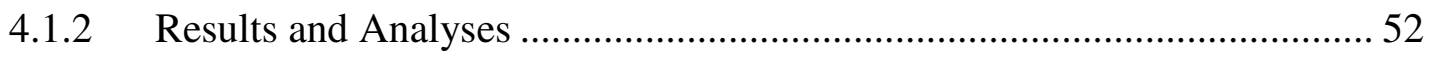

4.2 Effect of Temperature on the Critical Voltage …………….............................59

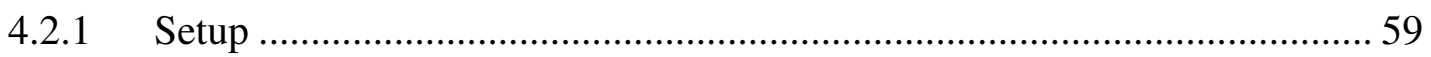

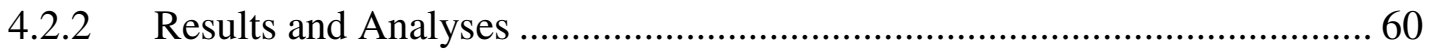

5 Experiment 3: Electron Trapping and Detrapping .................................................. 62

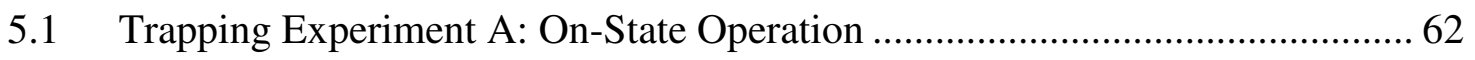

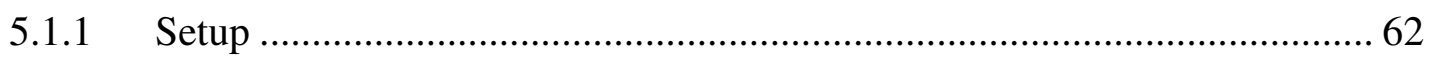

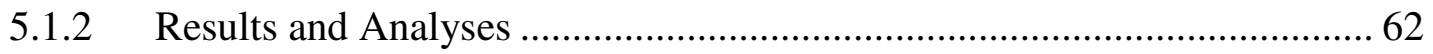

5.2 Trapping Experiment B: High Electric Field Stress ........................................ 65

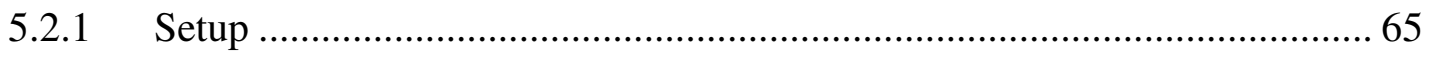

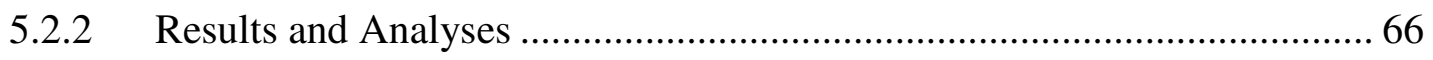

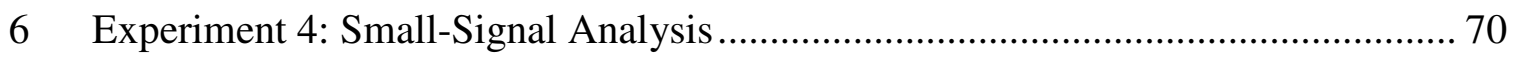

6.1 Small-Signal Model Development in Agilent ADS ……................................... 70 


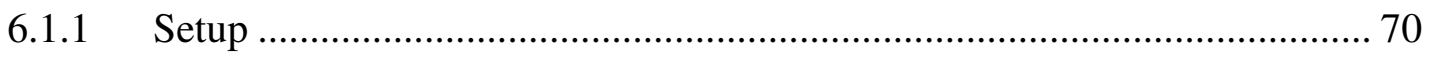

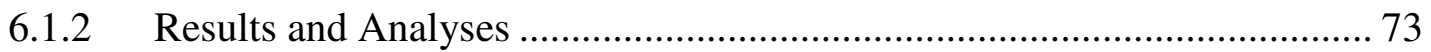

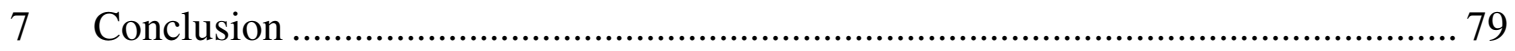

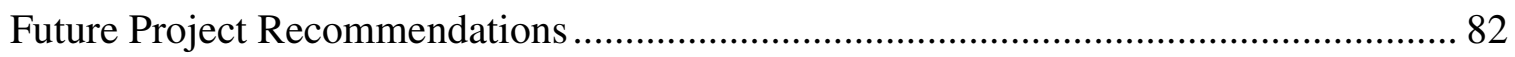

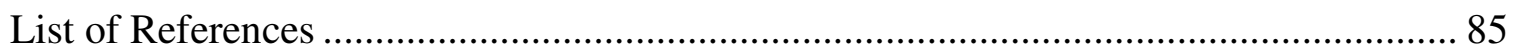

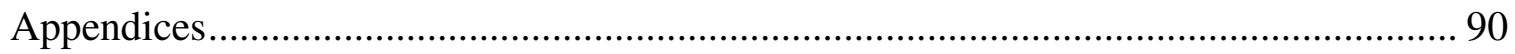

Appendix A: 1/f-Noise System Prototype …………................................................ 90

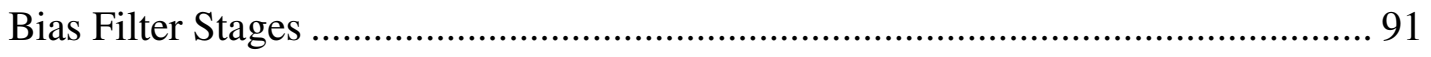

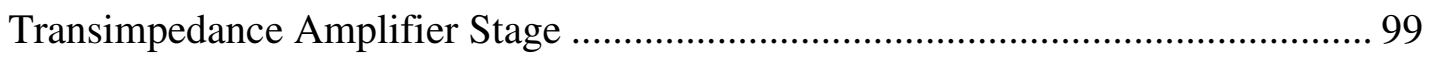

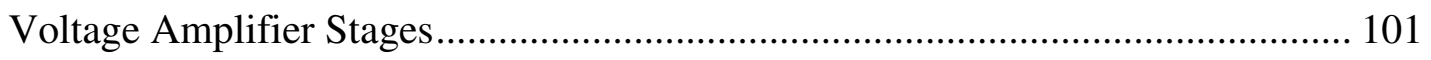

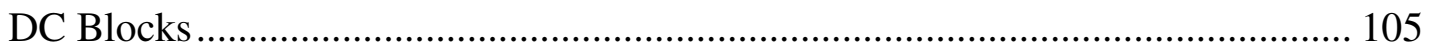

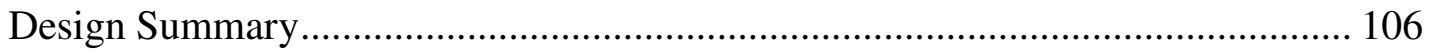

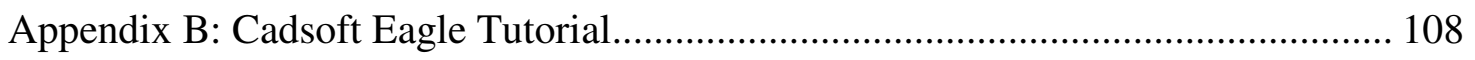

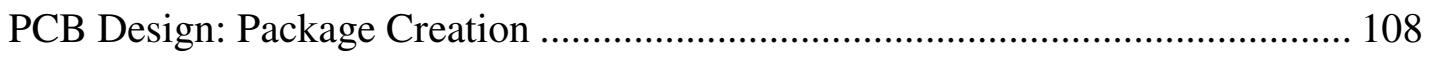

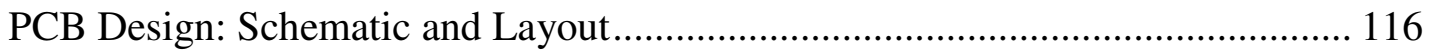

PCB Design: Gerber File Generation .................................................................. 119 


\section{LIST OF TABLES}

Table

Table 1.1 $\square$ Semiconductor materials and associated parameters [1], [7], [9]............... 6

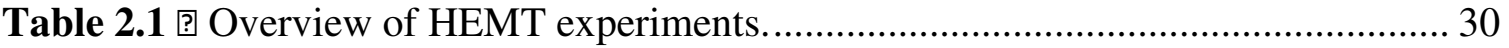

Table 2.2 ? Key instruments and software used in experiments................................. 31

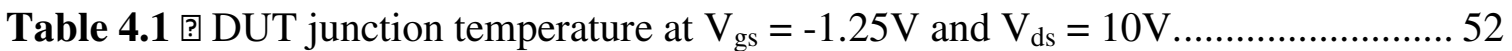

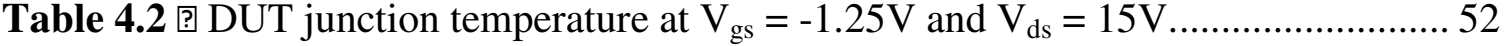

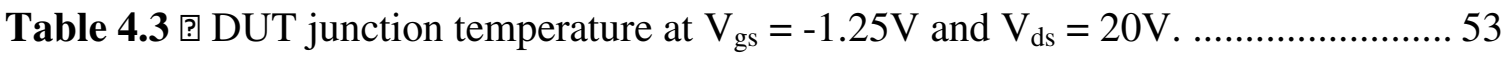

Table 6.1 [? Small-signal parameter definitions. ..................................................... 71

Table 6.2 ? Major small-signal parameter changes for pre-stress, post-stress,

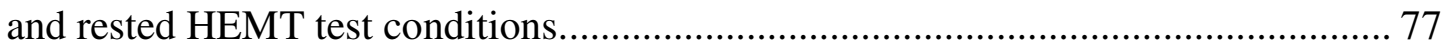

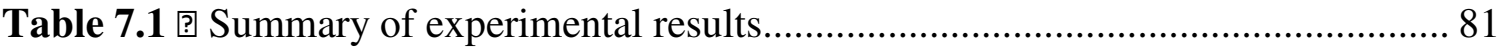

Table A.1 ? NPTB00004 DC characteristics using Toro's drain supply filter

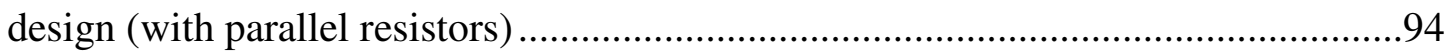

Table A.2 ? NPTB00004 DC characteristics using the $10 \Omega$ resistor in the drain filter ....96

Table A.3 ? NPTB00004 DC characteristics using the diode in the drain filter..............98

Table A.4 ? Component values for the 1/f-noise measurement system design ..............107

Table B.1 ? Necessary Gerber and drill files for board fabrication .............................122 


\section{LIST OF FIGURES}

Figure $\quad$ Page

Figure 1.1 $\square$ Performance comparison between vacuum electron devices and

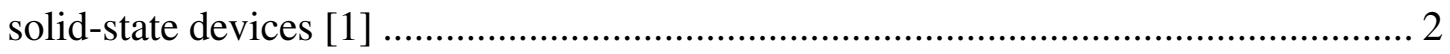

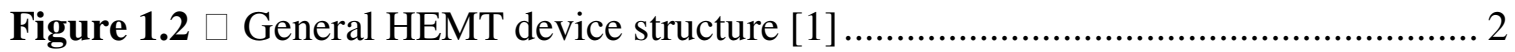

Figure 1.3 $\square$ HEMT band diagram (zero-bias gate condition) [8]............................. 4

Figure 1.4 $\square$ Direction of the vertical electric field (white arrow) and induced

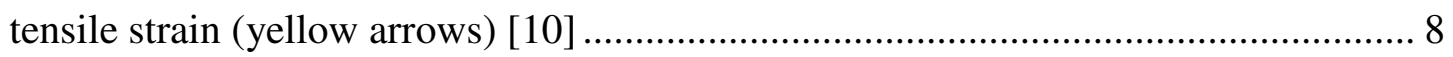

Figure 1.5 $\square$ Electron trap sites within an AlGaN/GaN HEMT [10] ........................... 8

Figure 1.6 $\square$ TEM scans showing (a) pit formation and (b) crack formation at the

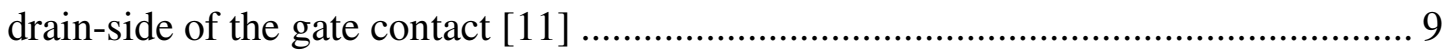

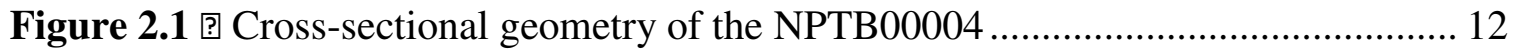

Figure 2.2 $\square$ NPTB00004 transistor die (a) layout and (b) wire-bonded into an

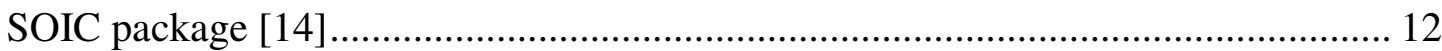

Figure 2.3 $\square$ Preliminary HEMT test board (coplanar waveguide indicated in red)...... 14

Figure 2.4 [? HEMT test structure (inset: NPTB00004 package [13]) .......................... 15

Figure 2.5 ? Heat sink attachment for a HEMT test structure .................................... 16

Figure 2.6 ? Agilent ADS schematic used for DC simulations .................................. 17

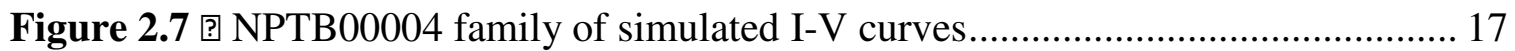

Figure 2.8 ? NPTB00004 family of simulated gate current curves ............................... 18

Figure 2.9 $\square$ NPTB00004 family of simulated transfer curves .................................. 19

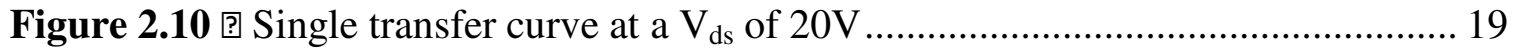

Figure 2.11 ? Agilent ADS schematic used for RF simulations ............................... 21 
Figure 2.12 $\square$ Simulated S-parameters for the NPTB00004 .................................. 21

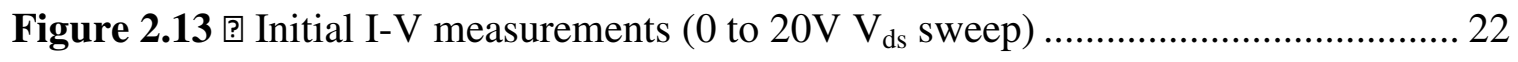

Figure 2.14 $200 \Omega$ structure used to dampen oscillations in the gate feed path ............ 23

Figure 2.15 ? Schematic representation of the $200 \Omega$ structure ................................... 23

Figure 2.16 ? Effect of time constant on system response to an excitation source ......... 24

Figure 2.17 I-V measurements using the $200 \Omega$ gate resistor and increased

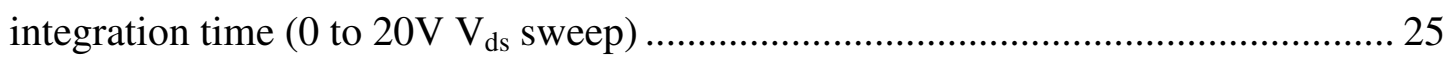

Figure 2.18 ? I-V measurements recorded by sweeping $\mathrm{V}_{\mathrm{ds}}$ from $20 \mathrm{~V}$ to $0 \mathrm{~V} \ldots \ldots \ldots \ldots \ldots . . . . . .25$

Figure 2.19 $\square$ Gate current measurements ........................................................ 26

Figure 2.20 $\square$ Measured transfer characteristics .................................................... 27

Figure 2.21 ? Bias tees configuration for S-parameter measurements ......................... 27

Figure 2.22 $\square$ S-parameter measurements........................................................ 28

Figure 3.1 ? Circuit configuration for the horizontal E-field stress experiments ........... 32

Figure 3.2 ? Gate leakage current measurement results during high asymmetric

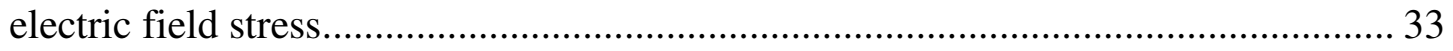

Figure 3.3 ? Gate current measurements before and after high asymmetric electric

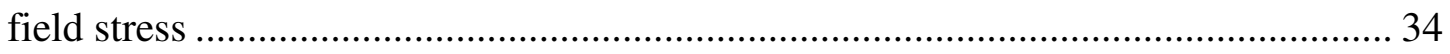

Figure 3.4 ? Circuit configuration for the high symmetric electric field stress experiments 35

Figure 3.5 ? $\mathrm{V}_{\text {crit }}$ observations using the high symmetric electric field step-stress procedure 36

Figure 3.6 ? Typical gate leakage characteristic as the gate-source voltage approaches the established $\mathrm{V}_{\text {crit }}$ 37 
Figure 3.7 ? Gate leakage current characteristic over time as $\mathrm{V}_{\mathrm{gs}}=\mathrm{V}_{\text {crit }}$ 38

Figure 3.8 ? Recorded I-V characteristics before and after high symmetric electric field step-stress procedure 38

Figure 3.9 ? Gate current measurements before and after high symmetric electric field step-stress procedure.

Figure 3.10 $\square$ Energy band diagram of a metal-semiconductor contact in thermal equilibrium [27] 41

Figure 3.11 ? Transfer characteristics before and after high symmetric electric field step-stress procedure 42

Figure 3.12 ? Measured S-parameters before and after high symmetric electric field stress 43

Figure 3.13 ? Change in I-V characteristics over a relaxation period of 6 days 45

Figure 3.14 ? Observed gate current characteristics over a relaxation period of 6 days 45

Figure 3.15 ? Observed transfer characteristics over a relaxation period of 6 days ....... 47

Figure 3.16 ? Change in S-parameters over a relaxation period of 6-days..................... 48

Figure 4.1 ? Top view of the test fixture designed for the thermal experiments 49

Figure 4.2 ? Side view concept drawing of the test fixture designed for the thermal experiments 50

Figure $4.3 \square \mathrm{I}-\mathrm{V}$ characteristics at $27^{\circ} \mathrm{C}, 50^{\circ} \mathrm{C}$, and $100^{\circ} \mathrm{C}$ 54

Figure 4.4 I-V characteristics comparison between a DUT at $27^{\circ} \mathrm{C}$ and at $27^{\circ} \mathrm{C}$ 24 hours after thermal exposure at $100^{\circ} \mathrm{C}$ 54

Figure 4.5 ? Gate current characteristics at $27^{\circ} \mathrm{C}, 50^{\circ} \mathrm{C}$, and $100^{\circ} \mathrm{C}$ 55 
Figure 4.6 ? Gate current characteristics comparison between a DUT at $27^{\circ} \mathrm{C}$ and

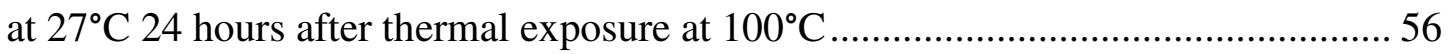

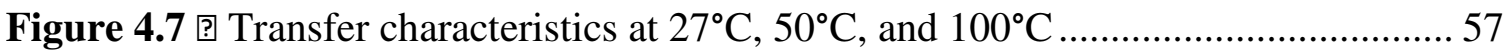

Figure 4.8 ? Transfer characteristics comparison between a DUT at $27^{\circ} \mathrm{C}$ and at

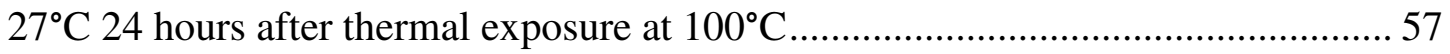

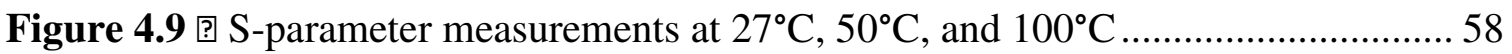

Figure 4.10 S-parameter comparison between a DUT at $27^{\circ} \mathrm{C}$ and at $27^{\circ} \mathrm{C} 24$ hours after thermal exposure at $100^{\circ} \mathrm{C}$ 59

Figure 4.11 ? Shifts in the critical voltage $\left(\mathrm{V}_{\text {crit }}\right)$ parameter with varying backplate temperatures 60

Figure 4.12 $\square$ Change in junction temperature as the gate-source voltage approaches Vcrit 61

Figure 5.1 ? Drain and gate current transients of a DUT biased in the saturation region over a 1,000 second span 63

Figure 5.2 $\square$ Detrapping experiment drain current transient results for (a) Device

$\mathrm{A}$ and $(\mathrm{b})$ Device $\mathrm{B}\left(\mathrm{V}_{\mathrm{gs}}=-1.3 \mathrm{~V}, \mathrm{~V}_{\mathrm{ds}}=2 \mathrm{~V}\right)$

Figure 5.3 $\square$ Detrapping experiment gate current transient results for (a) Device

$\mathrm{A}$ and (b) Device $\mathrm{B}\left(\mathrm{V}_{\mathrm{gs}}=-1.3 \mathrm{~V}, \mathrm{~V}_{\mathrm{ds}}=2 \mathrm{~V}\right)$

Figure 5.4 $\square$ Results of symmetric high-field stress at $\mathrm{V}_{\text {crit }}$ 66

Figure 5.5 $\square$ Drain current characteristics before and after high-field stress for (a)

Device C and (b) Device D 67

Figure 5.6 ? Gate current characteristics before and after high-field stress for (a)

Device C and (b) Device D 67 
Figure 5.7 ? Detrapping experiment drain current transient results for (a) Device

$\mathrm{C}$ and (b) Device D $\left(\mathrm{V}_{\mathrm{gs}}=-1.3 \mathrm{~V}, \mathrm{~V}_{\mathrm{ds}}=2 \mathrm{~V}\right)$

Figure 5.8 ? Detrapping experiment gate current transient results for (a) Device C

and (b) Device D $\left(\mathrm{V}_{\mathrm{gs}}=-1.3 \mathrm{~V}, \mathrm{~V}_{\mathrm{ds}}=2 \mathrm{~V}\right)$

Figure 6.1 $\square$ Small-signal model circuit with both intrinsic and extrinsic

parameters 70

Figure 6.2 $\square$ Cross-sectional geometry of the GaN-on-Si DUT with intrinsic parameters overlaid 71

Figure 6.3 ? Constructed small-signal model using Agilent ADS ............................... 73

Figure 6.4 ? Simulated S-parameters prior to fitting routine ...................................... 74

Figure 6.5 9? Agilent ADS “Tune Parameter” interface .............................................. 74

Figure 6.6 ? Simulated S-parameters after using the parameter tuning interface in

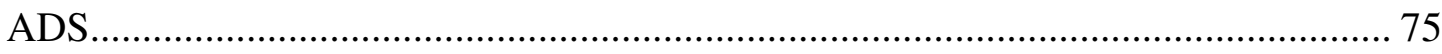

Figure 6.7 $\square$ Measured S-parameters used for small-signal modeling ......................... 76

Figure 6.8 ? Simulated S-parameters after performing a small-signal parameter fit to measured data 76

Figure A.1 $\square$ 1/f-noise measurements system for microwave transistors proposed

by Clemente Toro .90

Figure A.2 ? Gate supply filter for a FET

Figure A.3 ? Drain supply filter for a FET …...................................................... 91

Figure A.4 ? Gate supply filter for the NPTB00004 HEMT ......................................92

Figure A.5 ? Gate supply filter frequency response ..........................................92

Figure A.6 ? DC circuit simulation setup for the NPTB00004 …...............................94 
Figure A.7 [ Drain supply filter frequency response

Figure A.8 ? Q-point alteration due to resistive components in the drain supply filter....95

Figure A.9 ? Frequency response using the $10 \Omega$ resistor in the drain filter 97

Figure A.10 ? Frequency response using the diode in the drain filter .98

Figure A.11 ? Drain supply filter design

Figure A.12 ? LT6200-5 transimpedance amplifier with a sensitivity of 100V/A.........100

Figure A.13 ? LT6200-5 current to voltage conversion (100V/A sensitivity, voltage left-axis, current right-axis)

Figure A.14 ? Voltage amplifier with a gain of $14 \mathrm{~dB}(5 \mathrm{~V} / \mathrm{V})$. .101

Figure A.15 ? Observable peaking using the suggested amplifier configuration with the LT6200-5

Figure A.16 ? Simulated circuit for the LT6200-5 voltage amplifier with swept feedback capacitor values

Figure A.17 ? Simulated responses for the LT6200-5 voltage amplifier with swept feedback capacitor values .103

Figure A.18 ? ? $6200-5$ inverting amplifier configuration .103

Figure A.19 ? LT6200-5 inverting amplifier response (14dB of gain) .104

Figure A.20 ? LT6200-5 inverting amplifier cascade 104

Figure A.21 ? LT6200-5 inverting amplifier cascade response (28dB of gain) 105

Figure A.22 ? Lossless DC block 105

Figure A.23 ? DC block frequency response 106

Figure A.24 [? Final 1/f-noise measurement system design 107 
Figure B.1 ? Cadsoft Eagle main window - starting a custom library ..........................108

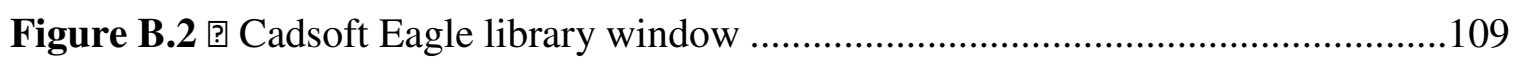

Figure B.3 ? Package creation work space with typical grid setting ..........................110

Figure B.4 ? SMD and through-hole size/dimension options, respectively ..................110

Figure B.5 ? Package design example - 8-pin DIP socket for the TL072 ....................111

Figure B.6 [ Schematic symbol creation work space ….......................................113

Figure B.7 ? Schematic symbol design example - 8-pin DIP socket for the TL072 ….114

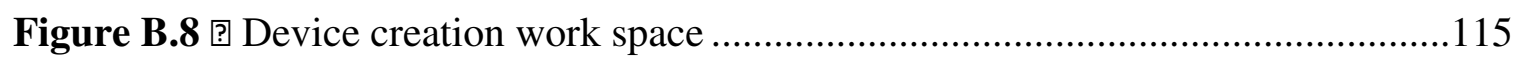

Figure B.9 ? Schematic and package symbol connection window .............................115

Figure B.10 ? Starting a new project in Eagle ...................................................116

Figure B.11 ? Starting a new schematic in the project directory ................................116

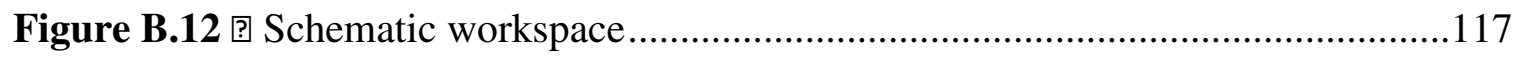

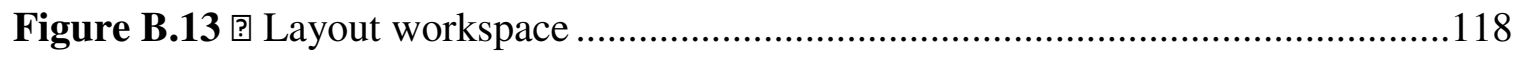

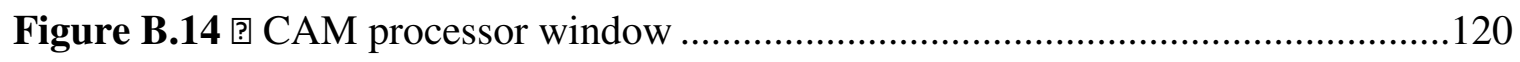

Figure B.15 ? Gerber file configuration for a 2-layer board job.................................121 


\section{Introduction}

\subsection{High Electron Mobility Transistors (HEMTs)}

Vacuum electron devices (VEDs) such as traveling-wave tubes, klystrons, and magnetrons have been leading technologies in rectifying, amplification, and switching applications for more than half a century. VEDs can be found in a range of applications such as RADAR, satellite and ground-based communication systems, and audio amplification devices. The popularity of VEDs stem from their high-speed and highpower characteristics. The trade-off for the aforementioned qualities, however, are bulkier form-factors, increased thermal dissipation, smaller bandwidths, larger power supplies, and non-instantaneous start-up operation [1].

To address the many drawbacks VEDs present, solid-state microwave transistor devices were created and are constantly being improved to match, if not exceed the exceptional capabilities of VEDs while lessening the negative trade-offs they present. Amongst the many solid-state transistors being experimented with, the most attractive candidate is the high electron mobility transistor (HEMT). Figure 1.1 shows a general comparison between a variety of VEDs and some commercially available solid-state devices such as metal semiconductor field-effect transistors (MESFETs) and pseudomorphic high electron mobility transistors (pHEMTs). While current solid-state microwave transistor technologies do not presently match VED performance, methods are being researched to increase the overall characteristics of the transistors, such as gatefinger paralleling and optimal epitaxial configurations [2]. 


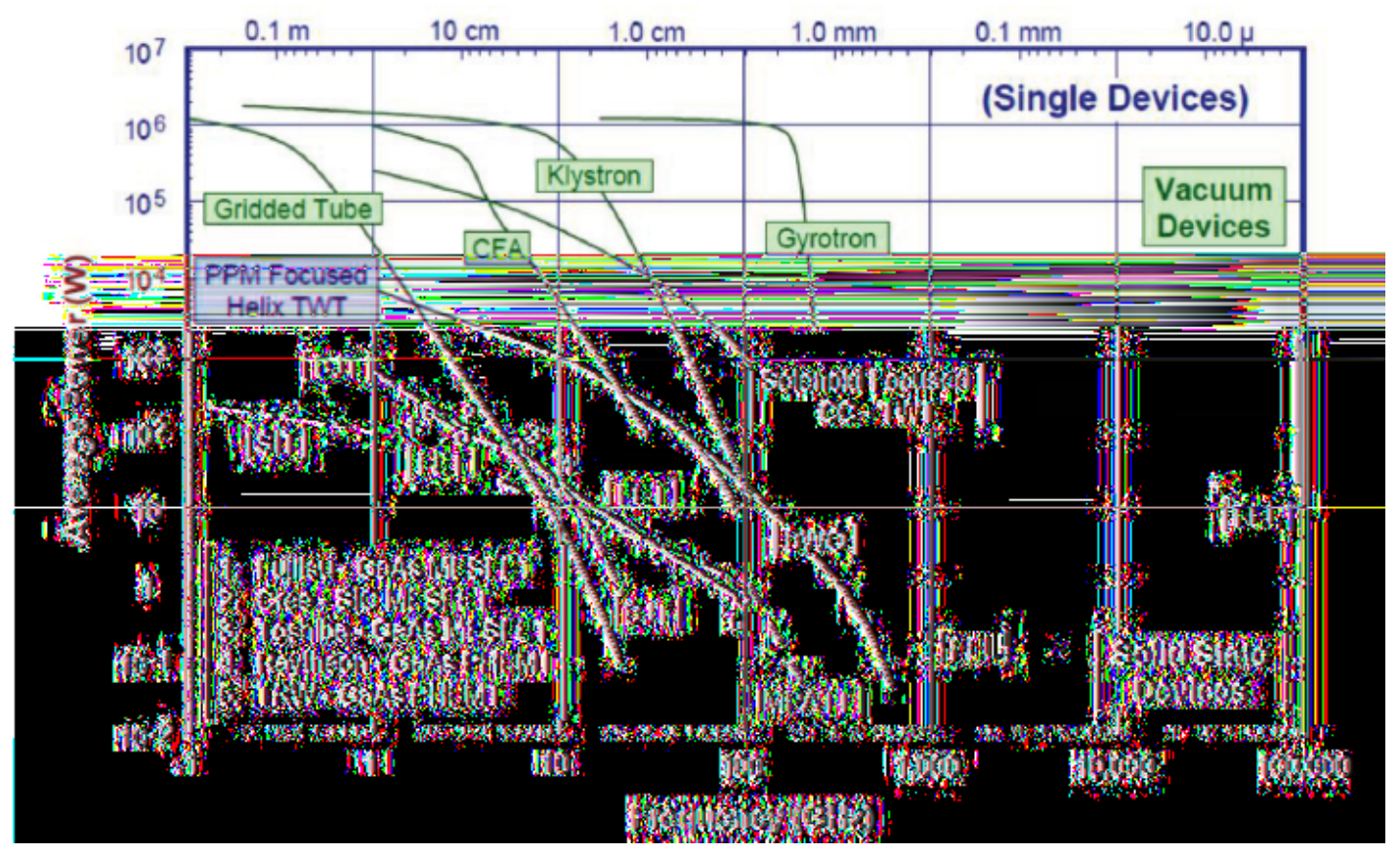

Figure 1.1 $\square$ Performance comparison between vacuum electron devices and solid-state devices [1].

HEMTs are much different from typical metal-oxide semiconductor field-effect transistors (MOSFETs) based upon the following characteristics: high electron mobility, small source resistance, high unity-gain frequency $\left(\mathrm{f}_{\mathrm{T}}\right)$, high transconductance $\left(\mathrm{g}_{\mathrm{m}}\right)$, high output resistance, and Schottky barrier integration [3]. The general structure of an HEMT is shown in Figure 1.2.

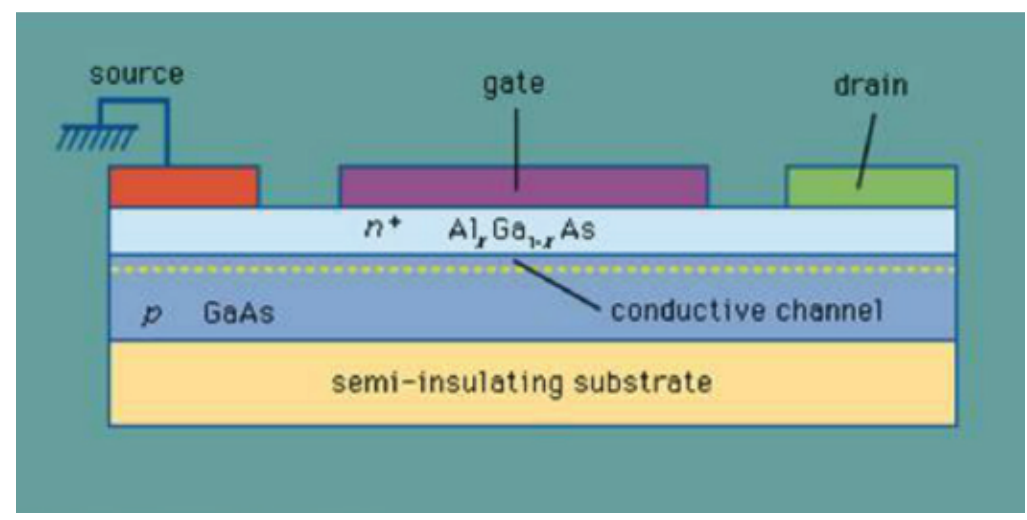

Figure 1.2 $\square$ General HEMT device structure [1]. 
The epitaxial stack-up for the devices generally consists of a semi-insulating substrate with high thermal conductivity upon which two, large bandgap energy semiconductor materials are grown. Due to the nature of the epitaxial stack, HEMTs are also classified as heterostructures. For example, in Figure 1.2, an n-type donor-supply layer of Aluminum Gallium Arsenide (AlGaAs) is grown upon an un-doped layer of Gallium Arsenide (GaAs); AlGaAs possesses a wider bandgap than GaAs due to its smaller lattice constant (atoms that form the crystal lattice in AlGaAs are much closer together) and donates free electrons to the GaAs layer due to GaAs' characteristic high electron affinity. As AlGaAs is grown upon GaAs, the lattice of the AlGaAs material becomes strained due to the lattice mismatch between the two semiconductor materials. The joining of the two semiconductor materials forms a heterojunction and an overall net piezoelectric polarization results due to the tensile strain between the two materials [1], [4]. Once the semiconductor layers are grown upon a chosen substrate, the drain, gate, and source contacts are formed, where the gate contact takes the form of a Schottky contact (metal-on-semiconductor). The benefits of using a Schottky contact over traditional gate-metal/dielectric/semiconductor configurations are reduced device capacitances (higher operating frequencies) and higher transconductances (larger power gains) [1].

A unique characteristic to HEMT devices is the development of a 2-dimensional electron gas (2DEG) at the heterointerface between the two wide bandgap semiconductor materials. A typical band diagram for an HEMT structure is shown in Figure 1.3. As seen in the diagram, band-bending takes place at the heterojuncton due to the differences in bandgap energies, which in turn forms a quantum well. The quantum well develops what 
are known as "sub-bands" for electrons to occupy (electrons tend to occupy lower energy states) [1]. The semiconductor material with a larger bandgap energy (e.g. AlGaAs) is an n-type material, providing donor. As electrons occupy the sub-bands within the quantum well, their mobility is restricted to the $\mathrm{x}$ - and $\mathrm{y}$-dimensions allowing for higher carrier density (more current, more gain) within the 2DEG of the HEMT device [5], [6], [7]. Also, because the lower bandgap semiconductor material is undoped, there is less impurity scattering in the 2DEG, leading to an increase in carrier mobility (higher speeds).

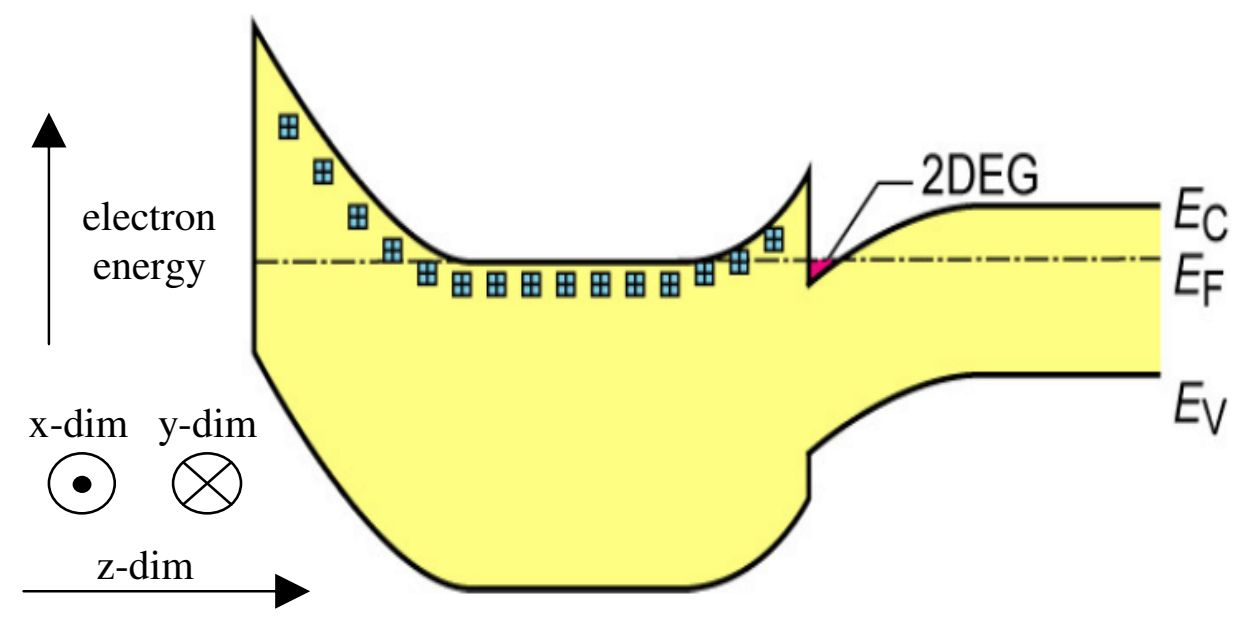

Figure 1.3 $\square$ HEMT band diagram (zero-bias gate condition) [8].

Several different semiconductor materials have been investigated for their desirable contributions to HEMT performance. Figures of merit amongst varying HEMT semiconductor materials include a large bandgap energy, a low dielectric constant, a high thermal conductance, and a high critical breakdown field. Table 1.1 lists popular semiconductor materials used in HEMT fabrication. The previously mentioned parameters are desirable for the following reasons: 
- Large Bandgap Energy $\left(\mathrm{E}_{\mathrm{g}}\right.$ ): Determines the transistors ability to operate under high levels of temperature, radiation, and electric fields [1], [7].

- Low Dielectric Constant $\left(\varepsilon_{\mathrm{r}}\right)$ : Reduces the overall capacitive area within the device, which in turn increases the unity-gain frequency $\left(\mathrm{f}_{\mathrm{T}}\right)$ and maximum frequency of oscillation ( $f_{\max }$, where the unilateral power gain equals unity) of the transistor. Referring to Equations (1.1), (1.2), and (1.3), if the dielectric constant increases, both $\mathrm{f}_{\mathrm{T}}$ and $\mathrm{f}_{\max }$ will decrease proportionally [1].

$$
\begin{gathered}
C \propto \varepsilon_{r} A \\
f_{T}=\frac{g_{m}}{2 \pi C_{g s}} \\
f_{\max }=\frac{f_{T}}{2} \sqrt{\frac{R_{d s}}{R_{g}}}
\end{gathered}
$$

- High Thermal Conductance (K): Under high-power operation, if a device cannot properly dissipate heat due to self-heating, the device can fail as a result.

- High Critical Breakdown Field $\left(\mathrm{E}_{\mathrm{C}}\right)$ : Leads to higher realizable breakdown voltages that can provide large power densities when coupled with high current densities within the 2DEG of the HEMT [7]. 
Table 1.1 $\square$ Semiconductor materials and associated parameters [1], [7], [9].

\begin{tabular}{|c|c|c|c|c|}
\hline & \multicolumn{4}{|c|}{ Material Parameters } \\
\hline Material & $E_{g}(e V)$ & $\varepsilon_{r}$ & $K\left(W /{ }^{\circ} \mathrm{K}-\mathrm{cm}\right)$ & $E_{c}(V / m)$ \\
\hline Vacuum & - & 1 & - & - \\
\hline $\mathrm{Si}$ & 1.12 & 11.9 & 1.5 & $3 \times 10^{5}$ \\
\hline GaAs & 1.43 & 12.5 & 0.54 & $4 \times 10^{5}$ \\
\hline InP & 1.34 & 12.4 & 0.67 & $4.5 \times 10^{5}$ \\
\hline 3C-SiC & 2.3 & 9.7 & 4 & $1.8 \times 10^{6}$ \\
\hline $4 \mathrm{H}-\mathrm{SiC}$ & 3.2 & 10.0 & 4 & $3.5 \times 10^{6}$ \\
\hline $6 \mathrm{H}-\mathrm{SiC}$ & 2.86 & 10.0 & 4 & $3.8 \times 10^{6}$ \\
\hline $\mathrm{GaN}$ & 3.4 & 9.5 & 1.3 & $3.3 \times 10^{6}$ \\
\hline Diamond & 5.6 & 5.3 & $20-30$ & $5 \times 10^{6}$ \\
\hline Sapphire & 10 & $7.5-11.5$ & $35-40$ & $(15$ to 50$) \times 10^{6}$ \\
\hline
\end{tabular}

\subsection{Gallium Nitride (GaN) HEMTs}

Out of the available semiconductor materials, $\mathrm{GaN}$ is rapidly becoming a prime candidate in the fabrication of HEMTs. Based upon the parameters from Table 1.1, GaN possesses an exceptional bandgap energy (3.4eV), dielectric constant (9.5), and critical breakdown field $(3.3 \mathrm{MV} / \mathrm{m})$ in comparison to GaAs and Indium Phosphide (InP) materials (GaAs and InP being competing materials). While the thermal conductivity of $\mathrm{GaN}$ is relatively poor in comparison to other semiconductor materials $\left(1.3 \mathrm{~W} /{ }^{\circ} \mathrm{K}-\mathrm{cm}\right)$, other materials such as Silicon Carbide (SiC), Silicon (Si), Diamond, and Sapphire can be used as a thermally conductive substrate for the GaN HEMT.

A question then arises as to why GaN-based HEMTs are not as widely accepted in the commercial and military markets. The two primary concerns revolving the technology are reliability and reproducibility. Because GaN technology has not fully matured, many different process variations exist for the device. For example, a variety of epitaxial stack- 
ups exist, which in turn affects several of the HEMT's device characteristics such as I-V parameters, electric field distribution, power efficiency, and so forth. GaN HEMT substrates are also inconsistent between manufacturers; some may choose $\mathrm{SiC}$ due to its high thermal conductivity and higher lattice matching, while others may choose Si due to its cost-effectiveness. Performance variation amongst a group of HEMTs fabricated with the same epitaxial stack and substrate may also exist.

One of the major reliability concerns in current $\mathrm{GaN}$ technologies is the creation of crystallographic defect formations during fabrication and through the inverse piezoelectric effect [10]. The inverse piezoelectric effect occurs when a high vertical electric field perpendicular to the device's substrate is present across both semiconductor materials, which in turn promotes tensile strain (Figure 1.4). From [10], the lattice dislocations and defect formations were determined to have formed under a specific gate voltage bias known as the critical voltage $\left(\mathrm{V}_{\text {crit }}\right)$. The critical voltage is defined as the bias voltage at which a change in device performance is observed, which in turn is attributed to the sudden formation of defects within the device. The problem surrounding defect formations are their ability to facilitate electron trap states, with varying trap densities scattered amongst the sites of dislocation. Figure 1.5 shows an example of existing electron trap sites within the AlGaN layer of a GaN HEMT. Electron trapping ultimately degrades the DC performance of the device, more specifically by causing current reduction due to depletion of the 2DEG and increased gate leakage though the Schottky barrier [1], [10]. 


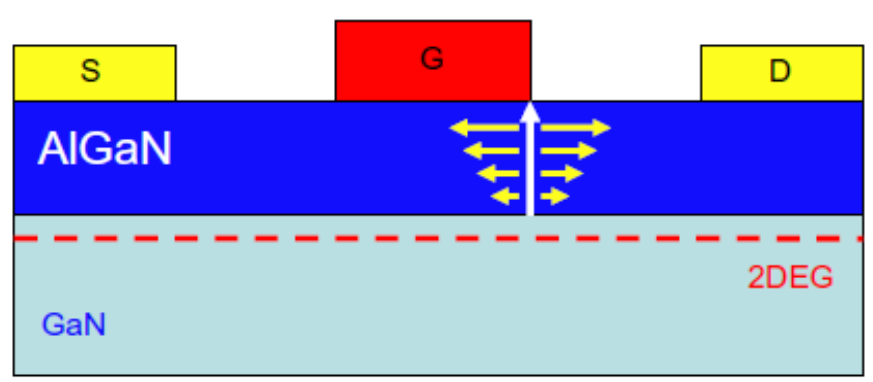

Figure 1.4 $\square$ Direction of the vertical electric field (white arrow) and induced tensile strain (yellow arrows) [10].

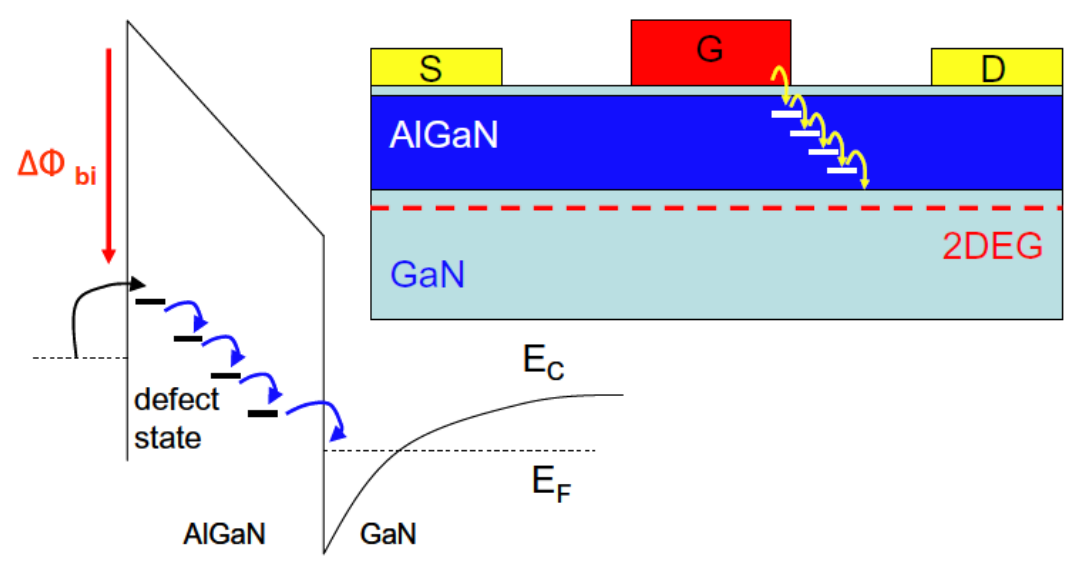

Figure 1.5 $\square$ Electron trap sites within an AlGaN/GaN HEMT [10].

Other research has found the existence of pit and crack formations under the drain-side edge of the gate contact after being exposed to high electric field stresses [11]. The pit formations that were observed through electrical degradation caused a worst-case $70 \%$ drop in drain current and a $0.6 \mathrm{~V}$ positive shift in threshold voltage. Crack formations that were observed signified the onset of abrupt device failure. Figure 1.6 shows the resulting pit and crack formations seen in [11] through the use of transmission electron microscope (TEM) scans. 


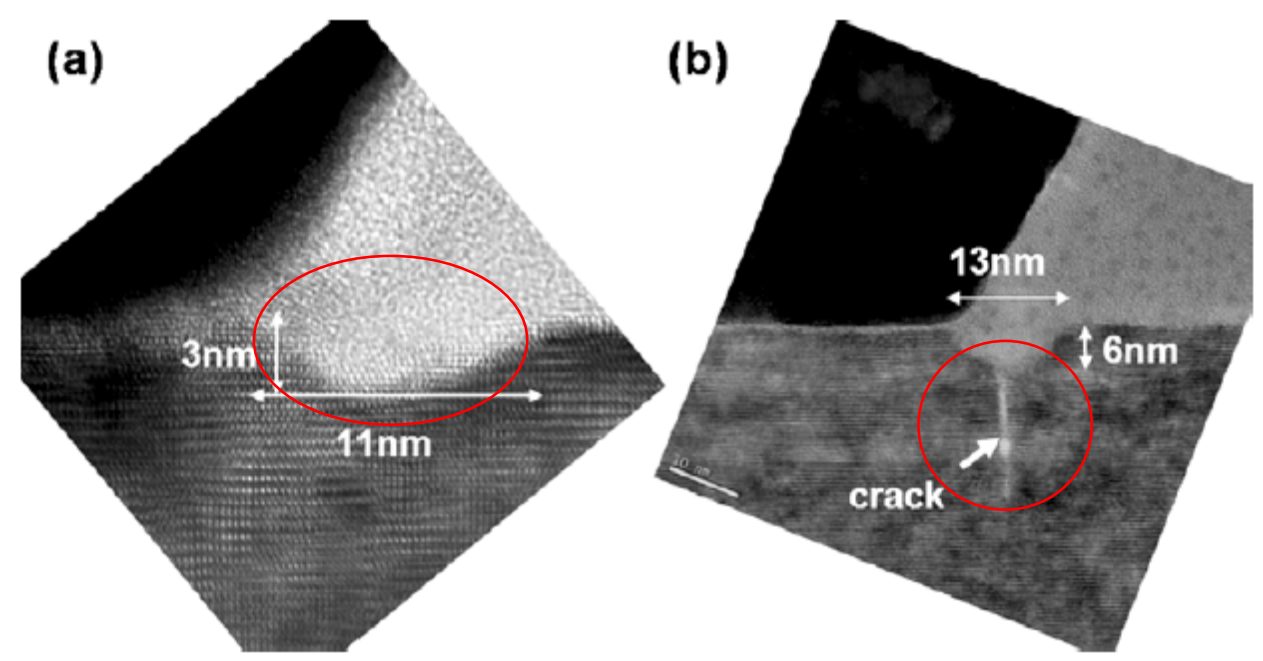

Figure 1.6 $\square$ TEM scans showing (a) pit formation and (b) crack formation at the drain-side of the gate contact [11].

\subsection{Research Focus}

The work presented in this paper utilizes the high-field experiment and accelerated lifetime experiment methodologies of [10] and [11] to investigate degradation mechanisms within GaN-on-Si HEMTs (GaN grown upon Si-substrates). GaN-on-Si technology has become attractive due to the low-cost of fabrication associated with using a Si-substrate, thus making research efforts on the technology more desirable. Other advantages to Si-substrate usage are ease of wafer thinning, via-hole formation, and $\mathrm{AuSi}$ eutectic die attach [12]. Because a 17\% lattice mismatch and 56\% thermal expansion coefficient mismatch exists between $\mathrm{GaN}$ and $\mathrm{Si}$, utilizing $\mathrm{GaN}$-on-Si devices in accelerated lifetime experiments may be more beneficial for observing sudden degradation events.

The main purpose of the experiments are to determine how the DC-, RF-, and thermal-level characteristics of the target HEMTs degrade under high-field stress, and what the underlying mechanisms are leading to the observed modes of degradation. The 
research presented in this paper is an on-going collaboration between Cal Poly, San Luis Obispo and the Naval Postgraduate School. The collaborative work between the two schools involved performing experimental analyses, extracting "black box" results, and then identifying the mechanisms associated with the degradation results at the material level. 


\section{Device Under Test (DUT) Overview and Test Plan}

\subsection{Selected DUT for Experiments}

The NPTB00004 from Nitronex was selected as the representative GaN-on-Si device to be evaluated. A single NPTB00004 SOIC package is capable of producing 5W of continuous wave $(\mathrm{CW})$ power and operates from $\mathrm{DC}$ up to $6 \mathrm{GHz}$ [13]. Device specifications and the cross-sectional geometry for the GaN-on-Si HEMT are listed below and shown in Figure 2.1, respectively, and are derived from [14], [15], [16], and [17]. A physical picture of the NPTB00004 die is shown in Figure 2.2.

- $\quad 0.5 \mu \mathrm{m}$ (length) Ni/Au (Schottky) T-gate electrodes

- $2 \mathrm{~mm}$ gate periphery

- $\quad 1 \mu \mathrm{m}$ gate-source spacing and $3 \mu \mathrm{m}$ gate-drain spacing

- $\mathrm{Ti} / \mathrm{Al} / \mathrm{Ni} / \mathrm{Au}$ ohmic metallization

- $490 \mathrm{~nm} \mathrm{SiN}_{\mathrm{X}}$ passivation layer and $\mathrm{SiN}_{X}$ encapsulation layer

- $\quad 1.5 n m$ GaN cap layer

- $18 \mathrm{~nm} \mathrm{Al}_{0.26} \mathrm{GaN}_{0.74}$ barrier and $800 \mathrm{~nm}$ GaN barrier

- $\mathrm{AlGaN}$ transition layer and AlN nucleation layer

- Electroplated gold air-bridge interconnect level

- Source-connected field plate (SCFP)

- $\quad 150 \mu \mathrm{m}$ Si-substrate $(10,000 \Omega-\mathrm{cm})$ 


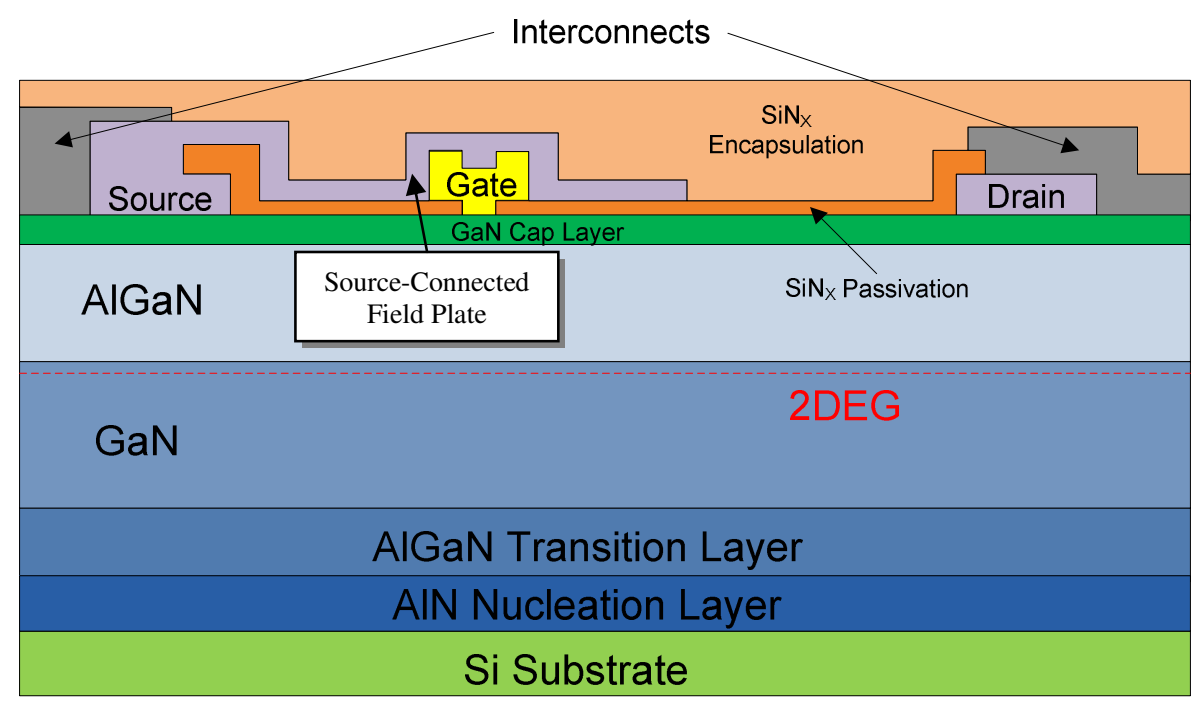

Figure 2.1 [ Cross-sectional geometry of the NPTB00004.

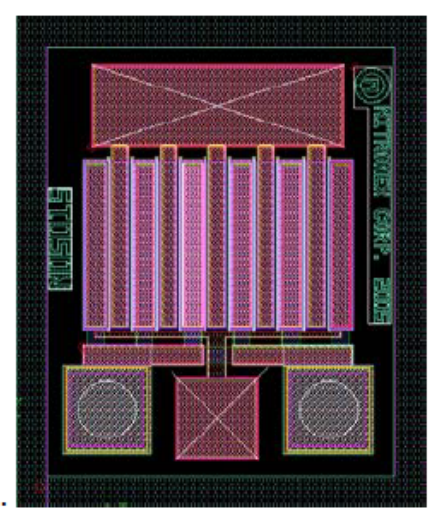

(a)
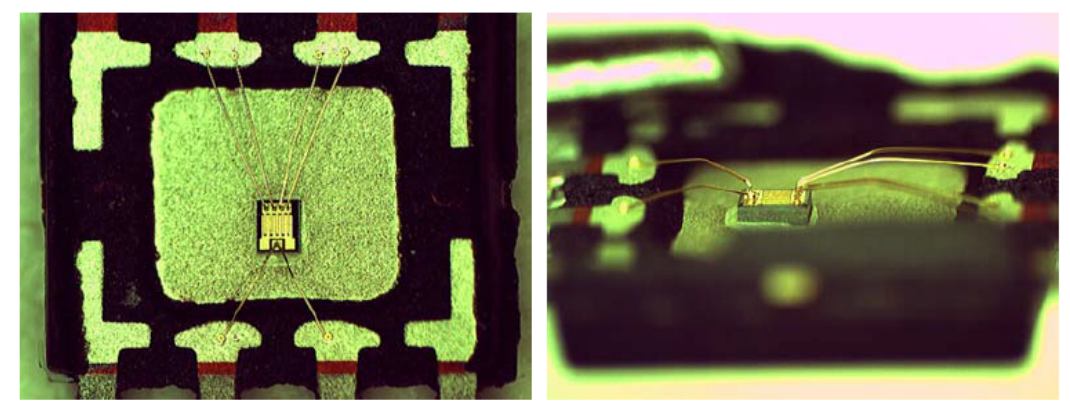

(b)

Figure 2.2 $\square$ NPTB00004 transistor die (a) layout and (b) wire-bonded into an SOIC package [14]. 
As described in [12], [15], [16], and [17] the NPTB00004 was developed using metal-organic chemical vapor deposition (MOCVD). Before the primary semiconductor barriers were grown, an AlN nucleation layer was added to prevent unwanted Ga-Si interactions and an $\mathrm{AlGaN}$ transition layer was included to provide an increased thermal and lattice match between the GaN layer and Si substrate. Each GaN-on-Si die can be paralleled with multiple dies using the interconnects to form an HEMT with a higher power density. The NPTB00004, however, only utilizes a single die.

One key feature of the GaN-on-Si HEMT that improves performance is the source-connected field plate (SCFP). Reliability studies have demonstrated how inducing the inverse piezoelectric effect in the $\mathrm{AlGaN}$ barrier facilitates crystallographic defect formation under the drain-side of the gate contact [18], [19]. By including an SCFP in the growth process, the peak vertical electric field under the gate is redistributed, allowing HEMTs to achieve higher breakdown voltages, lower gate leakage currents, higher cutoff frequencies, higher maximum available gains, and lower gate-drain capacitances [7], $[15]$.

Another important feature within the epitaxial stack is the inclusion of a GaN cap layer. The GaN cap serves two purposes. The first key feature is how the GaN cap mitigates the high vertical electric field seen at the drain-side edge of the gate contact when biased in the off-state. Reduction of the vertical electric field was observed to have reduced the amount of current collapse and gate leakage current experienced within the GaN HEMTs [20]. The second key feature improves the overall surface morphology of the epitaxial stack. Researchers in [20] observed a large increase in their devices' 
breakdown voltage ( $100 \mathrm{~V}$ to $350 \mathrm{~V})$ due to the ease of forming the gate electrodes on a much smoother surface, as opposed to the cruder surface of an $\mathrm{AlGaN}$ cap layer.

Before experimenting with the NPTB00004, a standard test structure needed to be created for each DUT to ensure relative consistency between measurements. The preliminary board that was considered was a basic PCB fabricated on FR4 and constructed in a $50 \Omega$ coplanar waveguide configuration (Figure 2.3). The board was created using Cadsoft Eagle schematic and layout software (an Eagle tutorial can be found in Appendix B: Cadsoft Eagle Tutorial). The PCB design was ultimately discarded due to high lead-times and cost-effectiveness. Because the study involved testing a large quantity of NPTB00004 HEMTs, a different method needed to be used in order to speed up structure creation at a low-lost.

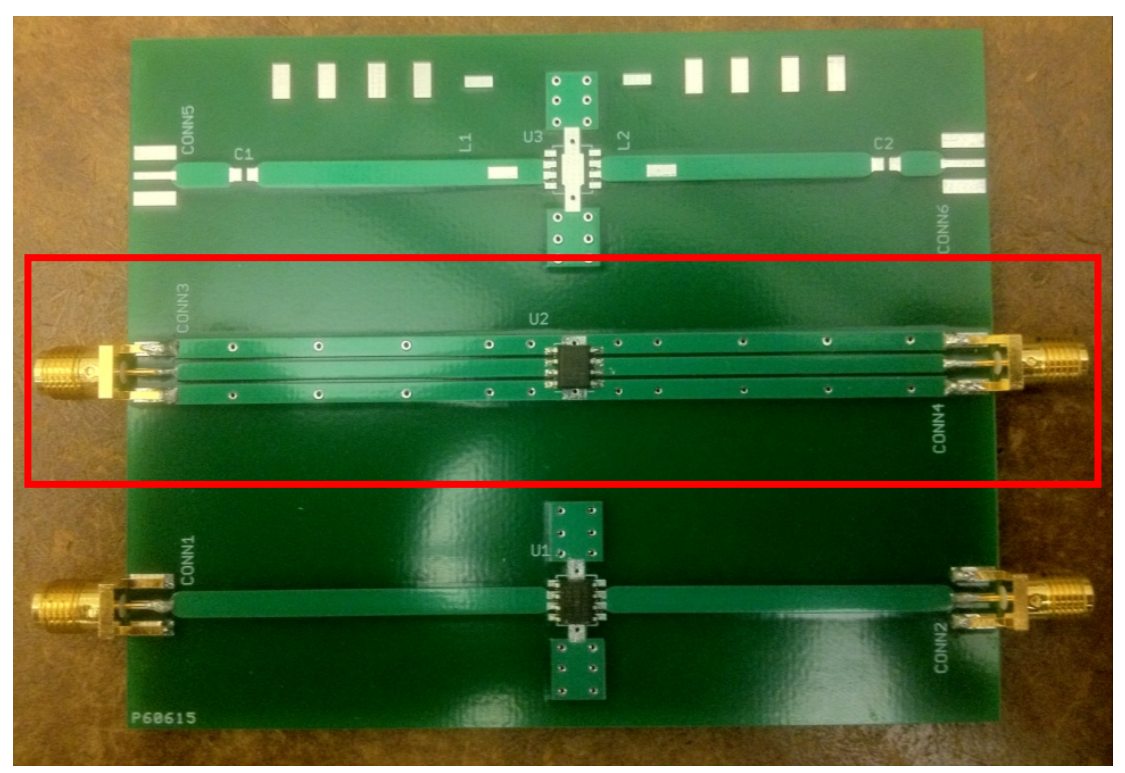

Figure 2.3 $\square$ Preliminary HEMT test board (coplanar waveguide indicated in red).

A simple copper DUT structure, shown in Figure 2.4, was used as the test structure of choice. Each structure utilizes a 40mm x 55mm double-sided copper FR4 board, which serves as a grounding plane for the DUT. The source-pad of the HEMT 
(located on the underside of the SOIC package) was then soldered to the copper using

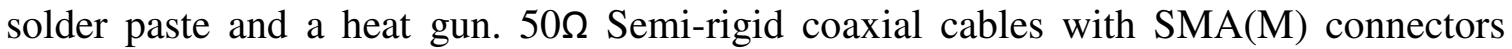
were used to provide electrical signals to the device. The coaxial line lengths varied from device to device, which did not affect device characterization during DC-level experiments. During RF experiments, however, the recorded lengths were used to reference-plane shift S-parameter measurements up to the device's package leads. To prevent parasitic inductances along the transmission lines, the outer conductors of each coaxial line were soldered to the copper ground plane at the edge of the copper board and near the leads of the DUT. Because the NPTB00004 possesses the ability to provide up to $5 \mathrm{~W}$ of power, a $40 \mathrm{~mm} \times 55 \mathrm{~mm}(6.5 \mathrm{~mm}$ thickness $)$ heat sink was added to each structure to provide proper dissipation of heat. A single heat sink attachment is shown in Figure 2.5.

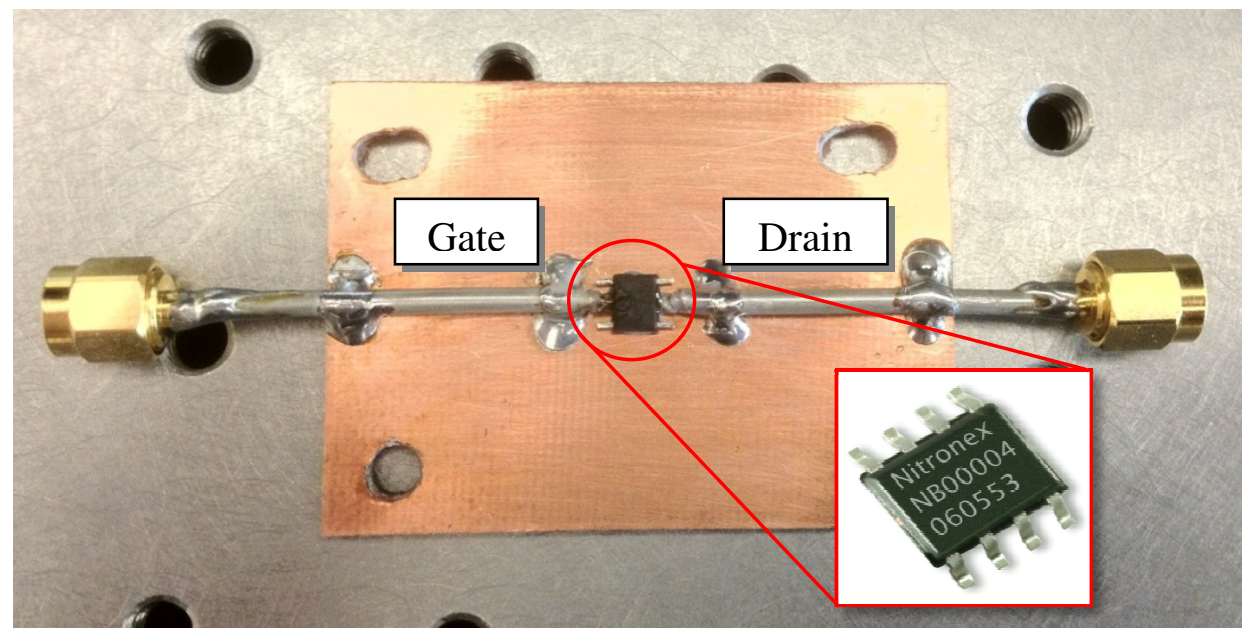

Figure 2.4 ? HEMT test structure (inset: NPTB00004 package [13]). 


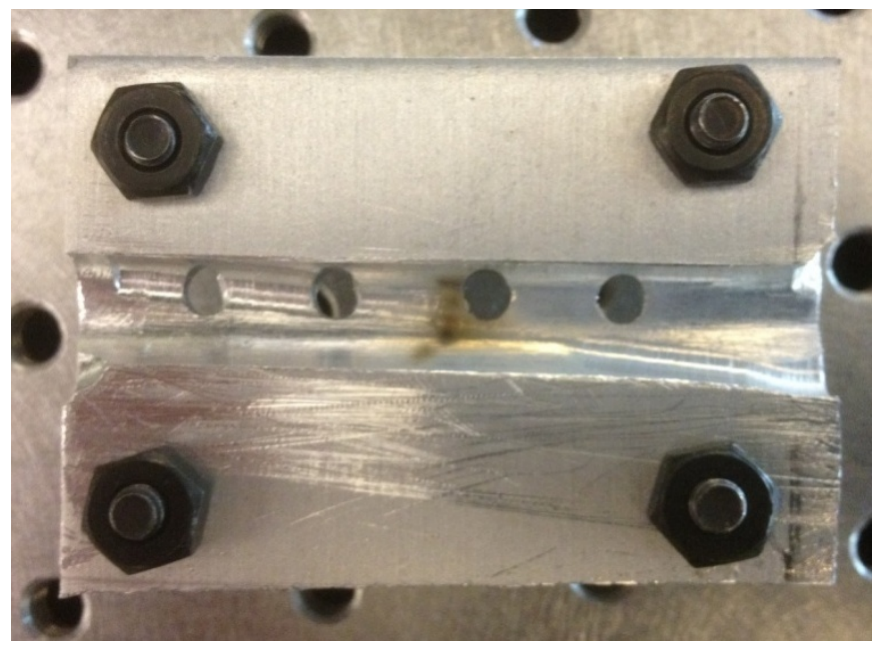

Figure 2.5 ? Heat sink attachment for a HEMT test structure.

\subsection{Simulation of the HEMT's DC and RF Characteristics}

According to [13], the NPTB00004 can achieve a maximum drain-source voltage $\left(\mathrm{V}_{\mathrm{ds}}\right)$ of $28 \mathrm{~V}$, possesses a typical threshold voltage $\left(\mathrm{V}_{\mathrm{t}}\right)$ of $-1.5 \mathrm{~V}$, and utilizes a quiescent gate voltage $\left(\mathrm{V}_{\mathrm{gsq}}\right)$ of $-1.3 \mathrm{~V}$. At a $\mathrm{V}_{\mathrm{gsq}}$ of $-1.3 \mathrm{~V}$ and $\mathrm{V}_{\mathrm{ds}}$ of $28 \mathrm{~V}$, a drain current $\left(\mathrm{I}_{\mathrm{d}}\right)$ of $50 \mathrm{~mA}$ is produced, which in turn allows for $1.4 \mathrm{~W}$ of power dissipation. Due to power limitations for the instruments used in the laboratory, the DUTs are only evaluated for $\mathrm{V}_{\mathrm{gs}} \leq-1.25 \mathrm{~V}$ and $\mathrm{V}_{\mathrm{ds}} \leq 20 \mathrm{~V}$. All simulations for the DUT were performed using Agilent Advanced Design System (ADS). All simulations assumed a device temperature of $27^{\circ} \mathrm{C}$.

\subsubsection{I-V Characteristics}

Figure 2.6 shows the simulation schematic used to analyze the NPTB00004's I-V, gate current, and transfer characteristics. The list below Figure 2.6 shows the DC conditions used during each simulation. Figure 2.7 and Figure 2.8 are the simulated I-V and gate current characteristics, respectively. $\mathrm{A} \mathrm{V}_{\mathrm{gsq}}$ of $-1.3 \mathrm{~V}$ and a $\mathrm{V}_{\mathrm{ds}}$ of $20 \mathrm{~V}$ were chosen as the standard biases for all experiments performed on the NPTB00004. At the aforementioned Q-point, an $\mathrm{I}_{\mathrm{dsq}}$ of approximately $60 \mathrm{~mA}$ was simulated, resulting in $1.2 \mathrm{~W}$ 
of dissipated power. Power at the gate terminal was simulated to be negligible from the corresponding gate current measurement in Figure 2.8.

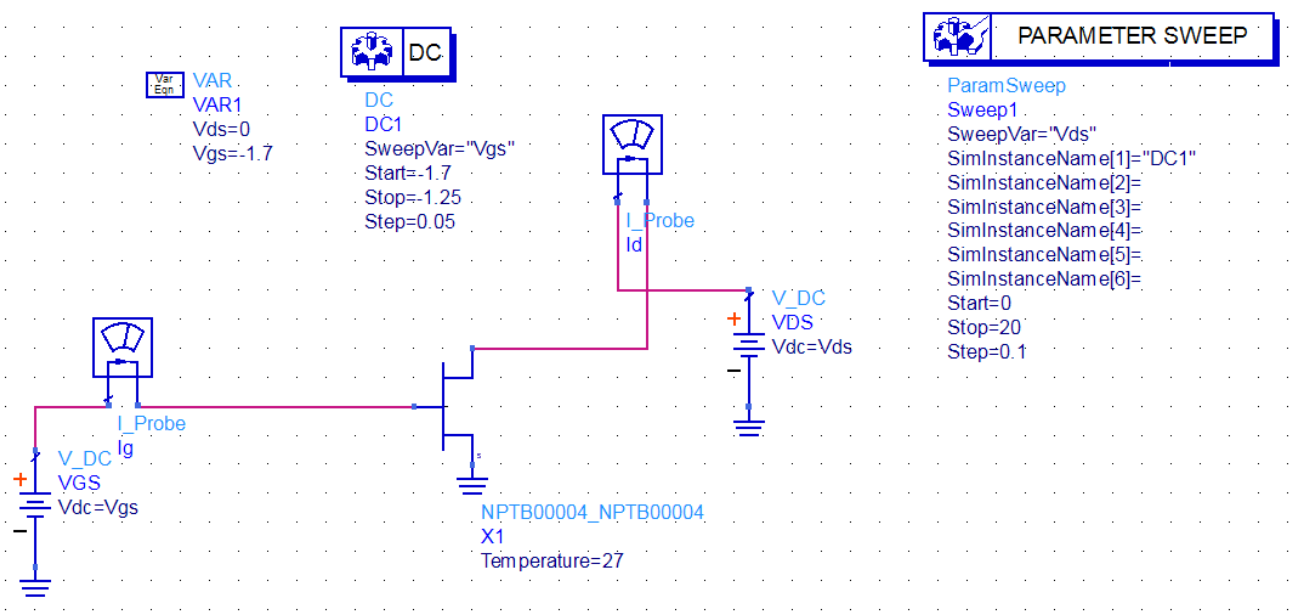

Figure 2.6 ? Agilent ADS schematic used for DC simulations.

- I-V and gate current characteristics: bias conditions

$\circ \mathrm{V}_{\mathrm{ds}}=0$ to $20 \mathrm{~V}(100 \mathrm{mV}$ increments $)$

$\circ \mathrm{V}_{\mathrm{gs}}=-1.7 \mathrm{~V}$ to $-1.25 \mathrm{~V}(50 \mathrm{mV}$ increments $)$

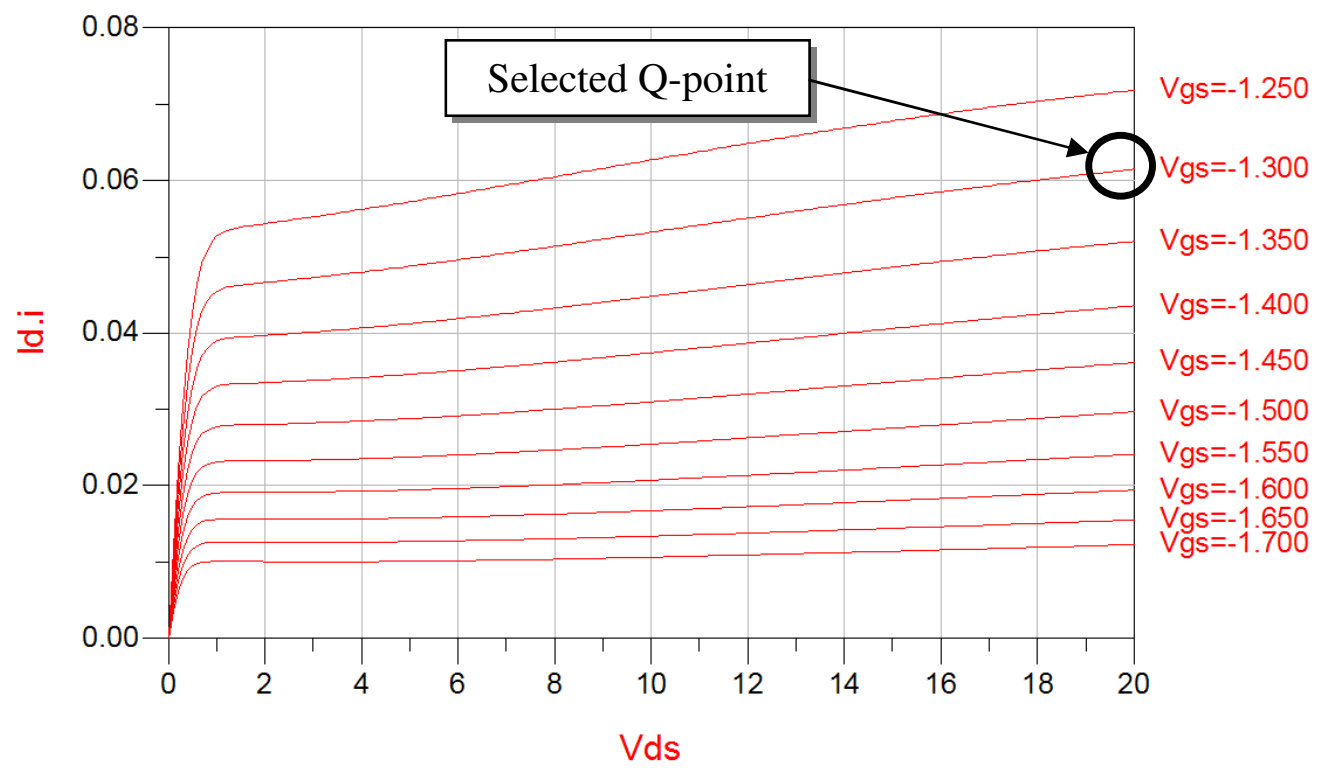

Figure 2.7 ? NPTB00004 family of simulated I-V curves. 


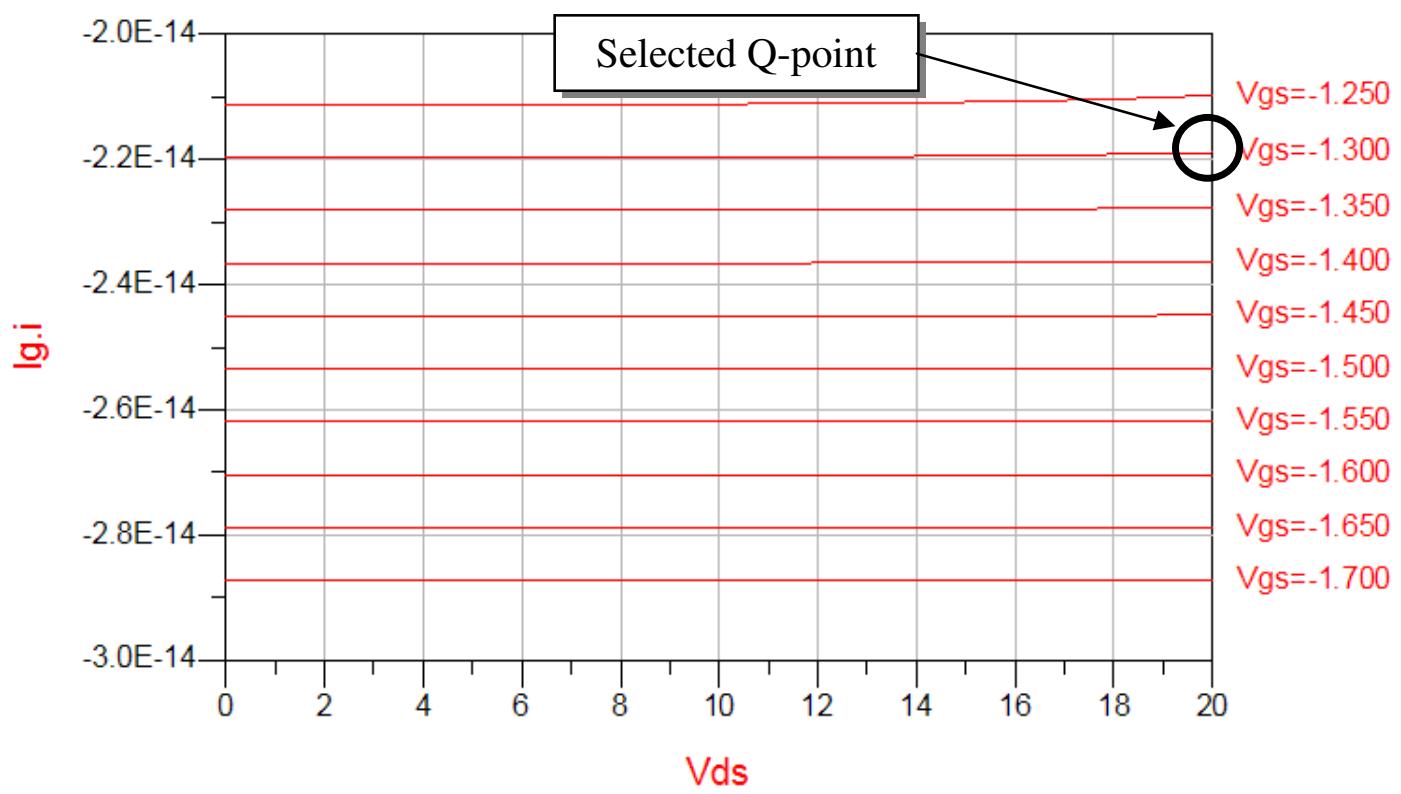

Figure 2.8 ? NPTB00004 family of simulated gate current curves

\subsubsection{Transfer Characteristics}

The following is a list of DC conditions used to simulate the transfer characteristics for the DUT. Figure 2.9 shows a family of transfer curves generated from the bias conditions in the list. A second simulation was done to evaluate the transfer characteristics at a $\mathrm{V}_{\mathrm{ds}}$ of $20 \mathrm{~V}$, which was used in as a baseline for proceeding experiments. The results are shown in Figure 2.10.

- Transfer characteristics: bias conditions

$$
\begin{aligned}
& \circ \mathrm{V}_{\mathrm{ds}}=0 \text { to } 20 \mathrm{~V}(100 \mathrm{mV} \text { increments }) \\
& \circ \mathrm{V}_{\mathrm{gs}}=-2 \mathrm{~V} \text { to }-1.25 \mathrm{~V}(50 \mathrm{mV} \text { increments })
\end{aligned}
$$




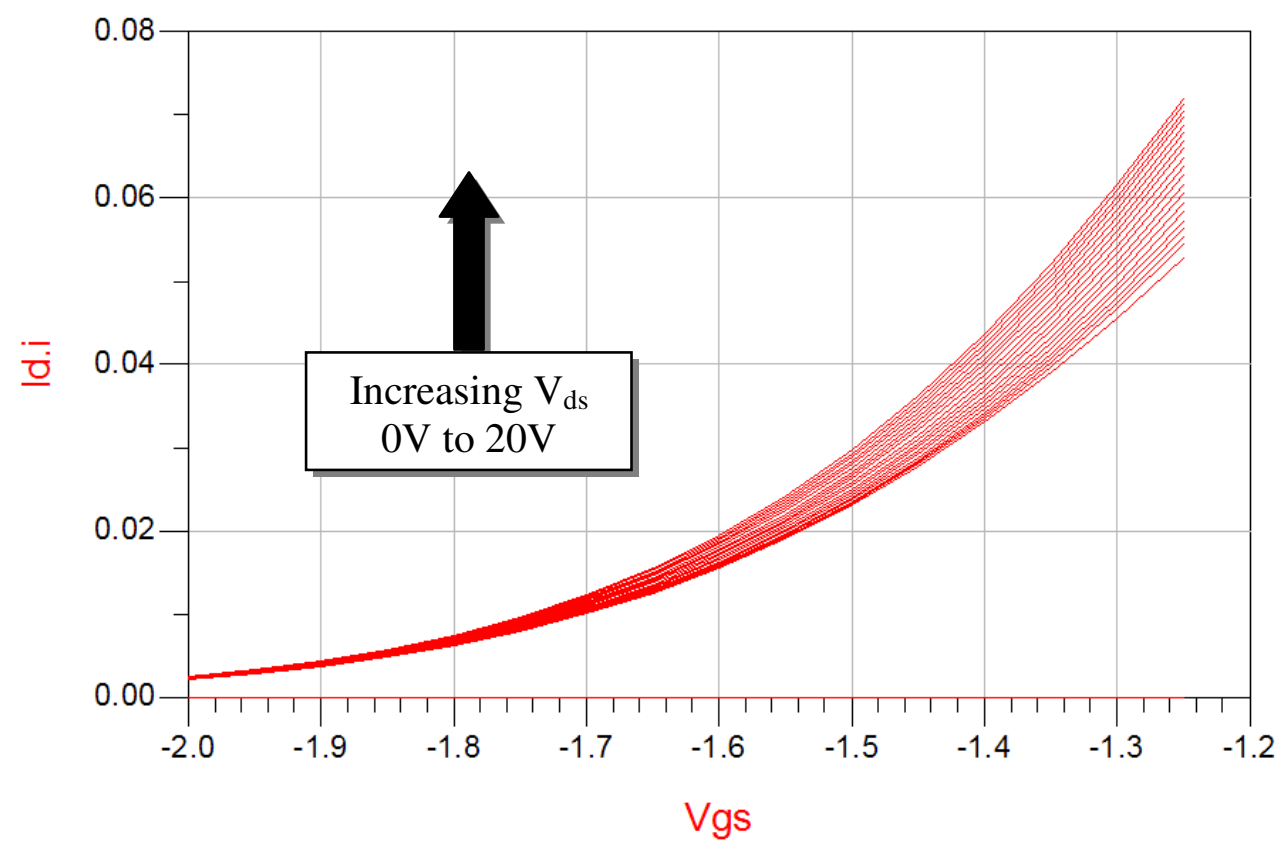

Figure 2.9 $\square$ NPTB00004 family of simulated transfer curves.

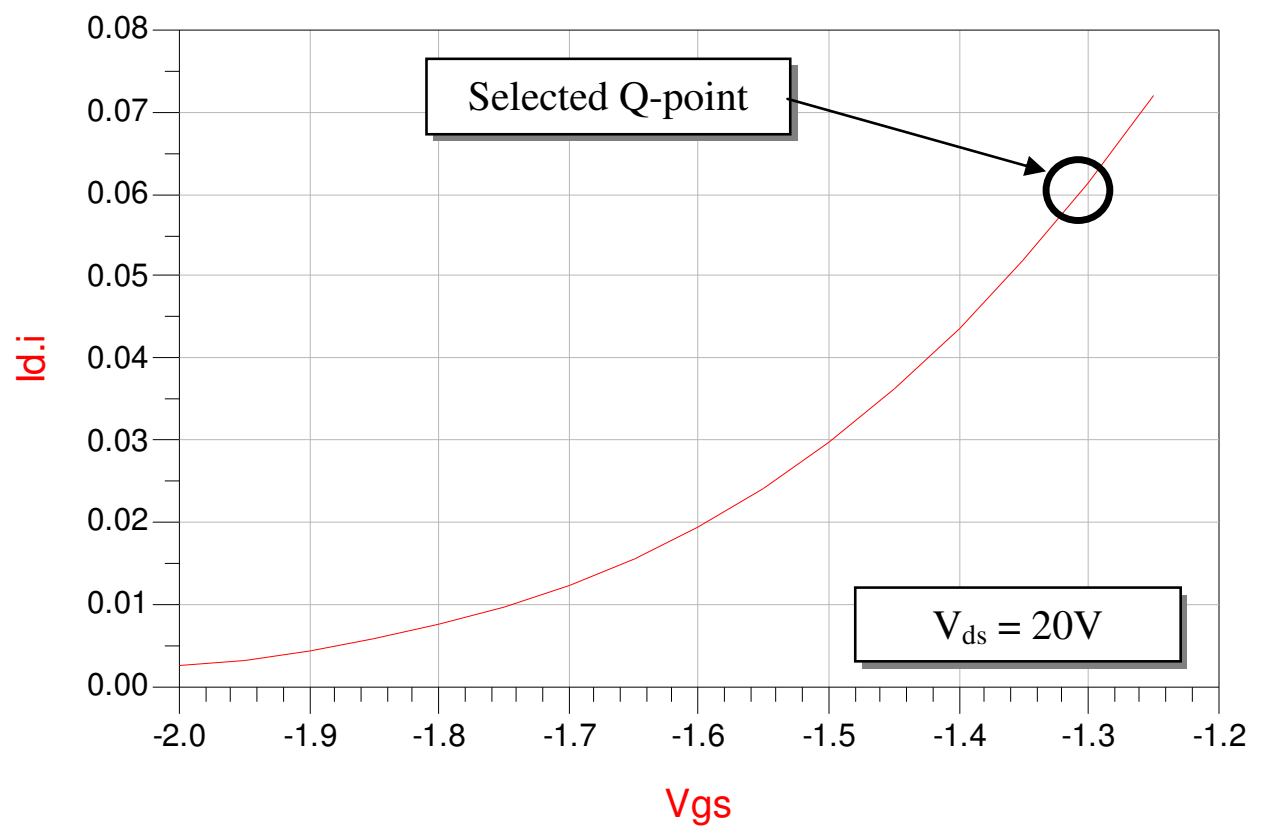

Figure 2.10 ? Single transfer curve at a $\mathrm{V}_{\mathrm{ds}}$ of $20 \mathrm{~V}$. 


\subsubsection{S-parameters}

The schematic used to simulate the S-parameters of the NPTB00004 is shown in Figure 2.11. The bias conditions for the S-parameter simulations are listed below.

- S-parameters: bias conditions

$\circ \quad P_{\text {in }}=0 \mathrm{dBm}$

- Frequency range: $100 \mathrm{MHz}$ to $3 \mathrm{GHz}$

$\circ \quad \mathrm{V}_{\mathrm{gs}}=-1.3 \mathrm{~V}$

$\circ \mathrm{V}_{\mathrm{ds}}=20 \mathrm{~V}$

For the actual S-parameter measurements, two bias tees were used to provide the DC bias network for all DUTs. A HP11612A bias network and a HP33150A bias tee were used as the DC bias networks. A lower limit of $100 \mathrm{MHz}$ used during S-parameter simulation was utilized due to the $100 \mathrm{MHz}$ lower frequency limit of the HP33150A bias tee. Because the schematic for the HP33150A could not be found, the components used in the HP11612A were used to represent both bias networks in the simulation circuit of Figure 2.11. The schematic of Figure 2.11 does not take into account line lengths from coaxial cables or the bias networks when simulating the S-parameters.

The simulated S-parameters are shown in Figure 2.12. At the selected Q-point, the drain current and gate current were simulated to be $61.47 \mathrm{~mA}$ and $21.89 \mathrm{fA}$, respectively. A maximum obtainable gain of approximately $25.58 \mathrm{~dB}(19 \mathrm{~V} / \mathrm{V})$ was simulated at $100 \mathrm{MHz}$ while a reverse transmission gain of $-27.54 \mathrm{~dB}(0.042 \mathrm{~V} / \mathrm{V})$ was simulated at 1.2GHz. The drain impedance was observed to be capacitive over the simulated frequency range. The gate impedance, however, becomes inductive at approximately $1.88 \mathrm{GHz}\left(\mathrm{Z}_{\text {gate }}=4.95+\mathrm{j} 0.02 \Omega\right)$. 


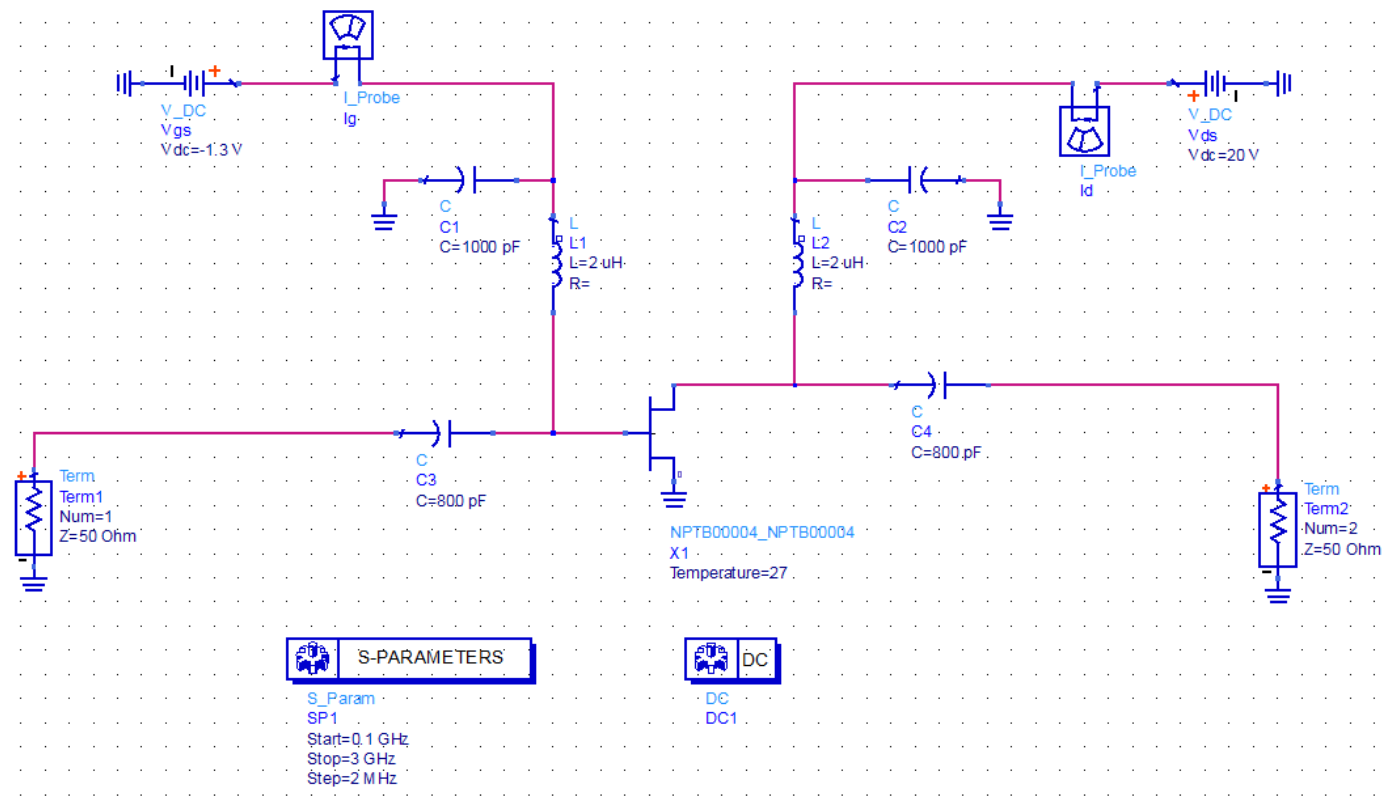

Figure 2.11 ? Agilent ADS schematic used for RF simulations.
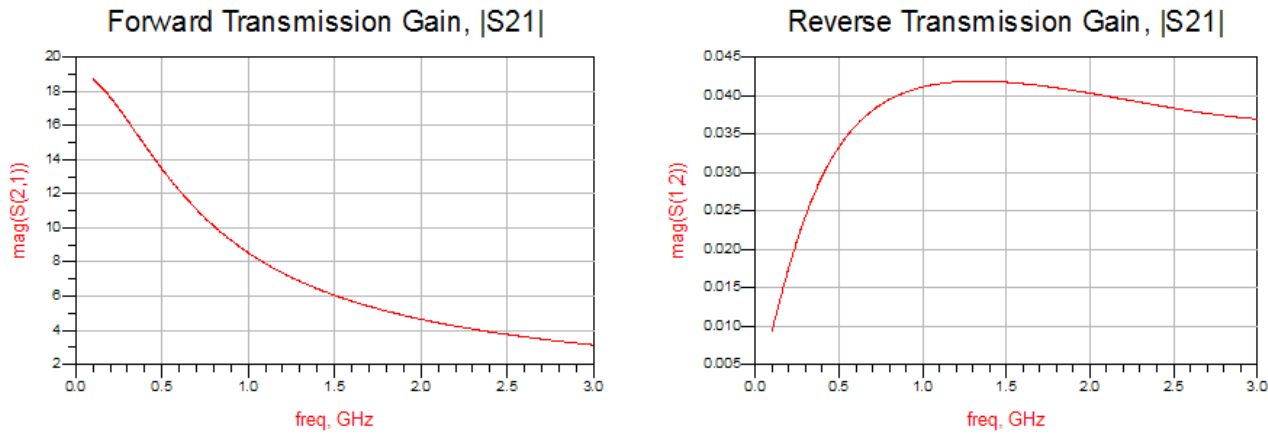

\begin{tabular}{|c|c|c|}
\hline freq & Id.i & Ig.i \\
\hline $0.0000 \mathrm{~Hz}$ & $61.47 \mathrm{~mA}$ & $-21.89 \mathrm{fA}$ \\
\hline
\end{tabular}

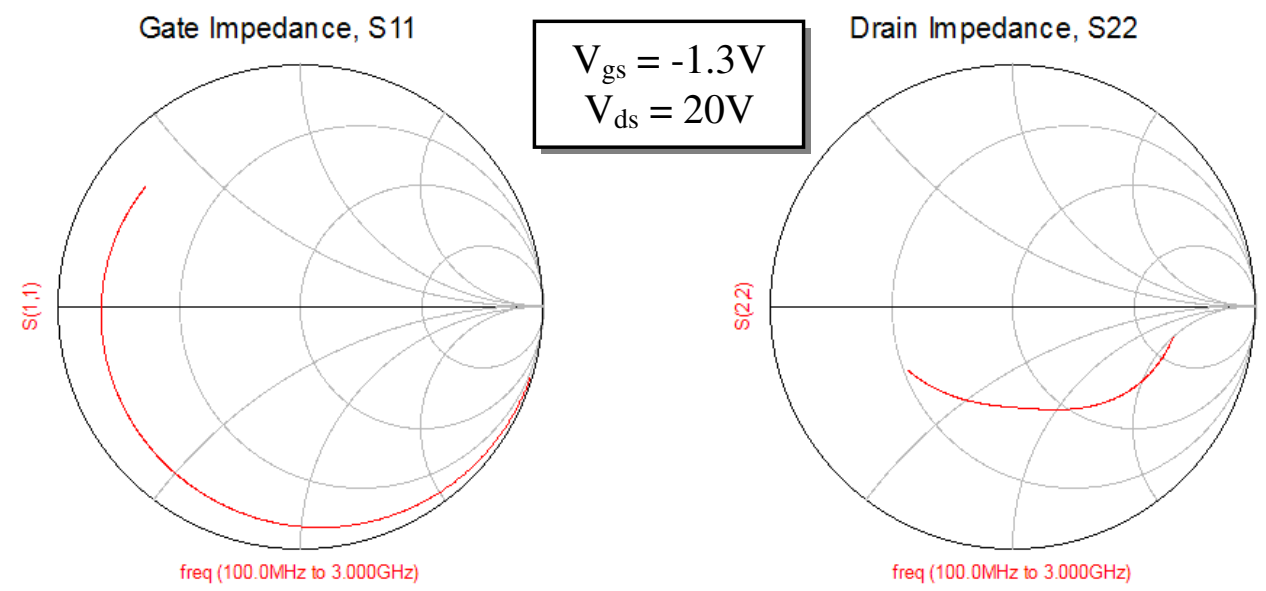

Figure 2.12 $\square$ Simulated S-parameters for the NPTB00004. 


\subsection{DC and RF Characterization of the DUT}

All DC measurements were performed using a HP4155A semiconductor parameter analyzer. Measurements were made using similar bias conditions listed in subsections 2.2.1 and 2.2.2. The integration time used between measurements was $80 \mu$ s. Voltages were applied using BNC cables with $\operatorname{SMA}(\mathrm{F})$ adapters. Figure 2.13 shows the resulting I-V measurement for a single HEMT.

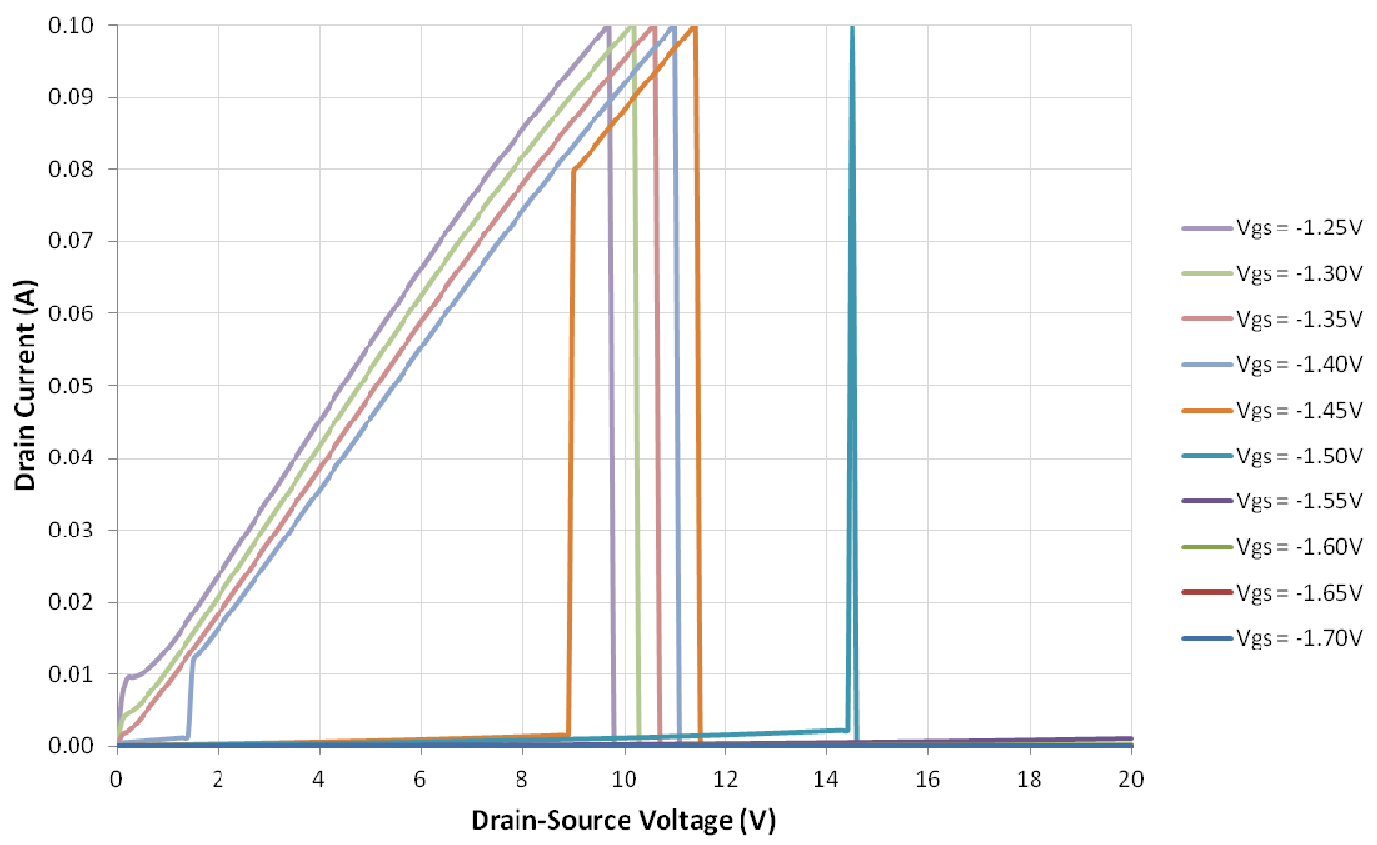

Figure 2.13 ? Initial I-V measurements ( 0 to $20 \mathrm{~V} \mathrm{~V}_{\mathrm{ds}}$ sweep).

As seen above, the measurements do not correlate to the simulated I-V measurements of Figure 2.7. One set of issues that may be linked to the observation above is the presence of oscillations. To dampen the circuit and prevent oscillations, a $200 \Omega$ resistor was required in the gate-feed path to suppress oscillations [21]. A $200 \Omega$ structure, shown in Figure 2.14, was created and used in all proceeding DC measurements. 


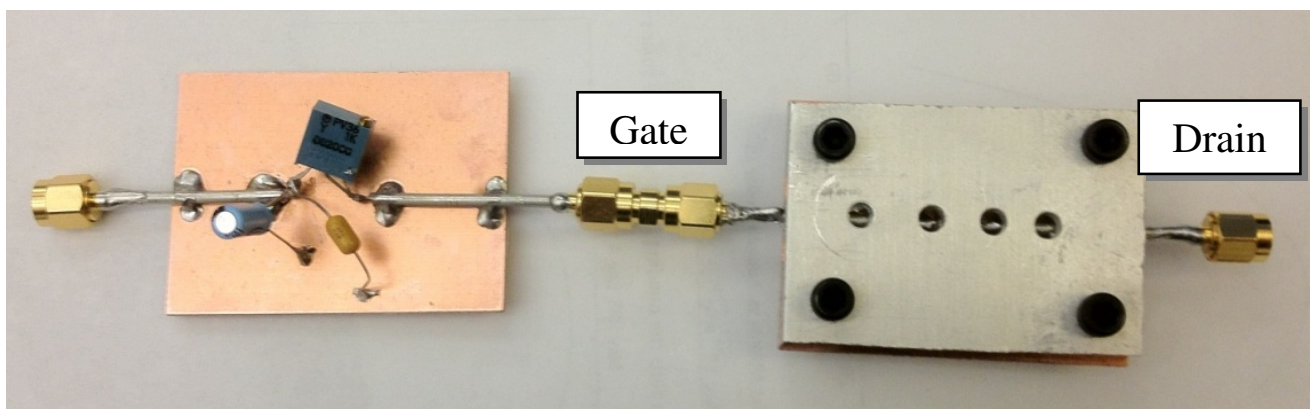

Figure 2.14 $200 \Omega$ structure used to dampen oscillations in the gate feed path.

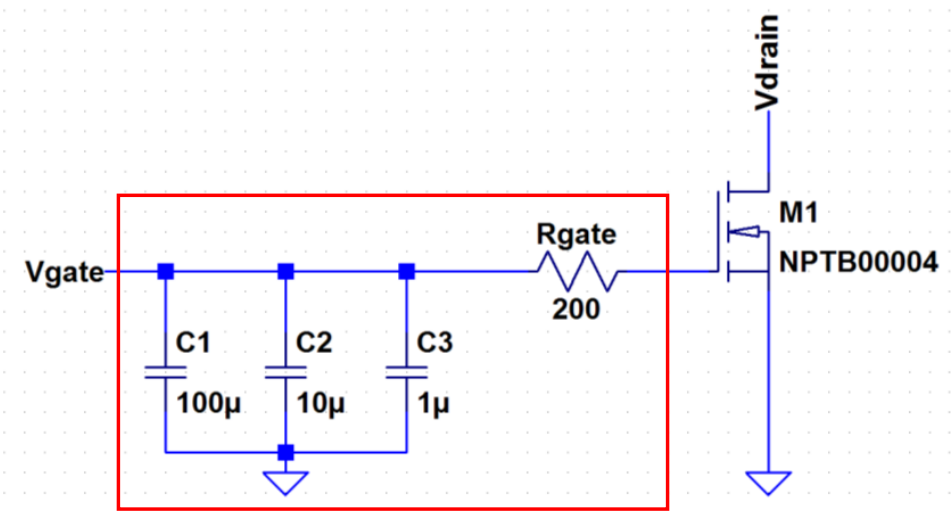

Figure 2.15 ? Schematic representation of the $200 \Omega$ structure.

Transients can also become a factor when applying an excitation source to the device during measurements. An example scenario would be to evaluate the transient response of an RC circuit with two known time constants ( $\tau$ ) and a unit-step source using Equation (2.1) [22].

$$
v(t)=V_{S}\left(1-e^{-\frac{t}{\tau}}\right) u(t), \text { where } \tau=R C
$$

As $\tau$ becomes smaller, the resulting system waveform reaches $V_{S}$ at a much faster rate, or in other words reaches the steady-state voltage quicker. As $\tau$ becomes larger, $v(t)$ takes much longer to reach the steady-state $V_{S}$ value. While a smaller $\tau$ seems desirable, if the system is underdamped (e.g. due to insufficient compensation resistor), the signal 
could overshoot $\mathrm{V}_{\mathrm{S}}$ and experience a ringing nature as the signal approaches steady-state. An example of the aforementioned signals are shown in Figure 2.16.

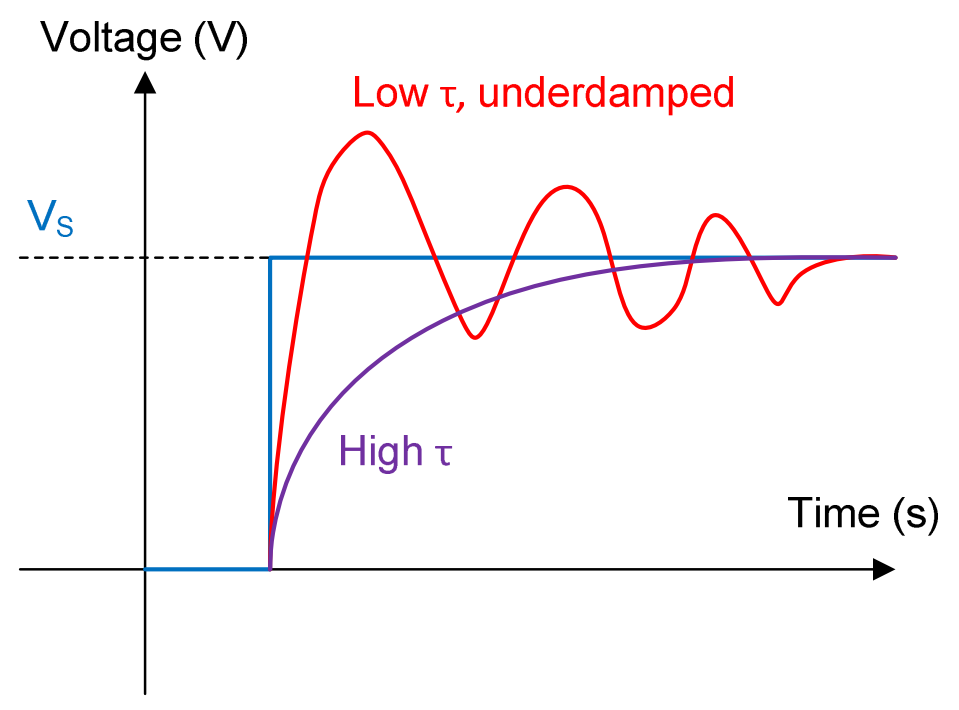

Figure 2.16 ? Effect of time constant on system response to an excitation source.

Based upon the previous analyses, the $200 \Omega$ component was added in the gatefeed path and the integration time was increased from $80 \mu$ s to $266 \mathrm{~ms}$. Due to the increase in measurement time, the increment in $\mathrm{V}_{\mathrm{ds}}$ was increased from $100 \mathrm{mV}$ to $500 \mathrm{mV}$. The resulting I-V measurements are shown in Figure 2.17. An abnormal linear region was observable in the new I-V measurements, where the drain current appears suppressed by some phenomenon. The current reduction in the linear region, or "kink" effect, was determined to be attributed to electron-traps within the HEMT [23], [24]. A second set of I-V curves were generated by sweeping $V_{d s}$ instead from $20 \mathrm{~V}$ down to $0 \mathrm{~V}(500 \mathrm{mV}$ decrements). 


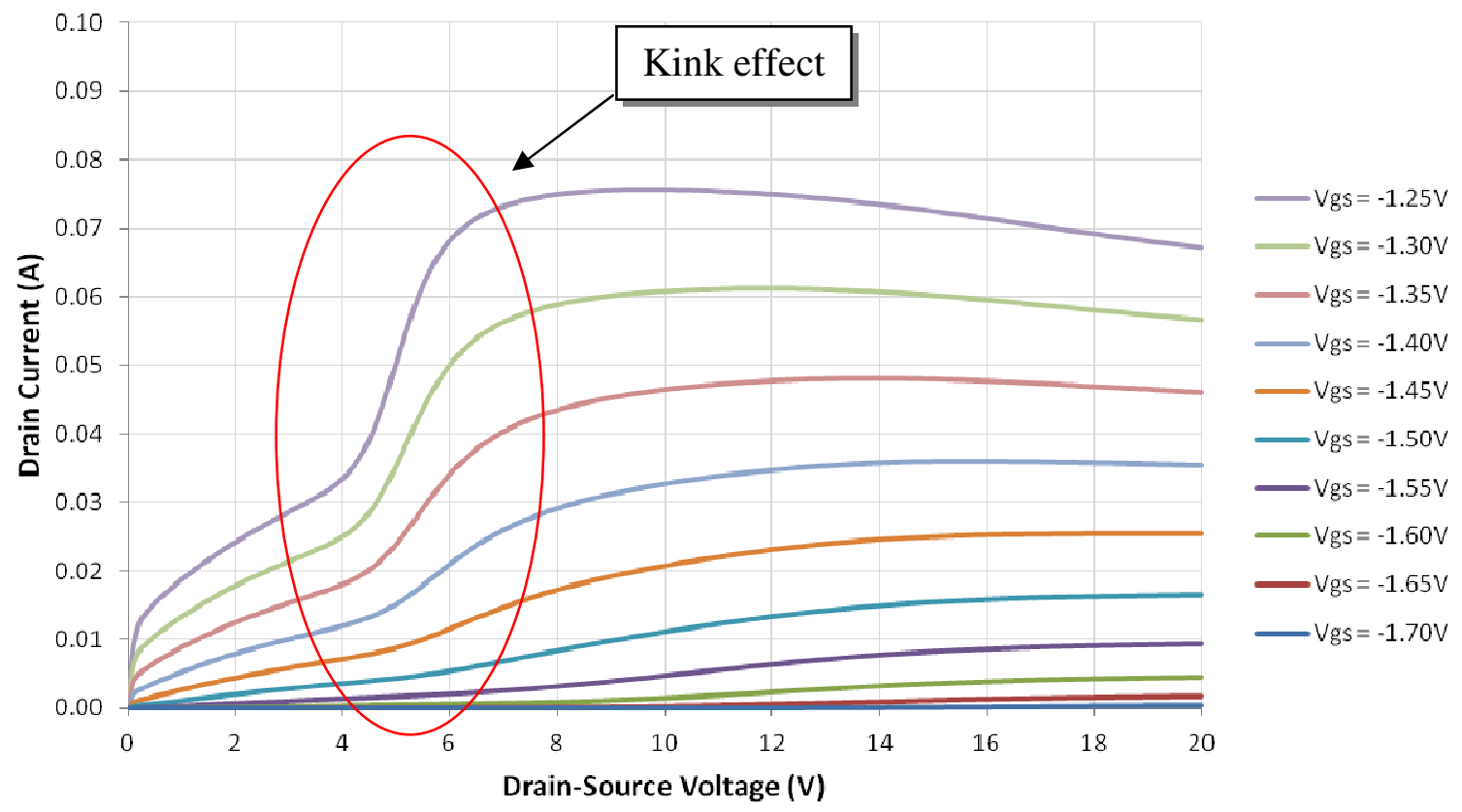

Figure 2.17 I-V measurements using the $200 \Omega$ gate resistor and increased integration time ( 0 to $20 \mathrm{~V} \mathrm{~V}_{\mathrm{ds}}$ sweep).

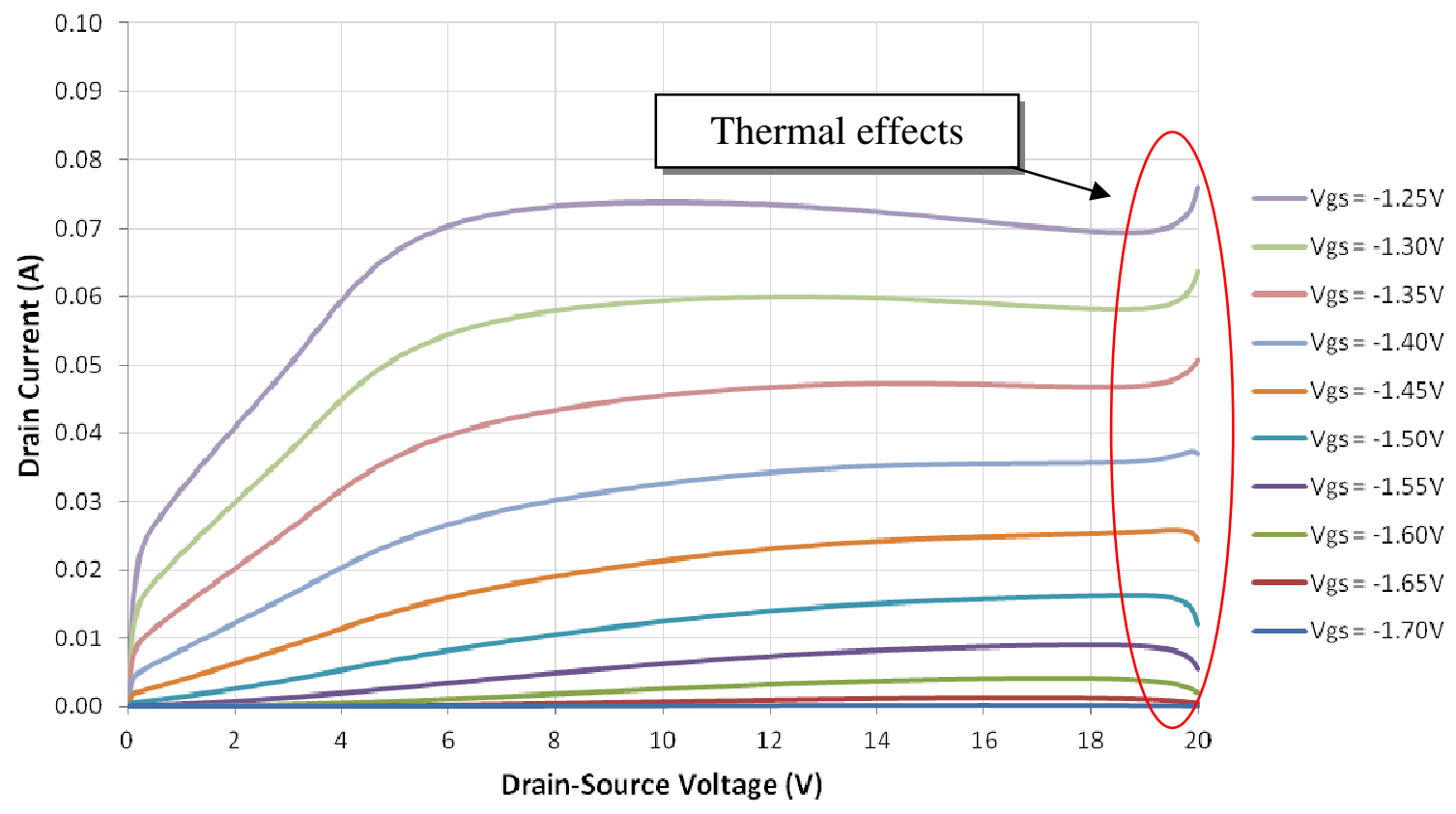

Figure 2.18 ? I-V measurements recorded by sweeping $\mathrm{V}_{\mathrm{ds}}$ from $20 \mathrm{~V}$ to $0 \mathrm{~V}$. 
The new I-V measurements are displayed in Figure 2.18. While the linear region experienced an observable increase in drain current, the characteristics still remain relatively ohmic in nature. Another observation was how the saturation region of each curve tended to curl around $\mathrm{V}_{\mathrm{ds}}=20 \mathrm{~V}$. The curling characteristic could potentially be inherent to the way the device turns on when instantaneously biased at a rather large drain voltage value using the transistor parameter analyzer.

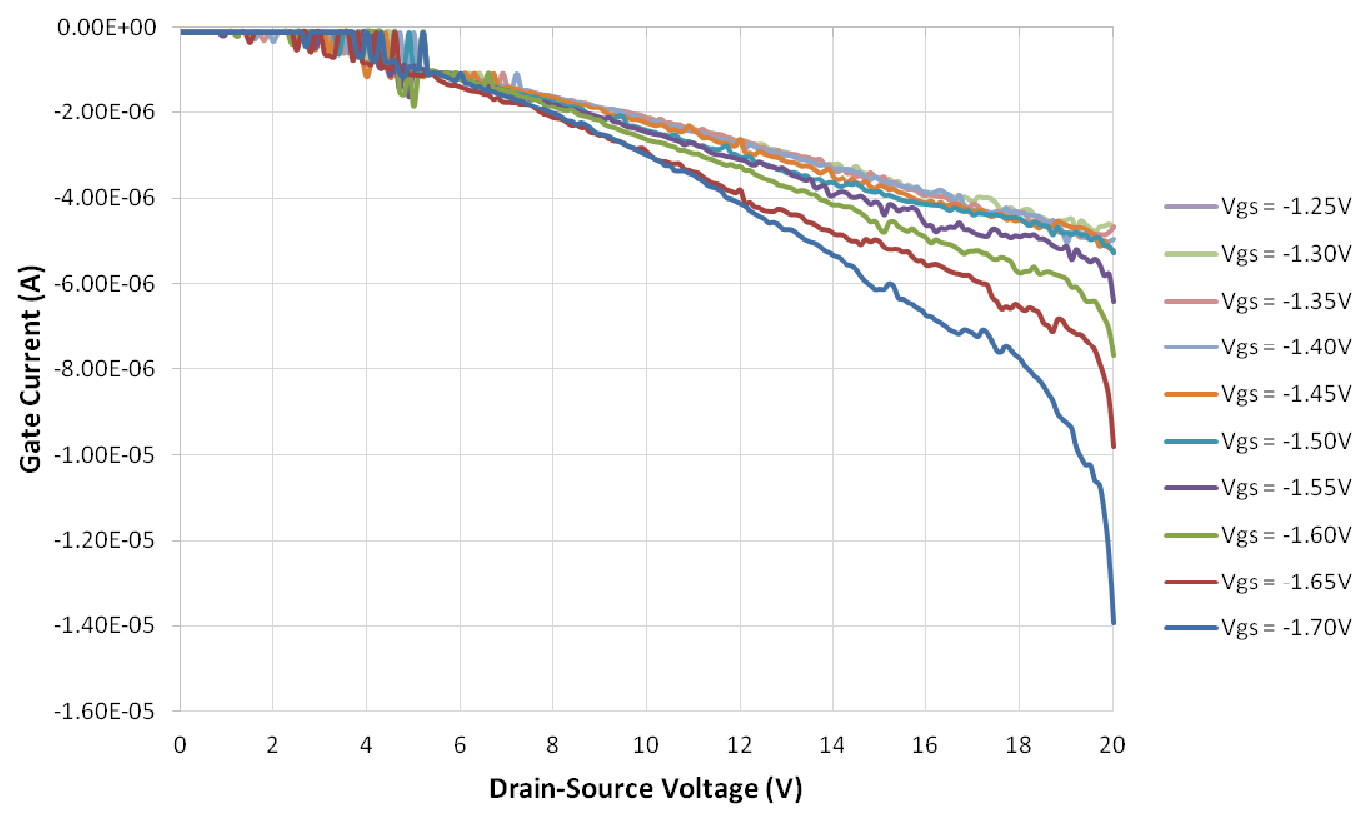

Figure 2.19 $\square$ Gate current measurements.

A family of gate current measurements is show in Figure 2.19 above. As seen in the measurements of Figure 2.19, as the device is set further into the on-state, the effective gate current drawn from the device decreases. It should be noted that the gate current measurements in Figure 2.19 possess a negative polarity because current is being sourced from the HEMT (the same will be seen throughout the entirety of the report). Figure 2.20 shows the measured transfer characteristics for the DUT with a constant $V_{d s}$ bias of $20 \mathrm{~V}$. 


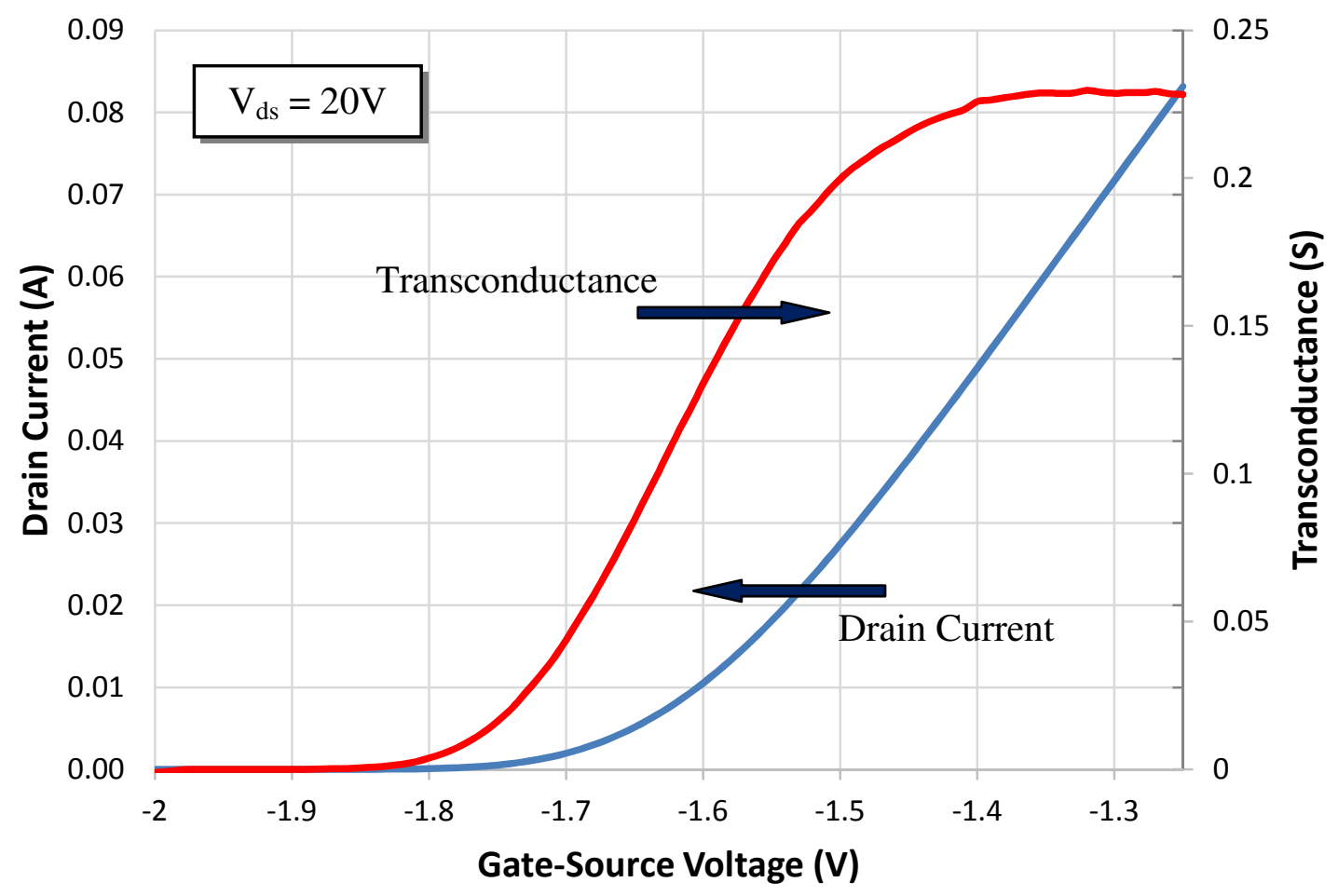

Figure 2.20 $\square$ Measured transfer characteristics.

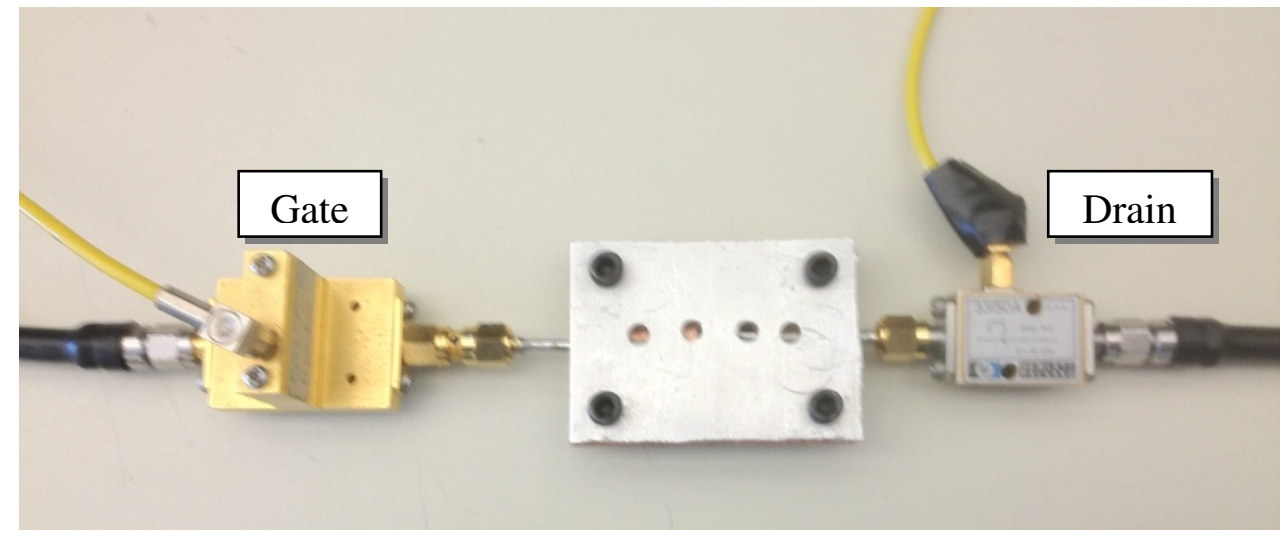

Figure 2.21 ? Bias tees configuration for S-parameter measurements.

The S-parameter measurements were extracted using an Anritsu MS4622B Vector Network Analyzer (VNA) using the bias conditions and bias tees described in Subsection 2.2.3. RF measurements did not include the use of matching networks since parameters such as maximum attainable gain (MSG) and voltage standing-wave ratio 
(VSWR) were negligible figures of merit. A visual demonstrating the connections between the bias tees and the DUT structure is shown in Figure 2.21. Before conducting measurements, reference plane shifts were applied to both the gate and drain transmission lines (Channels 1 and 4, respectively). Reference plane shifts were performed by entering into the VNA the physical length of each line in millimeters and selecting the correct dielectric material used to insulate the outer and inner conductors. An S2P file was extracted from the VNA and imported into Agilent ADS to plot the results (Figure 2.22).
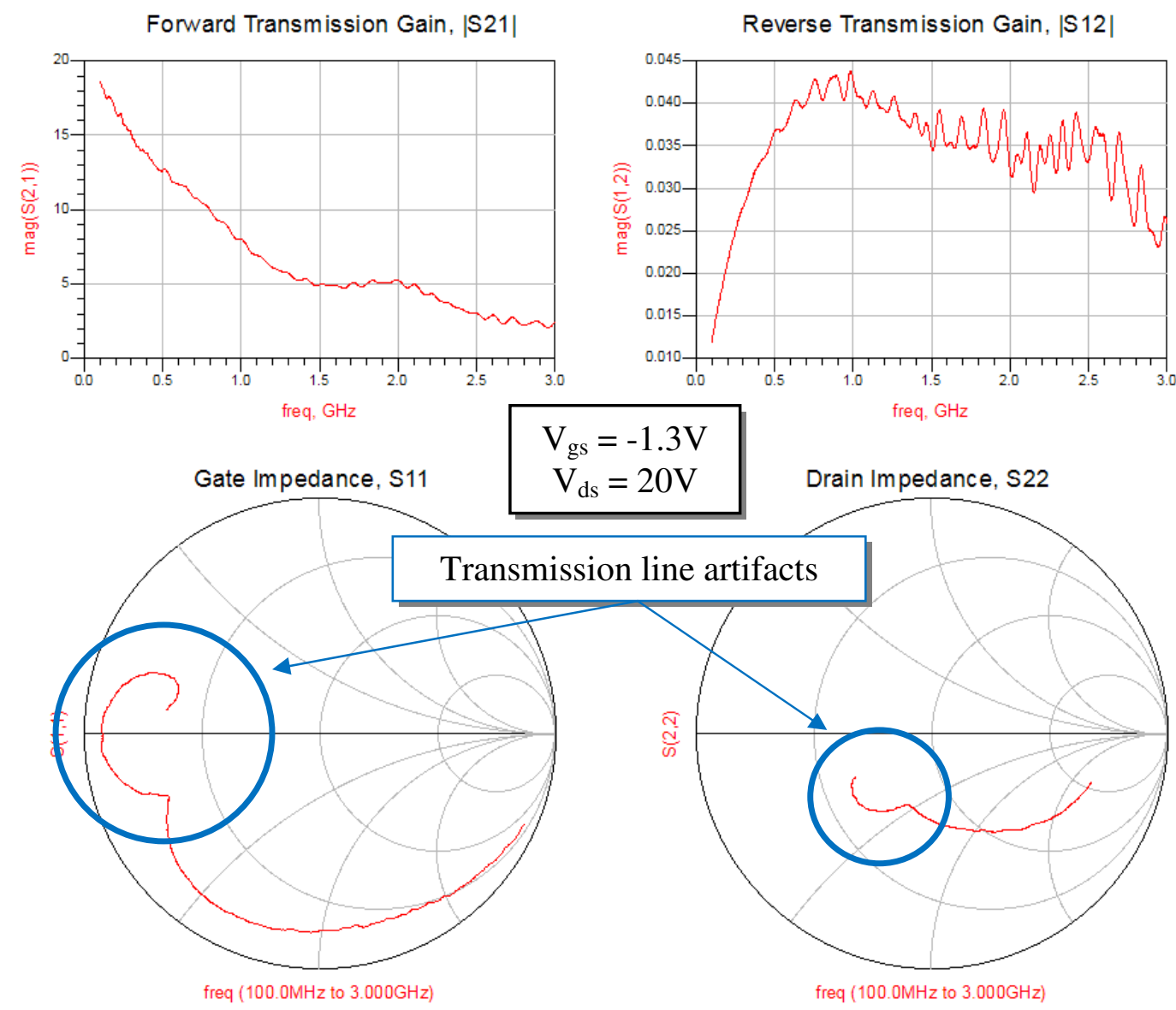

$\mathrm{V}_{\mathrm{gs}}=-1.3 \mathrm{~V}$
$\mathrm{~V}_{\mathrm{ds}}=20 \mathrm{~V}$

Drain Impedance, S22

ion line artifacts

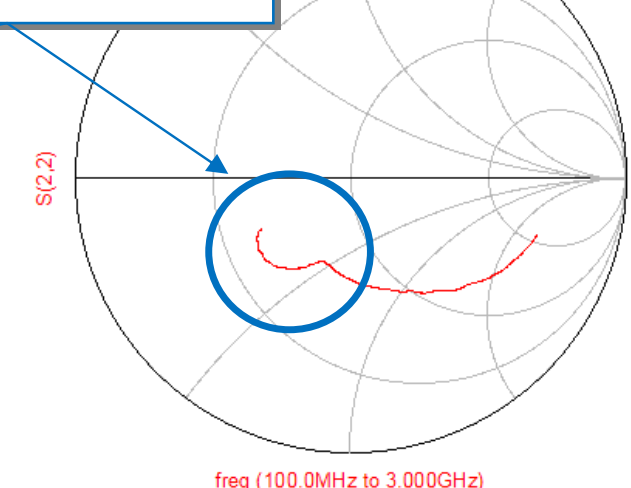

Figure 2.22 $\square$ S-parameter measurements. 
From Figure 2.22, it can be seen how the input and output impedance measurements show a type of wrapping nature at high frequencies. The wrapping nature was determined to be an artifact created at high frequencies due to the inductances inherent in the transmission lines used for each DUT's structure.

\subsection{Test Plans - Overview}

Table 2.1 lists the test plans for four groups of HEMTs (approximately 60 transistors) involved in the GaN-on-Si research and Table 2.2 lists the instruments and software tools used during the experimental process. The first experiment involved assessing the HEMT's critical voltage, or the gate bias voltage condition at which a large amount gate leakage current is observed. Two transistor configurations are evaluated in the step-stress procedures. DC and RF characteristics were analyzed before and after high voltage biases were applied to the DUTs. After performing the step-stress tests, the devices were then monitored to determine if any altered characteristics were recoverable post-stress.

Experiment 2 involved assessing the DC and RF parameters of the DUTs under increasing back-plate temperatures. Recovery analyses were then performed after thermally stressing each HEMT. A second set of experiments were then conducted to assess how the critical voltage determined in Experiment 1 varies over increasing backplate temperatures. Experiment 3 investigated the nature of electron trapping and detrapping mechanisms within the GaN devices under normal operation and during high field exposure. Finally, the goal of Experiment 4 was to map alterations in the test transistors' RF performances to their equivalent small-signal models after exposure to 


\title{
high-field stress. Using tuning parameters in Agilent Advanced Design System (ADS),
}

\author{
small-signal models were developed for the stressed HEMTs.
}

Table 2.1 ? Overview of HEMT experiments.

\begin{tabular}{|c|c|c|c|c|}
\hline \multirow[t]{2}{*}{ Exp. \# } & \multirow{2}{*}{$\begin{array}{l}\text { Chapter \# } \\
\text { and Page \# }\end{array}$} & \multicolumn{3}{|c|}{ Experimental Details } \\
\hline & & Exp. Name & Sub-Exp. Name & Testing Overview \\
\hline \multirow[b]{2}{*}{1} & \multirow[b]{2}{*}{3 (pg. 32) } & \multirow[b]{2}{*}{$\begin{array}{l}\text { High Electric } \\
\text { Field Stress } \\
\text { Analyses }\end{array}$} & $\begin{array}{l}\text { Asymmetric Electric Field } \\
\text { Stress }\end{array}$ & $\begin{array}{l}\text { Evaluation of each DUTs DC and RF } \\
\text { characteristics before and after a high drain } \\
\text { voltage bias is applied while the device is in } \\
\text { the off-state }\left(\mathrm{V}_{\mathrm{gs}}=-5 \mathrm{~V}\right) \text {. The high drain } \\
\text { bias will create a large vertical electric field } \\
\text { parallel to the substrate of the devices. Main } \\
\text { analytical focus is the critical voltage with } \\
\text { respect to the drain bias. }\end{array}$ \\
\hline & & & $\begin{array}{l}\text { Symmetric Electric Field } \\
\text { Stress }\end{array}$ & $\begin{array}{l}\text { Similar evaluations done in the asymmetric } \\
\text { E-field stress test will be performed while } \\
\text { the device is in the off-state }\left(\mathrm{V}_{\mathrm{d}}=0 \mathrm{~V}\right) \text {. A } \\
\text { high negative gate bias will be applied to } \\
\text { induce a large E-field perpendicular to the } \\
\text { substrate of the devices. Main analytical } \\
\text { focus is the critical voltage with respect to } \\
\text { the gate bias. Additional analyses are } \\
\text { performed to evaluate the stressed devices } \\
\text { over relaxation periods (no excitation). }\end{array}$ \\
\hline \multirow{2}{*}{2} & \multirow{2}{*}{4 (pg. 49) } & \multirow{2}{*}{$\begin{array}{l}\text { Temperature } \\
\text { Characterization }\end{array}$} & $\begin{array}{l}\text { Effect of Temperature on } \\
\text { General Device } \\
\text { Performance }\end{array}$ & $\begin{array}{l}\text { Evaluation of } \mathrm{DC} \text { parameters, } \mathrm{RF} \\
\text { parameters, and junction temperature under } \\
\text { various back-plate temperatures. }\end{array}$ \\
\hline & & & $\begin{array}{l}\text { Effect of Temperature on } \\
\text { the Critical Voltage }\end{array}$ & $\begin{array}{l}\text { The devices' critical voltages will be } \\
\text { measured under thermal strain and tracked } \\
\text { to observe whether or not a temperature } \\
\text { dependence exists. }\end{array}$ \\
\hline \multirow{2}{*}{3} & \multirow{2}{*}{5 (pg. 62) } & \multirow{2}{*}{$\begin{array}{l}\text { Electron } \\
\text { Trapping and } \\
\text { Detrapping }\end{array}$} & $\begin{array}{l}\text { Trapping Experiment A: } \\
\text { On-State Operation }\end{array}$ & $\begin{array}{l}\text { The test will analyze trapping mechanisms } \\
\text { after operating the device in the on-state. } \\
\text { Thermal excitation is used to aid in the } \\
\text { detrapping of electrons. }\end{array}$ \\
\hline & & & $\begin{array}{l}\text { Trapping Experiment B: } \\
\text { High Electric Field Stress }\end{array}$ & $\begin{array}{l}\text { The test will investigate potential trapping } \\
\text { mechanisms induced through high-field } \\
\text { stress. Thermal excitation is used to aid in } \\
\text { the detrapping of electrons. }\end{array}$ \\
\hline 4 & 6 (pg. 70) & $\begin{array}{l}\text { Small-Signal } \\
\text { Analysis }\end{array}$ & $\sim$ & $\begin{array}{l}\text { Creation of a small-signal model to evaluate } \\
\text { how high-field stress alters the small-signal } \\
\text { parameters of the DUTs. }\end{array}$ \\
\hline
\end{tabular}


Table 2.2 [ Key instruments and software used in experiments.

\begin{tabular}{|c|c|}
\hline \multicolumn{2}{|c|}{ Instrument and Software Details } \\
\hline Name & Purpose \\
\hline $\begin{array}{c}\text { Agilent Technologies 6555A 120V, } \\
\text { 4A DC Power Supply }\end{array}$ & Provide high-voltage bias from $0-120 \mathrm{~V}$. \\
\hline $\begin{array}{l}\text { Agilent Technologies 34401A } \\
\text { Digital Multimeter, } 6 \text { 1/2 Digit }\end{array}$ & Current monitoring. \\
\hline $\begin{array}{l}\text { HP4155A Semiconductor Parameter } \\
\text { Analyzer }\end{array}$ & Measurement of I-V and transfer characteristics. \\
\hline $\begin{array}{l}\text { ILX Lightwave LDC-3744C Laser } \\
\text { Diode Controller }\end{array}$ & Thermoelectric cooler controller and thermistor monitor. \\
\hline $\begin{array}{l}\text { Anritsu MS4622B } 0.01-3 \mathrm{GHz} \\
\text { Vector Network Analyzer }\end{array}$ & S-parameter measurements. \\
\hline TE HY3003-3 DC Power Supply & Provides drain or gate bias voltage. \\
\hline $\begin{array}{l}\text { HP11612A Bias Network 45MHz- } \\
26.5 \mathrm{GHz}\end{array}$ & DC bias network used in S-parameter measurements. \\
\hline HP33150A Bias Tee 0.1-18GHz & DC bias network used in S-parameter measurements. \\
\hline Agilent Advanced Design System & S-parameter analysis and small-signal modeling. \\
\hline National Instruments LabVIEW & Automation of instruments. \\
\hline
\end{tabular}




\section{Experiment 1: High Electric Field Stress Analyses}

\subsection{Asymmetric Electric Field Stress}

\subsubsection{Setup}

Figure 3.1 shows the circuit configuration used to apply a high asymmetric electric field across the device. The electric field applied to the device is deemed "asymmetric" because stress is only applied to the drain of the transistor, creating a strong electric field gradient (horizontal field) between the gate and drain regions. The asymmetric field stress procedure first involved setting $\mathrm{V}_{\mathrm{gs}}$ to a constant $-5 \mathrm{~V}$ to set the device in the off-state (no drain current conduction, no power dissipation). $\mathrm{V}_{\mathrm{ds}}$ was then stepped from $30 \mathrm{~V}$ to $120 \mathrm{~V}$ in $1 \mathrm{~V}$ increments every minute. Gate leakage current and drain current were measured during the step-stressing procedure. A HP6555A DC Power Supply was used to supply the large $\mathrm{V}_{\mathrm{ds}}$ voltage biases.

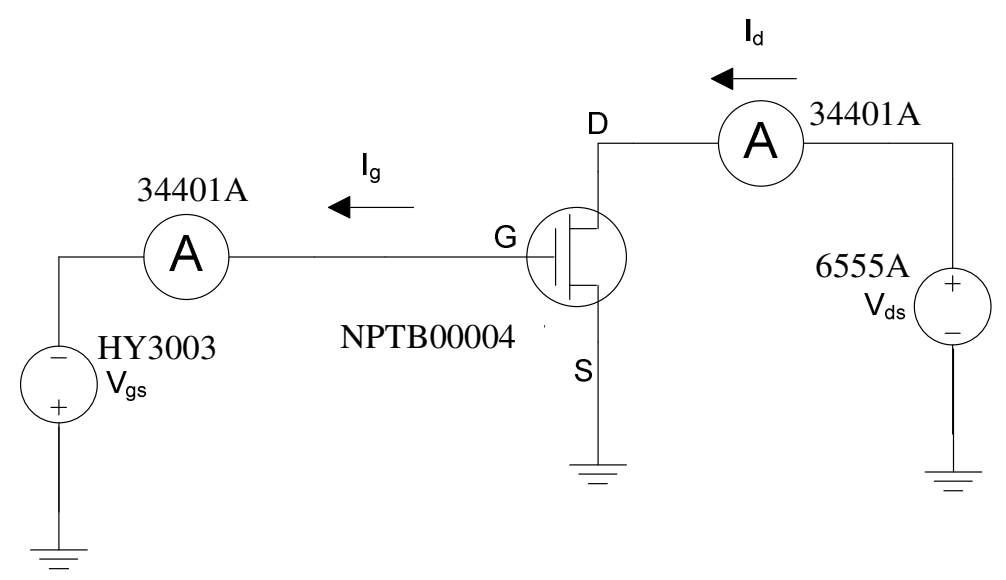

Figure 3.1 ? Circuit configuration for the horizontal E-field stress experiments. 


\subsubsection{Results and Analyses}

Figure 3.2 shows the resulting gate leakage current measurement recorded using the step-stress procedure outlined in Subsection 3.1.1. Over the entire $V_{\mathrm{ds}}$ incremental span, the gate leakage current of the DUT increased from $1.5 \mu \mathrm{A}$ to $2.6 \mu \mathrm{A}$.

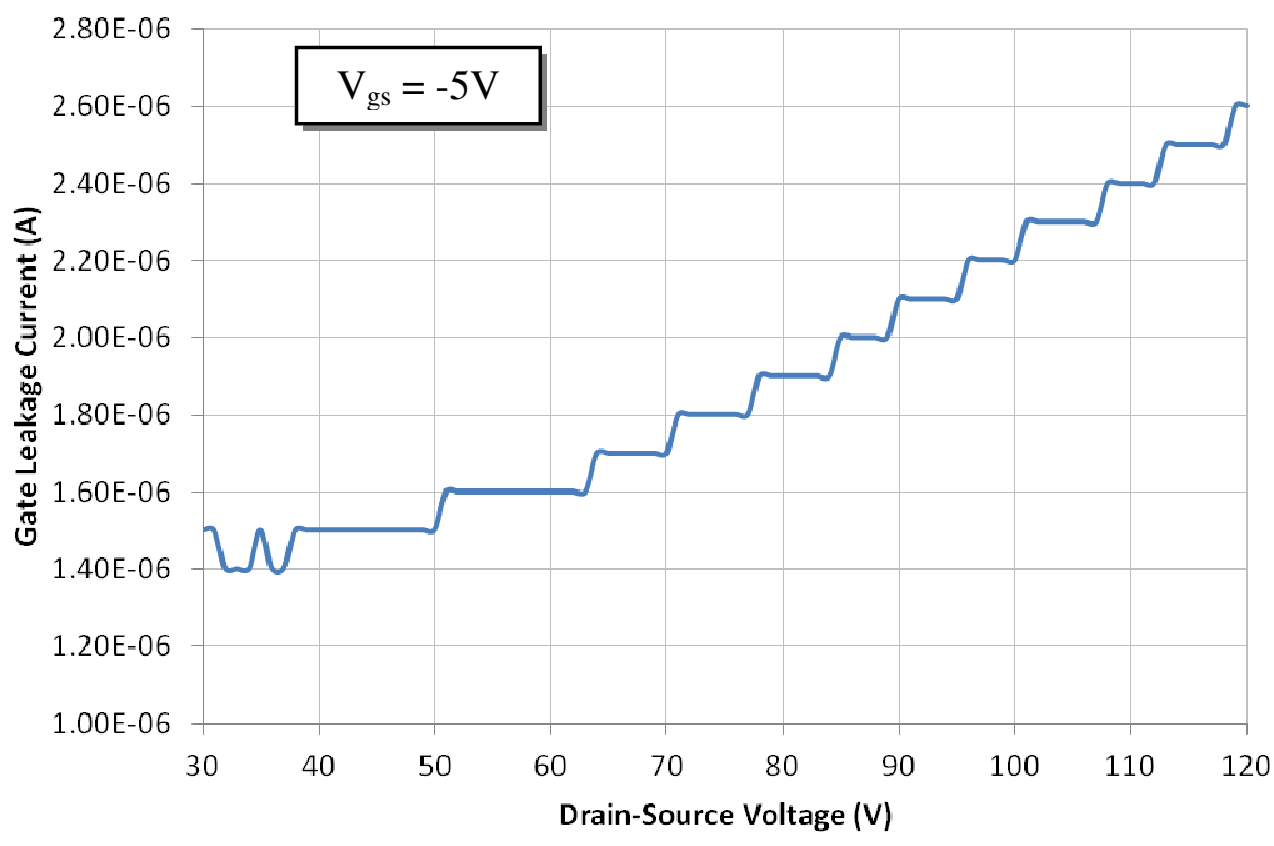

Figure 3.2 ? Gate leakage current measurement results during high asymmetric electric field stress.

While there was an observable (yet negligible) change in the gate leakage current measurements, there were very few changes between each DUT's pre-stress and poststress DC and RF characteristics. The only noticeable change was to each device's gate current. Figure 3.3 shows how the device's overall gate current reduces after being exposed to high drain voltage biases. 


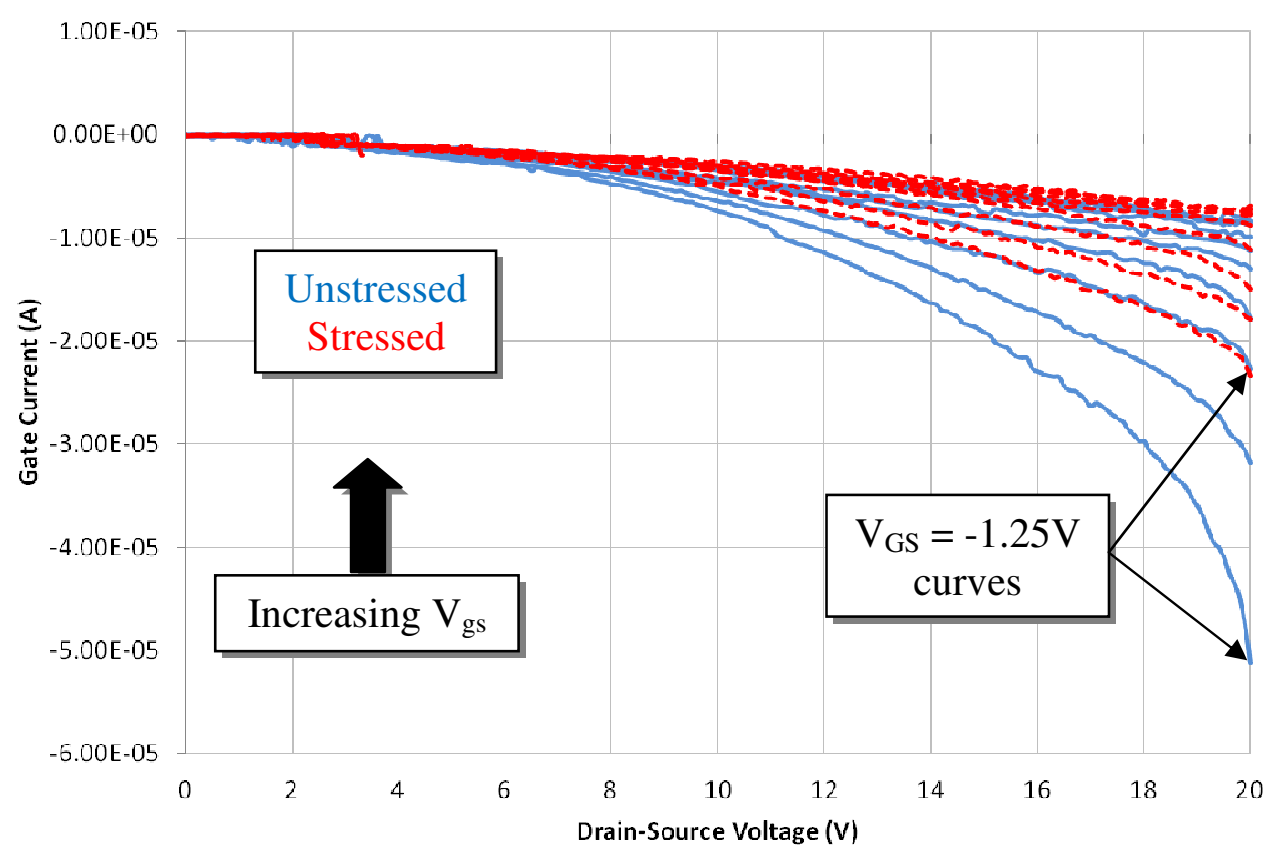

Figure 3.3 ? Gate current measurements before and after high asymmetric electric field stress.

\subsection{Symmetric Electric Field Stress}

\subsubsection{Setup}

Figure 3.4 shows the circuit configuration used to apply a high symmetric electric

field across the device. In this configuration, the applied electric field is evenly distributed throughout the device due to the drain and source being joined together and occurs vertical to the substrate of the device. The symmetric field stress procedure involved tying the drain and source terminals to ground, which set the device in the offstate (no drain current conduction, no power dissipation). The first test, known as a stepstress procedure, involved stepping $\mathrm{V}_{\mathrm{gs}}$ from $-10 \mathrm{~V}$ to $-70 \mathrm{~V}$ in $1 \mathrm{~V}$ decrements every minute. Gate leakage current was measured during the step-stressing procedure. After identifying the critical voltage $\left(\mathrm{V}_{\text {crit }}\right)$ of the HEMT, each DUT was then stressed at a 
constant $\mathrm{V}_{\mathrm{gs}}=\mathrm{V}_{\text {crit }}$ bias while gate leakage current was monitored. The constant $\mathrm{V}_{\mathrm{gs}}$ testscenario was performed until gate leakage current ceased to change or device failure was experienced. A HP6555A DC Power Supply was used to supply the large $\mathrm{V}_{\mathrm{gs}}$ voltage biases.

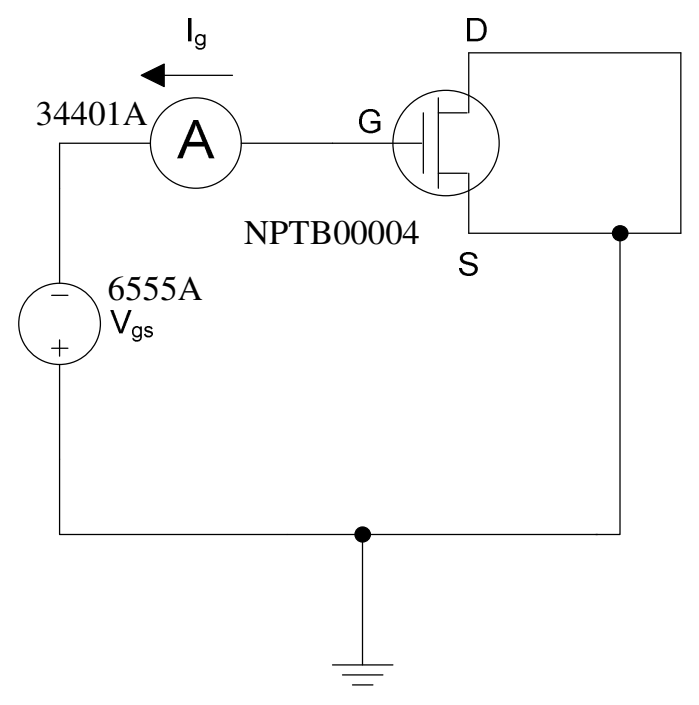

Figure 3.4 [ Circuit configuration for the high symmetric electric field stress experiments.

\subsubsection{Results and Analyses}

Two transistors were used during the initial symmetric field stress analyses, and their resulting gate leakage current measurements are shown in Figure 3.5. As $V_{\mathrm{gs}}$ was stepped more negative over time, an observed rise in gate leakage current was apparent around $\mathrm{V}_{\mathrm{gs}}=-50 \mathrm{~V}$. As $\mathrm{V}_{\mathrm{gs}}$ approached $-70 \mathrm{~V}$ (estimated $\mathrm{V}_{\text {crit }}$, indicated by a dashed red line in Figure 3.5), a drastic increase in gate leakage current was experienced. An absolute increase of the gate-source voltage beyond $-70 \mathrm{~V}$ either resulted in immediate device failure, or gradual device breakdown leading to imminent device failure.

Performing the symmetric field step-stress experiments with a larger pool of HEMTs resulted in similar gate leakage current characteristics around $\mathrm{V}_{\mathrm{gs}}=-70 \mathrm{~V}$. 
Figure 3.6 shows a representative gate leakage trend for the DUTs, where a sudden rise in gate leakage current was experienced as $V_{g s}$ approached $V_{\text {crit. }}$. An increase in gate leakage current was determined to be attributed to the conduction of electrons between the gate and the channel, which in turn was a result of possible defect formations induced by the inverse piezoelectric effect [25].

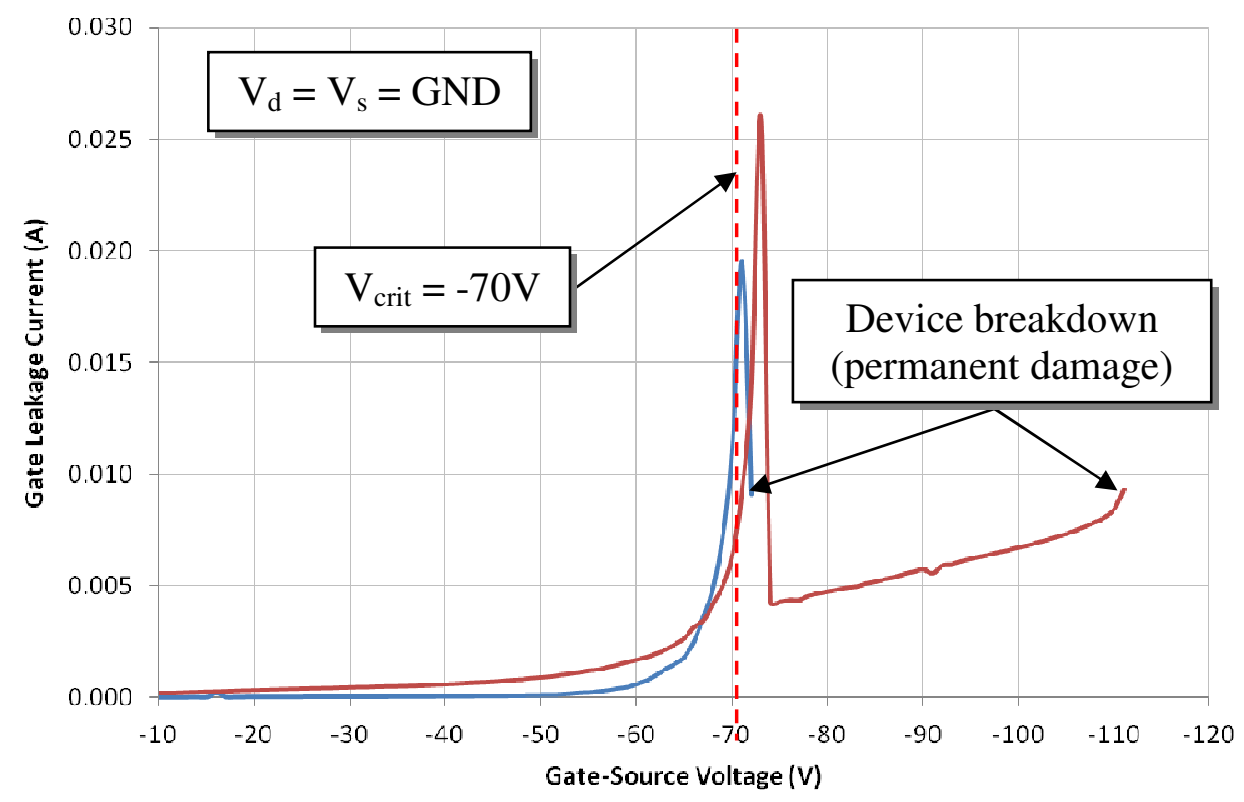

Figure 3.5 ? $\mathrm{V}_{\text {crit }}$ observations using the high symmetric electric field step-stress procedure. 


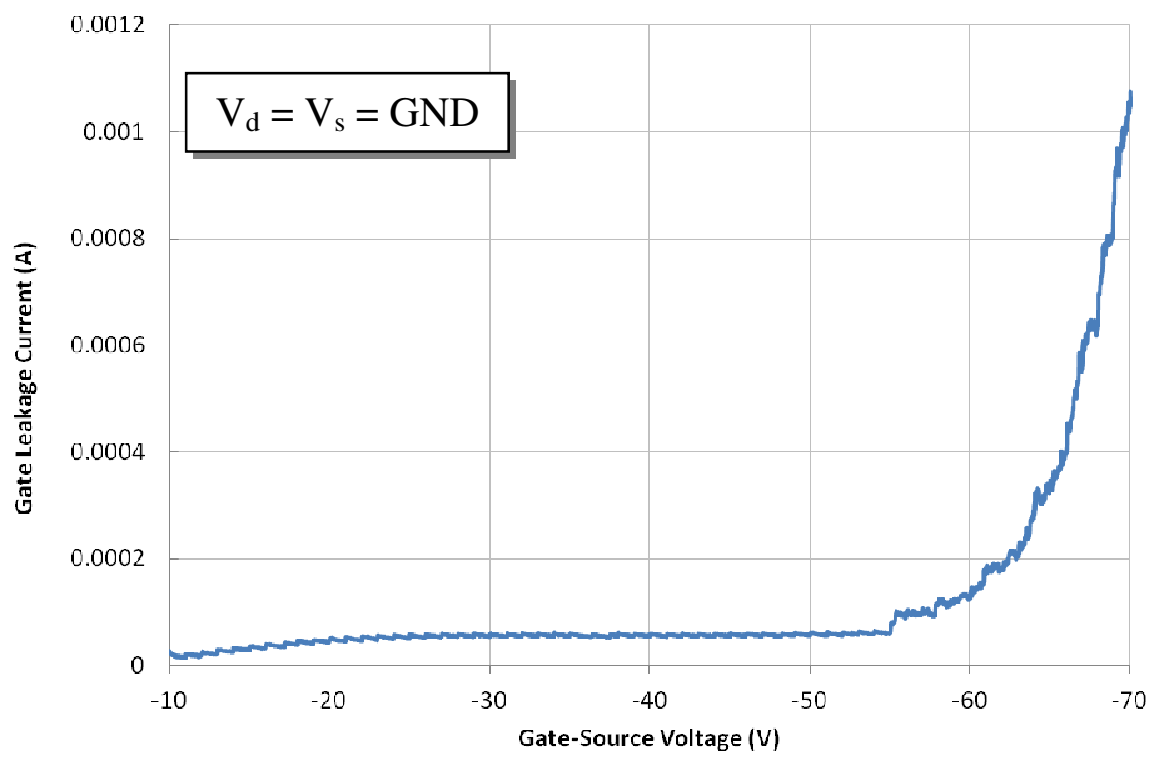

Figure 3.6 ? Typical gate leakage characteristic as the gate-source voltage approaches the established $\mathrm{V}_{\text {crit }}$.

An additional experiment was performed where the gate-source voltage was left at a constant $-70 \mathrm{~V}$ while gate leakage current was monitored over time. A representative data set resulting from the aforementioned experiment is shown in Figure 3.7. As seen, a relatively linear trend in increased gate leakage current was seen over time. A common observation from the experiments was that device failure was found to occur when the gate leakage current was either equal to or greater than $10 \mathrm{~mA}$ at $\mathrm{V}_{\mathrm{gs}}=-70 \mathrm{~V}$, equating to roughly $0.7 \mathrm{~W}$. The time it took to reach this limit, however, varied between devices. All proceeding symmetric step-stress experiments utilized the $10 \mathrm{~mA}$ current-limit to prevent device failure from taking place.

Because of the linear nature of the rising gate leakage current seen in Figure 3.7, a linear regression could have been used to determine the average time until device failure. However, the time-to-failure varied from device to device. While the DUT in Figure 3.7 
reached $I_{g}$, leak in approximately 6.5 minutes, another device could have taken up to an hour to reach the same gate leakage current value.

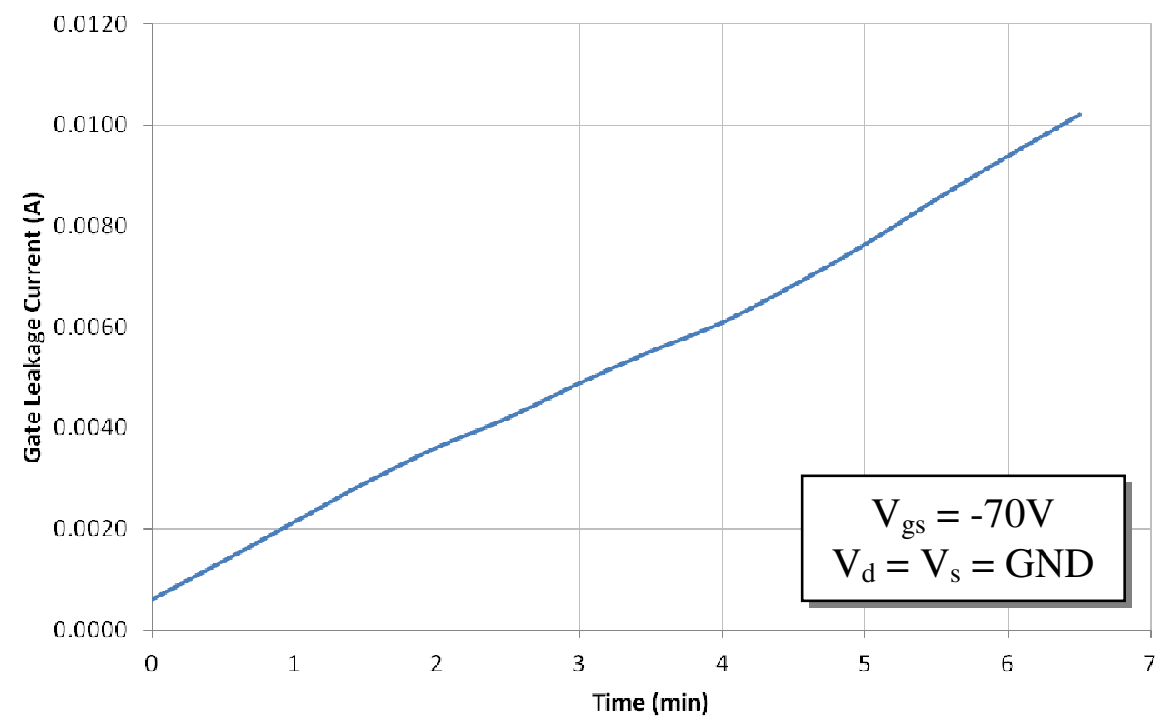

Figure 3.7 ? Gate leakage current characteristic over time as $\mathrm{V}_{\mathrm{gs}}=\mathrm{V}_{\text {crit. }}$.

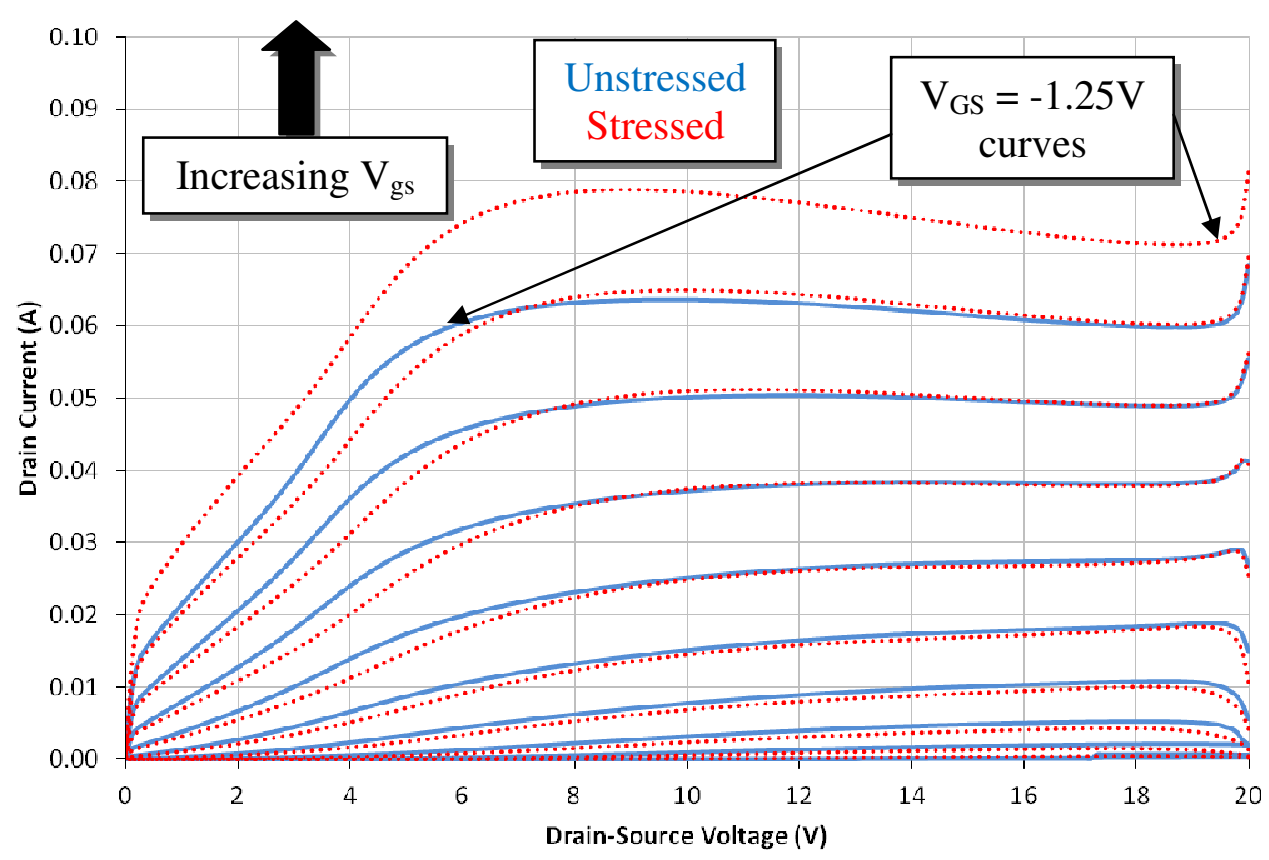

Figure 3.8 ? Recorded I-V characteristics before and after high symmetric electric field step-stress procedure. 
Figure 3.8 shows the resulting I-V characteristics of a representative DUT before and after being subjected to high-field stress. Prior to performing the experiment, it was hypothesized that the saturation drain current, $\mathrm{I}_{\mathrm{d}}$ sat, would decrease as a result of $2 \mathrm{DEG}$ depletion due to trapped electrons in the $\mathrm{AlGaN}$ barrier and defect formation through the inverse piezoelectric effect [10]. The results from Figure 3.8 instead show an overall increase in $\mathrm{I}_{\mathrm{d} \text {, sat. }}$. For example, for $\mathrm{V}_{\mathrm{gs}}=-1.25 \mathrm{~V}, \mathrm{I}_{\mathrm{d} \text {, sat }}$ increased by approximately $14 \%$ from the unstressed value.

A uniaxial tensile strain experiment, performed in [26], determined that a sapphire-substrate HEMT with two $\mathrm{SiN}_{\mathrm{X}}$ passivation layers undergoing a symmetric distribution of mechanical strain experienced an overall increase in $\mathrm{I}_{\mathrm{d} \text {, sat. }}$. The increase in saturation drain current seen in Figure 3.8 could then be attributed to the inverse piezoelectric effect, which provides a symmetric tensile strain to the heterostructure due to the induced symmetric electric field. Research performed in [24] also found that highfield stress promoted drain current recovery, which was determined to be a result of electrons becoming detrapped within the bulk of the device and aided in the recovery of the 2DEG carrier concentration. Further analysis on electron trapping and detrapping will be covered in Chapter 5 . 


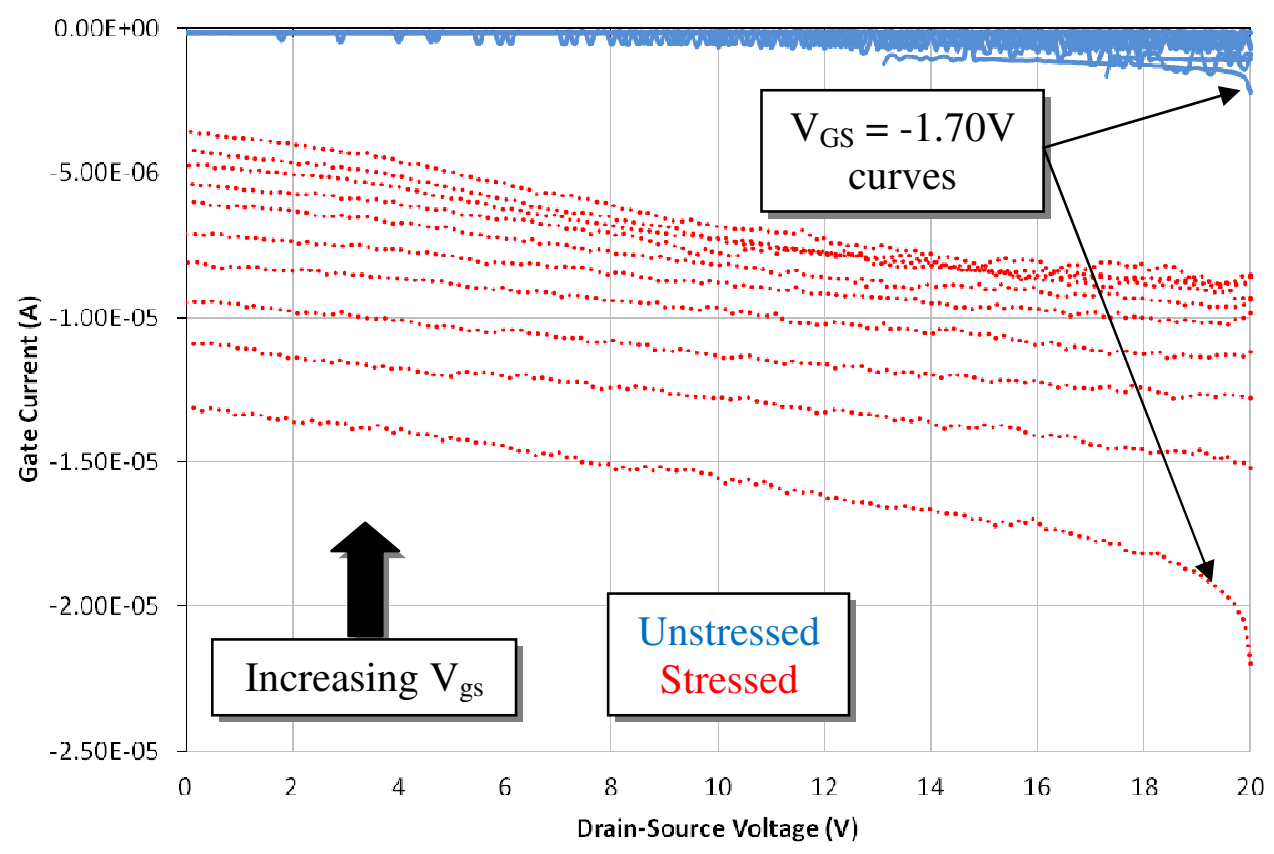

Figure 3.9 ? Gate current measurements before and after high symmetric electric field step-stress procedure.

A significant change in gate current was observed after performing a symmetric high-field step-stress experiment on each GaN-on-Si DUT (Figure 3.9). The increase in gate current seen in Figure 3.9 correlates to the increase in gate leakage current experienced during the high-field stress experiments (Figure 3.6 and Figure 3.7). As mentioned in Section 2.1, the GaN-on-Si HEMT DUTs possess Schottky gates made from Ni/Au. The Schottky gate is essentially a Schottky diode structure, formed by the metal-on-semiconductor junction between $\mathrm{Ni} / \mathrm{Au}$ and the $\mathrm{GaN}$ cap. The joining of the two materials develops what is known as a Schottky barrier height $\left(\phi_{\mathrm{B}}\right)$, which is the potential difference between the Fermi energy of the metal and the band edge where the majority carriers reside [27]. Figure 3.10 shows an example of Schottky diode band diagram at thermal equilibrium (no external voltage applied). As a voltage bias is applied 
to the device and an electric field is induced across the junction, $\phi_{\mathrm{B}}$ will lower respectively. The change in the barrier height can be found through Equation (3.1).

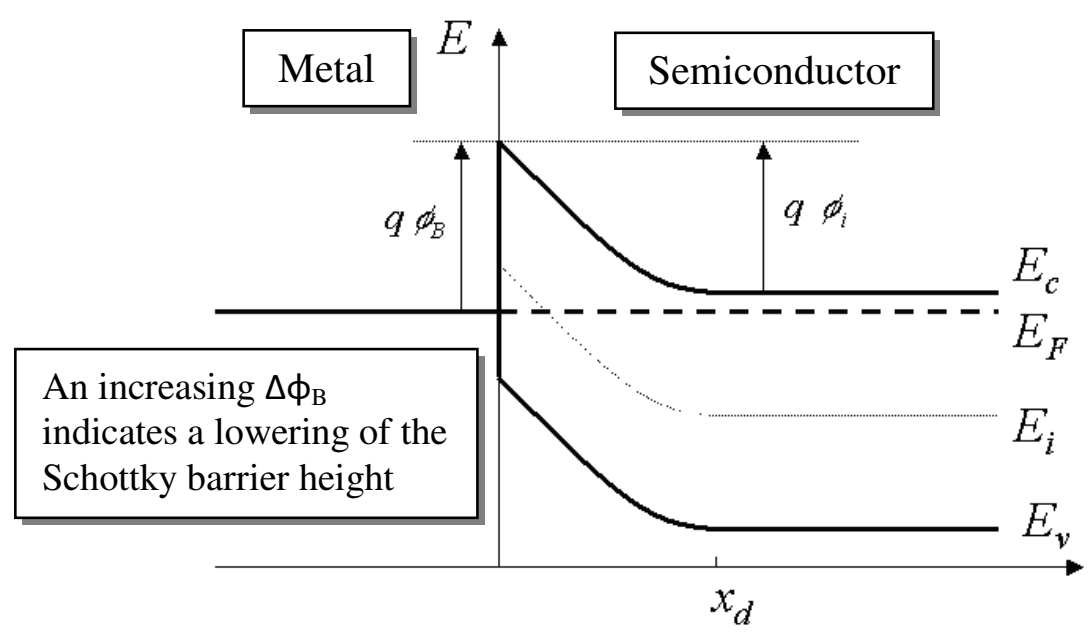

Figure 3.10 $\square$ Energy band diagram of a metal-semiconductor contact in thermal equilibrium [27].

$$
\Delta \phi_{B}=\sqrt{\frac{q E_{\max }}{4 \pi \varepsilon_{s}}}
$$

q: Elementary charge $\left(1.602 \times 10^{-19} \mathrm{C}\right)$

$\mathrm{E}_{\text {max }}$ : Electric field at the metal - semiconductor interface

$\varepsilon_{S}:$ Dielectric constant of the semiconductor

Because a high vertical electric field is induced at the gate of the HEMT devices during the symmetrical step-stress experiments, the Schottky barrier height of these devices is lowered substantially. As a result, gate leakage is enhanced due to a reduction in barrier potential. Work performed by Lo, et. al. revealed how GaN HEMTs with Ni/Au Schottky gate contacts (which is what the NPTB00004 possesses) experienced a 
reduction in $\phi_{\mathrm{B}}$ from $1.09 \mathrm{~V}$ to $0.66 \mathrm{~V}$, whereas devices with $\mathrm{Pt} / \mathrm{Ti} / \mathrm{Au}$ contacts experienced negligible changes from $1.23 \mathrm{~V}$ to $1.21 \mathrm{~V}$ [28].

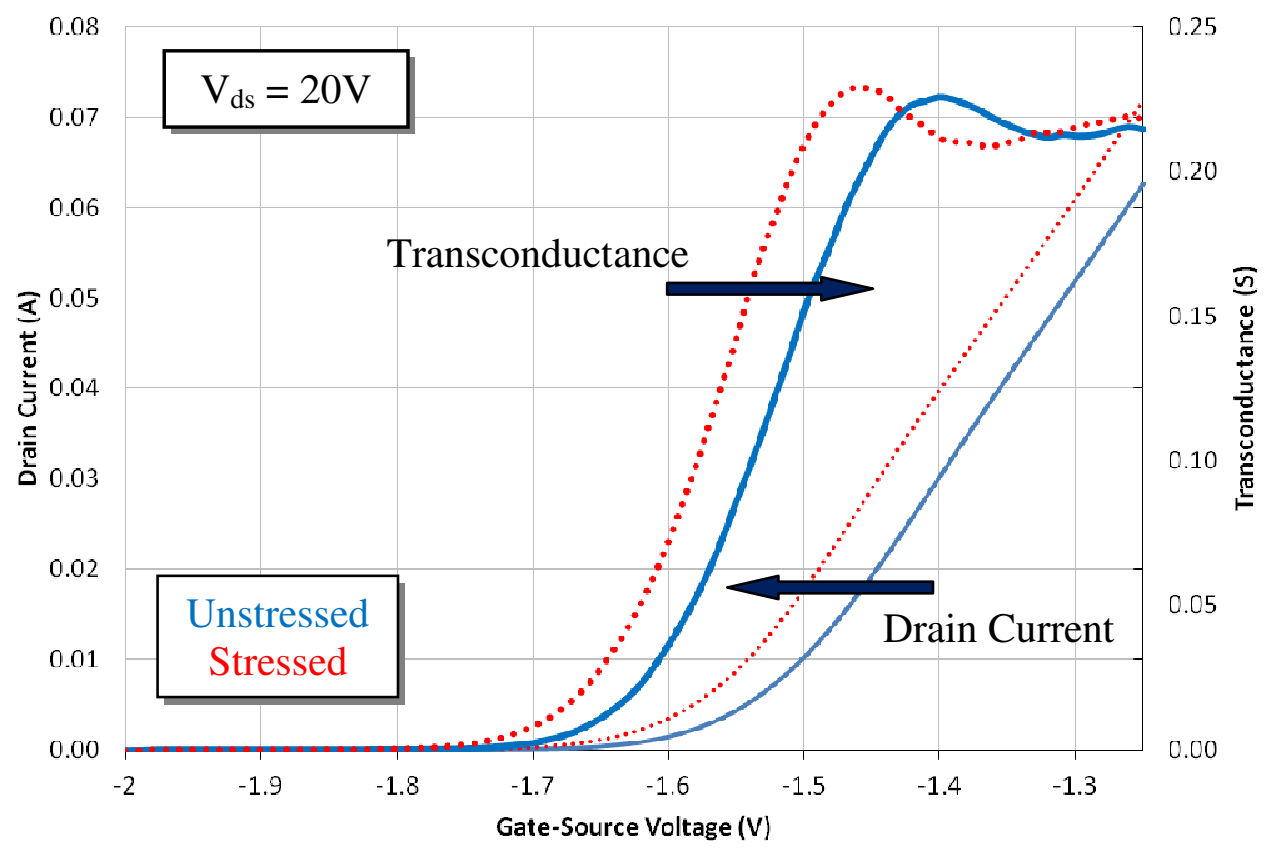

Figure 3.11 ? Transfer characteristics before and after high symmetric electric field step-stress procedure.

A negative threshold voltage shift was observed within each device after performing the step-stress experiment. The negative shift was determined to be an indication of electron detrapping events occurring within the AlGaN layer of the GaN-onSi HEMTs, resulting in a recovery of the 2DEG channel [24]. Before exposing each device to high electric fields in [24], the devices developed process-dependent defects during the growth process which in turn led to localized trap sites within the AlGaN barrier. The inherent electron traps within each fresh device then depleted the 2DEG region via electron trapping, resulting in a negative threshold voltage shift within the HEMTs (positive shifts will be covered later). Carbon tetrafluoride $\left(\mathrm{CF}_{4}\right)$ treatment experiments performed in [29] found that increased fluroine ion concentrations in the 
AlGaN layer localized near the 2DEG would deplete the channel of electrons and therefore induce positive threshold voltage shifts in their devices $\left(\mathrm{CF}_{4}\right.$ possesses a high electronegativity). Therefore, if a negative threshold voltage shift was observed in Figure 3.11, electron traps in the AlGaN barrier localized near the 2DEG may be detrapping and enhancing electron density in the channel.
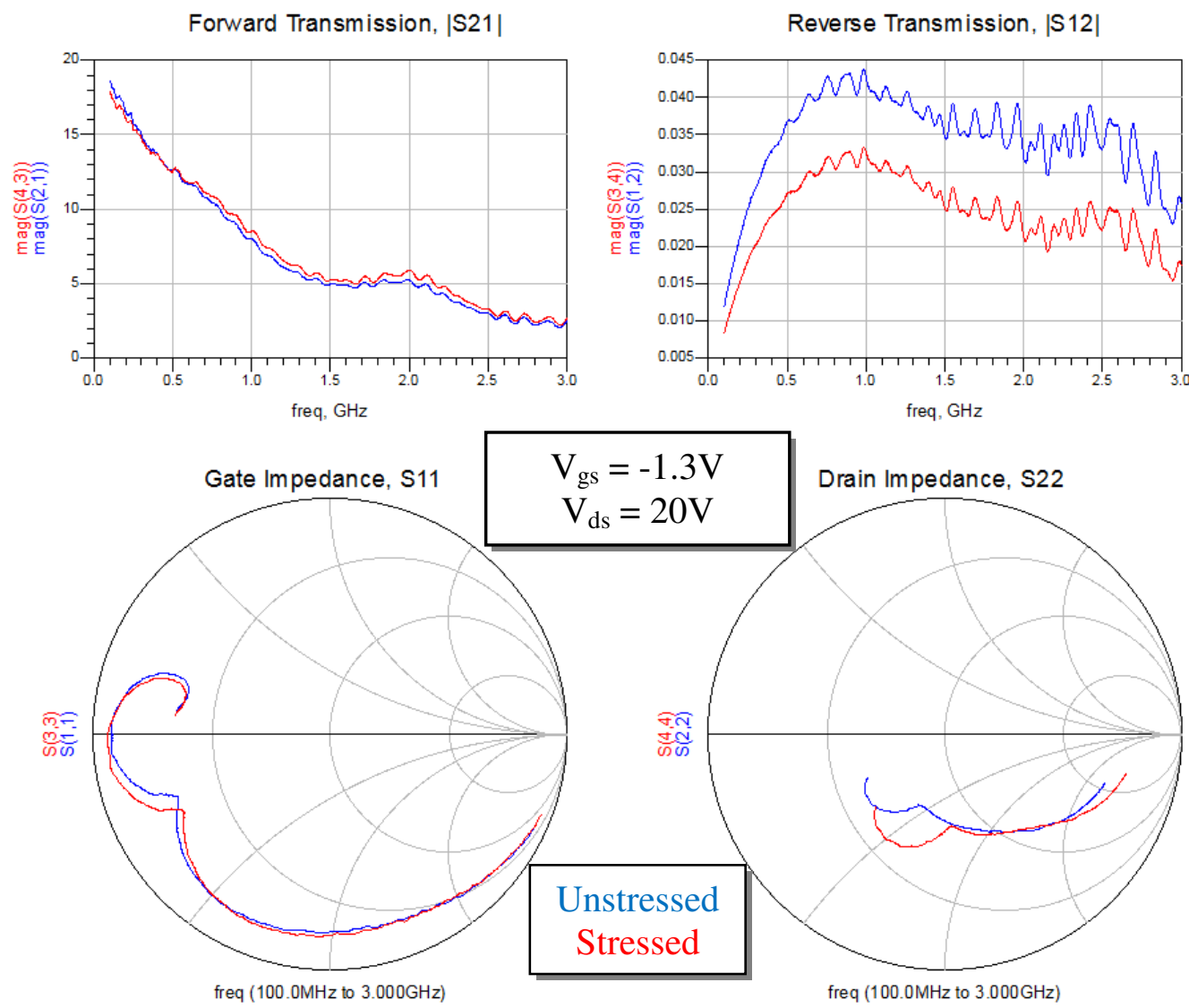

Figure 3.12 ? Measured S-parameters before and after high symmetric electric field stress.

The pre-stressed and post-stressed S-parameters are shown in Figure 3.12. An observable change in the small-signal parameters was measured, more specifically with the reverse transmission gain, $\left|\mathrm{S}_{12}\right|$. For microwave field-effect transistors (FETs), $\left|\mathrm{S}_{12}\right|$ is primarily controlled through the gate-drain capacitance, $\mathrm{C}_{\mathrm{gd}}$, which acts as the effective 
feedback in microwave amplifier designs [30]. As $C_{g d} \rightarrow 0,\left|S_{12}\right| \rightarrow 0$, which in turn means that the device becomes unilateral, or lacks feedback. Based upon the measurements in Figure 3.12, as the GaN-on-Si HEMT is exposed to high symmetric field stresses, the small-signal parameter $\mathrm{C}_{\mathrm{gd}}$ decreases and causes the device to become unilateral. A more detailed analysis on the DUT's small-signal characteristics can be seen in Chapter 6, where an equivalent small-signal model is developed to approximate the numerical changes in each of the small-signal parameters.

\subsubsection{Device Recovery Analysis after Symmetric Electric Field Stress}

An additional study was performed to observe whether or not the changes found within each DUT during the symmetrical electric field stress experiment persisted well after being performed. After stressing a pool of devices using the step-stress procedure, analyses similar to those performed in Subsection 3.2.2 were conducted. 24 hours after performing the stress procedures and taking initial measurements for each device, another round of measurements were made to assess the performance of the device after 24 hours of relaxation. The process was repeated every 24 hours until changes within each device ceased.

After relaxing for a period of 6 days, the drain current of each DUT showed no observable collapse or return to their corresponding current characteristics seen during the unstressed state. The increase and sustained drain current characteristics seen in Figure 3.13 further supported claims about electron trapping being prominent in the devices (process-dependent) prior to any exposure to high-field strain. 


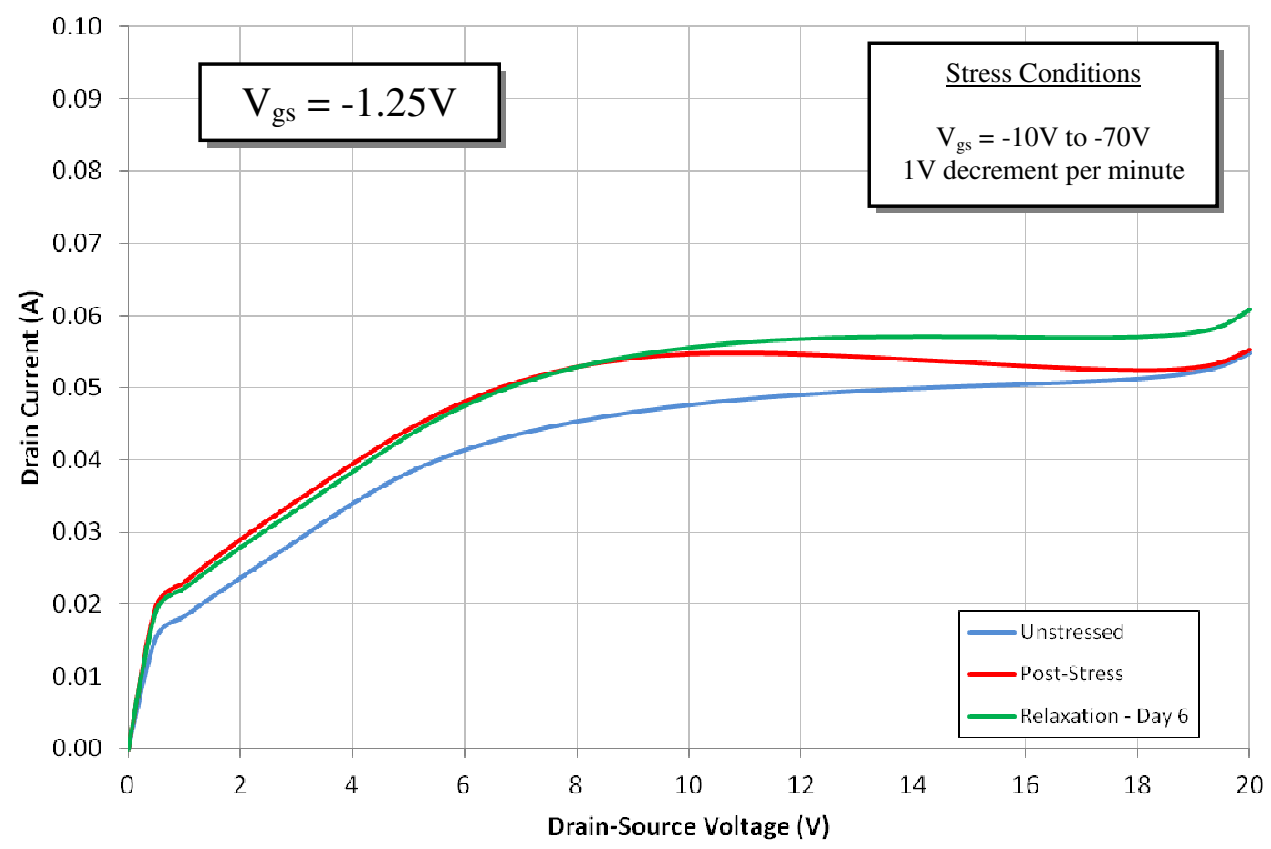

Figure 3.13 a Change in I-V characteristics over a relaxation period of 6 days.

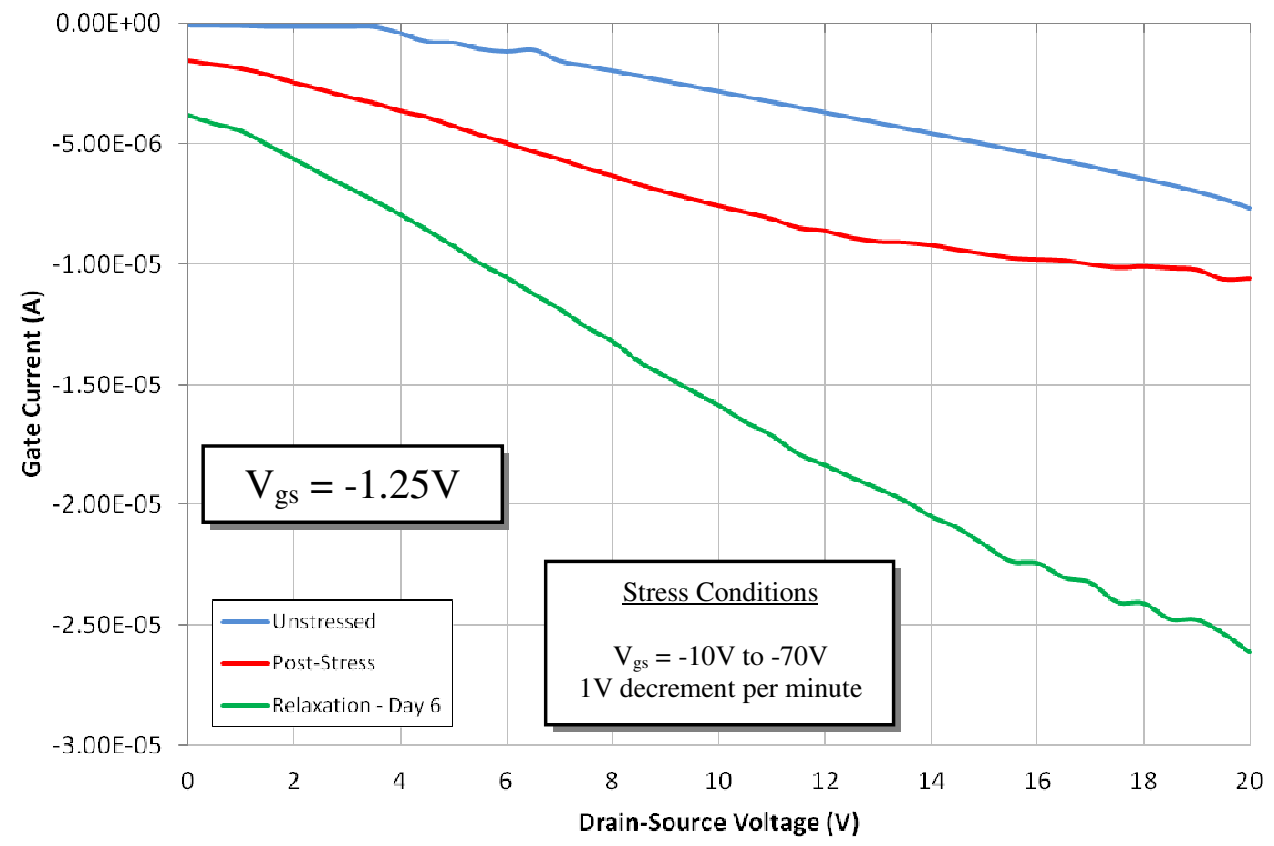

Figure 3.14 [ Observed gate current characteristics over a relaxation period of 6 days. 
Figure 3.14 shows a similar increase in gate current, as was seen in Figure 3.9. After relaxation over 6 days, however, the gate current continued to increase (green line). From Equation (3.1), it was found that high-electric fields within the HEMT devices reduce their Schottky barrier height, which in turn promoted gate leakage. Over the course of the 6-day relaxation period, none of the devices were exposed to high-field stress. While the phenomenon seen in Figure 3.14 cannot be attributed to high-field stress, the result may be due to a change in capacitance within the GaN cap. The change in capacitance would then affect the dielectric constant of the semiconductor and would alter the Schottky barrier height.

In reference to Equation (3.2) and holding all variables besides Q as constants, as the charge (Q) in a capacitor decreases, the dielectric constant $\left(\varepsilon_{\mathrm{r}}\right)$ will decrease proportionally [31]. Therefore, the Schottky barrier height of the GaN HEMTs may be inherently decreasing during the relaxation period due to possible electron detrapping events within the GaN cap, altering the charge stored within this region.

$$
\varepsilon_{r}=\varepsilon_{s}=C \frac{d}{\varepsilon_{o} A}=\frac{Q}{V} \frac{d}{\varepsilon_{o} A}
$$

Figure 3.15 shows a case where a negative threshold voltage shift was not observed directly after performing the symmetrical step-stress procedure. From Figure 3.11, a negative threshold voltage shift was expected for all GaN-on-Si devices after exposure to high symmetric fields. However, after sampling a larger group of devices, some of the stressed DUTs also experienced a positive threshold voltage shift. Work in [24] attributed the positive voltage shifts to lower electric fields resulting from 
stress voltages set well below the critical voltage. The lower fields were determined to further create trap states within the AlGaN barrier or GaN cap by changing their relative positions. Therefore, some of the stressed devices throughout the studies in this paper may not have experienced electron detrapping via the step-stress experiment. While the induced electric fields may not have promoted electron detrapping in some of the DUTs during the step-stress experiments, a negative voltage shift was found in all devices after the relaxation periods.

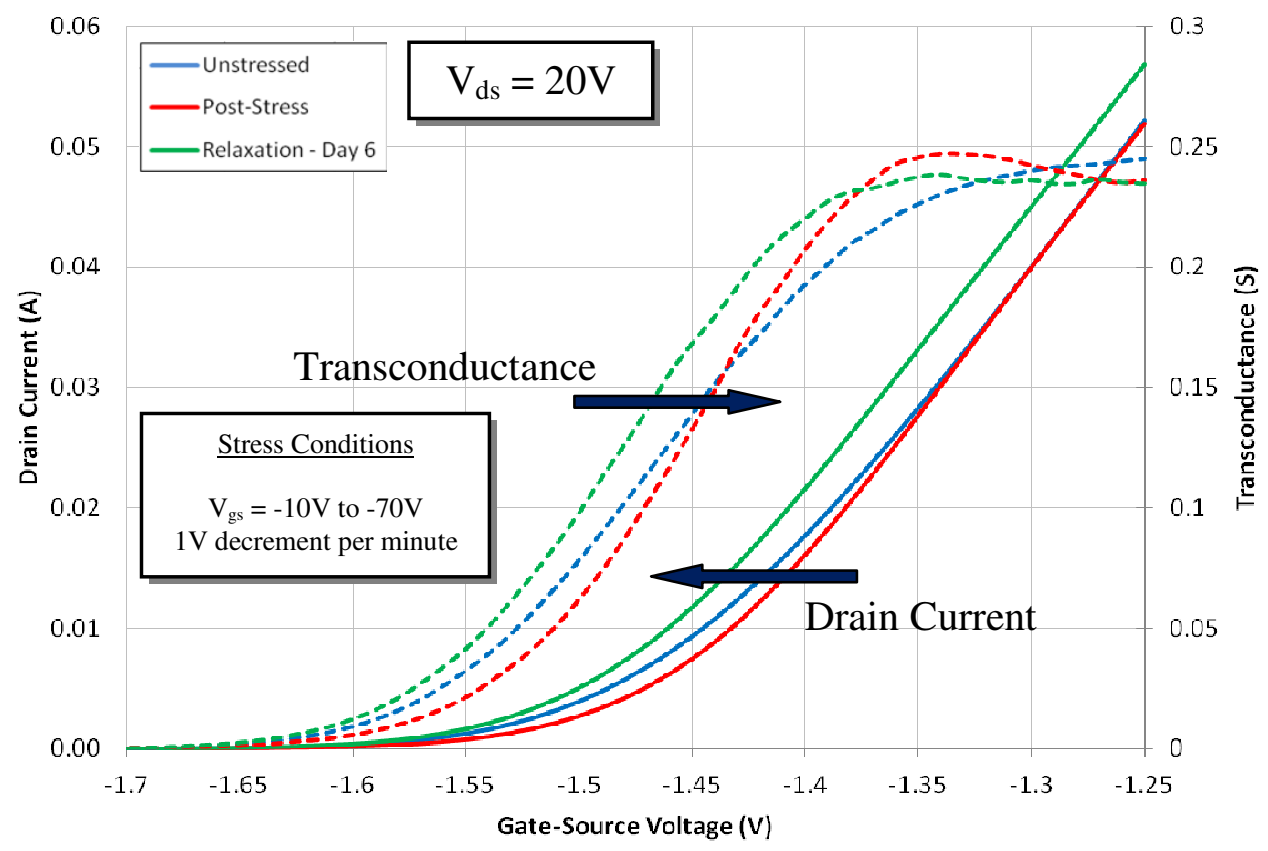

Figure 3.15 ? Observed transfer characteristics over a relaxation period of 6 days.

Minor alterations to the input impedance, output impedance, and forward transmission gain of each device were observed during the relaxation periods. The corresponding S-parameter relaxation measurements are shown in Figure 3.16. A noticeable recovery in each device's reverse transmission gain was measured. The 
recovery in $\left|S_{12}\right|$ after 6 days, however, was not complete. Further investigation of the $\mathrm{S}$ parameters is performed in Chapter 6.

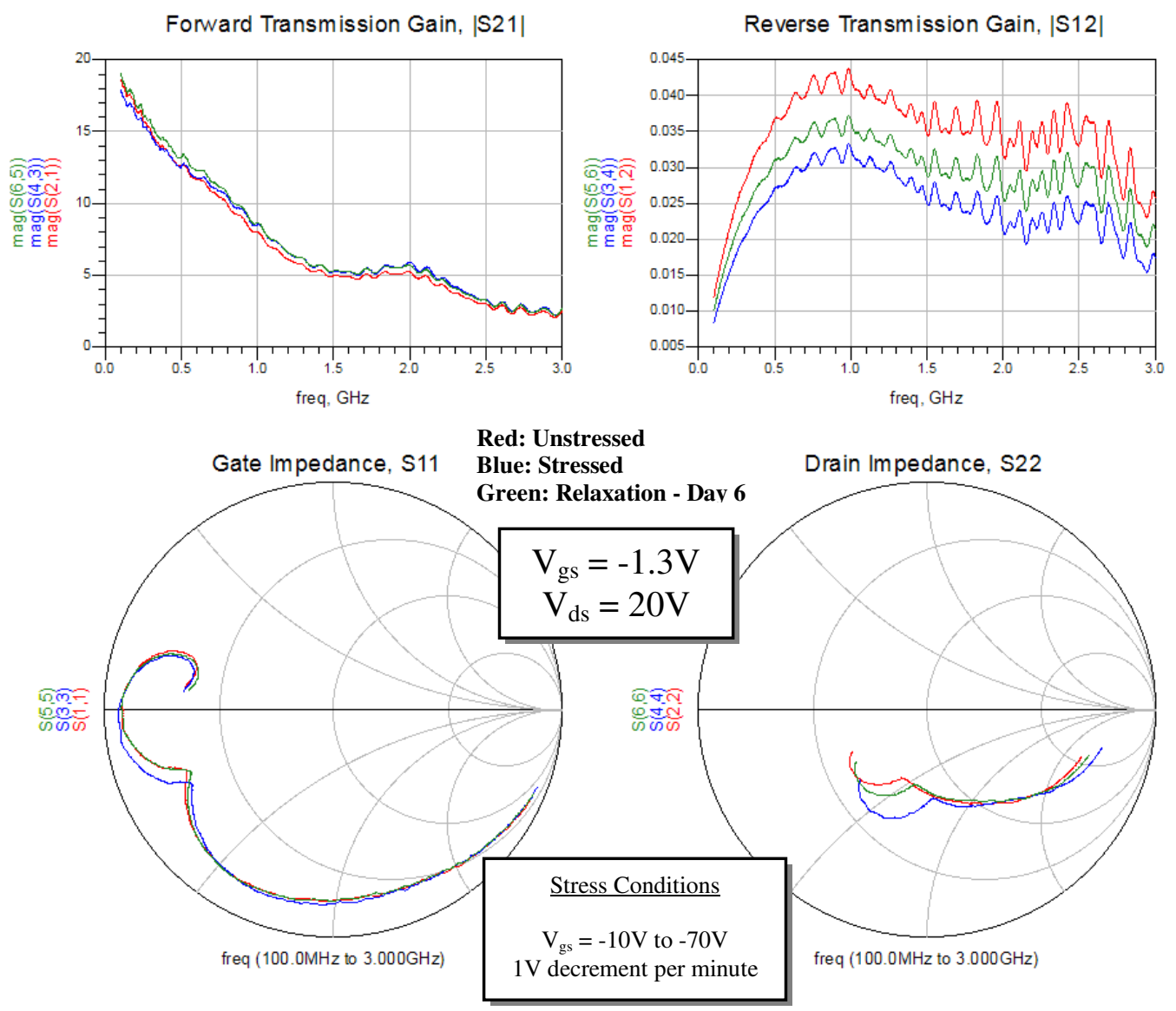

Figure 3.16 ? Change in S-parameters over a relaxation period of 6-days. 


\section{Experiment 2: Temperature Characterization}

\subsection{Effect of Temperature on General Device Performance}

\subsubsection{Setup}

A test structure was constructed to interface with an ILX Lightwave LDC-3744B, as seen in Figure 4.1 and Figure 4.2. The setup utilizes the same copper test structure as Figure 2.4 (pg. 15), but utilizes two thermoelectric coolers (TECs) to provide a symmetric distribution of heat onto the source-plane of the HEMT.

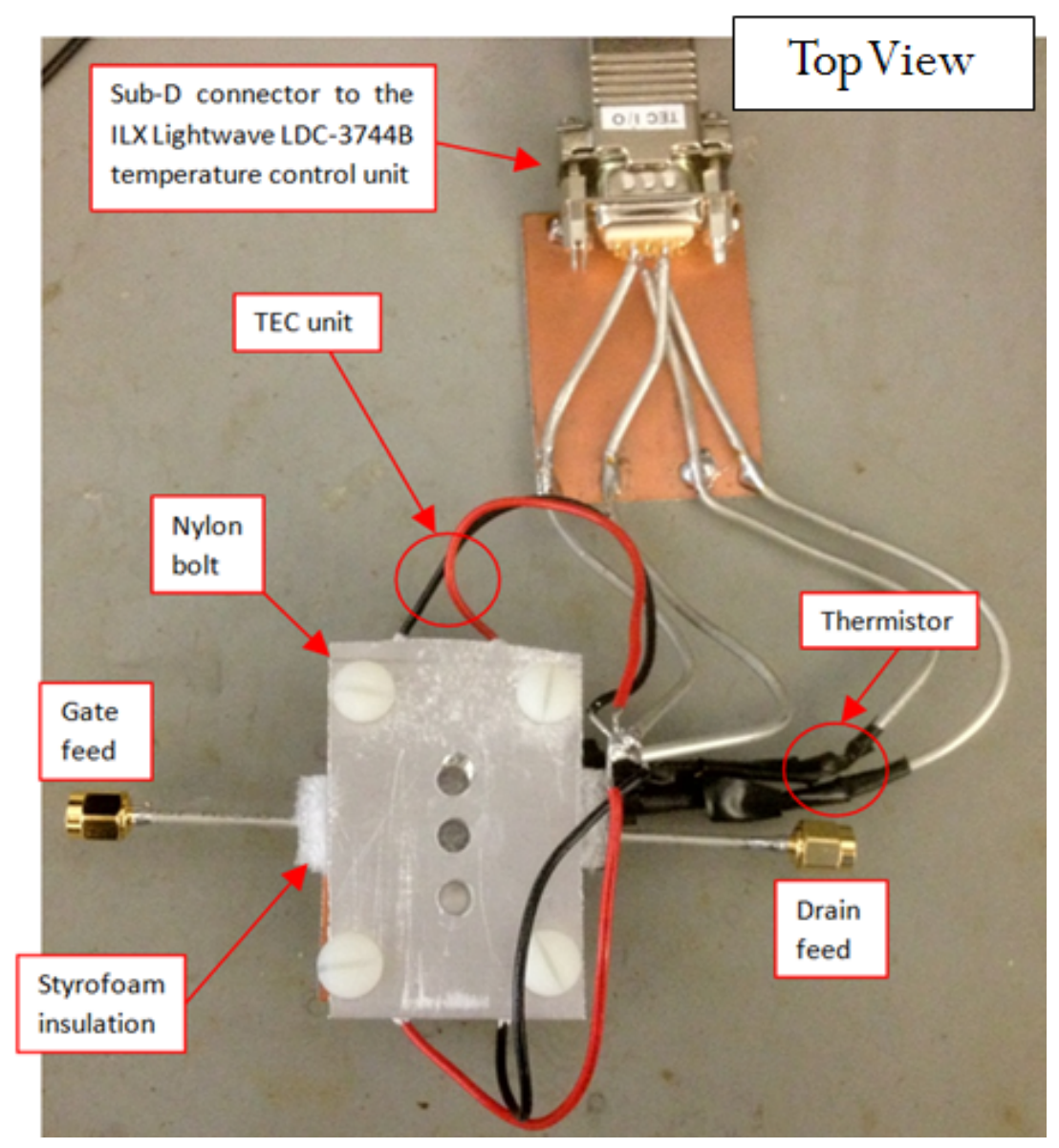

Figure 4.1 ? Top view of the test fixture designed for the thermal experiments. 
Because TECs develop a temperature gradient, it was necessary to use nylon bolts to provide high thermal resistance between the aluminum heat sink and the copper source-plane (back-plate). In many of the other experiments, metallic bolts were used to secure the aluminum heat sinks to the copper source-plane, which also promoted heat transfer between the source of the DUT and the heat sink. Therefore, it was necessary to prevent the transfer of heat between the two planes to better control the back-plate temperature.

\section{Side View}

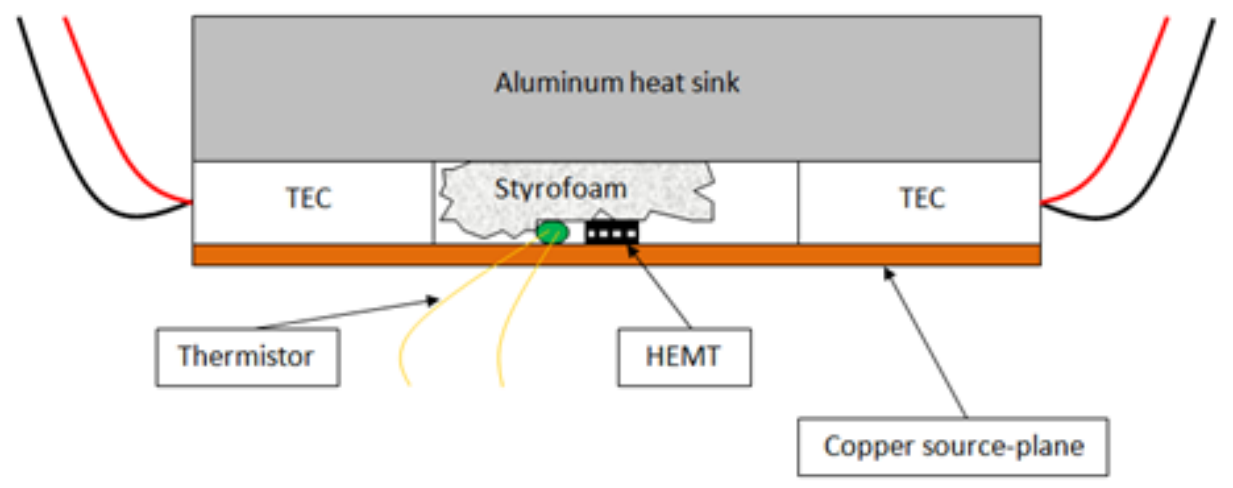

Figure 4.2 [ Side view concept drawing of the test fixture designed for the thermal experiments.

The original design did not incorporate the use of the styrofoam insulation between the heat sink and the thermistor. As a result, preliminary temperature readings from the ILX control unit did not provide accurate source-plane temperature measurements and instead would fluctuate between the heat sink temperature and the source-plane temperature (due to the temperature gradient). After providing thermal insulation, the readings increased in accuracy and the set point temperature for the source-plane was allowed to stabilize much faster. Thermal grease was also used to better conduct heat transfer between the back-plate and the thermistor element. 
DC and RF tests were performed in this section under three back-plate temperature conditions: $27^{\circ} \mathrm{C}, 50^{\circ} \mathrm{C}$, and $100^{\circ} \mathrm{C}$. Once the back-plate temperature of the DUT stabilized to the desired temperature, I-V characteristics, transfer characteristics, and S-parameters were measured (refer to Subsections 2.2.1, 2.2.2, and 2.2.3 for excitation settings). After each DUT was stressed to $100^{\circ} \mathrm{C}$, the devices were allowed to relax over a 24 hour span. After relaxation, the characteristics were reevaluated at $27^{\circ} \mathrm{C}$ and compared to the measurement results at $27^{\circ} \mathrm{C}$ from the previous day to determine if temperature-dependent degradation had occurred.

To determine the junction temperature for each device under a given back-plate temperature and voltage biasing condition, Equation (4.1) was used below [32]. $\mathrm{P}_{\text {DISS }}$ in Equation (4.1) is the drain quiescent current multiplied by the drain-source voltage. The same equation can be used in the experiments of Section 4.2. For the devices used in this study, $\theta_{\mathrm{JC}}$ was given in [13] to be $23^{\circ} \mathrm{C} / \mathrm{W}$.

$$
\mathrm{T}_{\mathrm{J}}=T_{\text {Baseplate }}+\theta_{J C} * P_{D I S S}
$$

$$
\begin{aligned}
& \mathrm{T}_{\mathrm{J}} \text { : Junction temperature }\left({ }^{\circ} \mathrm{C}\right) \\
& \mathrm{T}_{\text {baseplate }} \text { : Base }- \text { plate temperature }\left({ }^{\circ} \mathrm{C}\right) \\
& \theta_{\mathrm{JC}} \text { : Thermal resistance between the junction and case }\left({ }^{\circ} \mathrm{C} / \mathrm{W}\right) \\
& P_{\text {DISS }} \text { : Dissipated power }(\mathrm{W})
\end{aligned}
$$

\subsubsection{Results and Analyses}

Figure 4.3 shows the resulting $\mathrm{I}-\mathrm{V}$ characteristics at $\mathrm{V}_{\mathrm{gs}}=-1.25$ after exposure to back-plate temperatures of $27^{\circ} \mathrm{C}, 50^{\circ} \mathrm{C}$, and $100^{\circ} \mathrm{C}$ while Figure 4.4 shows a comparison between I-V curves taken at room temperature before thermal stress and 
24 hours after high thermal stress. From Figure 4.3, it was observed that the overall drain current of the device becomes suppressed with increasing back-plate temperatures while Figure 4.4 shows a negligible reduction in overall drain current after exposure to high thermal stress. The junction temperature of the DUT in Figure 4.3 at $\mathrm{V}_{\mathrm{gs}}=-1.25 \mathrm{~V}$ and $\mathrm{V}_{\mathrm{ds}}=10 \mathrm{~V}$ is shown in Table 4.1. The junction temperature of the same DUT at $\mathrm{V}_{\mathrm{gs}}=-1.25 \mathrm{~V}$ and $\mathrm{V}_{\mathrm{ds}}=15 \mathrm{~V}$, and $\mathrm{V}_{\mathrm{gs}}=-1.25 \mathrm{~V}$ and $\mathrm{V}_{\mathrm{ds}}=20 \mathrm{~V}$ is shown in Table 4.2 and Table 4.3, respectively. Under all conditions, it can be seen that the power dissipated in the device decreases with increasing base-plate temperature, and the temperature difference between the base-plate and the junction becomes smaller.

Table 4.1 ? DUT junction temperature at $\mathrm{V}_{\mathrm{gs}}=-1.25 \mathrm{~V}$ and $\mathrm{V}_{\mathrm{ds}}=10 \mathrm{~V}$.

\begin{tabular}{|c|c|c|c|c|}
\hline $\begin{array}{c}\text { Base-plate Temperature, } \\
\boldsymbol{T}_{\text {Baseplate }}\left({ }^{\circ} \boldsymbol{C}\right)\end{array}$ & $\begin{array}{c}\text { Drain Current (mA) } \\
\boldsymbol{V}_{g s}=\mathbf{- 1 . 2 5}, \boldsymbol{V}_{d s}=\mathbf{1 0 V}\end{array}$ & $\begin{array}{c}\text { Dissipated } \\
\text { Power, } \boldsymbol{P}_{\text {DISS }}(\boldsymbol{W})\end{array}$ & $\begin{array}{c}\text { Junction } \\
\text { Temperature, } \boldsymbol{T}_{\boldsymbol{J}}\left({ }^{\circ} \boldsymbol{C}\right)\end{array}$ & $\begin{array}{c}\Delta \boldsymbol{\Delta T}\left({ }^{\circ} \boldsymbol{C}\right) \\
\boldsymbol{T}_{\text {Baspplate }}-\boldsymbol{T}_{\boldsymbol{J}}\end{array}$ \\
\hline 27 & 89 & 0.890 & 47.47 & 20.47 \\
\hline 50 & 78 & 0.780 & 67.94 & 19.94 \\
\hline 100 & 52 & 0.520 & 111.96 & 11.96 \\
\hline
\end{tabular}

Table 4.2 ? DUT junction temperature at $\mathrm{V}_{\mathrm{gs}}=-1.25 \mathrm{~V}$ and $\mathrm{V}_{\mathrm{ds}}=15 \mathrm{~V}$.

\begin{tabular}{|c|c|c|c|c|}
\hline $\begin{array}{c}\text { Base-plate Temperature, } \\
\boldsymbol{T}_{\text {Basplate }}\left({ }^{\circ} \boldsymbol{C}\right)\end{array}$ & $\begin{array}{c}\text { Drain Current }(\boldsymbol{m A}) \\
\boldsymbol{V}_{\boldsymbol{g s}}=\mathbf{- 1 . 2 5}, \boldsymbol{V}_{\boldsymbol{d s}}=\mathbf{1 5 V}\end{array}$ & $\begin{array}{c}\text { Dissipated } \\
\text { Power, } \boldsymbol{P}_{\text {DISS }}(\boldsymbol{W})\end{array}$ & $\begin{array}{c}\text { Junction } \\
\text { Temperature, } \boldsymbol{T}_{\boldsymbol{J}}\left({ }^{\boldsymbol{C}} \boldsymbol{C}\right)\end{array}$ & $\begin{array}{c}\Delta \boldsymbol{T}\left({ }^{\circ} \boldsymbol{C}\right) \\
\boldsymbol{T}_{\text {Baseplate }}-\boldsymbol{T}_{\boldsymbol{J}}\end{array}$ \\
\hline 27 & 88 & 1.32 & 57.36 & 30.36 \\
\hline 50 & 79 & 1.19 & 77.37 & 27.37 \\
\hline 100 & 56 & 0.84 & 119.32 & 19.32 \\
\hline
\end{tabular}


Table 4.3 ? DUT junction temperature at $\mathrm{V}_{\mathrm{gs}}=-1.25 \mathrm{~V}$ and $\mathrm{V}_{\mathrm{ds}}=20 \mathrm{~V}$.

\begin{tabular}{|c|c|c|c|c|}
\hline $\begin{array}{c}\text { Base-plate Temperature, } \\
\boldsymbol{T}_{\text {Baseplate }}\left({ }^{\circ} \boldsymbol{C}\right)\end{array}$ & $\begin{array}{c}\text { Drain Current }(\boldsymbol{m A}) \\
\boldsymbol{V}_{g s}=\mathbf{- 1 . 2 5}, \boldsymbol{V}_{\boldsymbol{d s}}=\mathbf{2 0 V}\end{array}$ & $\begin{array}{c}\text { Dissipated } \\
\text { Power, } \boldsymbol{P}_{\text {DISS }}(\boldsymbol{W})\end{array}$ & $\begin{array}{c}\text { Junction } \\
\text { Temperature, } \boldsymbol{T}_{\boldsymbol{J}}\left({ }^{\circ} \boldsymbol{C}\right)\end{array}$ & $\begin{array}{c}\Delta \boldsymbol{\Delta T}\left({ }^{\circ} \boldsymbol{C}\right) \\
\boldsymbol{T}_{\text {Baseplate }}-\boldsymbol{T}_{\boldsymbol{J}}\end{array}$ \\
\hline 27 & 91 & 1.82 & 68.86 & 41.86 \\
\hline 50 & 81 & 1.62 & 87.26 & 37.26 \\
\hline 100 & 63 & 1.26 & 128.98 & 28.98 \\
\hline
\end{tabular}

Experimental results from [33], [34], and [35] also revealed a similar reduction in drain current with increasing back-plate temperatures. Aside from possessing a 16-17\% lattice mismatch between $\mathrm{Si}$ and $\mathrm{GaN}$, a 54-56\% thermal mismatch also exists, which promotes native traps within the device [17], [25]. The suppression in drain current was determined to be a direct result of reduced electron mobility and density within the 2DEG as a result of increased vibrational energy of the crystal lattice, and also from trapassisted tunneling (TAT) effects seen in the AlGaN barrier. TAT effects show exponential temperature dependence and become more relevant at elevated temperature ranges [33]. 


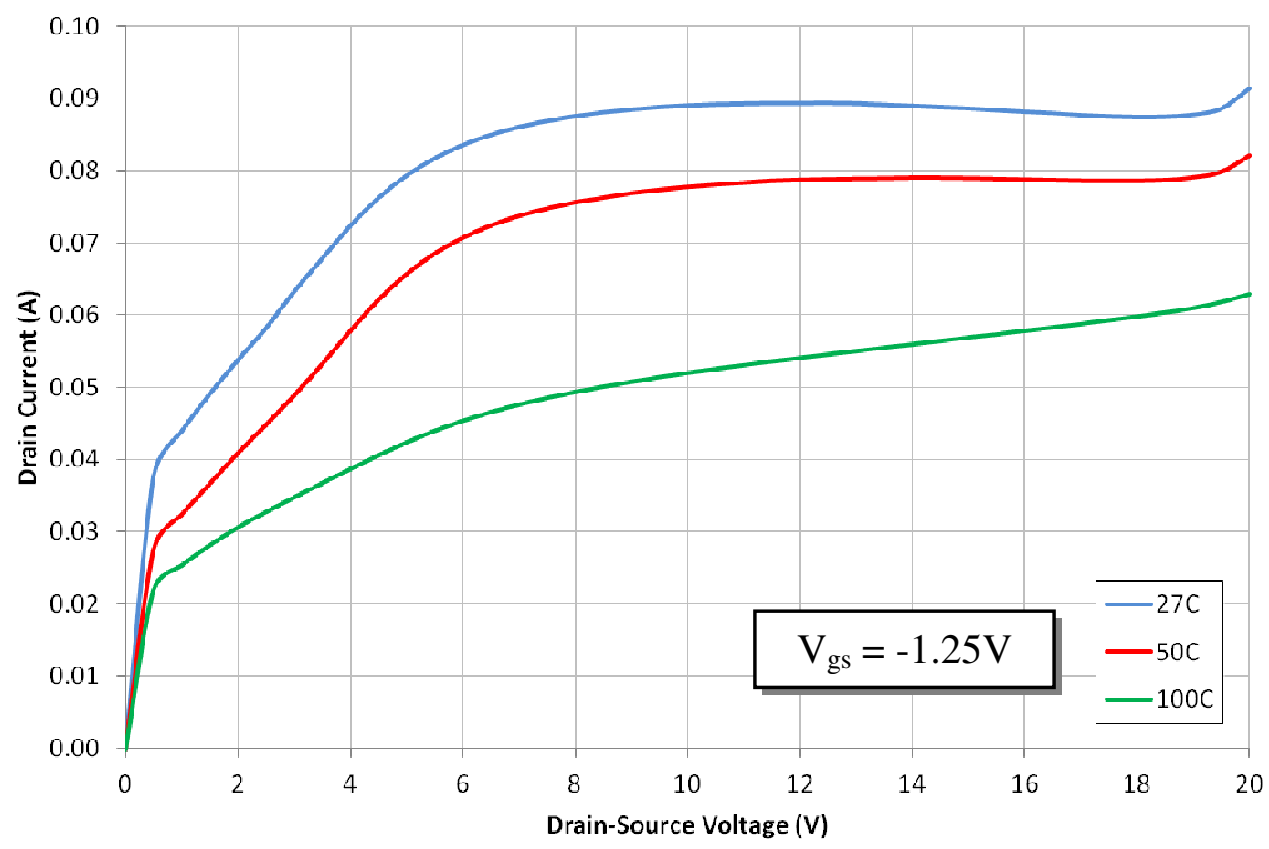

Figure 4.3 $\square \mathrm{I}-\mathrm{V}$ characteristics at $27^{\circ} \mathrm{C}, 50^{\circ} \mathrm{C}$, and $100^{\circ} \mathrm{C}$.

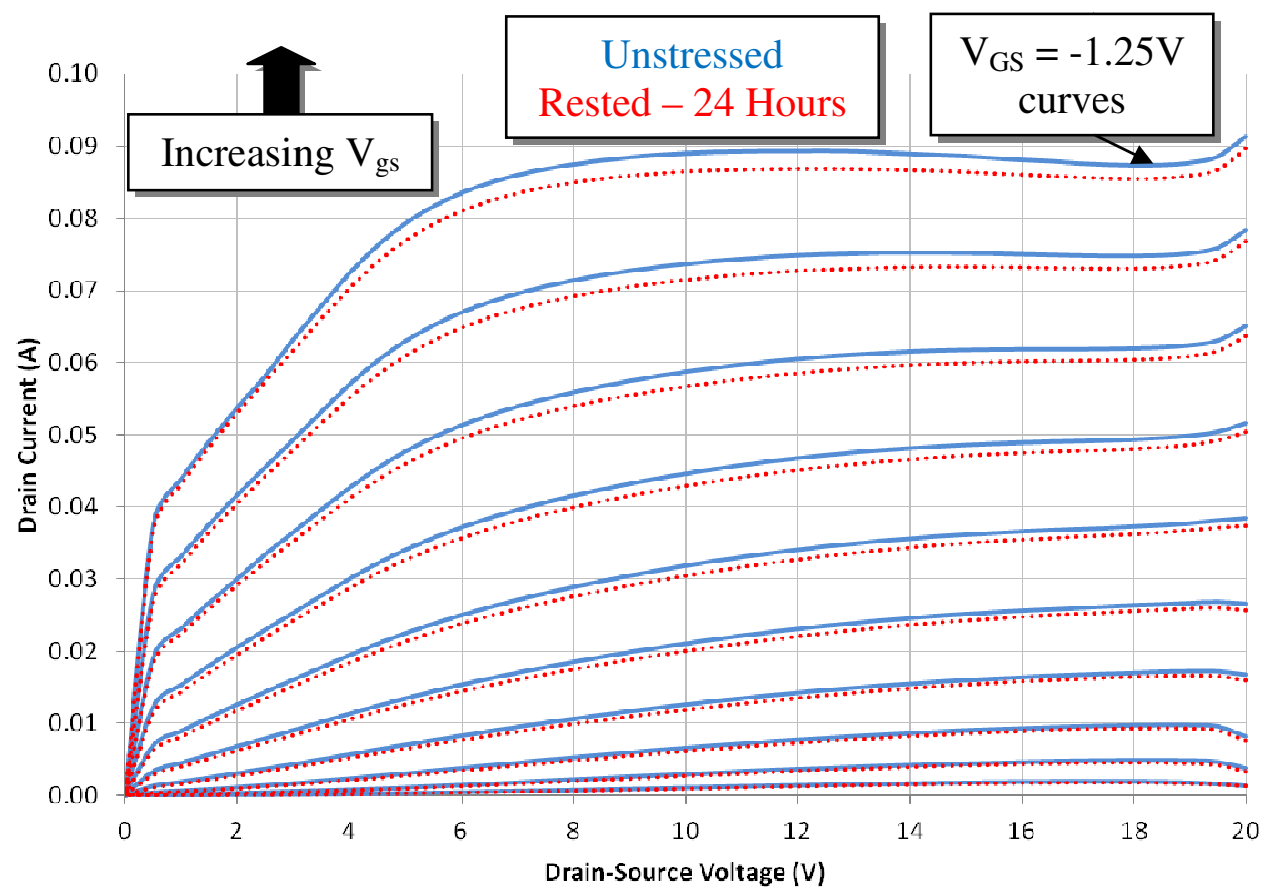

Figure 4.4 ? I-V characteristics comparison between a DUT at $27^{\circ} \mathrm{C}$ and at $27^{\circ} \mathrm{C} 24$ hours after thermal exposure at $100^{\circ} \mathrm{C}$. 
The TAT phenomenon also directly affects the gate leakage characteristics of the GaN-on-Si HEMTs. As seen in Figure 4.5, an increase in gate current is observed as the back-plate temperature of the representative DUT is increased. Trapped electrons in the $\mathrm{AlGaN}$ barrier are provided with the necessary energy to escape trap formations and tunnel through the AlGaN barrier as a direct result of increased temperatures, which in turn depletes the 2DEG and enhances gate leakage current [33]. The results seen in Figure 4.5 and the increased electron trapping behavior in the AlGaN barrier further support the current collapse observations seen in Figure 4.3.

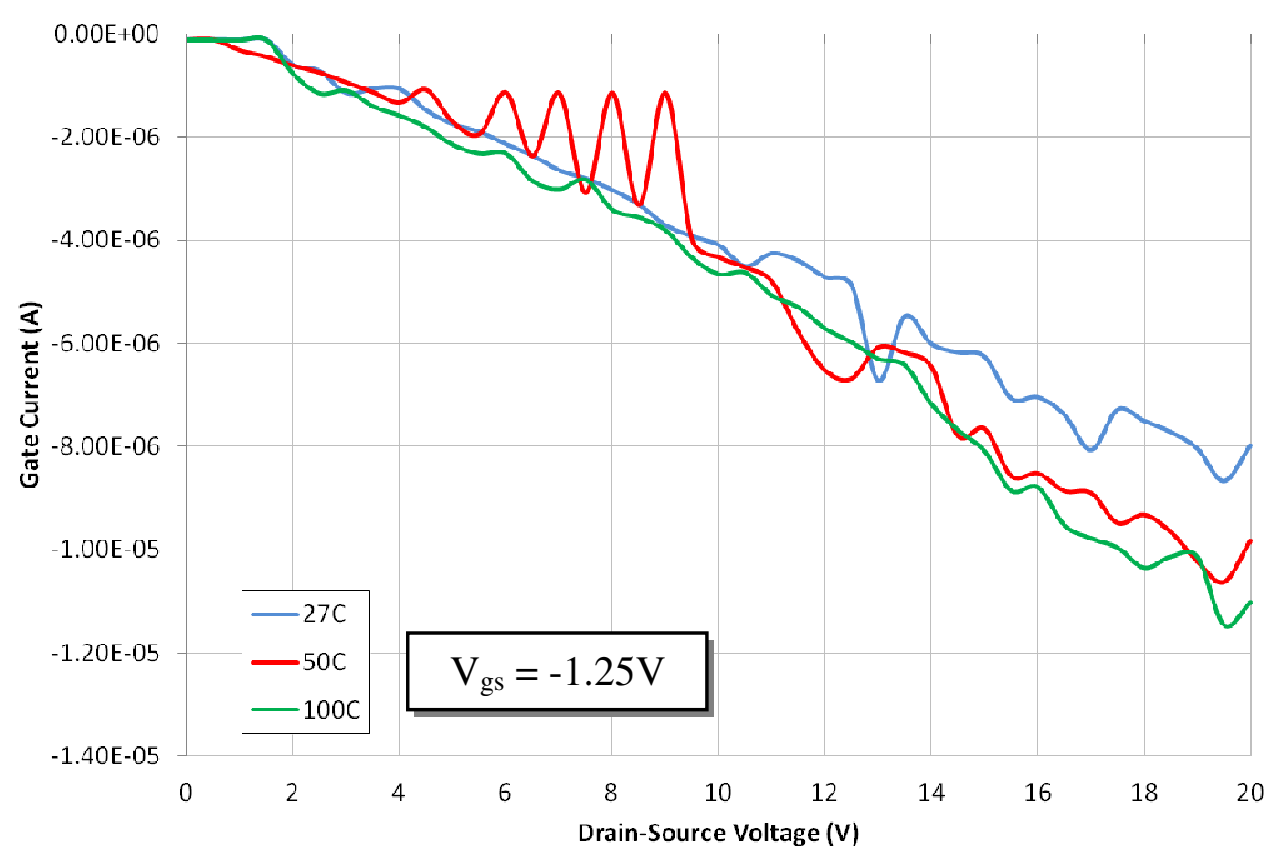

Figure 4.5 a Gate current characteristics at $27^{\circ} \mathrm{C}, 50^{\circ} \mathrm{C}$, and $100^{\circ} \mathrm{C}$.

Gate current measurements after 24 hours of relaxation are shown in Figure 4.6. The measurements show a prominent reduction in gate current as a result of the relaxation at room temperature. A reduction in gate current would seem to indicate an increased recovery of electrons in the 2DEG, but the results of Figure 4.4 may suggest 
otherwise. The work done by del Alamo and Joh in [10] shows that when the trap states in the $\mathrm{AlGaN}$ are filled with electrons, the gate leakage path is suppressed and the gate leakage current reduces as a result. The filled electron states also prevent the recovery of the 2DEG, which in turn suppresses the drain current of the GaN-on-Si HEMTs.

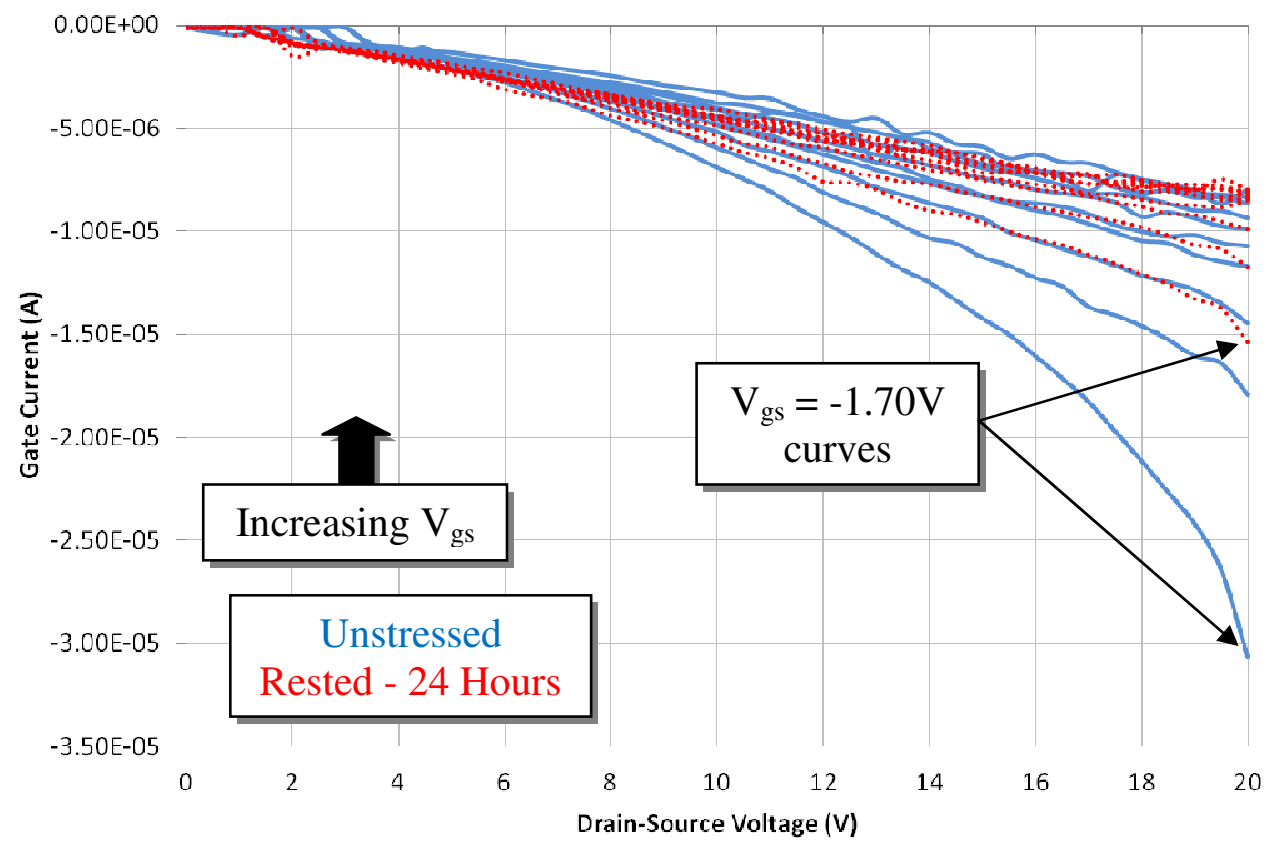

Figure 4.6 ? Gate current characteristics comparison between a DUT at $27^{\circ} \mathrm{C}$ and at $27^{\circ} \mathrm{C} 24$ hours after thermal exposure at $100^{\circ} \mathrm{C}$.

Increased back-plate temperatures also directly affected each DUT's transconductance and threshold voltage. As temperature increased, transconductance decreased and a positive threshold voltage-shift was observed (Figure 4.7), which coincides with the data presented in Table 4.1 through Table 4.3. The reduction in transconductance and positive-shift in threshold voltage was also determined to be a result of decreased electron density in the 2DEG [33]. Allowing the DUTs to relax for 24 hours resulted in a full recovery of the devices' transconductance characteristics and threshold voltage (Figure 4.8). 


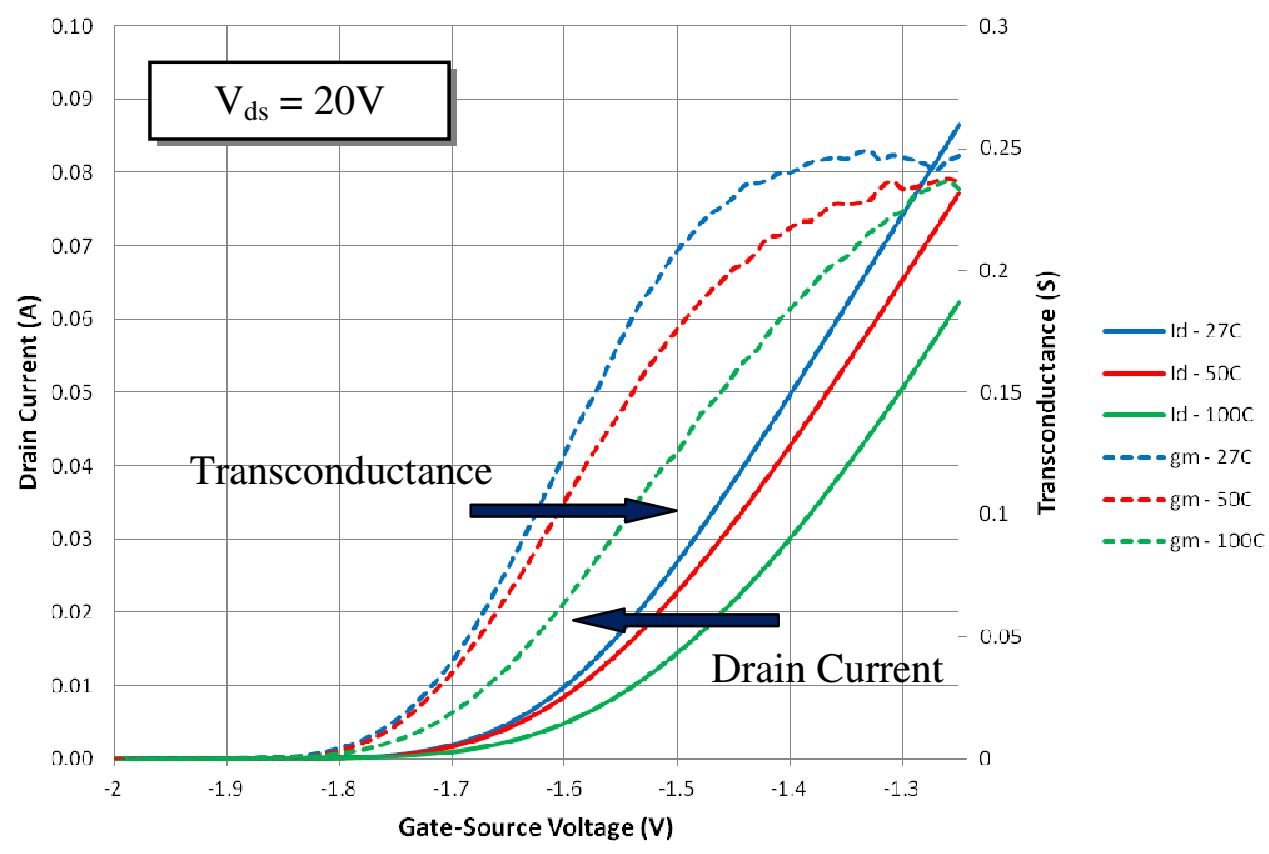

Figure 4.7 国 Transfer characteristics at $27^{\circ} \mathrm{C}, 50^{\circ} \mathrm{C}$, and $100^{\circ} \mathrm{C}$.

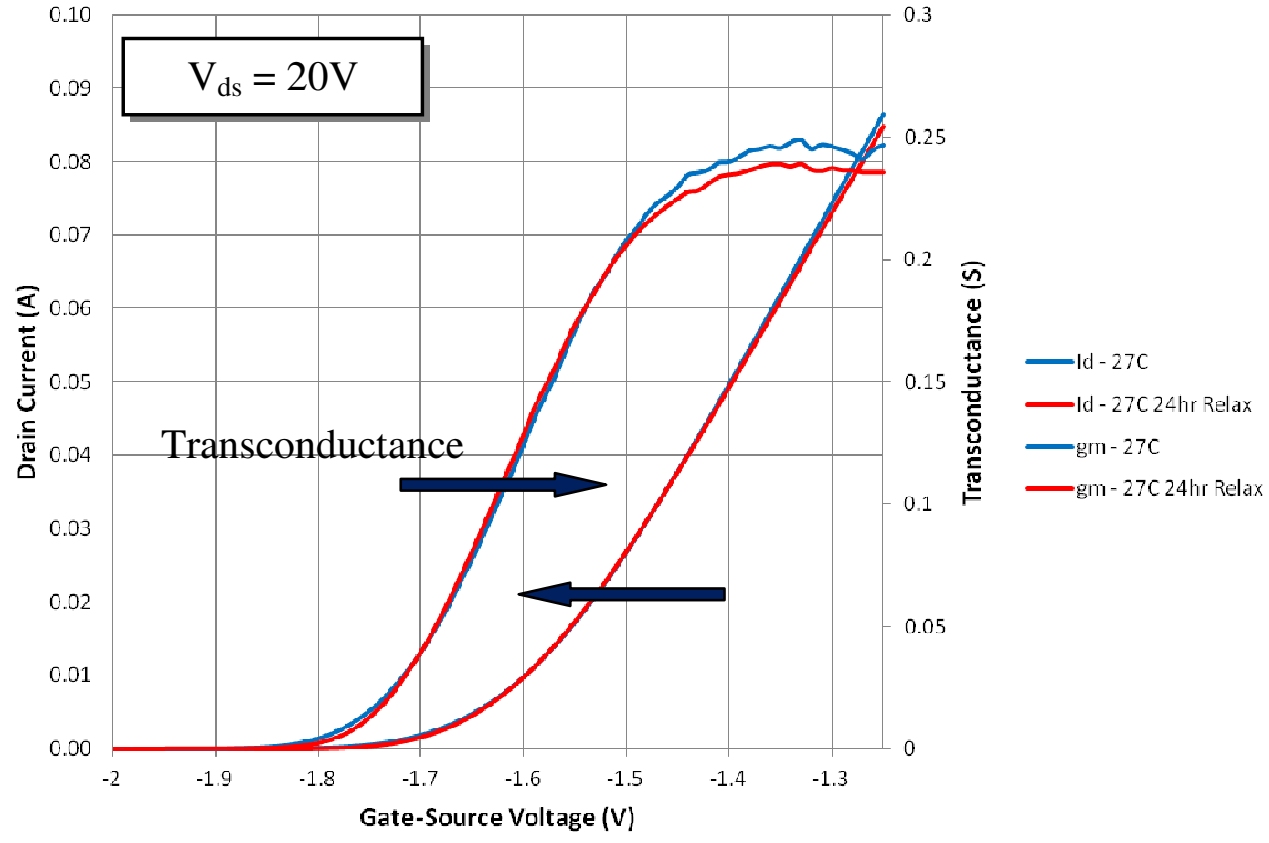

Figure 4.8 9 Transfer characteristics comparison between a DUT at $27^{\circ} \mathrm{C}$ and at $27^{\circ} \mathrm{C} 24$ hours after thermal exposure at $100^{\circ} \mathrm{C}$. 

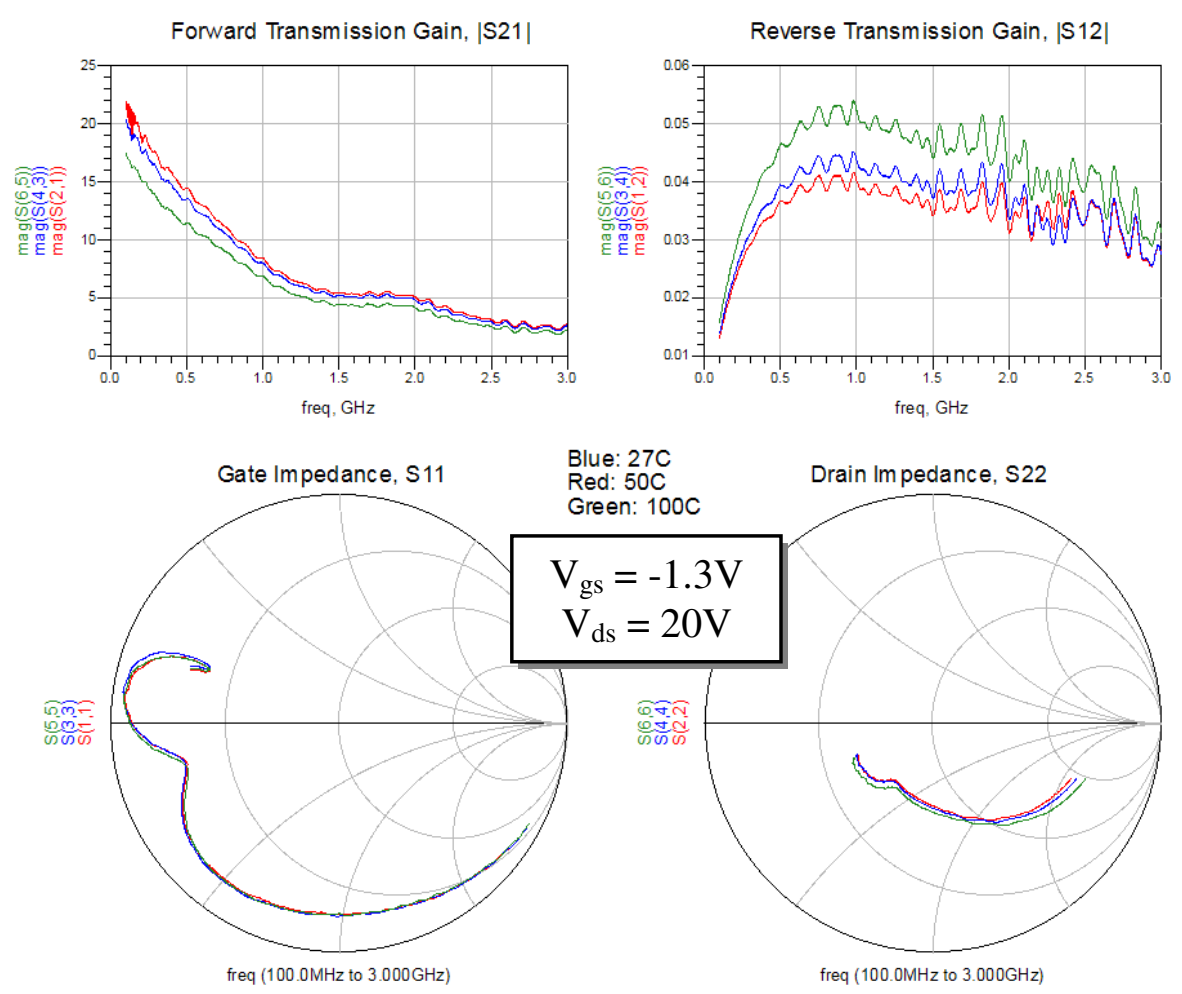

Figure 4.9 ? S-parameter measurements at $27^{\circ} \mathrm{C}, 50^{\circ} \mathrm{C}$, and $100^{\circ} \mathrm{C}$.

A reduction in low frequency gain was observed in the forward transmission gain $\left(\left|S_{21}\right|\right)$ measurements and an overall increase in reverse transmission gain $\left(\left|S_{12}\right|\right)$ was observed with increasing temperature (Figure 4.9). No significant changes to the input and output impedances were observed during the thermal stress tests. As was determined in Subsection 3.2.2, the reverse transmission gain is correlated to the gate-drain capacitance, $\mathrm{C}_{\mathrm{gd}}$, where an increase in $\left|\mathrm{S}_{12}\right|$ direct translates to an increase in $\mathrm{C}_{\mathrm{gd}}$. Smallsignal experiments performed in [36] found that $\mathrm{C}_{\mathrm{gd}}$ varied between $0.11 \% /{ }^{\circ} \mathrm{C}$ to $0.14 \% /{ }^{\circ} \mathrm{C}$ and that an increase in feedback capacitance reduced the overall gain and efficiency of their devices (Miller Effect). The reduction in $\left|S_{21}\right|$ also correlates to the reduction in transconductance seen in Figure 4.7 since forward transmission gain is 
proportional to transconductance. After 24 hours of relaxation, the S-parameters recovered fully to their nominal values, as seen in Figure 4.10.
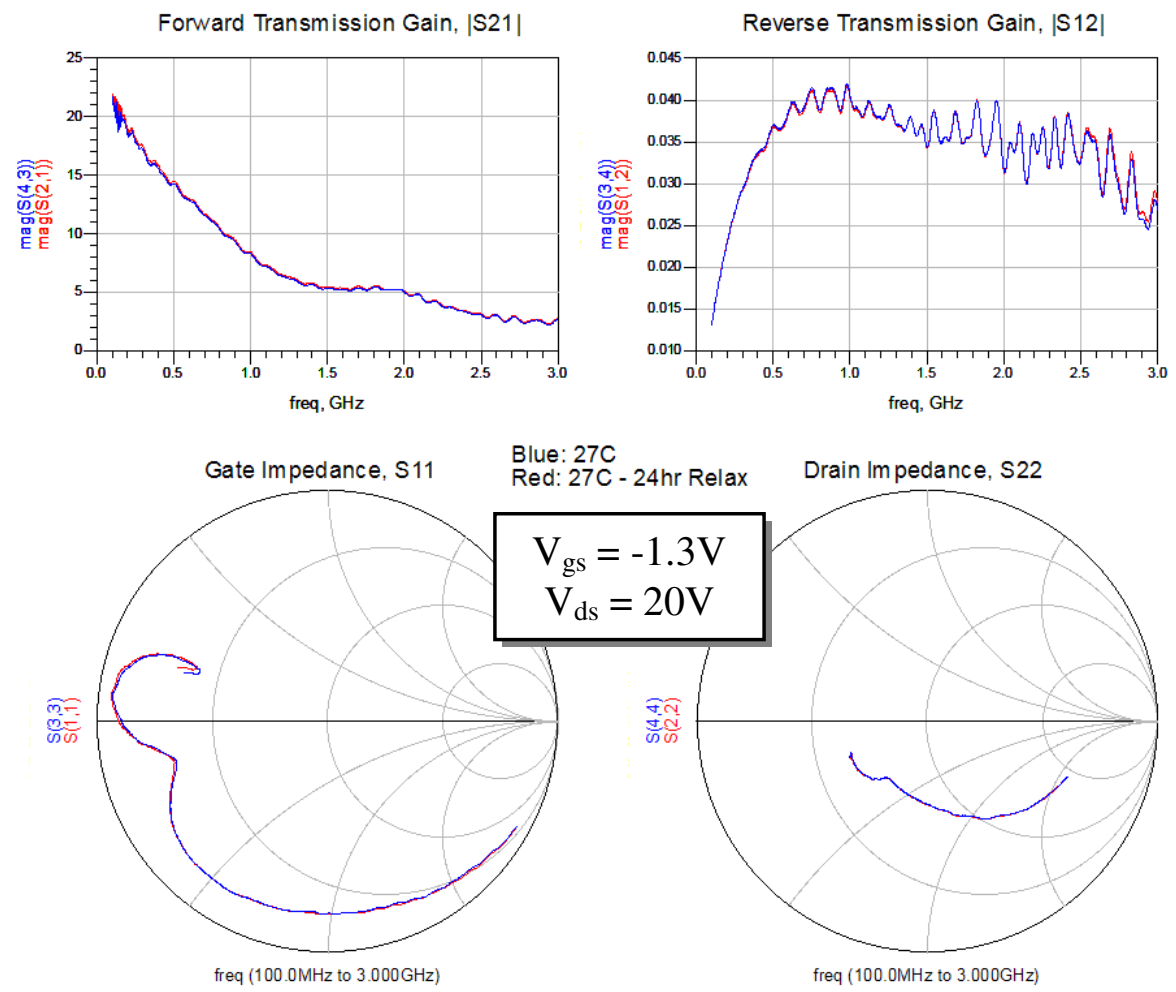

Figure 4.10 ? S-parameter comparison between a DUT at $27^{\circ} \mathrm{C}$ and at $27^{\circ} \mathrm{C} 24$ hours after thermal exposure at $100^{\circ} \mathrm{C}$.

\subsection{Effect of Temperature on the Critical Voltage}

\subsubsection{Setup}

All experiments performed in this section utilized the same test structures shown in Figure 4.1. The experiment was performed by first assigning a constant back-plate temperature to each device, and then proceeding with the step-stress procedure until the device's $\mathrm{V}_{\text {crit }}$ value was achieved. Devices were assigned the following temperatures and stressed accordingly: $27^{\circ} \mathrm{C}, 40^{\circ} \mathrm{C}, 50^{\circ} \mathrm{C}, 60^{\circ} \mathrm{C}, 70^{\circ} \mathrm{C}, 80^{\circ} \mathrm{C}, 90^{\circ} \mathrm{C}$, and $100^{\circ} \mathrm{C}$. 


\subsubsection{Results and Analyses}

The temperature dependent $\mathrm{V}_{\text {crit }}$ results for eight DUTs are displayed in Figure 4.11 while Figure 4.12 shows the change in junction temperature during the step-stress procedure for the eight devices. An observable reduction in $\mathrm{V}_{\text {crit }}$ was found as the backplate temperature was increased and a rise in junction temperature was found as each the gate-source voltage for each device approached $\mathrm{V}_{\text {crit }}$. Similar trends seen in Figure 4.11 were observed in [37] and [38], where $\mathrm{V}_{\text {crit }}$ had also become less negative with increasing temperature. An explanation for the negative temperature dependence of $\mathrm{V}_{\text {crit }}$ may be due to a breakdown in an oxide layer $\left(\beta-\mathrm{Ga}_{2} \mathrm{O}_{3}\right)$ that forms when thermal oxidation of $\mathrm{GaN}$ occurs [37].

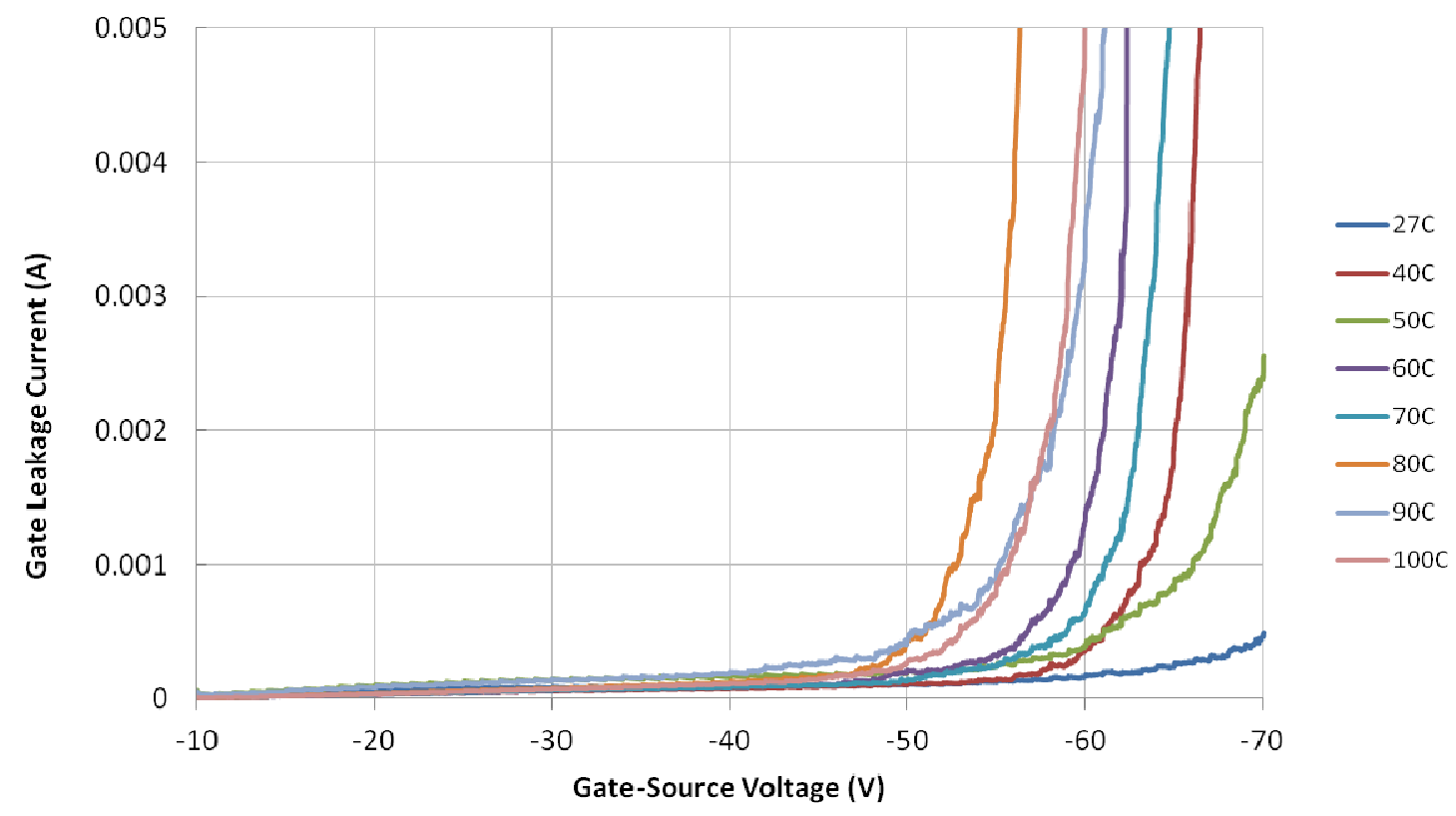

Figure 4.11 ? Shifts in the critical voltage $\left(\mathrm{V}_{\text {crit }}\right)$ parameter with varying back-plate temperatures. 


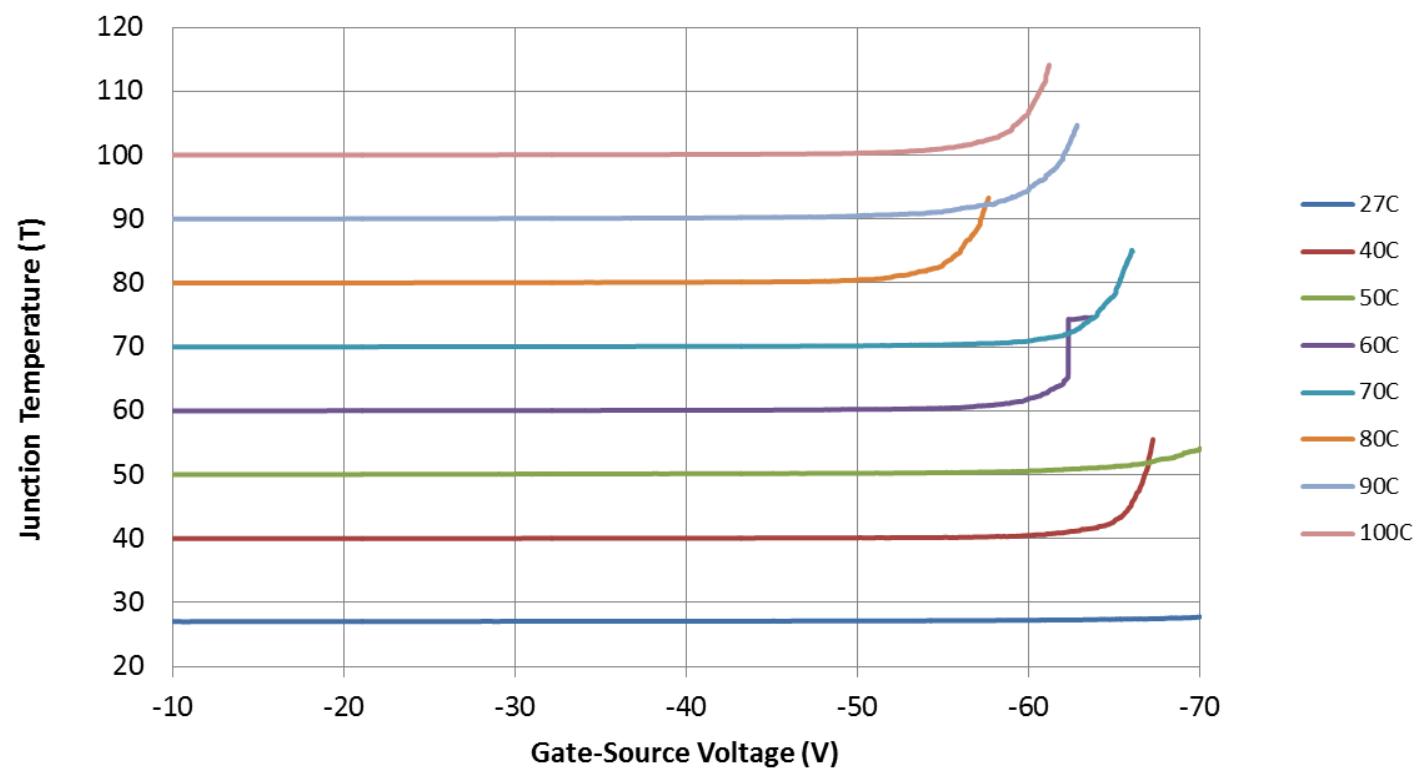

Figure 4.12 $\square$ Change in junction temperature as the gate-source voltage approaches Vcrit.

The ionic bond formed between Gallium and Oxygen can be severed under high thermal strain resulting in a breakdown of the oxide, which in turn promotes gate leakage. Work in [37] utilized a GaN cap layer between the Schottky gate contact of the device and the AlGaN layer. The devices used in this study also possess a GaN cap and may therefore be experiencing the same oxide creation and breakdown mechanisms causing the observed negative temperature dependence of $\mathrm{V}_{\text {crit. }}$.

An Arrhenius plot can be created from data similar to Figure 4.11 in order to determine the activation energies associated with the negative temperature-dependent critical voltage measurements. Unfortunately, due to the non-montomic nature of the data set of Figure 4.11 and a lack of finer temperature intervals, accurate Arrhenius plots could not be produced. 


\section{Experiment 3: Electron Trapping and Detrapping}

\subsection{Trapping Experiment A: On-State Operation}

\subsubsection{Setup}

Each DUT was assembled into a test configuration similar to that of the devices used in the temperature stress experiments (Figure 4.1, pg. 49). All devices in this experiment were shielded from light sources and the procedures were performed in dark rooms (no illumination) to prevent sunlight from acting as an electron detrapping source. The devices were first run under normal operation at $\mathrm{V}_{\mathrm{gs}}=-1.3 \mathrm{~V}$ and $\mathrm{V}_{\mathrm{ds}}=20 \mathrm{~V}$ to induce electron trapping and to assess the overall nature of the gate and drain current transients over a span of 1,000 seconds, a testing interval established in [39].

After monitoring the current transients for each transistor during normal operation, the devices were then biased at $\mathrm{V}_{\mathrm{gs}}=-1.3 \mathrm{~V}$ and $\mathrm{V}_{\mathrm{ds}}=-2 \mathrm{~V}$ to allow for conduction within the 2DEG channel to occur and to also restrict current flow to prevent self-heating in each DUT. The back-plate of each DUT was then increased from room temperature to $80^{\circ} \mathrm{C}$ to while the gate and drain current transients were monitored for electron detrapping events. The detrapping procedures were performed until the measured drain current transients stabilized.

\subsubsection{Results and Analyses}

A decreasing trend in both drain and gate current were measured during the onstate current transient experiments (Figure 5.1). For the device shown in Figure 5.1, the drain current reduced by $6.5 \%$ while the gate current decreased by $23.5 \%$ over a time period of 1,000 seconds. The collapse in drain and gate current seen in Figure 5.1 results 
from hot-electron injection into the AlGaN layer or the existence of surface traps [39]. Also suggested in [39] was the occurrence of deep-level traps within the GaN buffer, which further contributed to drain current collapse via depletion of the 2DEG.

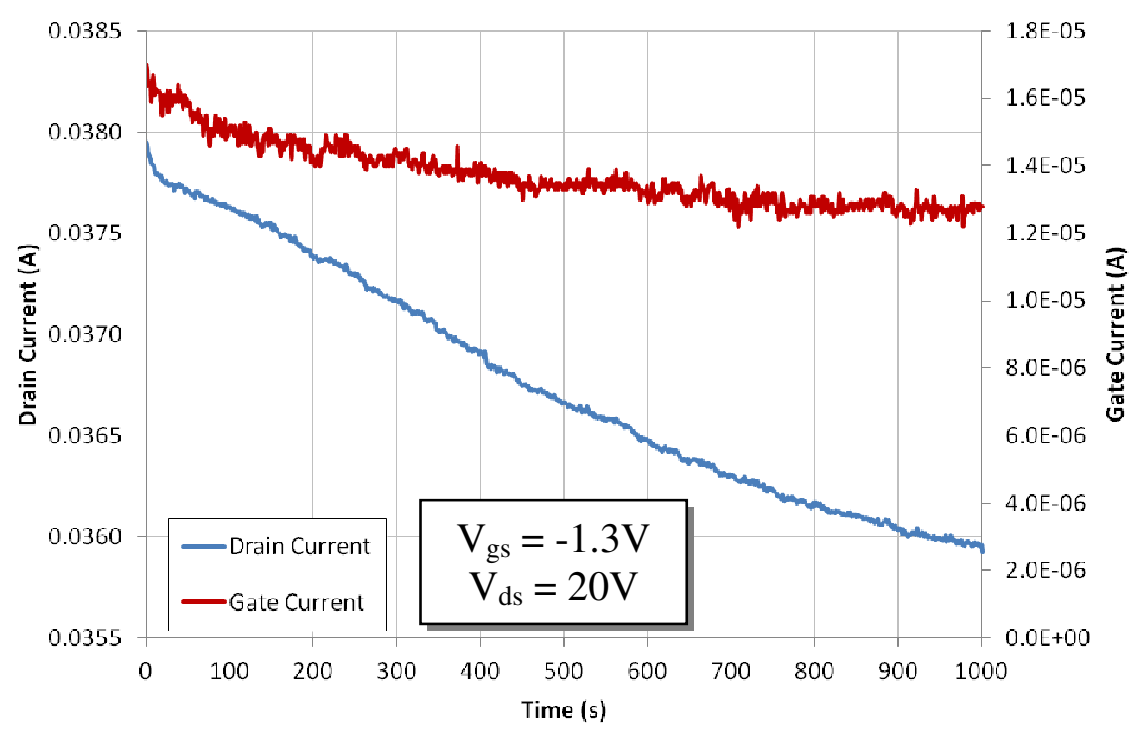

Figure 5.1 ? Drain and gate current transients of a DUT biased in the saturation region over a 1,000 second span.

Drain and gate current transients for two devices (Device A and Device B) exposed to rising back-plate temperatures are shown in Figure 5.2 and Figure 5.3, respectively. In the graphs of Figure 5.2, both devices displayed two observable peaks in drain current. For Device A, the peaks occur at back-plate temperatures of $30^{\circ} \mathrm{C}$ and $70^{\circ} \mathrm{C}$ while for Device B the peaks occur at $35^{\circ} \mathrm{C}$ and $75^{\circ} \mathrm{C}$. Another interesting observation was how the transition to the second detrapping peak for both devices occurred at backplate temperatures of approximately $50^{\circ} \mathrm{C}$ (indicated by the dashed green circles in Figure 5.2) The sudden recovery in drain current for both devices may be an indication of electron detrapping events that are thermally activated. In [39] and [40], it was found that 
the temperature-dependent detrapping events correlate with electrons trapped within the AlGaN layer of the HEMT devices.

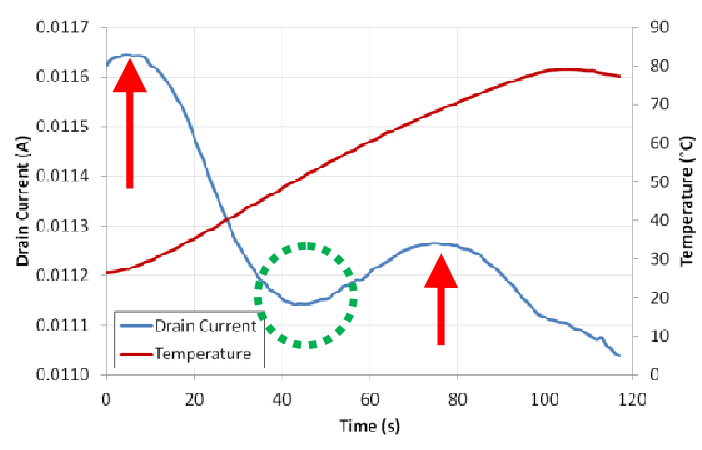

(a)

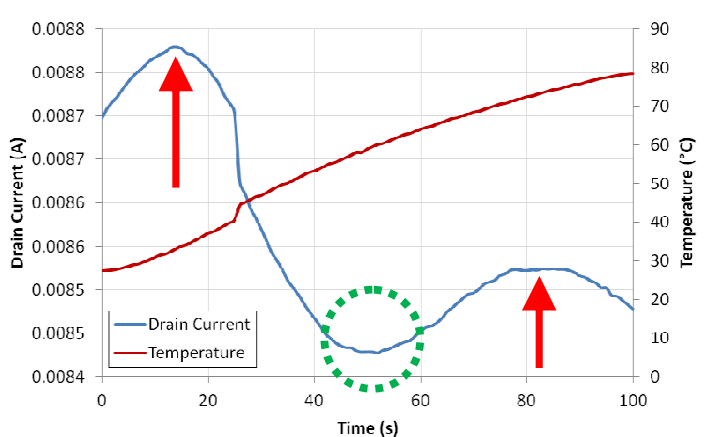

(b)

Figure 5.2 $\square$ Detrapping experiment drain current transient results for (a) Device A and (b) Device B $\left(\mathrm{V}_{\mathrm{gs}}=-1.3 \mathrm{~V}, \mathrm{~V}_{\mathrm{ds}}=2 \mathrm{~V}\right)$.

In Figure 5.3, both devices experienced a general rise in gate current with respect to increasing back-plate temperatures. The result could be due in part to the nature of the electron trapping and detrapping events localized within the $\mathrm{AlGaN}$ layer and close to the surface of the device. An electron trapping and detrapping analysis performed in [39] found that the activation energies associated with observed electron trapping and detrapping events was approximately equal to the Schottky barrier height of the experimental HEMT devices. Therefore, if electrons tunneling through the AlGaN buffer are presented with energy equivalent or greater than that of the Schottky barrier height, the barrier can be overcome. By overcoming the barrier height, trap-assisted tunneling (TAT) can take place and gate leakage current is enhanced as a function of increasing temperature [33]. 


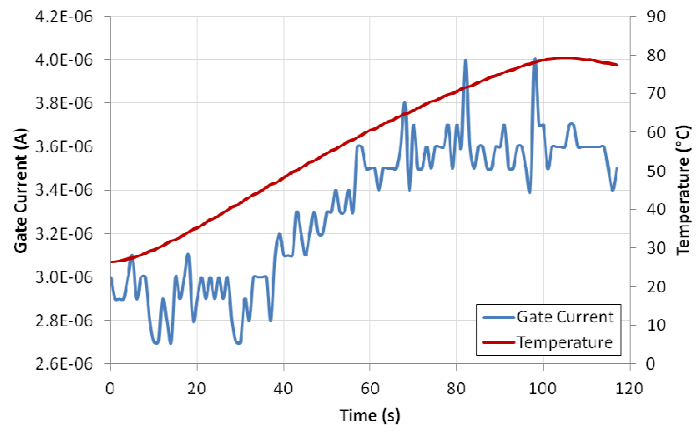

(a)

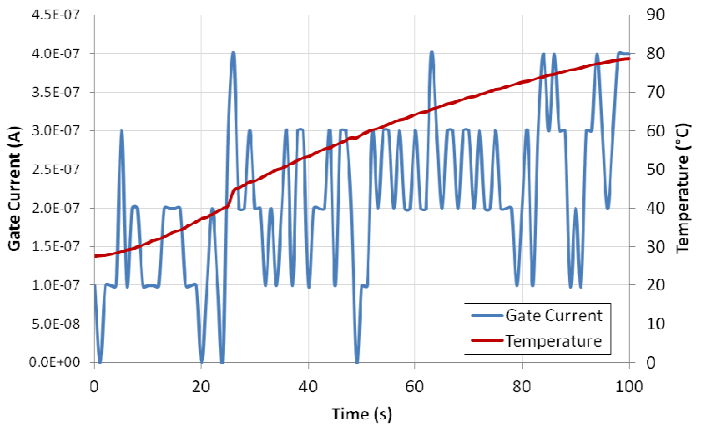

(b)

Figure 5.3 $\square$ Detrapping experiment gate current transient results for (a) Device A and (b) Device B $\left(\mathrm{V}_{\mathrm{gs}}=-1.3 \mathrm{~V}, \mathrm{~V}_{\mathrm{ds}}=2 \mathrm{~V}\right)$.

\subsection{Trapping Experiment B: High Electric Field Stress}

\subsubsection{Setup}

Similar procedures from Section 5.1 were used in this section to perform the electron detrapping analyses. Rather than inducing electron trapping events, however, the experiment was replaced with the symmetrical high-field stress procedure used in Section 3.2. The devices in this experiment were subjected to gate-source voltages of $\mathrm{V}_{\mathrm{gs}}=\mathrm{V}_{\text {crit }}=-70 \mathrm{~V}$ until the gate current leakage limit of $10 \mathrm{~mA}$ was achieved.

After subjecting each DUT to high-field stresses at $\mathrm{V}_{\mathrm{gs}}=\mathrm{V}_{\text {crit }}$, the devices were then biased at $\mathrm{V}_{\mathrm{gs}}=-1.3 \mathrm{~V}$ and $\mathrm{V}_{\mathrm{ds}}=-2 \mathrm{~V}$ to allow for conduction within the 2DEG channel to occur and to also restrict current flow to prevent self-heating in each DUT. The back-plate of each DUT was then increased from room temperature to $80^{\circ} \mathrm{C}$ to while the gate and drain current transients were monitored for electron detrapping events. The detrapping procedures were performed until the measured drain current transients stabilized. 


\subsubsection{Results and Analyses}

A representative measurement from the constant $V_{\text {crit }}$ bias experiment is shown in Figure 5.4. A linear trend was observed in the measured gate leakage current, similar to what was seen in Figure 3.7 (pg. 38). However, as explained in Subsection 3.2.2, the rate at which the gate leakage current increases varies between devices. The gate leakage current for the device in Figure 3.7 increased at a rate of $25.641 \mu \mathrm{A} / \mathrm{s}$ whereas the gate leakage current for the device in Figure 5.4 increased at a rate of $2.368 \mu \mathrm{A} / \mathrm{s}$. As expected from the results of the symmetric high-field stress experiments in Subsection 3.2.2, each DUT experienced an increase in saturation current similar to what was seen in Figure 3.8 (pg. 38).

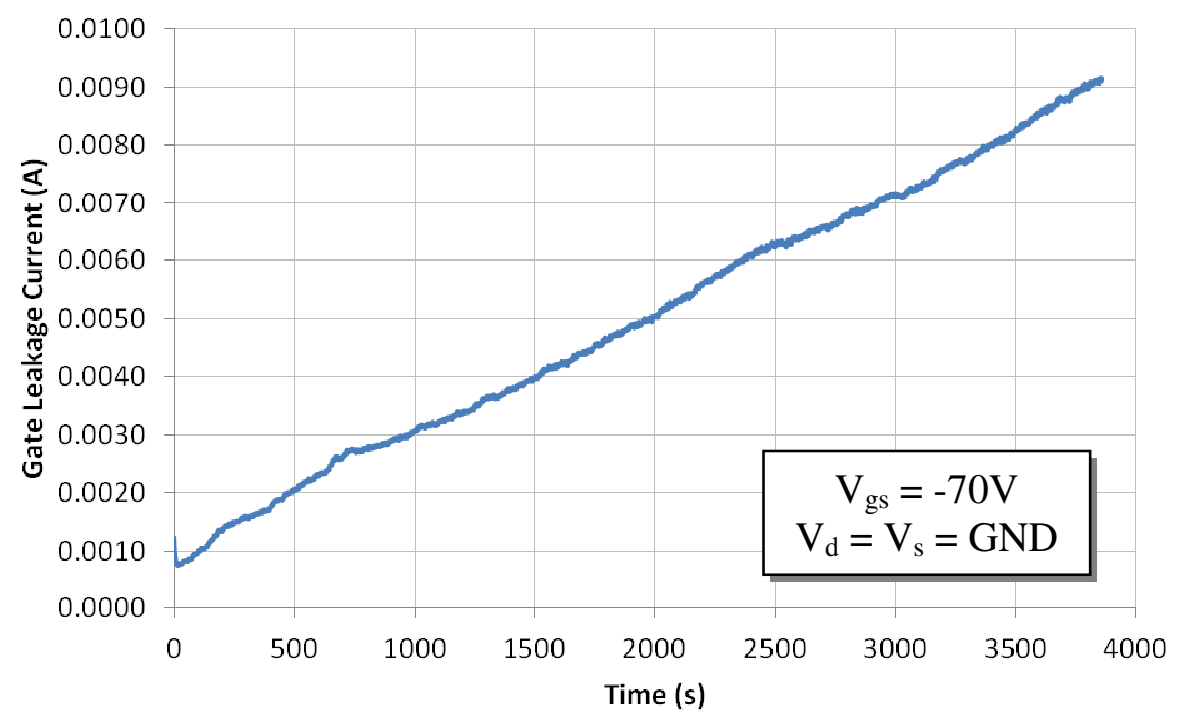

Figure 5.4 $\square$ Results of symmetric high-field stress at $\mathrm{V}_{\text {crit. }}$.

Figure 5.5 and Figure 5.6 show results similar to what was found in the symmetric high-field step-stress experiments in Section 3.2. An increase in both drain current and gate current were measured post-stress. To reiterate, the increased drain current was determined to be attributed to increased tensile strain at the heterojunction due to the 
application of the high symmetrical electric field distribution. The increase in gate current was said to be due to a lowering of the Schottky barrier height with respect to increasing vertical electric fields present at the metal-on-semiconductor interface.

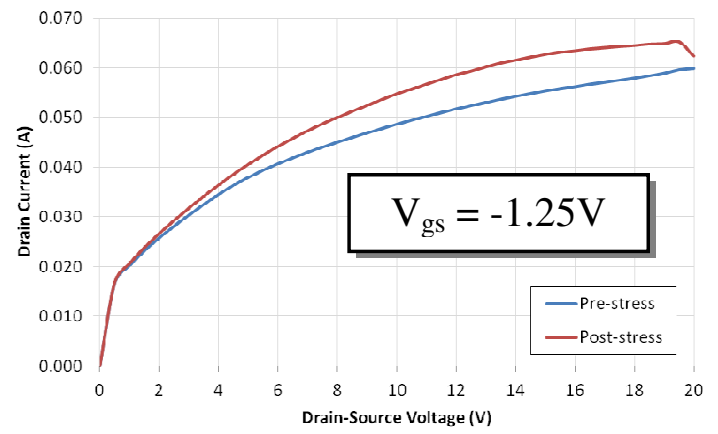

(a)

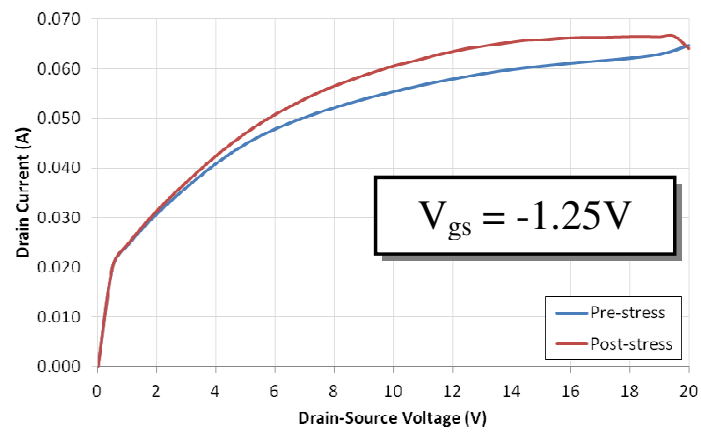

(b)

Figure 5.5 $\square$ Drain current characteristics before and after high-field stress for (a) Device C and (b) Device D.

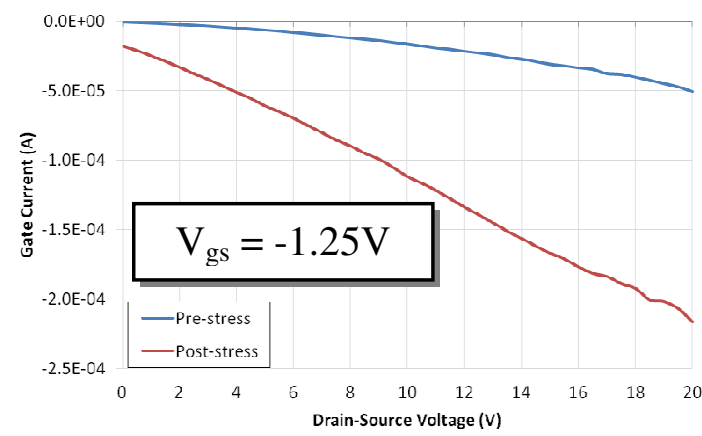

(a)

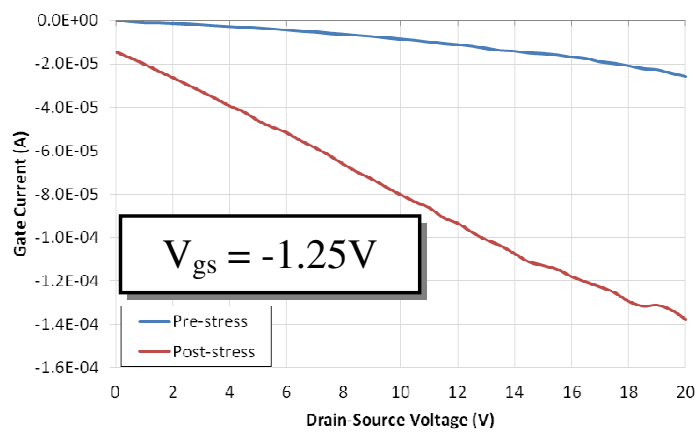

(b)

Figure 5.6 Gate current characteristics before and after high-field stress for (a) Device C and (b) Device D.

The thermal detrapping results for two devices are shown in Figure 5.7. Both devices showed a rapid decrease in drain current upon biasing each device in the linear region. The rapid reduction in drain current seen in the two transient plots was an indication of electron trapping, similar to the electron trapping results of Figure 5.1. The sharp decrease in current further supports the claim that there are process-dependent defects already existent within these devices prior to applying excitation sources. 


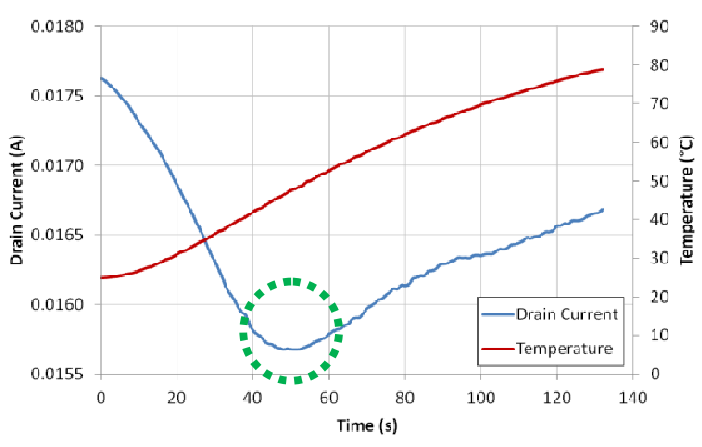

(a)

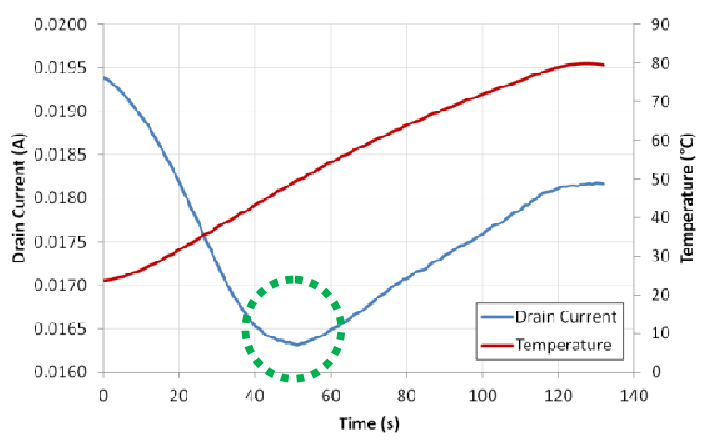

(b)

Figure 5.7 ? Detrapping experiment drain current transient results for (a) Device C and (b) Device D $\left(\mathrm{V}_{\mathrm{gs}}=-1.3 \mathrm{~V}, \mathrm{~V}_{\mathrm{ds}}=2 \mathrm{~V}\right)$.

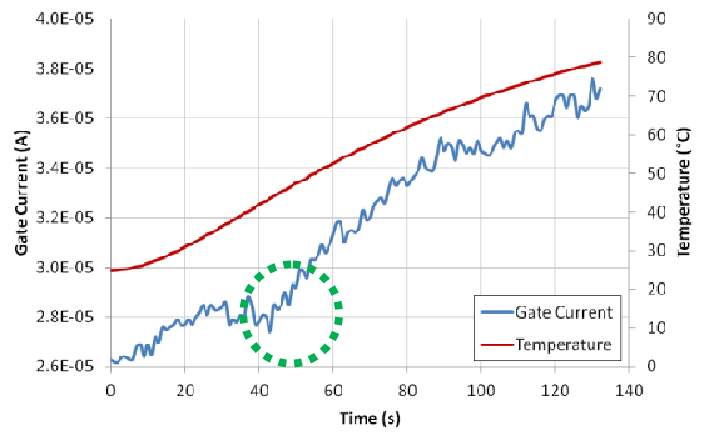

(a)

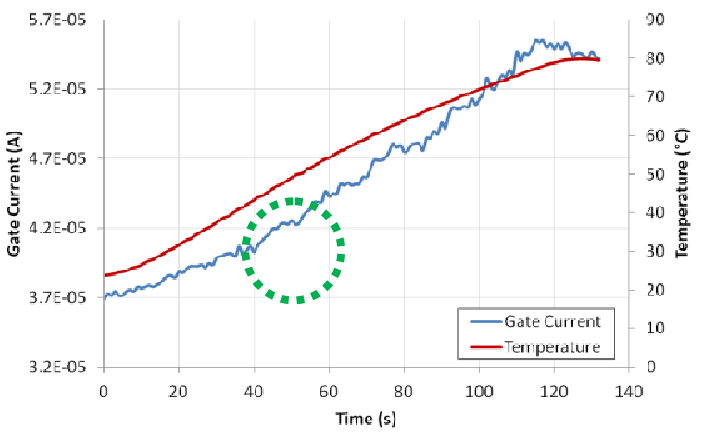

(b)

Figure 5.8 ? Detrapping experiment gate current transient results for (a) Device C and (b) Device D $\left(\mathrm{V}_{\mathrm{gs}}=-1.3 \mathrm{~V}, \mathrm{~V}_{\mathrm{ds}}=2 \mathrm{~V}\right)$.

An interesting observation, however, was how both devices began to experience a recovery in drain current when the back-plate temperatures of each DUT had reached $50^{\circ} \mathrm{C}$ (indicated by the dashed green circles in Figure 5.7). The same effect was seen during the experiments of Subsection 5.1.2, which may indicate that there is a specific activation energy associated with the electron detrapping event that takes place. The drain current characteristics for each HEMT continued to increase until the back-plate temperatures stabilized at $80^{\circ} \mathrm{C}$. Gate current transients were also measured under increasing back-plate temperatures and are shown in Figure 5.8. While the electron 
detrapping event is not as noticeable in Figure 5.8, the rate of increase in gate current does increase around a back-plate temperature of $50^{\circ} \mathrm{C}$. 


\section{Experiment 4: Small-Signal Analysis}

\subsection{Small-Signal Model Development in Agilent ADS}

\subsubsection{Setup}

The small-signal model for transistors usually consists of two parts: intrinsic parameters and extrinsic parameters [30]. The intrinsic parameters are the characteristics inherent to the die-form of the transistor, while the extrinsic parameters take into account the parasitics introduced after contact formation and packaging. A small-signal representation of a FET is shown in Figure 6.1 while the associated parameter definitions are shown in Table 6.1. Because a HEMT is classified as a metal semiconductor fieldeffect transistor (MESFET) due to having metalized contacts and a Schottky barrier gate contact, the device can also utilize the same small-signal representation. A fit of the intrinsic parameters to the cross-sectional geometry of the GaN-on-Si HEMT is shown in Figure 6.2.

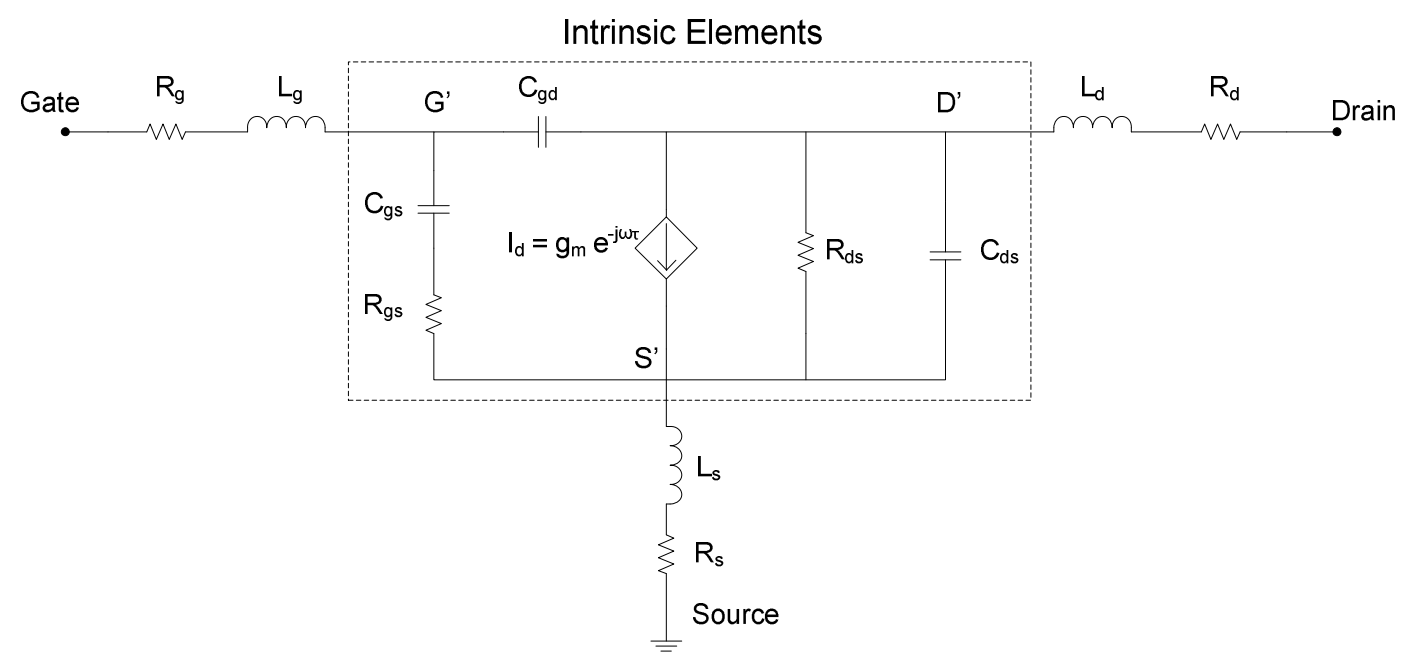

Figure 6.1 $\square$ Small-signal model circuit with both intrinsic and extrinsic parameters. 
Table 6.1 ? Small-signal parameter definitions.

\begin{tabular}{|c|c|}
\hline \multicolumn{2}{|c|}{ Small-Signal Parameters } \\
\hline Extrinsic & Intrinsic \\
\hline Gate Resistance $\left(\mathrm{R}_{\mathrm{g}}\right)$ & Gate-Source Resistance $\left(\mathrm{R}_{\mathrm{gs}}\right)$ \\
\hline Source Resistance $\left(\mathrm{R}_{\mathrm{s}}\right)$ & Drain-Source Resistance $\left(\mathrm{R}_{\mathrm{ds}}\right)$ \\
\hline Drain Resistance $\left(\mathrm{R}_{\mathrm{d}}\right)$ & Gate-Source Capacitance $\left(\mathrm{C}_{\mathrm{gs}}\right)$ \\
\hline Gate Inductance $\left(\mathrm{L}_{\mathrm{g}}\right)$ & Gate-Drain Capacitance $\left(\mathrm{C}_{\mathrm{gd}}\right)$ \\
\hline Source Inductance $\left(\mathrm{L}_{\mathrm{s}}\right)$ & Drain-Source Capacitance $\left(\mathrm{C}_{\mathrm{ds}}\right)$ \\
\hline Drain Inductance $\left(\mathrm{L}_{\mathrm{d}}\right)$ & Transconductance $\left(\mathrm{g}_{\mathrm{m}}\right)$ \\
\hline & Electron Transit Time $(\tau)$ \\
\hline
\end{tabular}

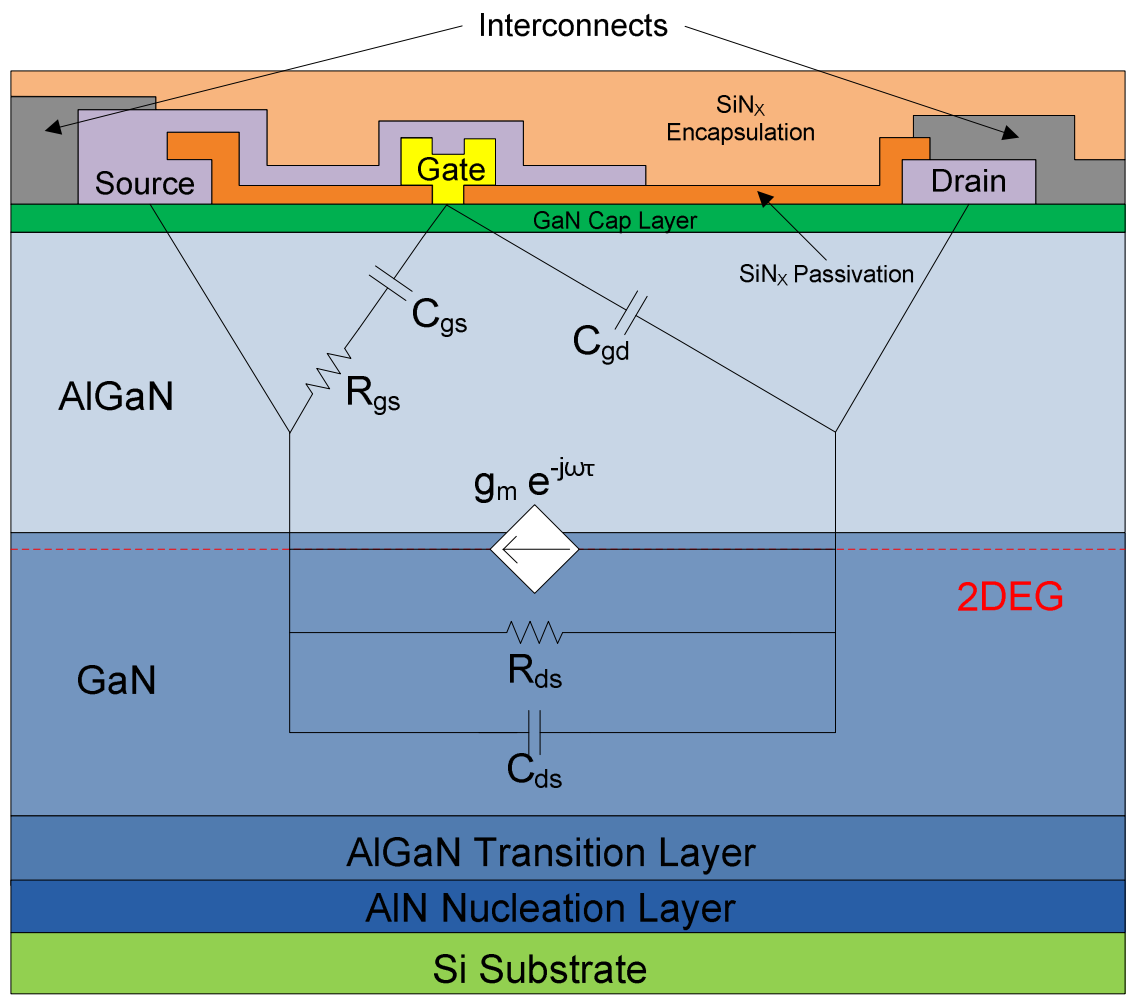

Figure 6.2 $\square$ Cross-sectional geometry of the GaN-on-Si DUT with intrinsic parameters overlaid.

$C_{g s}, R_{g s}, C_{d s}$ and $R_{d s}$ were estimated using the measured $S_{11}$ and $S_{22}$ data for the

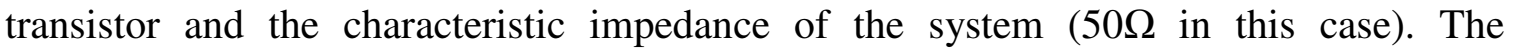
parameters were extracted using data at $100 \mathrm{MHz}$ to minimize contributions from 
parasitic inductances. The S-parameters were measured using an Anritsu MS4622B (using the test conditions in Subsection 2.2.3) and the input and output impedances were extracted in Agilent ADS by importing an S2P file from the VNA into the program.

$\mathrm{R}_{\mathrm{gs}}$ and $\mathrm{C}_{\mathrm{gs}}$ were first calculated using Equations (6.1) and (6.2). $\mathrm{C}_{\mathrm{dso}}$ in Equation (6.3) is the series capacitance associated with the imaginary part of the $S_{22}$ impedance. $\mathrm{C}_{\mathrm{dso}}$ will be used in a series to parallel transformation process to solve for the drain-source capacitance. $R_{d s}$ and $C_{d s}$ were calculated using the transformations of Equation (6.4) and Equation (6.5). More information on series to parallel and parallel to series transformations can be found in [41].

$$
\begin{gathered}
R_{g s}=Z_{o} \operatorname{Re}\left(z_{11}\right) \\
C_{g s}=\frac{1}{\omega Z_{o}\left|\operatorname{Im}\left(z_{11}\right)\right|} \\
C_{d s o}=\frac{1}{\omega Z_{o}\left|\operatorname{Im}\left(z_{22}\right)\right|} \\
R_{d s}=Z_{o} \operatorname{Re}\left(z_{22}\right)+\frac{1}{Z_{o} \operatorname{Re}\left(z_{22}\right) *\left(\omega C_{d s o}\right)^{2}} \\
C_{d s}=\frac{1}{Z_{o} \operatorname{Re}\left(z_{22}\right) * R_{d s} \omega^{2} C_{d s o}}
\end{gathered}
$$

The value of $g_{m}$ was estimated using a transistor parameter analyzer and the electron transient time associated with the drain current, $\tau$, is a parameter that was tuned 
using Agilent ADS. The remaining parasitic components were tuned in Agilent ADS until the small-signal model S-parameter data coincided with the measured data.

\subsubsection{Results and Analyses}

A generic small-signal model (Figure 6.3) was first created and replicated three times in order to produce pre-stress, post-stress, and recovery conditions (refer to Chapter 3.2.3). Before performing the S-parameter data fits, an S2P file with the data for the previously mentioned stress conditions were imported into Agilent ADS in order to act as a template by which the simulated S-parameters could be fit to.

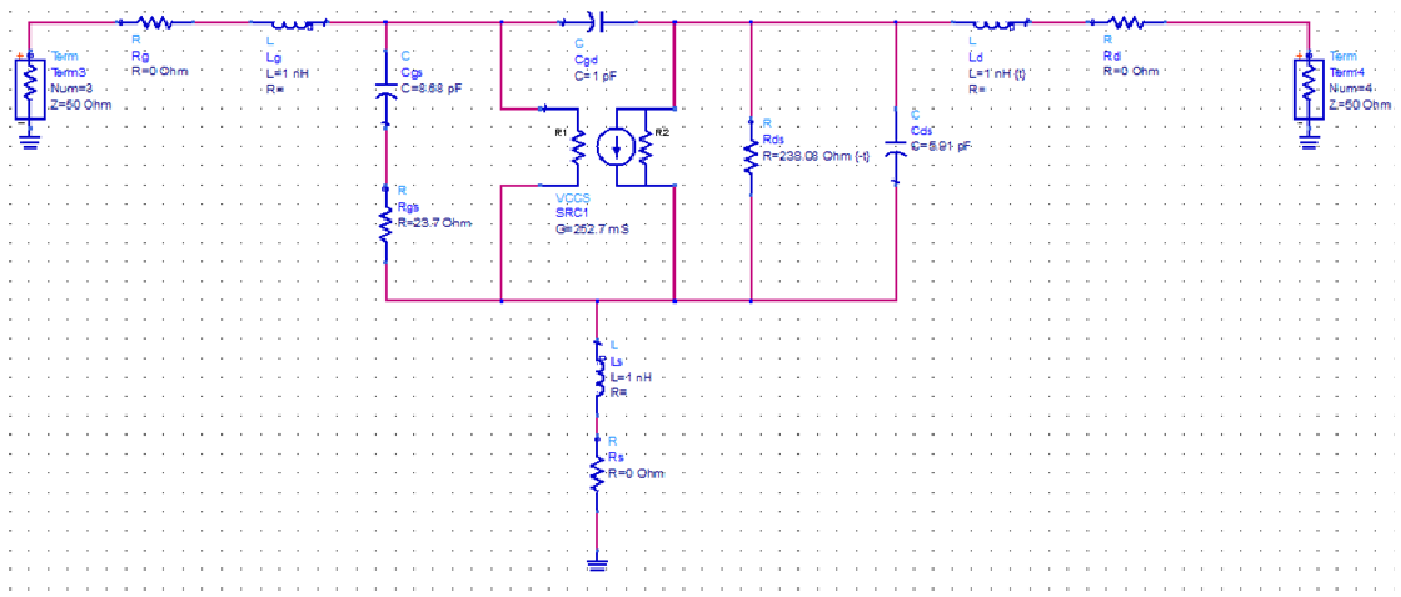

Figure 6.3 [ Constructed small-signal model using Agilent ADS.

The intrinsic section for each of the three small-signal models used in the fitting process were developed using initial $\mathrm{DC}$ and $\mathrm{RF}$ measurements in conjunction with Equations (6.1) through (6.5) (refer to the previous subsection). After placing the estimated values for each intrinsic component, the extrinsic values were then set to arbitrary values. Without tuning, S-parameters plots similar to Figure 6.4 would result. Next, the "Tune Parameters" interface was used to manually adjust simulated smallsignal parameters until the associated model began to correlate with the measured data. 
The tuning interface is shown in Figure 6.5 while an example of an approximated data fit is shown in Figure 6.6.
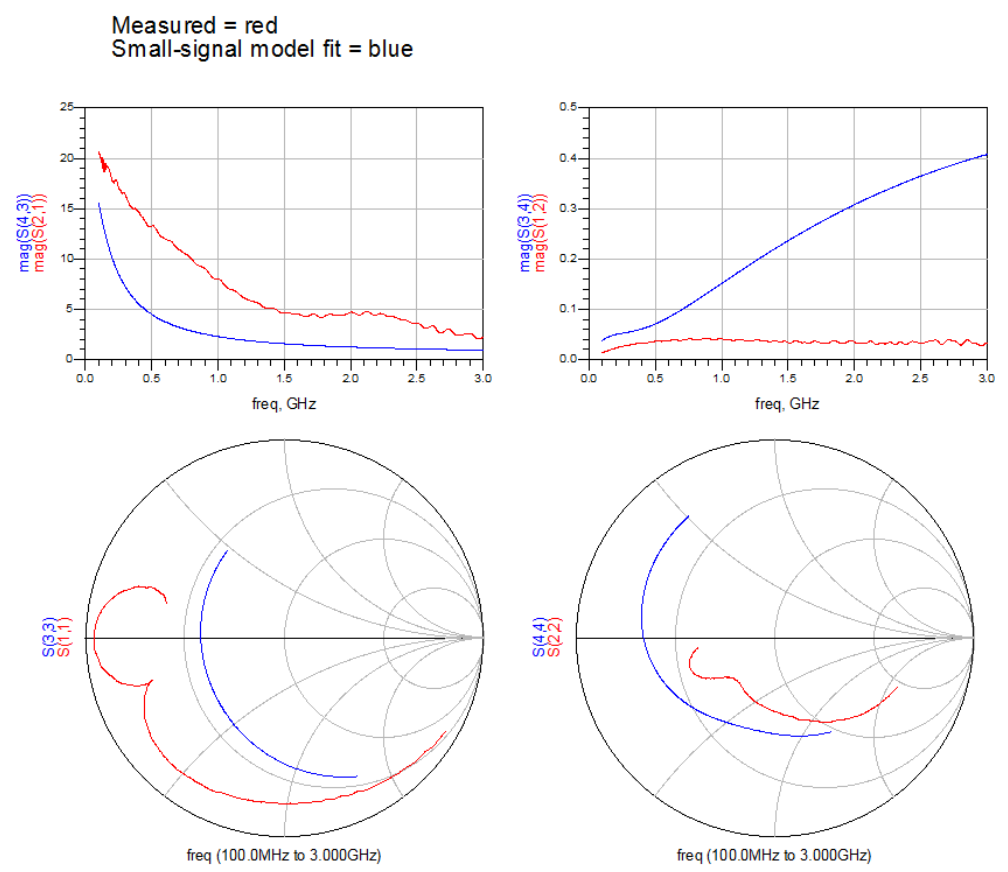

Figure 6.4 ? Simulated S-parameters prior to fitting routine.

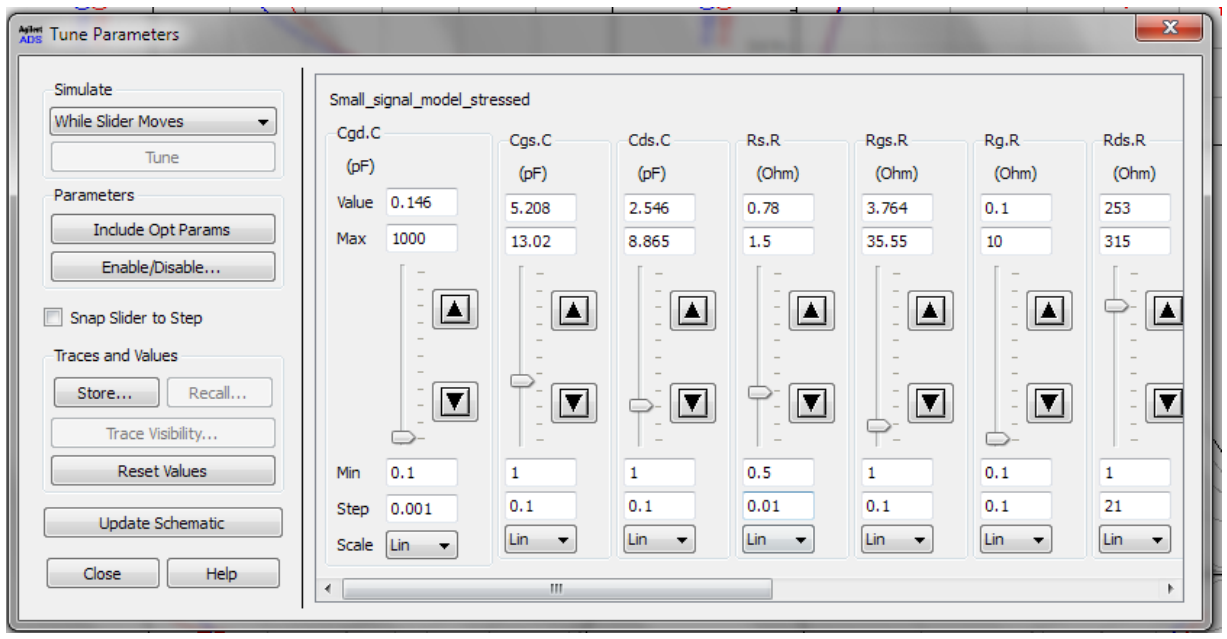

Figure 6.5 ? Agilent ADS “Tune Parameter” interface. 

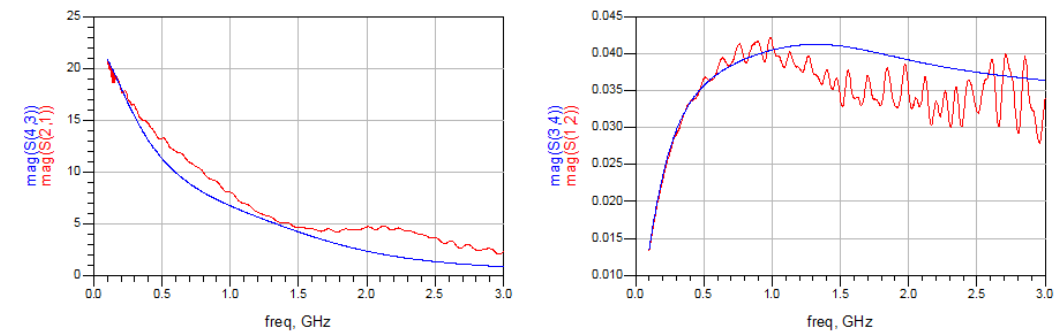

Measured $=$ red

Small-signal model fit $=$ blue
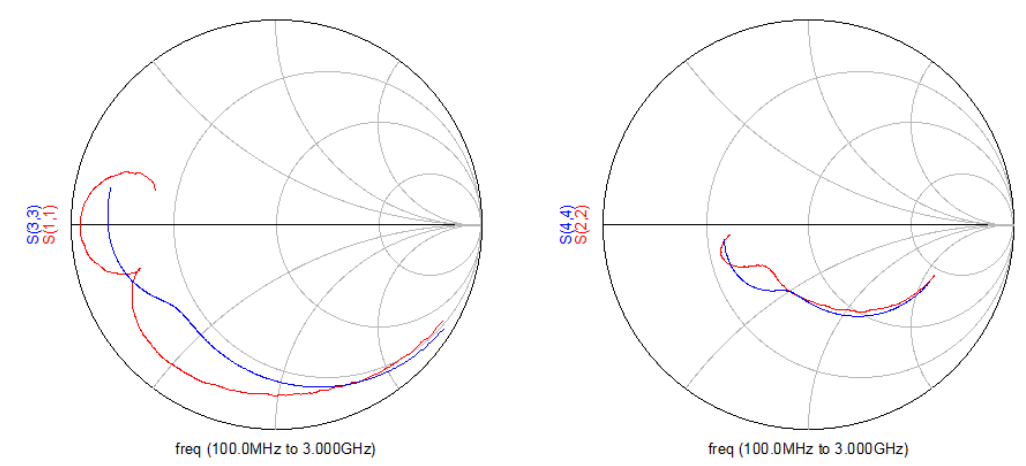

Figure 6.6 [ Simulated S-parameters after using the parameter tuning interface in ADS.

Small-signal model fits for each of the three DUT conditions were developed using the previously mentioned tuning schemes. Imported S-parameter data for the three DUT conditions are shown in Figure 6.7 while the simulated S-parameters resulting from the small-signal parameter fit to the measured data are shown in Figure 6.8. While not entirely accurate, the resulting data approximations of Figure 6.8 allow for estimations as to how the small-signal parameters for a DUT change between pre-stress, post-stress, and relaxed states. Major changes in small-signal parameters between each of the three DUT conditions are displayed in Table 6.2. 


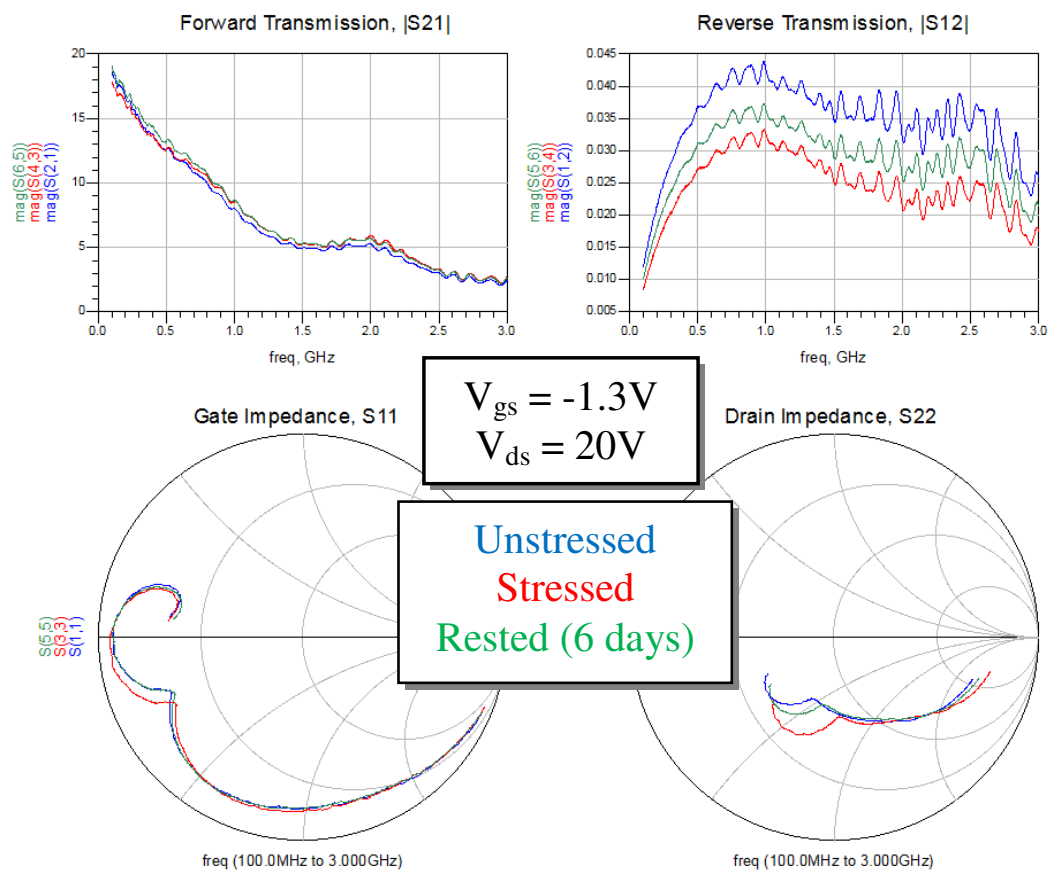

Figure 6.7 $\square$ Measured S-parameters used for small-signal modeling.
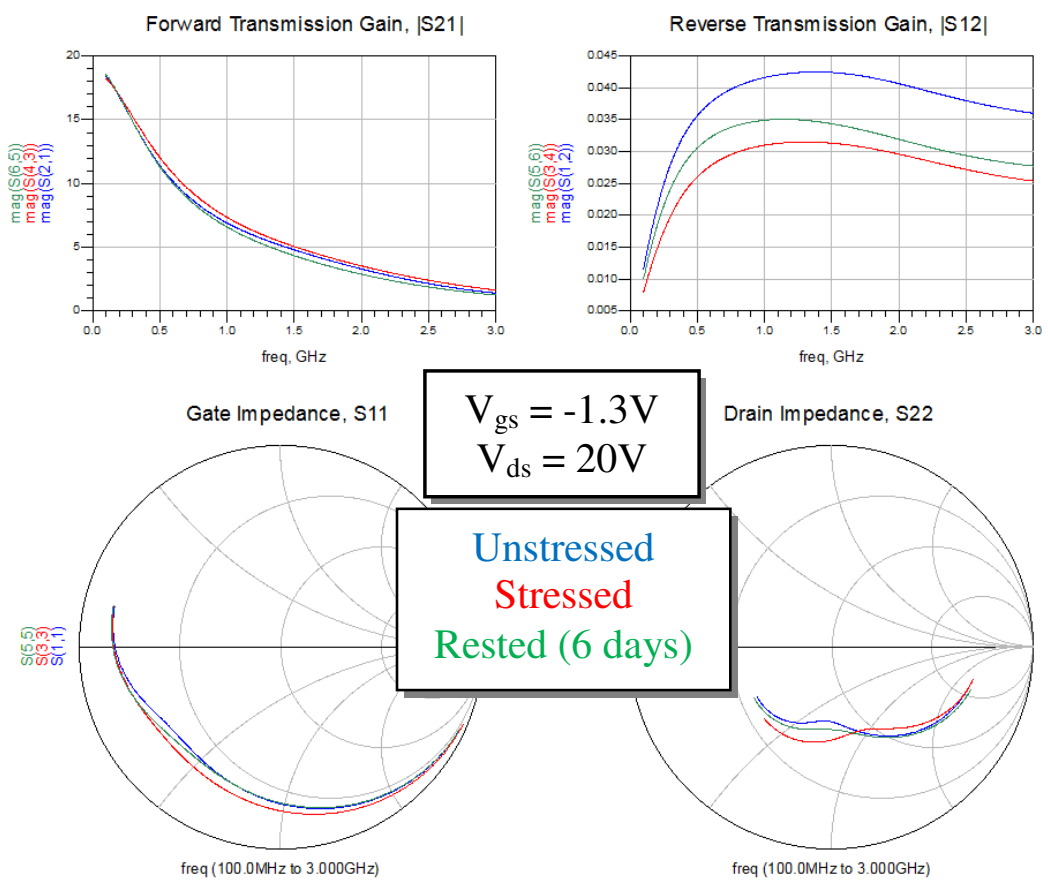

Figure 6.8 ? Simulated S-parameters after performing a small-signal parameter fit to measured data. 
Table 6.2 ? Major small-signal parameter changes for pre-stress, post-stress, and rested HEMT test conditions.

\begin{tabular}{|r|c|c|c|c|}
\hline & \multicolumn{4}{|c|}{ Major Change in Small-Signal Parameters } \\
\hline Stress State & $\begin{array}{c}\text { Source } \\
\text { Resistance }(\boldsymbol{\Omega})\end{array}$ & $\begin{array}{c}\text { Gate-Drain } \\
\text { Capacitance }(\boldsymbol{p} F)\end{array}$ & $\begin{array}{c}\text { Drain-Source } \\
\text { Capacitance }(\boldsymbol{p} F)\end{array}$ & $\begin{array}{c}\text { Drain-Source } \\
\text { Resistance }(\boldsymbol{\Omega})\end{array}$ \\
\hline Unstressed & 1.200 & 0.216 & 3.546 & 211 \\
\hline Stressed & $0.78(35.00 \%)$ & $0.146(32.41 \%)$ & $2.546(28.20 \%)$ & $253(19.91 \%)$ \\
\hline Rested (6 days) & $0.890(25.83 \%)$ & $0.188(12.96 \%)$ & $3.546(0.00 \%)$ & $253(19.91 \%)$ \\
\hline
\end{tabular}

As seen above, the source resistance $\left(R_{s}\right)$, the gate-drain capacitance $\left(C_{g d}\right)$, the drain-source capacitance $\left(C_{d s}\right)$, and the drain-source resistance $\left(R_{d s}\right)$ experienced the most substantial changes amongst the simulated small-signal parameters. As was expected from Subsection 3.2.2, $\mathrm{C}_{\mathrm{gd}}$ reduced by $32.41 \%$ after the HEMT had been exposed to high symmetric-field stress. In general, a capacitor can be defined by Equation (6.6) [42]:

$$
C=\frac{Q}{V}
$$

Given that the voltage distribution across the capacitor is constant, as the charge (Q) stored in the capacitor changes, the capacitance changes proportionally. Because $\mathrm{C}_{\mathrm{gd}}$ is experiencing a reduction in capacitance after being exposed to a fixed high field stress, the only direct cause would be due to a reduction in charge stored in the AlGaN barrier. Therefore, the results may indicate how inducing the inverse piezoelectric effect in the GaN-on-Si HEMTs promotes the detrapping of electrons inherent from processdependent defects created during the growth of the epitaxial layers. Furthermore, a $12.96 \%$ recovery in $\mathrm{C}_{\mathrm{gd}}$ not only indicates how native defects in the device promote electron trapping, but how degradation in the AlGaN barrier induced by high field stress is not entirely permanent.

DC stress experiments performed in [25] on GaN-on-Si HEMTs have demonstrated non-permanent changes in the drain resistance $\left(\mathrm{R}_{\mathrm{d}}\right)$ and the source 
resistance $\left(R_{s}\right)$ when the devices under test were exposed to voltages below $V_{\text {crit. }}$ When $\mathrm{V}_{\mathrm{gs}}$ was set equal to or greater than $\mathrm{V}_{\text {crit }}$, the changes to the parameters were irreversible. While $R_{d}$ did not appear to experience any significant alterations over the test frequency range after high field stress, $\mathrm{R}_{\mathrm{s}}$ increased by approximately $35 \%$. While the DUTs were exposed to extensive periods of high voltage stressing at $V_{\text {crit }}$, the parameter $R_{s}$ was able to recover by $25.83 \%$, which further indicates that the inverse piezoelectric effect may not be the sole driving factor for permanent device degradation and defect formation in GaN-on-Si HEMTs.

Both the drain-source resistance $\left(\mathrm{R}_{\mathrm{ds}}\right)$ and capacitance $\left(\mathrm{C}_{\mathrm{ds}}\right)$ increase post-stress by $19.91 \%$ and $28.20 \%$, respectively. After a relaxation period of 6 days, $\mathrm{C}_{\mathrm{ds}}$ is able to make a full recovery. The value of $R_{d s}$, however, remained unchanged after the 6-day rest period after the DUT was exposed to high-field stress. From Equation (1.3) (pg. 5), an increase in $R_{d s}$ may have potentially contributed to a proportional increase in the $f_{\max }$ of the transistor. The previously mentioned results may be an indication that there are some defect formations in the $\mathrm{GaN}$ barrier following high field stress at the device's $\mathrm{V}_{\text {crit }}$ occurring concurrently with electron trapping and detrapping events. 


\section{Conclusion}

Over the course of the research, several mechanisms leading to either device degradation or recovery have been recorded and linked to high electric field-related conditions in GaN-on-Si HEMTs. The high-field stress experiments have shown that GaN-on-Si devices with epitaxial structures similar to Figure 2.1 should experience critical voltages around $-70 \mathrm{~V}$ while in a $\mathrm{V}_{\mathrm{ds}}=\mathrm{V}_{\mathrm{s}}=$ ground (symmetric) configuration and undergo rapid increases in gate leakage current at the onset of $\mathrm{V}_{\text {crit. }}$ Though the electrical characteristics of the HEMTs change with respect to biasing the devices at $\mathrm{V}_{\text {crit, }}$, the changes are not permanent and device characteristics are slightly recoverable. While observable changes occur in the symmetric off-state condition, no noticeable alterations were noticeable in the $\mathrm{V}_{\mathrm{gs}}=-5 \mathrm{~V}$ off-state (asymmetric) scenario.

Variations in ambient temperature were found to have a direct influence on the overall performance of the GaN-on-Si HEMTs. Increasing the back-plate temperatures of the devices alters their corresponding junction temperatures, which in turn degrades their DC and RF characteristics under increasing temperature. The observations are undesirable in a practical sense because slight temperature gradients in an ambient setting can shift the operating point of the transistors. For example, if the HEMTs are utilized in a power amplifier (PA) setting and multiple PAs are required for a specific application, the self-heating of the devices can directly influence an adjacent device's performance. Another issue stemming from temperature variation was the direct correlation between increasing base-plate temperatures and a reduction in the absolute value of $\mathrm{V}_{\text {crit. }}$. The observed positive shift in $\mathrm{V}_{\text {crit }}$ as a function of increasing base-plate temperature indicated 
that less gate drive is required to alter the characteristics of the devices, promote an increase in gate leakage current, and potentially force them into abrupt failure modes.

The analyses performed in Chapter 5 have further provided evidence of hot electron injection into the buffer layers while the devices are operating in the on-state. The detrapping experiments following the on-state analyses show specific back-plate temperatures at which drain current suddenly begins to recover. Similar detrapping observations were found when performing the same experiment on devices previously exposed to high electric fields. The aforementioned phenomena may indicate the existence of a specific activation energy at which electrons are released from buffer traps, which in turn leads to $2 \mathrm{DEG}$ recovery. Another important observation drawn from the studies of Chapter 5 (in conjunction with Chapter 3) was how high-field stress promotes (rather than inhibits) drain current recovery through symmetric tensile strain of the semiconductor materials and also assists in electron detrapping.

The small-signal analyses performed on stressed HEMTs revealed major changes to four specific small-signal parameters: source resistance $\left(\mathrm{R}_{\mathrm{s}}\right)$, gate-drain capacitance $\left(C_{g d}\right)$, drain-source capacitance $\left(C_{d s}\right)$, and drain-source resistance $\left(R_{d s}\right)$. The $R_{s}$ and $C_{g d}$ parameters of the transistors experienced reductions up to $30 \%$ due to stress and then underwent some partial recovery over a 6-day relaxation period. A reduction in $\mathrm{C}_{\mathrm{gd}}$ further supports the claim that the symmetric high field stresses applied to the devices promotes electron detrapping in the AlGaN through the inverse piezoelectric effect due to a reduction in localized charge. Enhanced unilateral performance also results from the high-field stress due to a reduction in $\mathrm{C}_{\mathrm{gd}}$, which in turn relates to the devices' reverse transmission gain. A reduction in $\mathrm{C}_{\mathrm{ds}}$ was observed post-stress with full recovery after 
relaxation. The only parameter to have experienced permanent alteration was $R_{d s}$, which may suggest some degradation within the GaN barrier of the HEMTs. Though the change

to $\mathrm{R}_{\mathrm{ds}}$ was permanent, the permanent increase to the resistance proportionally increases the maximum frequency of oscillation for the transistor as well.

Table 7.1 ? Summary of experimental results.

\begin{tabular}{|c|c|c|c|c|}
\hline \multirow[t]{2}{*}{ Exp. \# } & \multirow{2}{*}{$\begin{array}{l}\text { Chapter \# } \\
\text { and Page \# }\end{array}$} & \multicolumn{3}{|c|}{ Experimental Details } \\
\hline & & Exp. Name & Sub-Exp. Name & Results Summary \\
\hline \multirow[b]{2}{*}{1} & \multirow[b]{2}{*}{3 (pg. 32) } & \multirow[b]{2}{*}{$\begin{array}{l}\text { High Electric } \\
\text { Field Stress } \\
\text { Analyses }\end{array}$} & $\begin{array}{l}\text { Asymmetric Electric } \\
\text { Field Stress }\end{array}$ & $\begin{array}{l}\text { No significant changes to device characteristics } \\
\text { were observed after stressing the devices to } 120 \mathrm{~V} \\
\text { on the drain in the off-state. }\end{array}$ \\
\hline & & & $\begin{array}{l}\text { Symmetric Electric } \\
\text { Field Stress }\end{array}$ & $\begin{array}{l}\text { Critical voltage for the DUT determined to be } \mathrm{V}_{\mathrm{gs}} \\
=-70 \mathrm{~V} \text { in the } \mathrm{V}_{\mathrm{ds}}=0 \mathrm{~V} \text { off-state. Rapid increase } \\
\text { in gate current as } \mathrm{V}_{\mathrm{gs}} \text { approaches the critical } \\
\text { voltage. Changes to device characteristics post- } \\
\text { stress included the following: an increase in } \\
\text { saturation drain current, an increase in gate } \\
\text { current, negative threshold voltage shift, and a } \\
\text { reduction in } \mid \mathrm{S} 21 \mathrm{I} \text { (correlating to } \mathrm{C}_{\mathrm{gd}} \text { ). Noticeable } \\
\text { device recovery after an extended period of no } \\
\text { electrical excitation; characteristic changes post- } \\
\text { stress are not permanent. }\end{array}$ \\
\hline \multirow{2}{*}{2} & \multirow{2}{*}{4 (pg. 49) } & \multirow{2}{*}{$\begin{array}{c}\text { Temperature } \\
\text { Characterization }\end{array}$} & $\begin{array}{l}\text { Effect of } \\
\text { Temperature on } \\
\text { General Device } \\
\text { Performance }\end{array}$ & $\begin{array}{l}\text { A reduction in drain current, an increase in gate } \\
\text { current, a positive shift in threshold voltage, and } \\
\text { a rise in gate-drain capacitance observed. } \\
\text { Characteristic changes are non-permanent. }\end{array}$ \\
\hline & & & $\begin{array}{l}\text { Effect of } \\
\text { Temperature on the } \\
\text { Critical Voltage }\end{array}$ & $\begin{array}{l}\text { Critical voltage shows a negative temperature } \\
\text { dependence; absolute value of the critical voltage } \\
\text { decreases with increasing temperature. }\end{array}$ \\
\hline \multirow[t]{2}{*}{3} & \multirow[t]{2}{*}{5 (pg. 62) } & \multirow{2}{*}{$\begin{array}{l}\text { Electron } \\
\text { Trapping and } \\
\text { Detrapping }\end{array}$} & $\begin{array}{l}\text { Trapping Experiment } \\
\text { A: On-State } \\
\text { Operation }\end{array}$ & $\begin{array}{l}\text { On-state operation promotes electron trapping, } \\
\text { which is observable through the reduction in gate } \\
\text { and drain current transients. Observable recovery } \\
\text { events during the application of thermal } \\
\text { excitations points towards activations energies } \\
\text { associated with electron detrapping. }\end{array}$ \\
\hline & & & $\begin{array}{l}\text { Trapping Experiment } \\
\text { B: High Electric } \\
\text { Field Stress }\end{array}$ & $\begin{array}{l}\text { The test indicates that stressing the devices at the } \\
\text { critical voltage enhances device performance, } \\
\text { indicating possible electron detrapping during } \\
\text { stress. Further evidence of a specific activation } \\
\text { energy associated with electron detrapping. }\end{array}$ \\
\hline 4 & 6 (pg. 70) & $\begin{array}{l}\text { Small-Signal } \\
\text { Analysis }\end{array}$ & $\sim$ & $\begin{array}{l}\text { A reduction in source resistance }\left(\mathrm{R}_{\mathrm{s}}\right) \text {, a reduction } \\
\text { in gate-drain capacitance }\left(\mathrm{C}_{\mathrm{gd}}\right) \text {, a reduction in } \\
\text { drain-source capacitance }\left(\mathrm{C}_{\mathrm{ds}}\right) \text {, and an increase in } \\
\text { drain-source resistance were observed post-stress. } \\
\text { Only permanent parameter change was observed } \\
\text { with drain-source resistance. }\end{array}$ \\
\hline
\end{tabular}




\section{Future Project Recommendations}

Several improvements can be made to the research performed in this paper. The first would be to improve upon the temperature studies performed in Chapter 4. The structure that was created for the experiments (Figure 4.1, pg. 49) was very rudimentary and could have potentially been the primary reason why the temperature-dependent critical voltage results in Section 4.2 were not monotomic in nature. Also, an increased pool of HEMTs and experimental temperature points would produce more reliable results. For example, instead of making gate leakage current measurements from $27^{\circ} \mathrm{C}$, $40^{\circ} \mathrm{C}-100^{\circ} \mathrm{C}$ in $10^{\circ} \mathrm{C}$ steps, it might be more advantageous to make the measurements in temperature steps of $5^{\circ} \mathrm{C}$ (even finer if the data acquisition becomes more efficient). Increasing the amount of temperature data points taken during the critical voltage experiments would also aid in the construction of Arrhenius plots, which would help identify the activation energies associated with the temperature dependence of $\mathrm{V}_{\text {crit. }}$.

Better methods and testing strategies need to be developed for the electron trapping and detrapping experiments performed in Chapter 5. While some detrapping events were observed during experimentation, the detrapping methodology needs to be redefined and improved upon. For example, work done in [39] utilizes pulsed biasing during the electron trapping and detrapping procedures, and also analyzes the time constants associated with the trapping and detrapping events. Knowledge about the time constants can explain where within the device the traps are localized (e.g. deep traps in the GaN buffer). C-V measurements may also be beneficial in this study. 
While the tuning method used to extract the small-signal parameters in Chapter 6 produced quick and rough estimates as to how the parameters were changing with respect to high-field stress, the method is by no means perfectly accurate. The tuning performed in Agilent ADS was based off of a "best-guess" methodology where-in simulated parameters were tuned until the simulated S-parameters were comparable to what was measured. A better strategy would be to investigate the mathematical models presented in papers such as [43], [44], [45], [46], and [47]. The papers discuss proper biasing schemes to extract various sets of S-parameters, which are in turn involved in Z- or Ytransformations to map the measured parameters to small-signal parameters. The methods were attempted during the RF studies performed in this paper, but the results from the mathematical models could not be made to converge.

One particular concept that would be beneficial in the investigation of GaNrelated defect mechanisms would be a thorough 1/f-noise analysis. Work performed in [48] found that higher gate leakage currents in AlGaN/GaN HEMTs contributed to reduced noise performance. For fundamental background on noise sources, refer to [49]. Preliminary research on a 1/f-noise measurement system was performed at the early stages of this research, but unfortunately the efforts could not be carried out in full. Preliminary 1/f-noise measurement system designs and simulations are presented in Appendix A: 1/f-Noise System Prototype. Because HEMTs are very attractive in power amplification applications that may involve mixers in the system design (such as in a phase-locked loop, or PLL), noise can become a major concern, especially if the noise can be upconverted to the signal of interest (leading to degraded signal to noise ratios). An interesting investigation would be to evaluate the noise performance of GaN-on-Si 
HEMTs exposed to high electric field stress and identify mechanisms that could contribute to increased noise levels (e.g. electron detrapping contributing to increased burst noise). To allow for robust noise measurements, the system would need to be encased in a PEC casing to prevent external noise sources from influencing measurements. 


\section{List of References}

[1] F. Fornetti, "Wide Bandgap Semiconductor Materials and Microwave PAs," 16 February 2012. [Online]. Available: http://explorerf.com/resources.html. [Accessed 19 January 2013].

[2] G. Simin, "High Electron Mobility Transistors (HEMTs)," University South Carolina, [Online]. Available:

www.ee.sc.edu/personal/faculty/simin/ELCT871/18\%20AlGAN-

GAN\%20HEMTs.pdf. [Accessed 19 January 2013].

[3] K. Chang, "Operation Principles of HEMTs," in Microwave Solid-State Circuits and Applications, New York, John Wiley \& Sons, Inc., 1994, p. 326.

[4] U. K. Mishra, "AlGaN/GaN HEMTs: An overview of device operation and applications," University of California, Santa Barbara, Santa Barbara.

[5] K. Brueckner and et. al., "Two-dimensional electron gas based actuation of piezoelectric AlGaN/GaN microelectromechanical resonators," Applied Physics Letters, vol. 93, 2008.

[6] T. R. Lenka and A. K. Panda, "Characteristics Study of 2DEG Transport Properties of AlGaN/GaN and AlGaAs/GaAs-based HEMT," National Institute of Science and Technology, Odisha, 2010.

[7] A. Kistchinsky, "Ultra-wideband GaN power amplifiers - from innovative technology to standard products," 2011.

[8] E. F. Schubert, "High Electron Mobility Transistors," Rensselaer Polytechnic Institute , 2003. [Online]. Available: http://www.ecse.rpi.edu/ schubert/CourseECSE-6290\%20SDM-2/1\%20HEMTs.pdf. [Accessed 19 January 2013].

[9] M. Kameche, "GaAs-, InP-, and GaN HEMT-based Microwave Control Devices: What is Best and Why," Microwave Journal, Noordwijk, 2005.

[10] J. del Alamo and J. Joh, "GaN HEMT reliability," Microelectronics Reliability, no. 49, pp. 1200-1206, 2009.

[11] S. Y. Park and et. al., "Physical degradation of GaN HEMT devices under high drain bias reliability testing," Microelectronics Reliability, 2009. 
[12] J. W. Johnson and et. al., "Material, process, and device development of GaN-based HFETs on silicon substrates," Electrochemical Society Proceedings, p. 405, 2004.

[13] Nitronex Corporation, "NPTB00004 Product Details - Data Sheet," November 2007. [Online]. Available: http://www.nitronex.com/NPTB00004_Product_Page.html. [Accessed March 2012].

[14] Nitronex Corporation, "NPTB00004 Quality and Reliability - NPTB00004 Qualification Report" May 2009. [Online]. Available: http://www.nitronex.com/NPTB00004_Product_Page.html. [Accessed September 2012].

[15] R. Thierren and et. al., "A 36mm GaN-on-Si HFET producing 368W at 60V with 70\% drain efficiency," in IEDM Technical Digest, 2005.

[16] J. W. Johnson and et. al., "12 W/mm AlGaN-GaN HFETs on silicon substrates," Electron Device Letters, vol. 25, no. 7, pp. 459 - 461, 2004.

[17] Nitronex Corporation, "GaN Essentials: AN-011 Substrates for GaN RF Devices," June 2008. [Online]. Available: http://www.nitronex.com/ganessentials.html. [Accessed 16 May 2012].

[18] J. Joh, F. Gao, T. Palacios and J. A. del Alamo, "A model for the critical voltage for electrical degradation of GaN high electron mobility transistors," Electron Device Letters, vol. 29, no. 4, pp. 287 - 289, 2008.

[19] P. Makaram, J. Joh, J. A. del Alamo, T. Palacios and C. V. Thompson, "Evolution of structural defects associated with electrical degradation in AlGaN/GaN high electron mobility transistors," Applied Physics Letters, vol. 96, no. 23, pp. 233509 233509-3, 2010.

[20] T. Ohki and et. al., "Reliability of GaN HEMTs: Current status and future technology," in 47th Annual International Reliability Physics Symposium, Montreal, 2009.

[21] Nitronex Corporation, “Application Notes - AN-009," October 2008. [Online]. Available: http://www.nitronex.com/application_notes.html. [Accessed 1 May 2012].

[22] C. K. Alexander and M. N. Sadiku, "Step Response of an RC Circuit," in Fundamentals of Electric Circuits, New York, McGraw-Hill Companies, Inc., 2007, p. 275. 
[23] P. Burgaud, L. Constancias, G. Martel, C. Savina and D. Mesnager, "Preliminary reliability assessment and failure physical analysis on AlGaN/GaN HEMTs COTS," Microelectronics Reliability, vol. 47, pp. 1653-1657, 2007.

[24] F. Li-Hua and et. al., "High-field induced electron detrapping in AlGaN/GaN high electron mobility transistor," Chinese Physics B, vol. 21, no. 10, 2012.

[25] S. Demirtas, J. Joh and J. A. del Alamo, "High voltage degradation of GaN high electron mobility transistors on silicon substrate," Microelectronics Reliability, no. 50, pp. 758-762, 2010.

[26] C.-T. Chang and et. al., "Change of electrical characteristics for AlGaN/GaN HEMTs under uniaxial tensile strain," IEEE Electron Device Letters, vol. 20, no. 7 , 2009.

[27] V. Gavryushin and A. Zukauskas, "The Metal-Semiconductor Junction: Schottky Diode," 2002. [Online]. Available:

http://www.pfk.ff.vu.lt/lectures/funkc_dariniai/diod/schottky.htm. [Accessed 1912 2012].

[28] C.-F. Lo and et. al., "Improved off-state stress critical voltage on AlGaN/GaN high electron mobility transistors utilizing Pt/Ti/Au based gate metallization," ECS Transactions, vol. 41, no. 6, pp. 63-70, 2011.

[29] Y. Cai, Y. Zhou, K. M. Lau and K. J. Chen, "Control of threshold voltage of AlGaN/GaN HEMTs by fluoride-based plasma treatment: from depletion mode to enhancement mode," IEEE Transcations on Electron Devices, vol. 53, no. 9, pp. 2207-2215, 2006.

[30] G. Gonzalez, "Characteristics of Microwave Transistors," in Microwave Transistor Amplifiers: Analysis and Design, New Jersey, Prentice-Hall, Inc., 1997, pp. 79-86.

[31] University of Conneticut, "Lecture 17 - Dielectrics and Ferroelectrics," [Online]. Available: http://www.ims.uconn.edu/ alpay/Courses/MMAT\%20244/. [Accessed 2812 2012].

[32] Nitronex Corporation, "GaN Essentials: AN-012 - Thermal Considerations for GaN Technology," June 2008. [Online]. Available: http://www.nitronex.com/ganessentials.html. [Accessed 1 May 2012].

[33] Y. Li-Yuan and et. al, "High temperature characteristics of AlGaN/GaN high electron mobility transistors," Chinese Physics B, vol. 20, no. 11, 2011. 
[34] Y. C. Chou and et. al., "Degradation of AlGaN/GaN HEMTs under elevated temperature lifetesting," Microelectronics Reliability, vol. 44, pp. 1033-1038, 2004.

[35] B. A. Polash, M. A. Huque, S. K. Islam and H. Huq, "High temperature performance measurement and analysis of GaN HEMTs," in Proceedings of SPIE, 2008.

[36] A. M. Darwish, A. A. Ibrahim and H. A. Hung, "Temperature dependence of GaN HEMT small signal parameters," International Journal of Microwave Science and Technology, 2011.

[37] E. A. Douglas and et. al., "Investigation of the effect of temperature during off-state degradation of $\mathrm{AlGaN} / \mathrm{GaN}$ high electron mobility transistors," Microelectronics Reliability, vol. 52, pp. 23-28, 2012.

[38] J. Joh and J. A. del Alamo, "Effects of temperature on electrical degradation of GaN high electron mobility transistors," in ICNS Proceedings, 2007.

[39] J. Joh and J. A. del Alamo, "A current-transient methodology for trap analysis for GaN high electron mobility transistors," IEEE Transactions on Electron Devices, vol. 58, no. 1, 2011.

[40] S. DasGupta and et. al., "Slow detrapping transients due to gate and drain bias stress in high breakdown voltage AlGaN/GaN HEMTs," IEEE Transactions on Electron Devices , vol. 59, no. 8, pp. 2115-2122, 2012.

[41] Miller Technology, Inc., "Technical Notes and Information - The Series to Parallel Impedance Transformation," [Online]. Available:

http://www.millertechinc.com/pdf_files/TN113\%20Series\%20to\%20Paralle1\%20Im pedance\%20Transformation.pdf. [Accessed 23 May 2012].

[42] C. K. Alexander and M. N. Sadiku, "Capacitors," in Fundamentals of Electric Circuits, New York, McGraw-Hill, 2007, p. 216.

[43] M. Berroth and R. Bosch, "High-frequency equivalent circuit of GaAs FET's for large-signal applications," IEEE Transactions on Microwave Theory and Techniques, vol. 39, no. 2, 1991.

[44] E. Chigaeva and et. al., "Determination of small-signal parameters of GaN-based HEMTs," IEEE, 2000.

[45] D. Maafri, M. C. Yagoub and R. Touhami, "A new small-signal modeling and extraction method in AlGaN/GaN HEMTs," International Journal of Research and Reviews in Information Sciencies (IJRRIS), vol. 2, no. 1, pp. 150-154, 2012. 
[46] A. Jarndal and G. Kompa, "A new small signal model parameter extraction method applied to GaN devices," IEEE, 2005.

[47] G. Chen, V. Kumar, R. S. Schwindt and I. Adesida, "A low gate bias model extraction technique for AlGaN/GaN HEMTs," IEEE Transactions on Microwave Theory and Techniques, vol. 54, no. 7, pp. 2949-2953, 2006.

[48] S. L. Rumyantsev and et. al., "Effect of gate leakage current on noise properties of AlGaN/GaN field effect transistors," Journal of Applied Physics, vol. 88, no. 11, 2000.

[49] F. N. Hooge, "1/f noise sources," IEEE Transcations on Electron Devices, vol. 41, no. 11, pp. 1926-1935, 1994.

[50] C. Toro, "Improved 1/f noise measurements for microwave transistors," University of South Florida, Tampa, 2004.

[51] K. Kundert, “The Designer's Guide,” 2006. [Online]. Available: http://www.designers-guide.org/Design/bypassing.pdf. [Accessed 15 May 2012].

[52] F. Seifert, "Resistor Current Noise Measurements".

[53] D. T. Smith, "Low Frequency Noise in Tantalum Capacitors," Active and Passive Electronic Components, vol. 12, pp. 215-221, 1987. 


\section{Appendices}

\section{Appendix A: 1/f-Noise System Prototype}

An improved 1/f noise measurement system was proposed by Clemente Toro, a post-graduate student from the University of South Florida [50]. Toro's thesis involved developing a general 1/f noise system that could provide broad noise analyses from $1 \mathrm{~Hz}$ to $10 \mathrm{MHz}$ for a number of microwave transistors (BJTs, FETs, HBTs, HEMTs, etc.). Figure A.1 shows Toro's arbitrary noise system configuration.

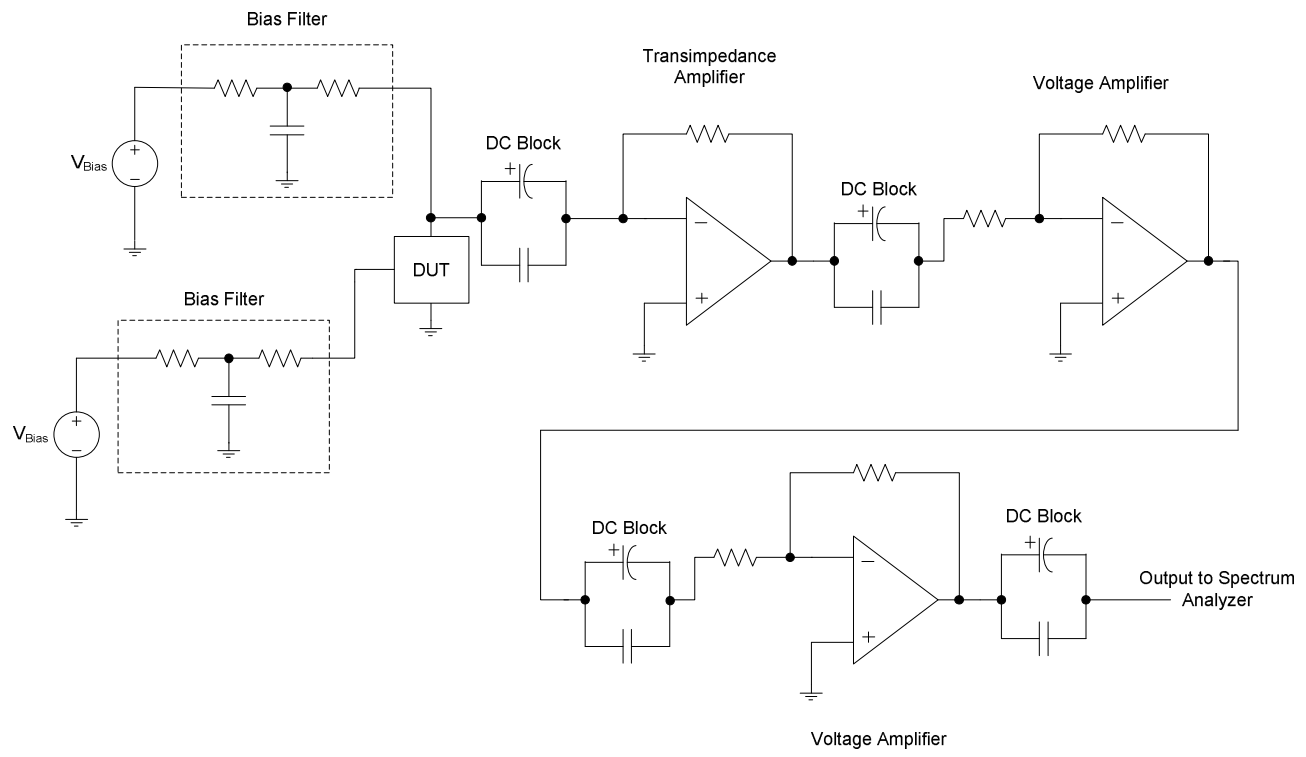

Figure A.1 $\square$ 1/f-noise measurements system for microwave transistors proposed by Clemente Toro.

The device under test used in the design of the system was the Nitronex NPTB00004 GaN-on-Si HEMT. While flicker noise exists at both the drain and the gate of the device, the prototype was only configured to make measurements from the drain terminal for proof-of-concept purposes. Each proceeding section in this paper will outline the general purpose of each stage followed by a design analysis. 


\section{Bias Filter Stages}

A majority of power supplies produce relatively noisy DC signals, which can directly affect the signal-to-noise ratio during 1/f measurements [50], [51]. Each of the bias filters were designed to provide sufficient suppression of signals greater than or equal to $1 \mathrm{~Hz}$. The recommended supply filters by Toro for a FET are shown in Figure A.2 and Figure A.3.

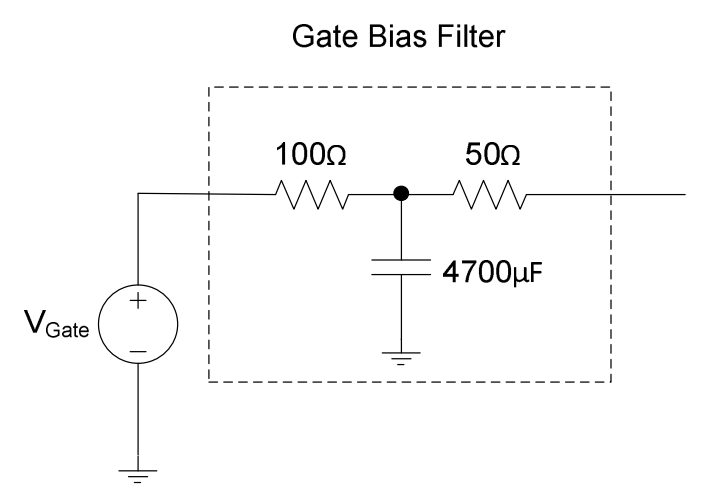

Figure A.2 ? Gate supply filter for a FET.

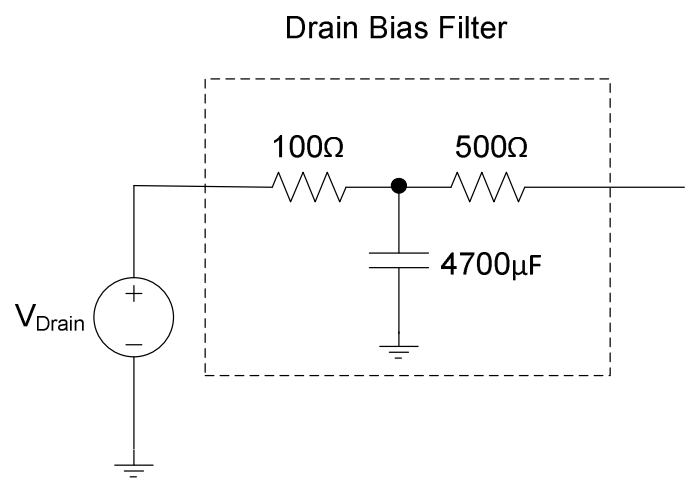

Figure A.3 ? Drain supply filter for a FET.

While Toro's design suggestions are practical for arbitrary FETs, the supply filter for the NPTB00004 must be designed with some parameters in mind. The AN-009 application note provided by Nitronex discusses the need for a $200 \Omega$ resistor along the 
gate bias feed path to dampen oscillations caused by the device [21]. If the filter of Figure 2 is used, the DC signal would only see $150 \Omega$ in the path (the capacitor to ground is an open-circuit to DC). By replacing the $50 \Omega$ resistor with a $100 \Omega$ resistor, the $200 \Omega$ value can be achieved. The final circuit design is shown in Figure A.4 and the Agilent frequency response simulation is shown in Figure A.5. The simulation was performed assuming a $50 \Omega$ system.

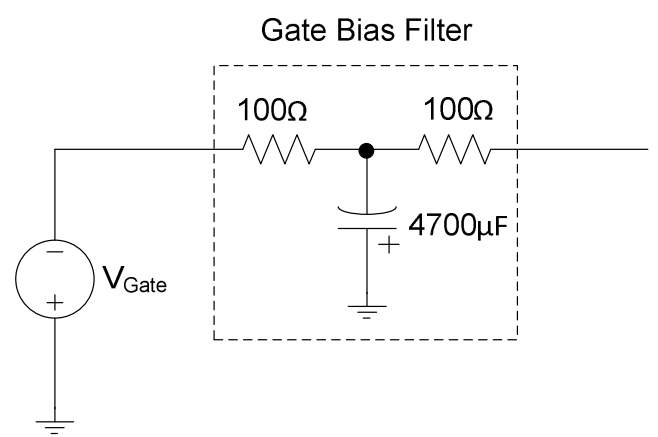

Figure A.4 ? Gate supply filter for the NPTB00004 HEMT.

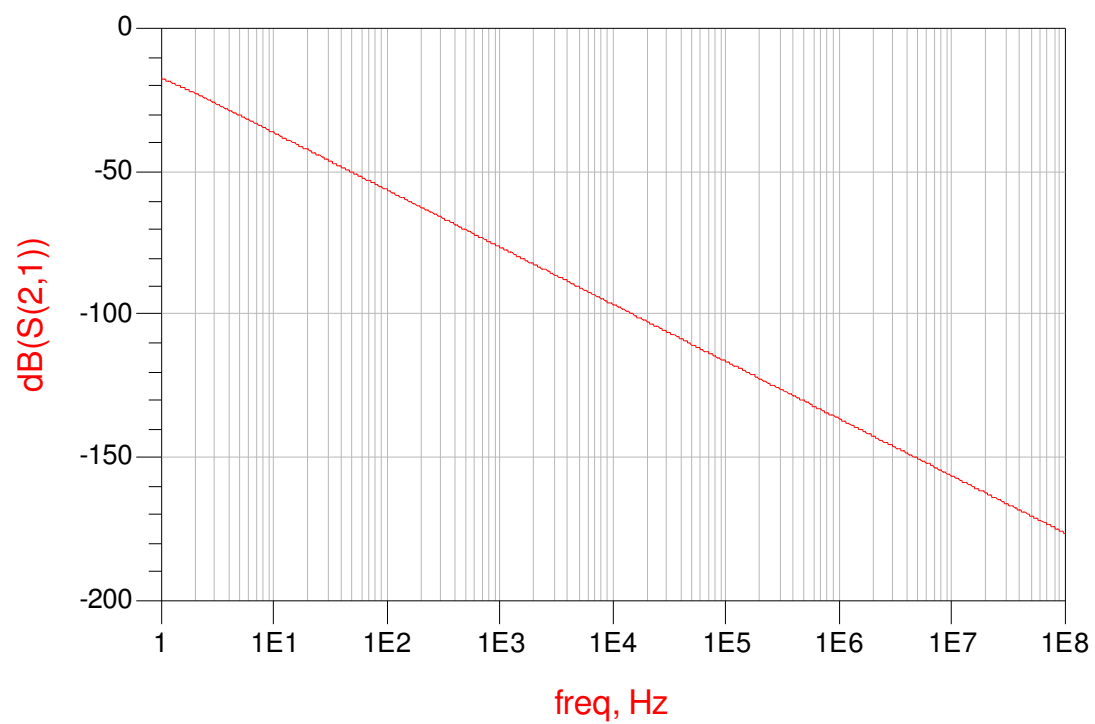

Figure A.5 ? Gate supply filter frequency response. 
The drain supply filter of Figure A.3 was designed for arbitrary FETs, where low supply voltage was used. The GaN HEMT, however, is a high-powered device where nominal power dissipation can be anywhere from $2-3 \mathrm{~W}$. Therefore, high current draw will flow through the drain feed path depending on the value of $V_{g s}$ and $V_{d s}$ (the NPTB00004 is a depletion-mode device, so $\mathrm{V}_{\mathrm{gs}}$ controls the depletion region of the device). With high current draw and resistive elements in the feed path, large voltage drops will be present in the circuit of Figure A.3.

To remove the voltage drops, the filter could be redesigned by removing the resistors and placing several capacitors in parallel along the supply line to filter a broad range of AC signals (large capacitors filter low frequencies, while small capacitors filter high frequencies). There are two reasons why the two series resistors are needed, however. The first reason is that the $500 \Omega$ resistor provides a high impedance to the $1 / \mathrm{f}$-noise at the drain of the device. If the $500 \Omega$ resistor is removed, the $1 / \mathrm{f}$ noise could potentially see an AC short to ground via the feed line capacitor instead of taking the path through the transimpedance amplifier. Toro suggests keeping the $500 \Omega$ resistor as the minimum-valued resistor required to prevent the $1 / \mathrm{f}$ noise from feeding back into the supply filter. The $100 \Omega$ resistor is required to aid in dampening oscillatory effects in the event that the $4700 \mu \mathrm{F}$ capacitor resonates with the inherent inductance of the supply.

The drain supply was simulated using Agilent ADS. The first simulation utilizes the circuit configuration of Figure A.3. To provide better power distribution along the feed path, two $200 \Omega$ resistors were placed in parallel to form the $100 \Omega$ resistor, while two $1 \mathrm{k} \Omega$ resistors were placed in parallel to achieve the $500 \Omega$ resistor. Figure A.6 shows the 
NPTB00004 ADS model in a DC configuration. Table A.1 and Figure A.7 show the resulting DC characteristics and frequency response, respectively.

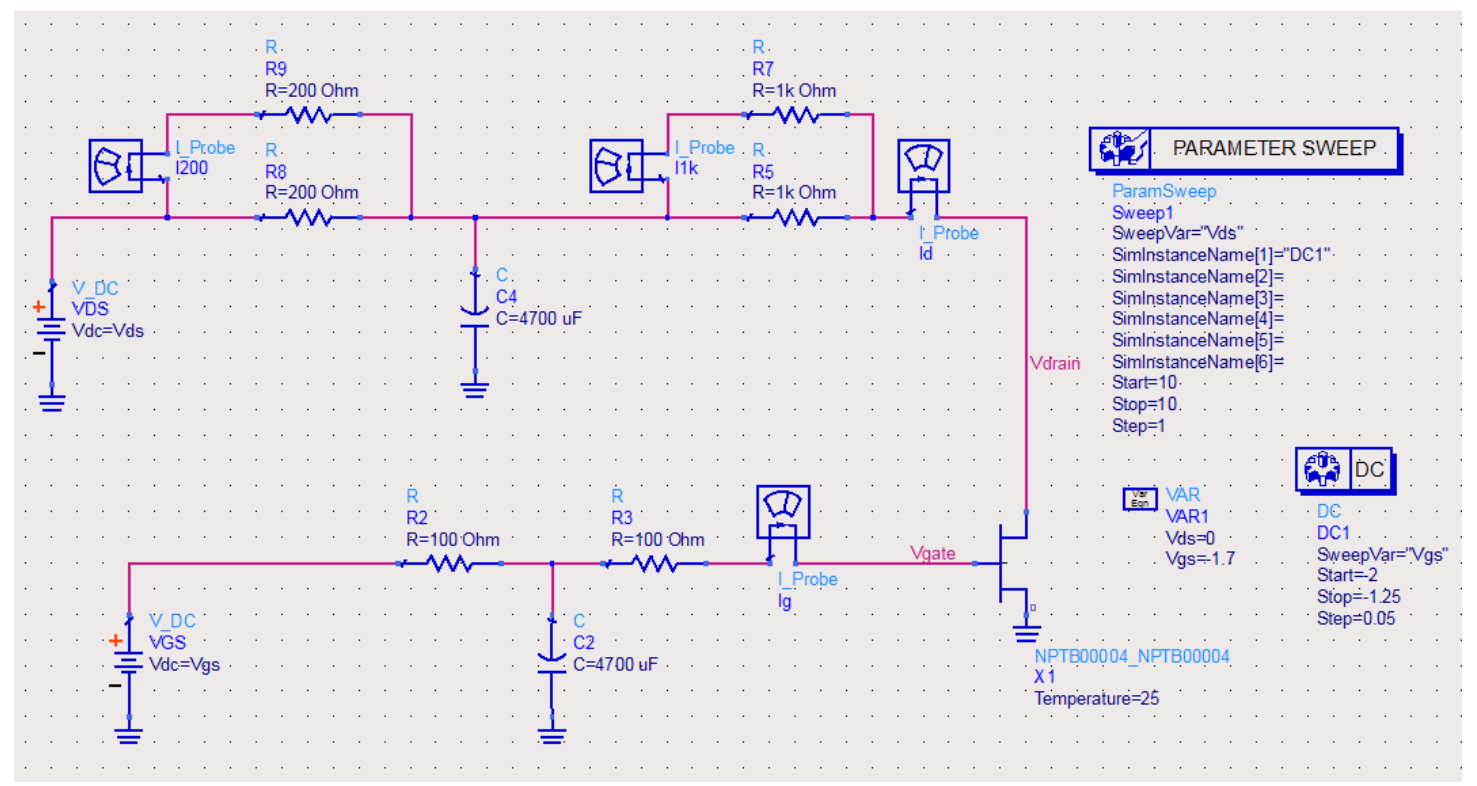

Figure A.6 ? DC circuit simulation setup for the NPTB00004.

Table A.1 ? NPTB00004 DC characteristics using Toro's drain supply filter design (with parallel resistors).

\begin{tabular}{|r|r|r|r|r|}
\hline \multicolumn{1}{|c|}{ Vgs } & \multicolumn{1}{|c|}{ ld.i } & \multicolumn{1}{|c|}{ Vdrain } & \multicolumn{1}{|c|}{ l1k.i } & \multicolumn{1}{c|}{ l200.i } \\
\cline { 2 - 4 } & Vds=10.000 & Vds=10.000 & Vds=10.000 & Vds=10.000 \\
\hline-2.000 & $2.169 \mathrm{~mA}$ & $8.699 \mathrm{~V}$ & $1.084 \mathrm{~mA}$ & $1.084 \mathrm{~mA}$ \\
-1.950 & $2.882 \mathrm{~mA}$ & $8.271 \mathrm{~V}$ & $1.441 \mathrm{~mA}$ & $1.441 \mathrm{~mA}$ \\
-1.900 & $3.787 \mathrm{~mA}$ & $7.728 \mathrm{~V}$ & $1.894 \mathrm{~mA}$ & $1.894 \mathrm{~mA}$ \\
-1.850 & $4.921 \mathrm{~mA}$ & $7.047 \mathrm{~V}$ & $2.460 \mathrm{~mA}$ & $2.460 \mathrm{~mA}$ \\
-1.800 & $6.324 \mathrm{~mA}$ & $6.206 \mathrm{~V}$ & $3.162 \mathrm{~mA}$ & $3.162 \mathrm{~mA}$ \\
-1.750 & $8.043 \mathrm{~mA}$ & $5.174 \mathrm{~V}$ & $4.022 \mathrm{~mA}$ & $4.022 \mathrm{~mA}$ \\
-1.700 & $10.14 \mathrm{~mA}$ & $3.917 \mathrm{~V}$ & $5.069 \mathrm{~mA}$ & $5.069 \mathrm{~mA}$ \\
-1.650 & $12.69 \mathrm{~mA}$ & $2.389 \mathrm{~V}$ & $6.343 \mathrm{~mA}$ & $6.343 \mathrm{~mA}$ \\
-1.600 & $15.46 \mathrm{~mA}$ & $723.3 \mathrm{mV}$ & $7.731 \mathrm{~mA}$ & $7.731 \mathrm{~mA}$ \\
-1.550 & $16.00 \mathrm{~mA}$ & $398.3 \mathrm{mV}$ & $8.001 \mathrm{~mA}$ & $8.001 \mathrm{~mA}$ \\
-1.500 & $16.17 \mathrm{~mA}$ & $296.8 \mathrm{mV}$ & $8.086 \mathrm{~mA}$ & $8.086 \mathrm{~mA}$ \\
-1.450 & $16.27 \mathrm{~mA}$ & $240.3 \mathrm{mV}$ & $8.133 \mathrm{~mA}$ & $8.133 \mathrm{~mA}$ \\
-1.400 & $16.33 \mathrm{~mA}$ & $203.2 \mathrm{mV}$ & $8.164 \mathrm{~mA}$ & $8.164 \mathrm{~mA}$ \\
-1.350 & $16.37 \mathrm{~mA}$ & $176.9 \mathrm{mV}$ & $8.186 \mathrm{~mA}$ & $8.186 \mathrm{~mA}$ \\
-1.300 & $16.40 \mathrm{~mA}$ & $157.4 \mathrm{mV}$ & $8.202 \mathrm{~mA}$ & $8.202 \mathrm{~mA}$ \\
-1.250 & $16.43 \mathrm{~mA}$ & $142.4 \mathrm{mV}$ & $8.215 \mathrm{~mA}$ & $8.215 \mathrm{~mA}$ \\
& & & \\
\hline
\end{tabular}




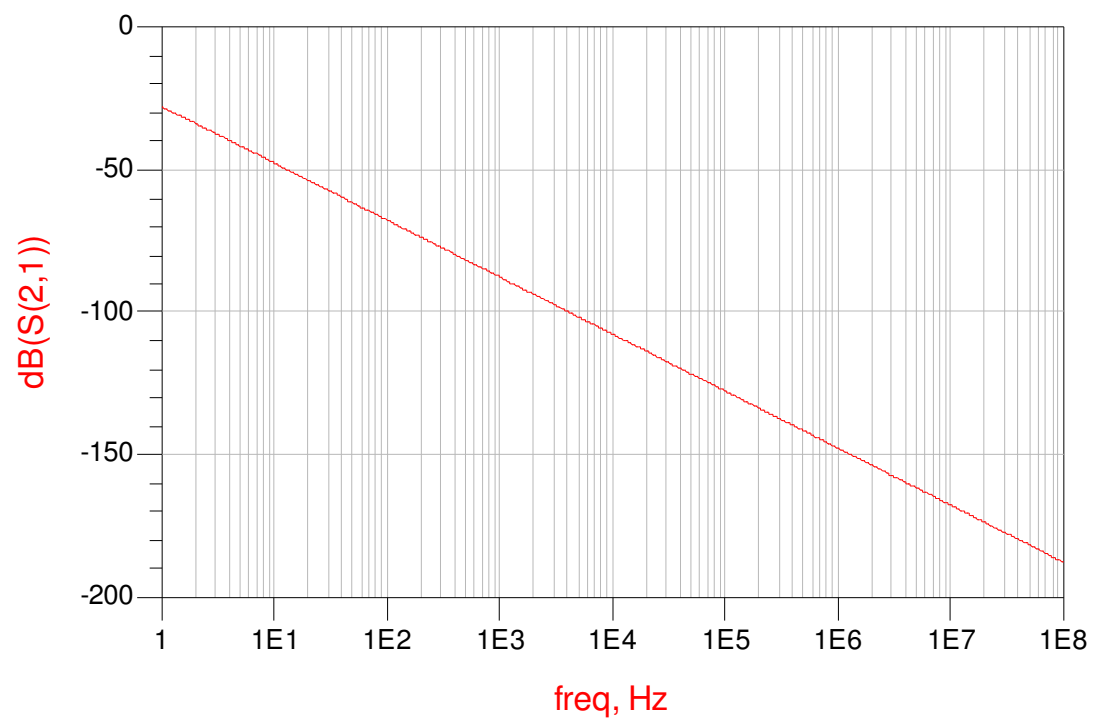

Figure A.7 [ Drain supply filter frequency response.

As seen in Table A.1, after setting $\mathrm{V}_{\mathrm{ds}}$ to $10 \mathrm{~V}$ and stepping $\mathrm{V}_{\mathrm{gs}}$ more positive, the drain current starts to increase and the effective drain voltage at the device starts to diminish. In other words, the resistive elements alter the quiescent point of the HEMT. The aforementioned effect is shown graphically in Figure A.8.

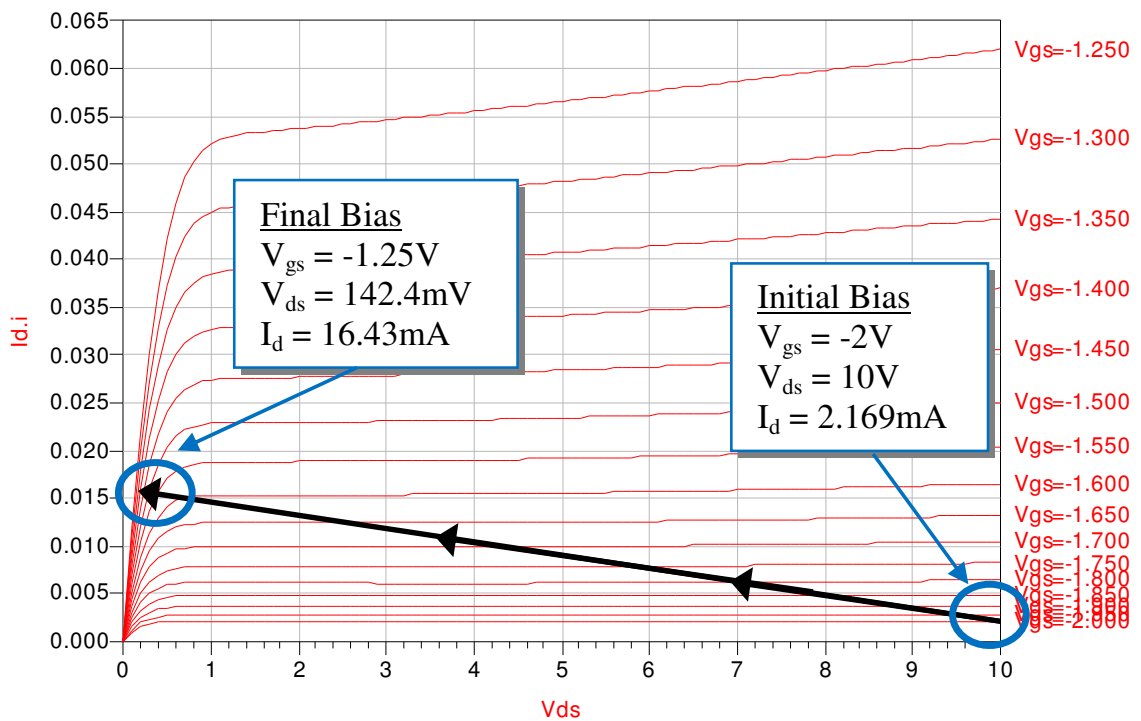

Figure A.8 Q Q-point alteration due to resistive components in the drain supply filter. 
Next, the $200 \Omega$ parallel pair was replaced with a $10 \Omega$ resistor. While the $10 \Omega$ resistor provides subtle dampening effects in comparison to the effective $100 \Omega$ resistor, the power dissipation and voltage drop would be smaller. In the event that the $4700 \mu \mathrm{F}$ parallel resonates with the inherent inductance of the supply, the $10 \Omega$ would still aid in lowering the Q-factor of the resonant components (as seen from a series to parallel transformation in [41]). Table A.2 and Figure A.9 show the DC characteristics and frequency response using the altered filter configuration, respectively. While the Q-point shift is similar to the effects seen in Figure A.8, the overall drain current to the device is higher and the voltage at the drain of the device is also higher. The frequency response of the redesigned filter is also similar to that of the original design.

Table A.2 ? NPTB00004 DC characteristics using the $10 \Omega$ resistor in the drain filter.

\begin{tabular}{|r|r|r|r|}
\hline \multirow{2}{*}{ Vgs } & \multicolumn{1}{|c|}{ Id.i } & Vdrain & \multicolumn{1}{|c|}{ l1k.i } \\
\cline { 2 - 4 } & Vds=10.000 & Vds=10.000 & Vds=10.000 \\
\hline-2.000 & $2.173 \mathrm{~mA}$ & $8.892 \mathrm{~V}$ & $1.086 \mathrm{~mA}$ \\
-1.950 & $2.890 \mathrm{~mA}$ & $8.526 \mathrm{~V}$ & $1.445 \mathrm{~mA}$ \\
-1.900 & $3.800 \mathrm{~mA}$ & $8.062 \mathrm{~V}$ & $1.900 \mathrm{~mA}$ \\
-1.850 & $4.940 \mathrm{~mA}$ & $7.481 \mathrm{~V}$ & $2.470 \mathrm{~mA}$ \\
-1.800 & $6.352 \mathrm{~mA}$ & $6.761 \mathrm{~V}$ & $3.176 \mathrm{~mA}$ \\
-1.750 & $8.079 \mathrm{~mA}$ & $5.879 \mathrm{~V}$ & $4.040 \mathrm{~mA}$ \\
-1.700 & $10.17 \mathrm{~mA}$ & $4.811 \mathrm{~V}$ & $6.087 \mathrm{~mA}$ \\
-1.650 & $12.70 \mathrm{~mA}$ & $3.525 \mathrm{~V}$ & $7.863 \mathrm{~mA}$ \\
-1.600 & $15.73 \mathrm{~mA}$ & $1.980 \mathrm{~V}$ & $9.198 \mathrm{~mA}$ \\
-1.550 & $18.40 \mathrm{~mA}$ & $618.4 \mathrm{mV}$ & $9.419 \mathrm{~mA}$ \\
-1.500 & $18.84 \mathrm{~mA}$ & $392.2 \mathrm{mV}$ & $9.507 \mathrm{~mA}$ \\
-1.450 & $19.01 \mathrm{~mA}$ & $302.7 \mathrm{mV}$ & $9.559 \mathrm{~mA}$ \\
-1.400 & $19.12 \mathrm{~mA}$ & $250.2 \mathrm{mV}$ & $9.593 \mathrm{~mA}$ \\
-1.350 & $19.19 \mathrm{~mA}$ & $214.9 \mathrm{mV}$ & $9.618 \mathrm{~mA}$ \\
-1.300 & $19.24 \mathrm{~mA}$ & $189.5 \mathrm{mV}$ & $9.637 \mathrm{~mA}$ \\
-1.250 & $19.27 \mathrm{~mA}$ & $170.4 \mathrm{mV}$ & \\
\hline
\end{tabular}




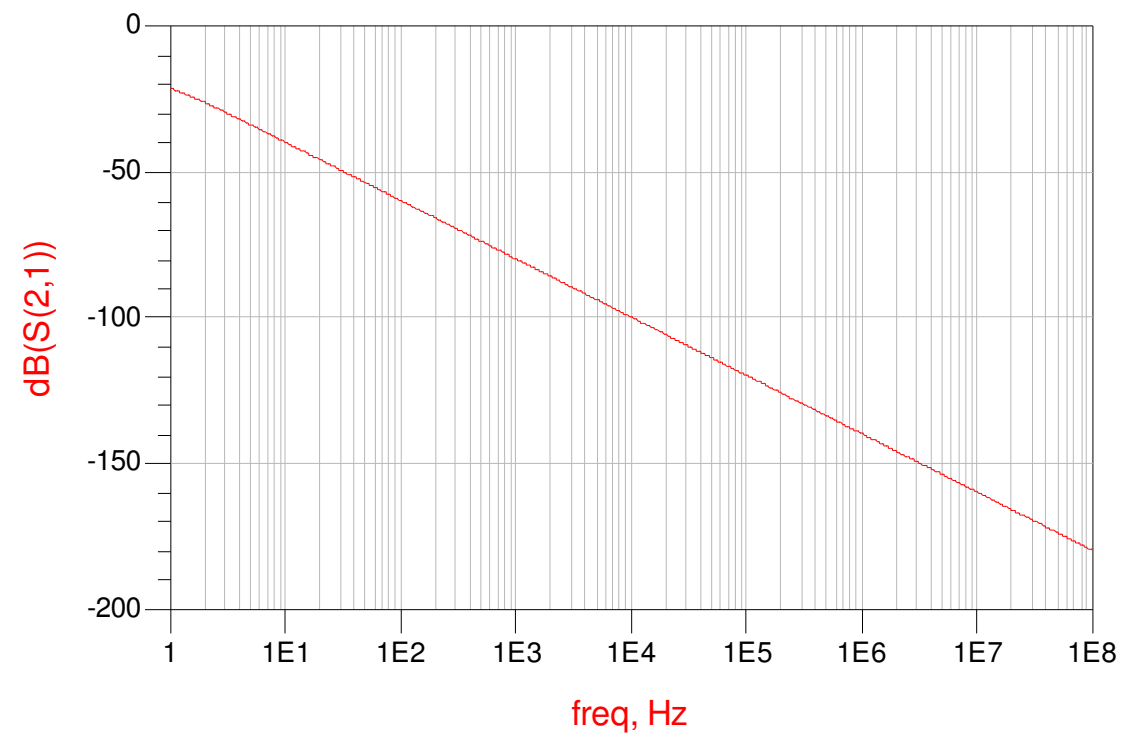

Figure A.9 ? Frequency response using the $10 \Omega$ resistor in the drain filter.

Another design strategy involves replacing the $1 \mathrm{k} \Omega$ parallel pair with a diode. The diode would not only improve upon the unwanted voltage drops and allow for a more accurate biasing of the Q-point, but would also restrict noise current from filtering through the $4700 \mu \mathrm{F}$ capacitor. The main trade-off with using a diode is that the component is a semiconductor material, which will contribute noise $\mathrm{c}$ to the overall spectral response. The DC characteristics and frequency response using a diode are seen in Table A.3 and Figure A.10, respectively. The last two columns in Table A.3 were used to determine the voltage drop across the diode during the sweep. 
Table A.3 [ NPTB00004 DC characteristics using the diode in the drain filter.

\begin{tabular}{|r|r|r|r|r|}
\hline \multicolumn{1}{|c|}{ Vgs } & \multicolumn{1}{|c|}{ Id.i } & Vdrain & var("VD+") & \multicolumn{1}{c|}{ var("VD-") } \\
\cline { 2 - 4 } & Vds=10.000 & Vds=10.000 & Vds=10.000 & Vds=10.000 \\
\hline-2.000 & $2.178 \mathrm{~mA}$ & $9.112 \mathrm{~V}$ & $9.978 \mathrm{~V}$ & $9.112 \mathrm{~V}$ \\
-1.950 & $2.907 \mathrm{~mA}$ & $9.091 \mathrm{~V}$ & $9.971 \mathrm{~V}$ & $9.091 \mathrm{~V}$ \\
-1.900 & $3.840 \mathrm{~mA}$ & $9.067 \mathrm{~V}$ & $9.962 \mathrm{~V}$ & $9.067 \mathrm{~V}$ \\
-1.850 & $5.020 \mathrm{~mA}$ & $9.042 \mathrm{~V}$ & $9.950 \mathrm{~V}$ & $9.042 \mathrm{~V}$ \\
-1.800 & $6.499 \mathrm{~mA}$ & $9.014 \mathrm{~V}$ & $9.935 \mathrm{~V}$ & $9.014 \mathrm{~V}$ \\
-1.750 & $8.333 \mathrm{~mA}$ & $8.983 \mathrm{~V}$ & $9.917 \mathrm{~V}$ & $8.983 \mathrm{~V}$ \\
-1.700 & $10.58 \mathrm{~mA}$ & $8.948 \mathrm{~V}$ & $9.894 \mathrm{~V}$ & $8.948 \mathrm{~V}$ \\
-1.650 & $13.32 \mathrm{~mA}$ & $8.909 \mathrm{~V}$ & $9.867 \mathrm{~V}$ & $8.865 \mathrm{~V}$ \\
-1.600 & $16.62 \mathrm{~mA}$ & $8.865 \mathrm{~V}$ & $9.834 \mathrm{~V}$ & $8.814 \mathrm{~V}$ \\
-1.550 & $20.55 \mathrm{~mA}$ & $8.814 \mathrm{~V}$ & $9.795 \mathrm{~V}$ & $8.757 \mathrm{~V}$ \\
-1.500 & $25.19 \mathrm{~mA}$ & $8.757 \mathrm{~V}$ & $9.748 \mathrm{~V}$ & $8.693 \mathrm{~V}$ \\
-1.450 & $30.61 \mathrm{~mA}$ & $8.693 \mathrm{~V}$ & $9.694 \mathrm{~V}$ & $8.539 \mathrm{~V}$ \\
-1.400 & $36.89 \mathrm{~mA}$ & $8.620 \mathrm{~V}$ & $9.631 \mathrm{~V}$ & $8.448 \mathrm{~V}$ \\
-1.350 & $44.08 \mathrm{~mA}$ & $8.539 \mathrm{~V}$ & $9.559 \mathrm{~V}$ & $8.348 \mathrm{~V}$ \\
-1.300 & $52.22 \mathrm{~mA}$ & $8.448 \mathrm{~V}$ & $9.478 \mathrm{~V}$ & \\
-1.250 & $61.36 \mathrm{~mA}$ & $8.348 \mathrm{~V}$ & $9.386 \mathrm{~V}$ & \\
& & &
\end{tabular}

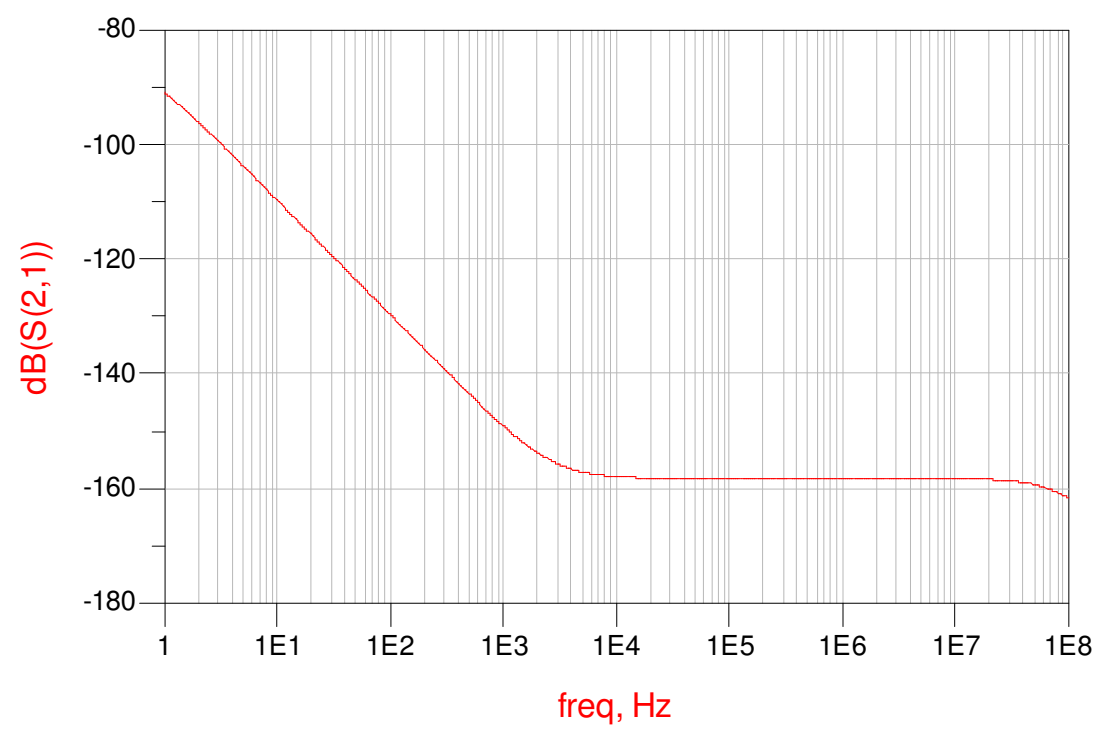

Figure A.10 ? Frequency response using the diode in the drain filter.

From Table A.3 and Figure A.10, not only do the DC characteristics improve drastically, but the filtering response also improves at lower frequencies. The final drain supply filter configuration is shown in Figure A.11. Because no more than $100 \mathrm{~mA}$ of drain current is expected through the drain path, the power rating for the $10 \Omega$ resistor is negligible (100mW worse case). A $1 \mathrm{~N} 4001$ was chosen for its high power rating of $3 \mathrm{~W}$. 
The voltage rating for the $4700 \mu \mathrm{F}$ electrolytic capacitor is also imperative to the design. A voltage rating of $25 \mathrm{~V}$ was chosen for the electrolytic.

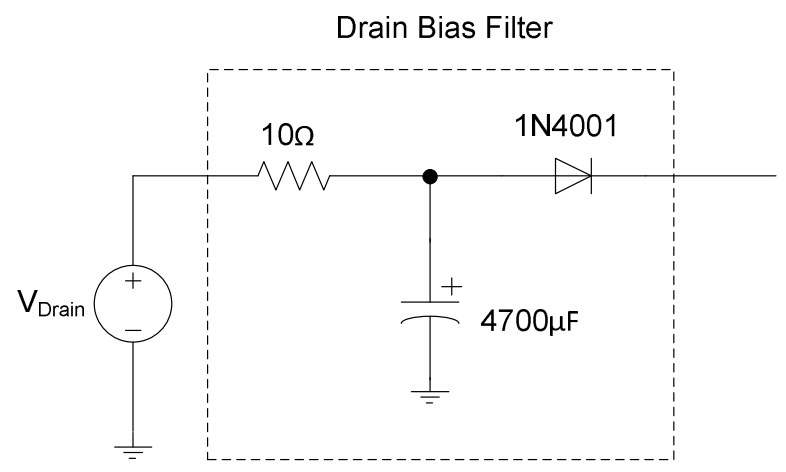

Figure A.11 ? Drain supply filter design.

\section{Transimpedance Amplifier Stage}

A transimpedance amplifier (TIA) was required to convert the noise current created by the drain into a noise voltage. Conversion of the drain noise current to a noise voltage allows for the amplification of the noise voltage using cascaded voltage amplifier stages. Design of the TIA requires the use of a low-noise operational amplifier with a large gain-bandwidth product. The low-noise specification is imperative to the design of the TIA (as well as the voltage amplifiers) because the flicker noise could potentially be mixed-in with or dominated by the noise generated by the op-amp. The gain-bandwidth product (GBP) specification must also be considered due to the general rule of thumb that the GBP of an op-amp must be at least an order or magnitude larger than the closed-loop gain multiplied by the maximum signal frequency input into the system.

The LT6200-5 was chosen for its GBP of $800 \mathrm{MHz}$ (500MHz worst-case). Knowing that the system needs to work up to $10 \mathrm{MHz}$ and the voltage amplifiers provide a gain of $5 \mathrm{~V} / \mathrm{V}$, the GBP specification for the LT6200-5 was sufficient. The TIA design using the LT6200-5 is shown in Figure A.12, which is Toro's suggested design. Using 
$100 \Omega$ as feedback provides a TIA sensitivity of 100V/A. Figure 13 shows and example of the TIA operating with the $100 \mathrm{~V} / \mathrm{A}$ configuration.

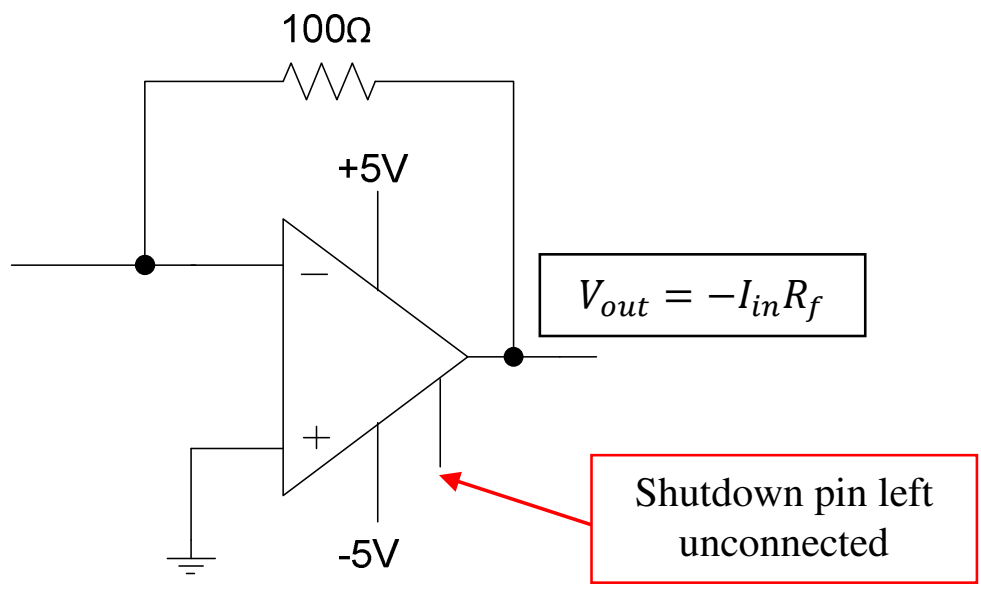

Figure A.12 ? LT6200-5 transimpedance amplifier with a sensitivity of 100V/A.

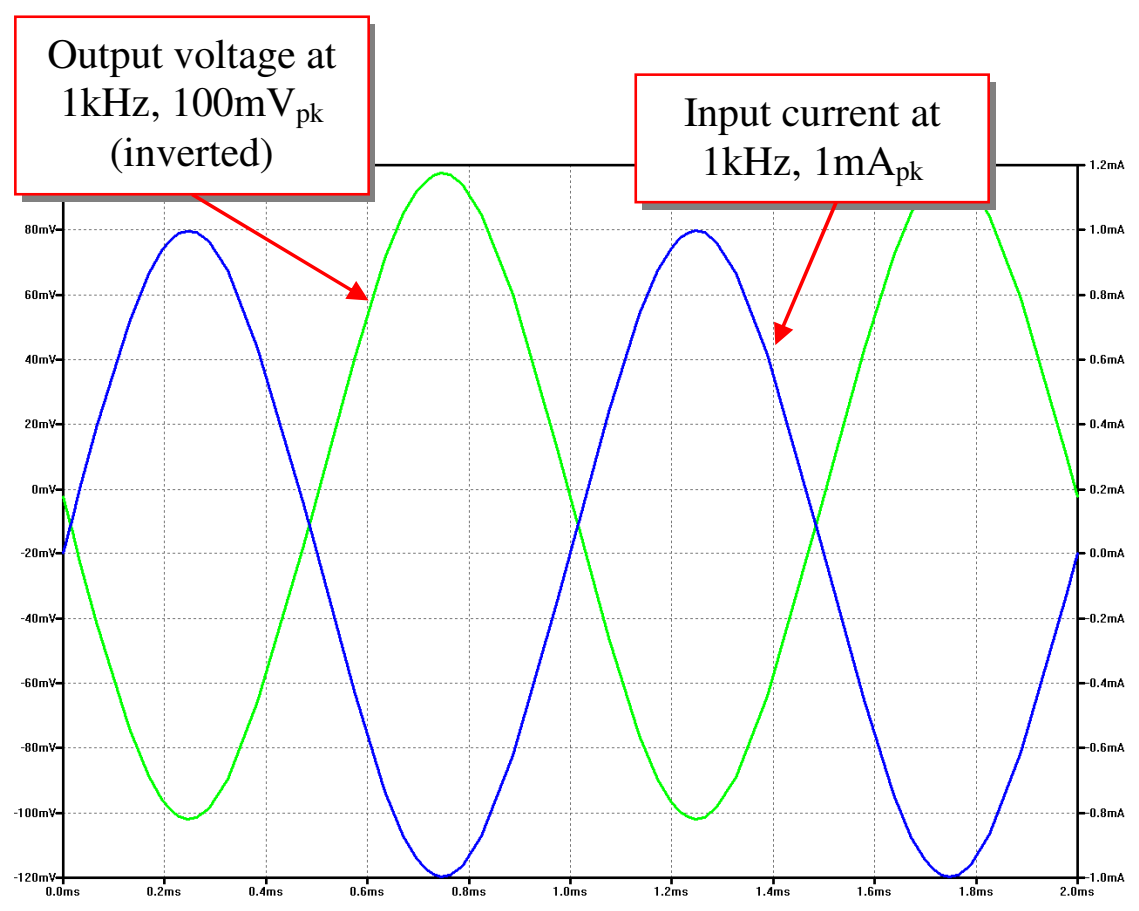

Figure A.13 ? LT6200-5 current to voltage conversion (100V/A sensitivity, voltage left-axis, current right-axis). 


\section{Voltage Amplifier Stages}

Toro designs both inverting amplifier stages to provide $14 \mathrm{~dB}(5 \mathrm{~V} / \mathrm{V})$ of amplification, resulting in $28 \mathrm{~dB}(25.12 \mathrm{~V} / \mathrm{V})$ of total amplification. Toro's suggested design for a single amplifier stage is shown in Figure A.14.

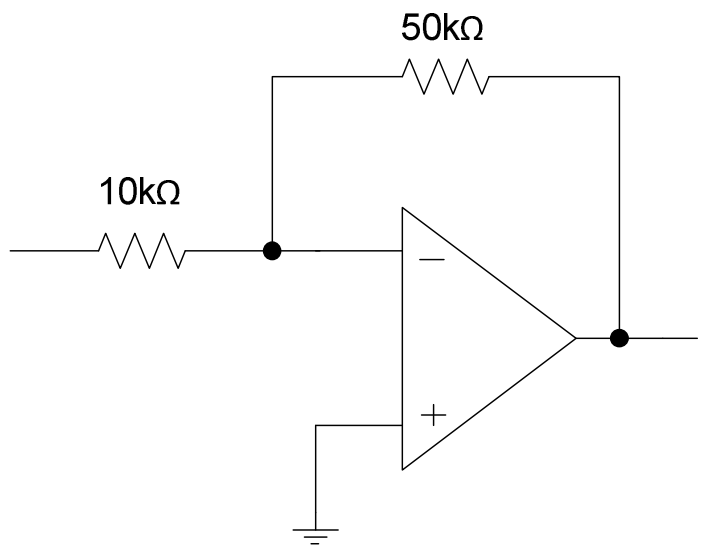

Figure A.14 ? Voltage amplifier with a gain of 14dB (5V/V).

After simulating the design using an LT6200-5 op-amp, observable peaking was found in the response (Figure A.15).To compensate the amplifier, capacitors ranging from $1 \mathrm{pF}$ up to $10 \mathrm{pF}$ were iteratively placed in parallel with the $50 \mathrm{k} \Omega$ to reduce the peaking seen near the cut-off frequency of the amplifier (adds a pole). The capacitors eliminated the peaking effect, but decreased the cut-off frequency of the amplifier. The amplifier was originally designed to provide $14 \mathrm{~dB}$ of gain from $1 \mathrm{~Hz}$ up to $10 \mathrm{MHz}$. Toro used a $3-\mathrm{dB}$ frequency of $17 \mathrm{MHz}(11 \mathrm{~dB})$ as a figure of merit in his design.

To provide sufficient gain up to $10 \mathrm{MHz}$, the $10 \mathrm{k} \Omega$ and $50 \mathrm{k} \Omega$ resistors were scaled down to $1 \mathrm{k} \Omega$ and $5 \mathrm{k} \Omega$, respectively. The parallel feedback capacitor was also swept between $1 \mathrm{pF}$ to $10 \mathrm{pF}$ during LTSpice simulations. The LTSPice simulated circuit is shown in Figure A.16 and the resulting responses are shown in Figure A.17. 


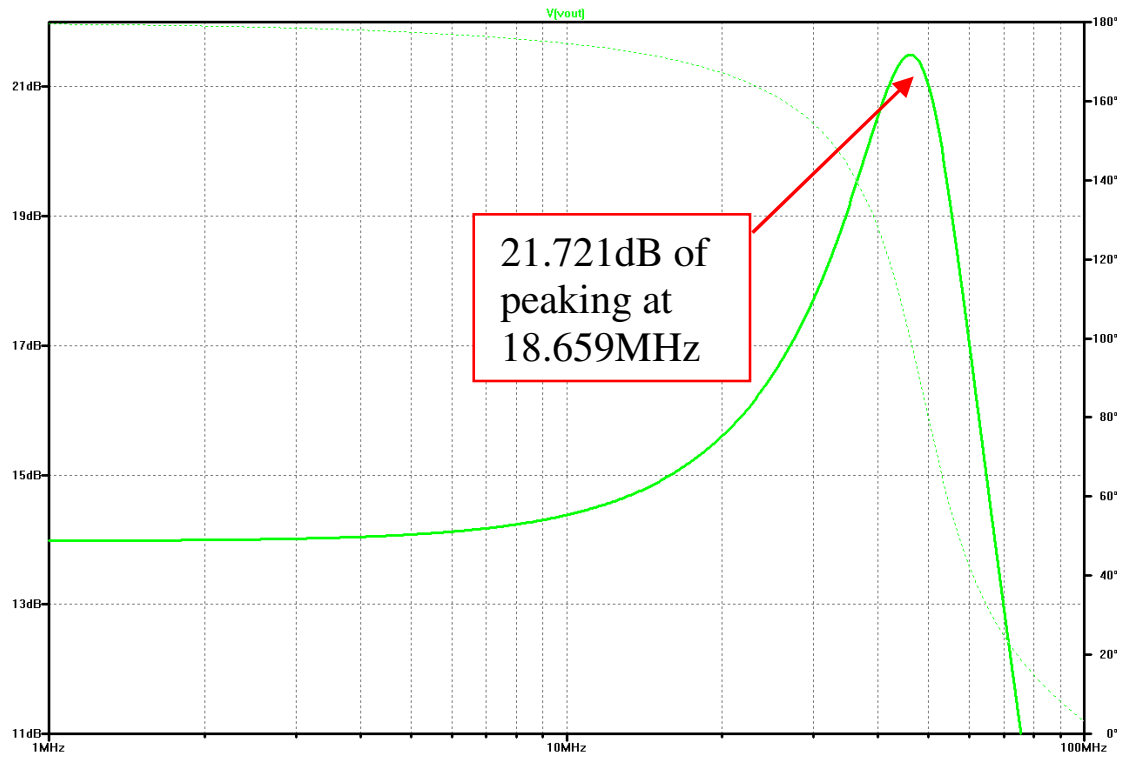

Figure A.15 ? Observable peaking using the suggested amplifier configuration with the LT6200-5.

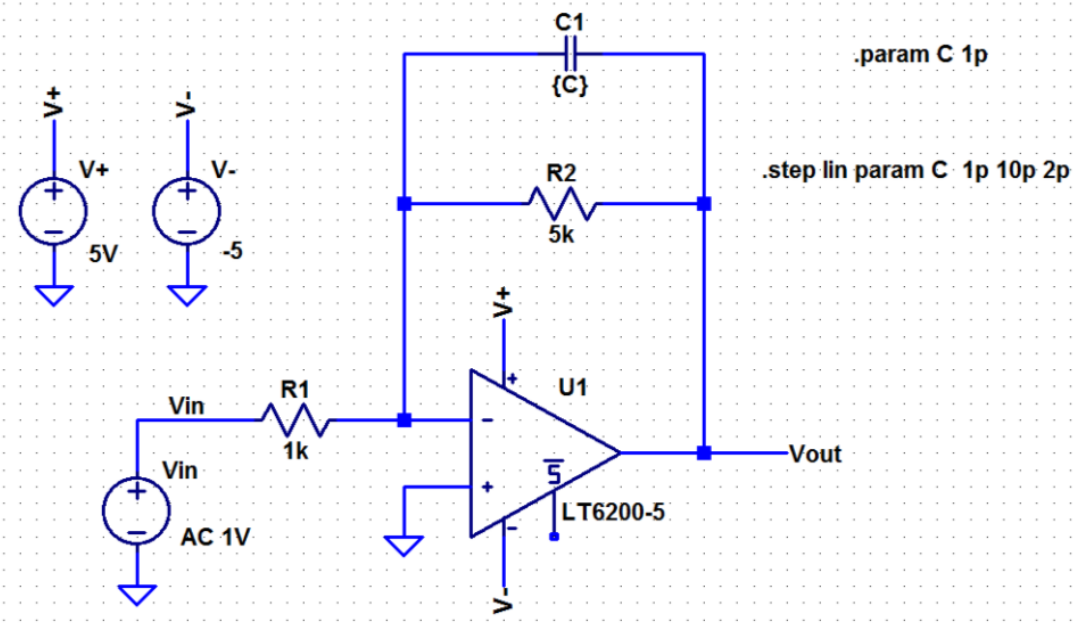

ac dec 1001 100MEG ; tran 10u

Figure A.16 ? Simulated circuit for the LT6200-5 voltage amplifier with swept feedback capacitor values. 


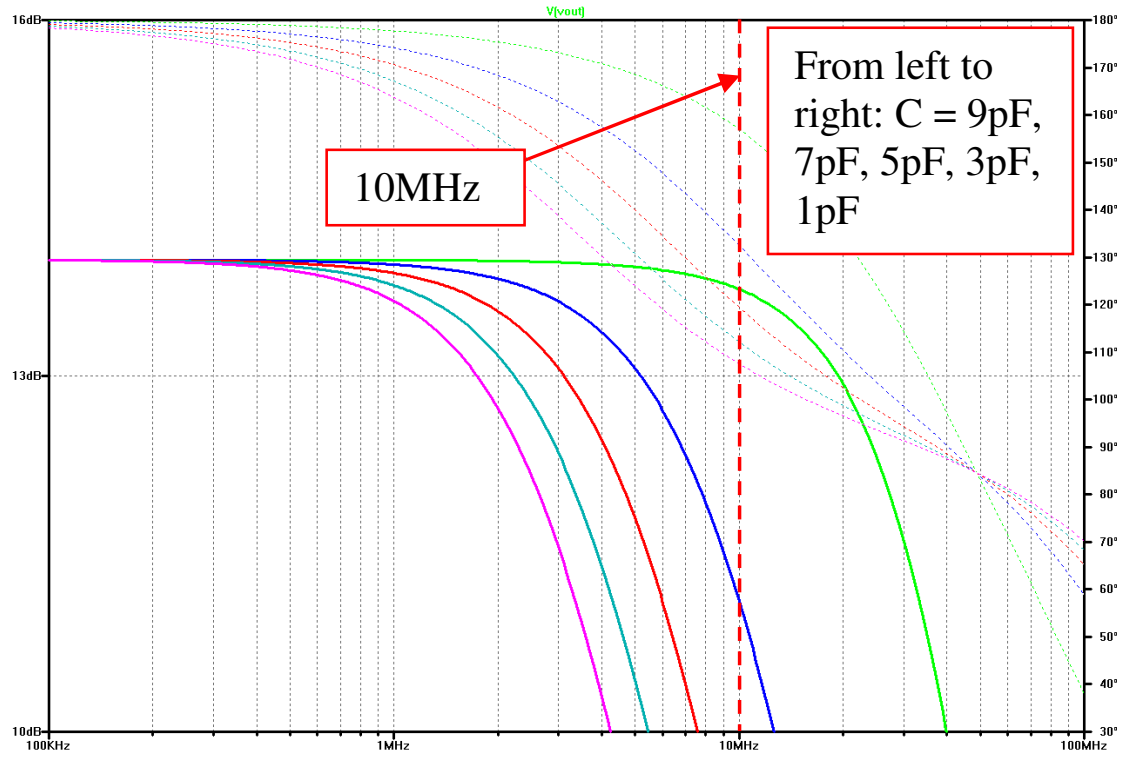

Figure A.17 ? Simulated responses for the LT6200-5 voltage amplifier with swept feedback capacitor values.

A $1 \mathrm{pF}$ feedback capacitor was chosen in the final design of the voltage amplifier.

The circuit for the voltage amplifier stage is shown in Figure A.18 and the resulting response is shown in Figure A.19.

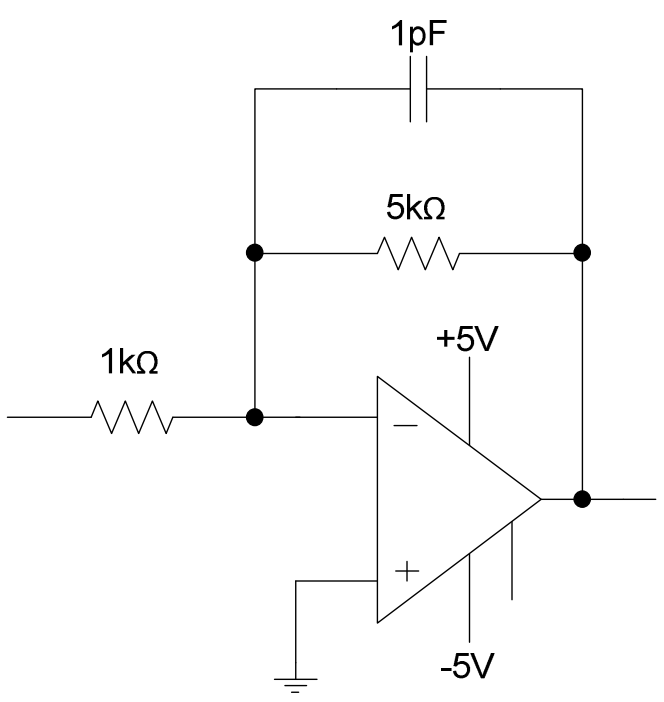

Figure A.18 ? LT6200-5 inverting amplifier configuration. 


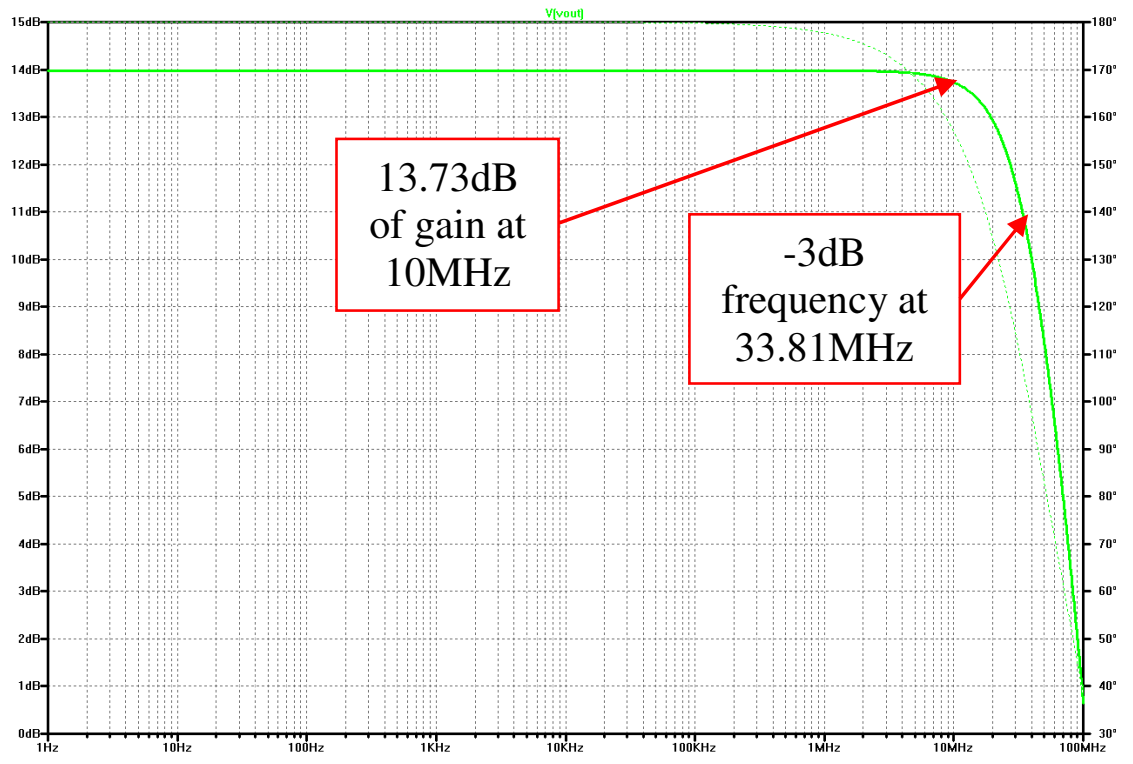

Figure A.19 ? LT6200-5 inverting amplifier response (14dB of gain).

Next, an additional inverting amplifier was added to the previous amplifier. The circuit is shown in Figure A.20 and the overall frequency response is shown in Figure A.21. Note that in the final cascade implementation, a $5.1 \mathrm{k} \Omega$ resistor was chosen in place of each $5 \mathrm{k} \Omega$ due to its availability in metal film form (carbon film resistors are known to produce $1 /$ f noise [52]).

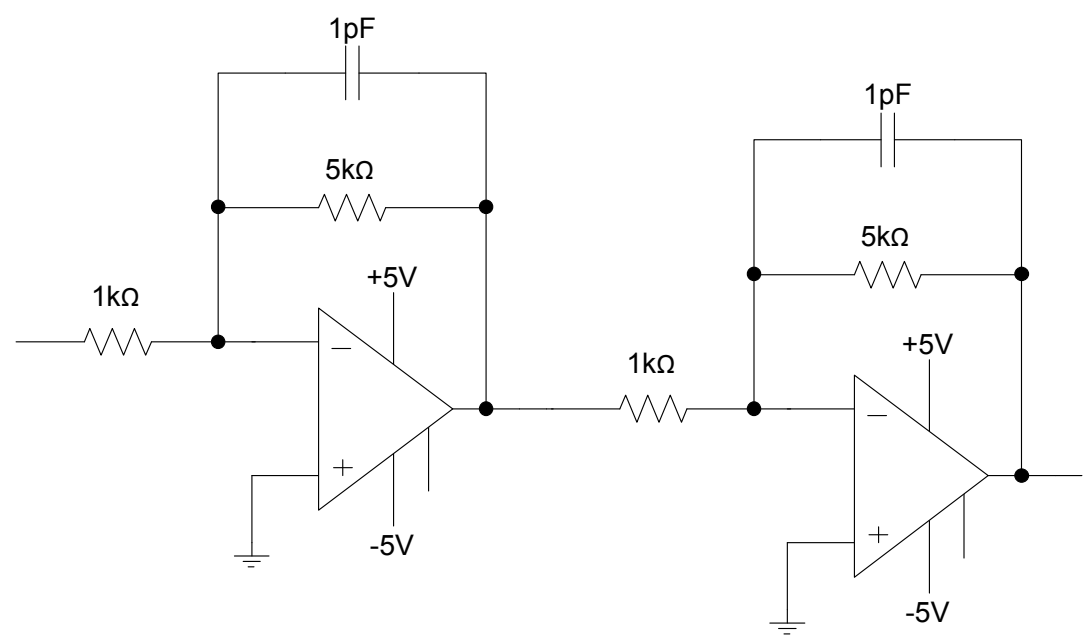

Figure A.20 ? LT6200-5 inverting amplifier cascade. 


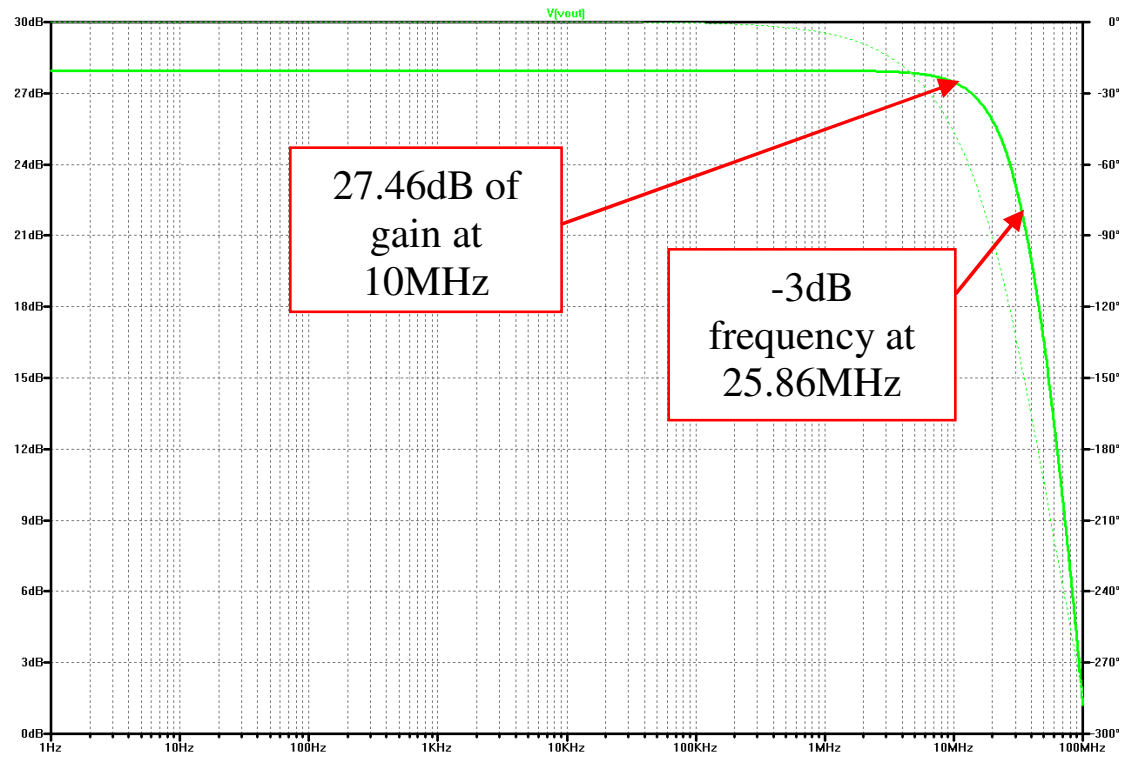

Figure A.21 ? LT6200-5 inverting amplifier cascade response (28dB of gain).

\section{Blocks}

Each of the DC blocks in the system consist of a $2200 \mathrm{uF}$ electrolytic capacitor in parallel with a $0.15 \mathrm{uF}$ capacitor, which creates a relatively lossless block between each stage. The circuit for the DC block is shown in Figure A.22 and the associated frequency response for the can be seen in Figure A.23. Note that the DC block at the drain of the HEMT must have capacitors with voltage ratings of at least $25 \mathrm{~V}$. The DC blocks for the remainder of the system do not require such stringent voltage ratings.

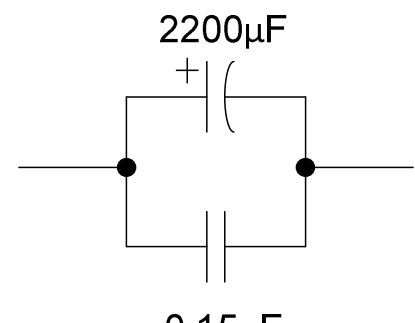

Figure A.22 ? Lossless DC block. 


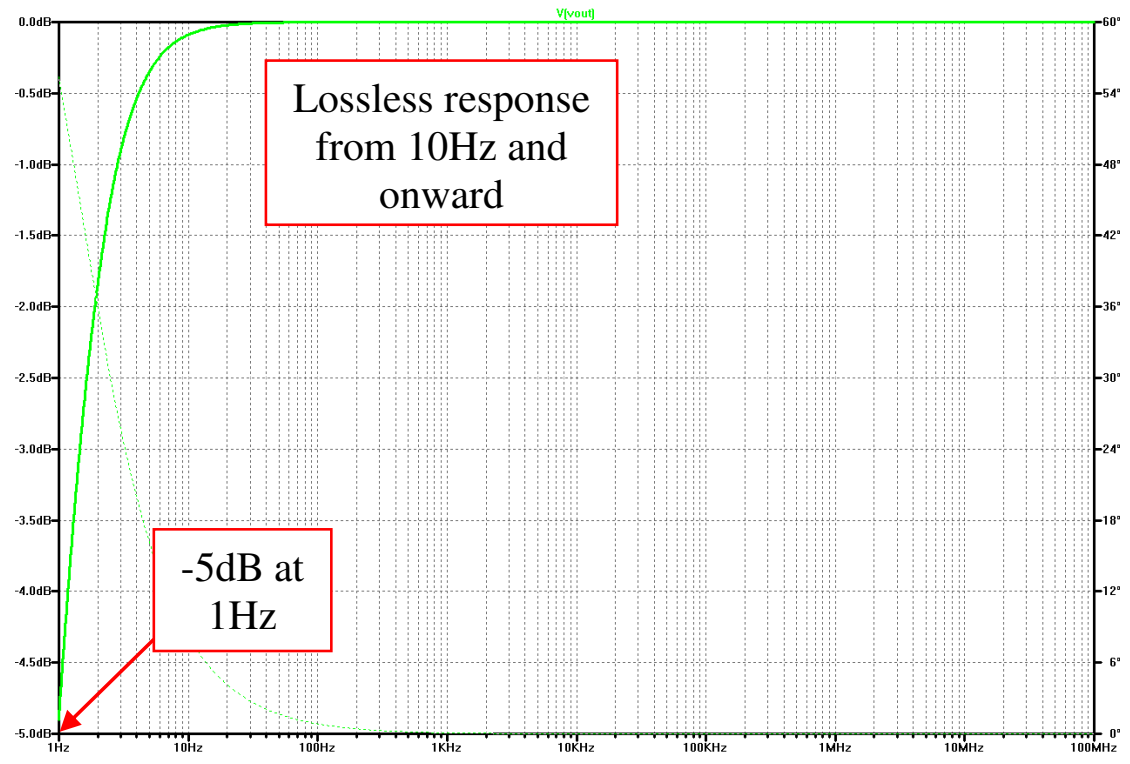

Figure A.23 ? DC block frequency response.

\section{Design Summary}

The final 1/f noise measurement system design is shown in Figure A.24. The associated component values are shown in Table A.4. Note that the system was designed for through-hole parts on a copper-clad perfboard for proto-typing purposes. If the design is able to demonstrate a proof-of-concept, a second iteration will be done on a PCB using higher-quality components. It should be noted that along with certain resistors, specific electrolytic capacitors also contribute to low-frequency noise. Work done in [53] found that the noise contribution from tantalum-based electrolytic capacitors were bias dependent. Therefore, to increase the robustness of the $1 /$ f-noise measurement system, special attention should be made when selecting specific resistors and capacitors in the final design. 


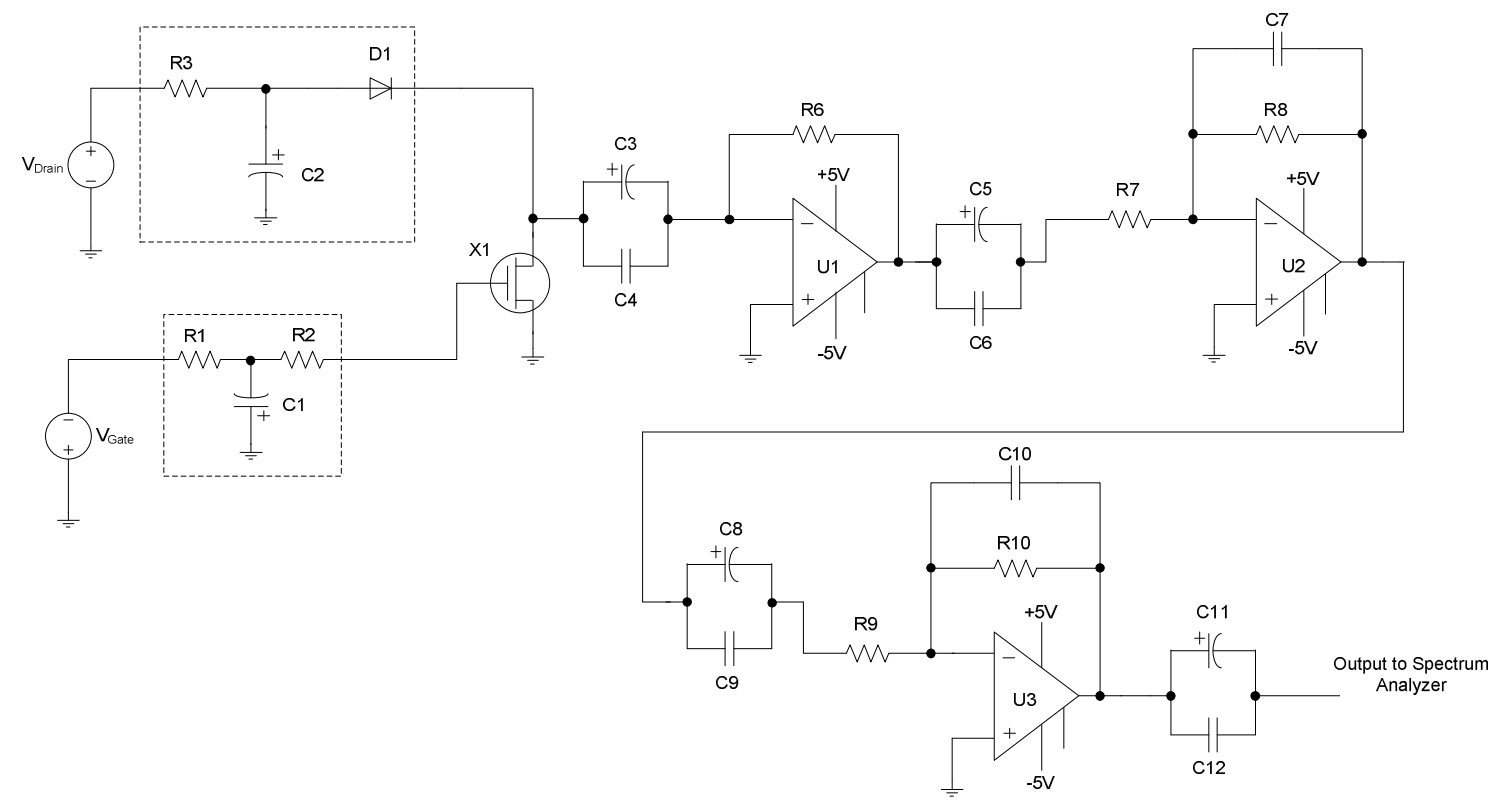

Figure A.24 ?] Final 1/f-noise measurement system design.

Table A.4 [ Component values for the 1/f-noise measurement system design.

\begin{tabular}{|c|c|c|}
\hline Reference & Part Description & Package Type \\
\hline C7, C10 & $1 \mathrm{pF}$, ceramic, $50 \mathrm{~V}, \pm 0.25 \mathrm{pF}$ & Radial \\
\hline $\begin{array}{l}\text { C4, C6, C9, } \\
\text { C12 }\end{array}$ & $0.15 \mathrm{uF}$, ceramic, $25 \mathrm{~V}, 10 \%$ & Radial \\
\hline C3 & $2200 u F$ aluminum electrolytic, $25 \mathrm{~V}, 20 \%$ & $\begin{array}{l}\text { Radial can - } \\
\text { through hole }\end{array}$ \\
\hline C5, C8, C11 & $2200 u$ F aluminum electrolytic, 10V, 20\% & $\begin{array}{l}\text { Radial can - } \\
\text { through hole }\end{array}$ \\
\hline $\mathrm{C} 1$ & $4700 \mathrm{uF}$ aluminum electrolytic, $10 \mathrm{~V}, 20 \%$ & $\begin{array}{l}\text { Radial can - } \\
\text { through hole }\end{array}$ \\
\hline $\mathrm{C} 2$ & $4700 u F$ aluminum electrolytic, 25V, 20\% & $\begin{array}{l}\text { Radial can - } \\
\text { through hole }\end{array}$ \\
\hline R3 & 100hm, 1/4W, 1\% Metal Film & Axial \\
\hline $\mathrm{R} 1, \mathrm{R} 2, \mathrm{R} 4$ & $1000 \mathrm{hm}, 1 / 4 \mathrm{~W}, 1 \%$, metal film & Axial \\
\hline $\mathrm{R} 5, \mathrm{R} 7$ & $1 \mathrm{kOhm}, 1 / 4 \mathrm{~W}, 1 \%$, metal film & Axial \\
\hline R6, R8 & $5.1 \mathrm{kOhm}, 0.40 \mathrm{~W}, 1 \%$, metal film & Axial \\
\hline D1 & 1N4001 general purpose rectifier & Through-hole \\
\hline U1, U2, U3 & $\begin{array}{c}\text { LT6200 - 165MHz, Rail-to-Rail Input and Output, } \\
0.95 \mathrm{nV} / \mathrm{VHz} \text { Low Noise, Op Amp Family }\end{array}$ & SOIC-8 \\
\hline $\mathrm{X} 1$ & NPTB00004 5W GaN HEMT & $\begin{array}{l}\text { SOIC-8 (with } \\
\text { source pad) }\end{array}$ \\
\hline
\end{tabular}




\section{Appendix B: Cadsoft Eagle Tutorial}

\section{PCB Design: Package Creation}

Download the free version of Cadsoft Eagle from http://www.cadsoftusa.com/download-eagle/?language=en. There are usually three steps involved in the entire PCB process: package creation, schematic entry, and PCB layout. The package creation step, in some cases, can be skipped if the package/footprint for a specific part is already given.

Start by creating a new library in Eagle (refer to Figure B.1). After a new library window appears, name the library file and save it to the "lbr" directory where Eagle was installed to (example: JohnDoeCustom.lib).

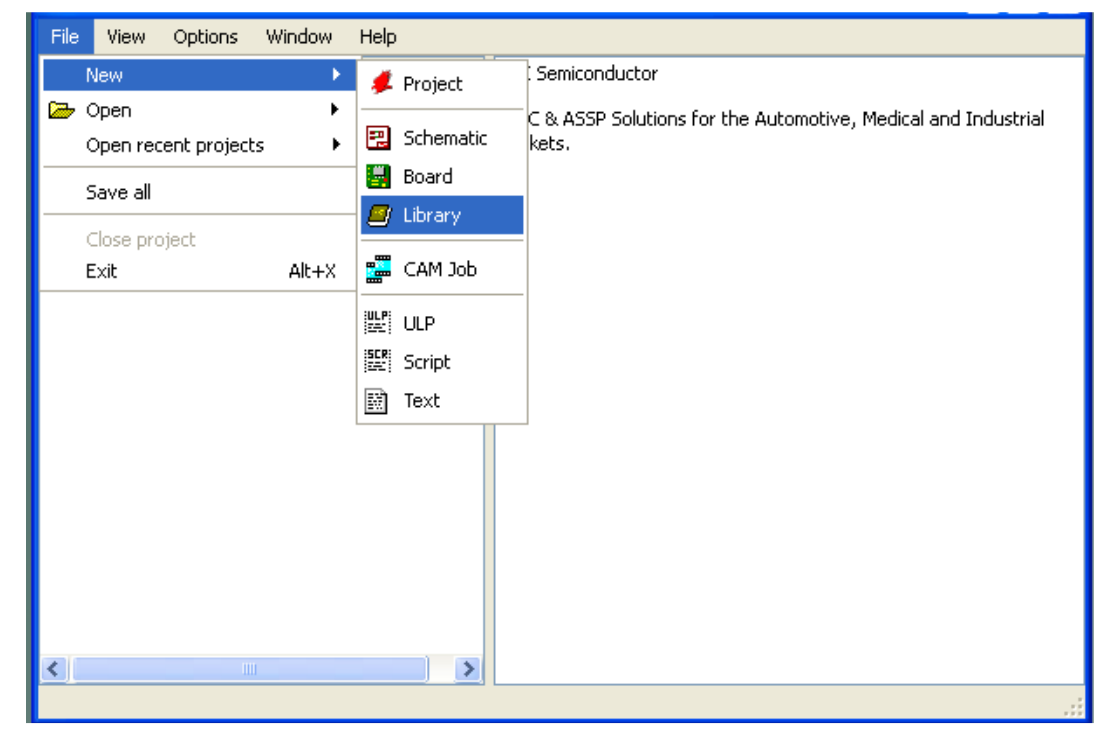

Figure B.1 [ Cadsoft Eagle main window - starting a custom library.

During the component creation process, there are three steps the user must proceed through to make the final part. The three steps are Package Creation, Symbol Creation, and Device Creation. Package Creation involves creating a footprint for the component, which will consist of laying out land patterns (surface mount, through-hole, 
etc.), silk screens, and so forth. Symbol Creation involves creating the schematic symbol for the component. Component Creation links the package to the schematic symbol. Refer to Figure B.2 for the library window.

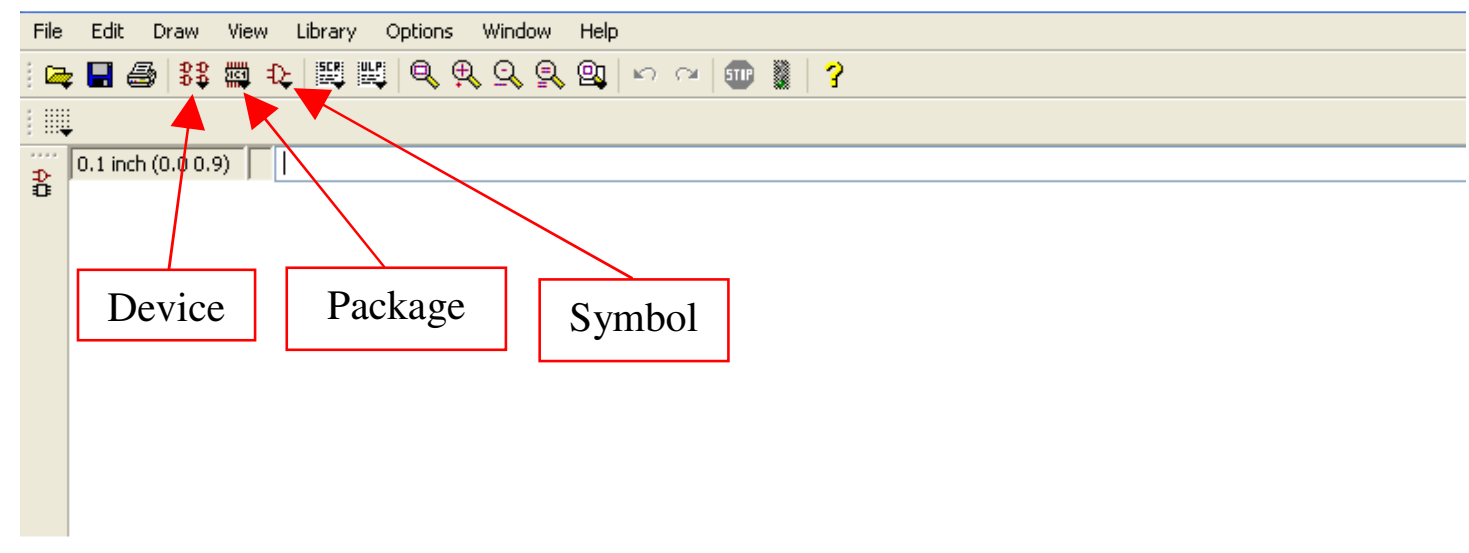

Figure B.2 ? Cadsoft Eagle library window.

Begin component creation by selecting the package button. When the "Edit" window appears, name the package after the component's package type, and then save the name. A black background with a grid should appear; the window has now changed to the package creation window. Before setting up the package in the workplace, the user should have consulted the component's datasheet for recommended land patterns. Once the land pattern is found, start the process by setting up the work space's grid (Figure B.3). After setting a desired grid area, start placing either surface mount pads (SMDs) or through-hole pads onto the workspace using the recommended land patterns from the datasheet. The size and shape of each pad can be altered by going to the top of the work space and changing the dimensions (Figure B.4). To make a circular SMD, set the roundness to $100 \%$. 


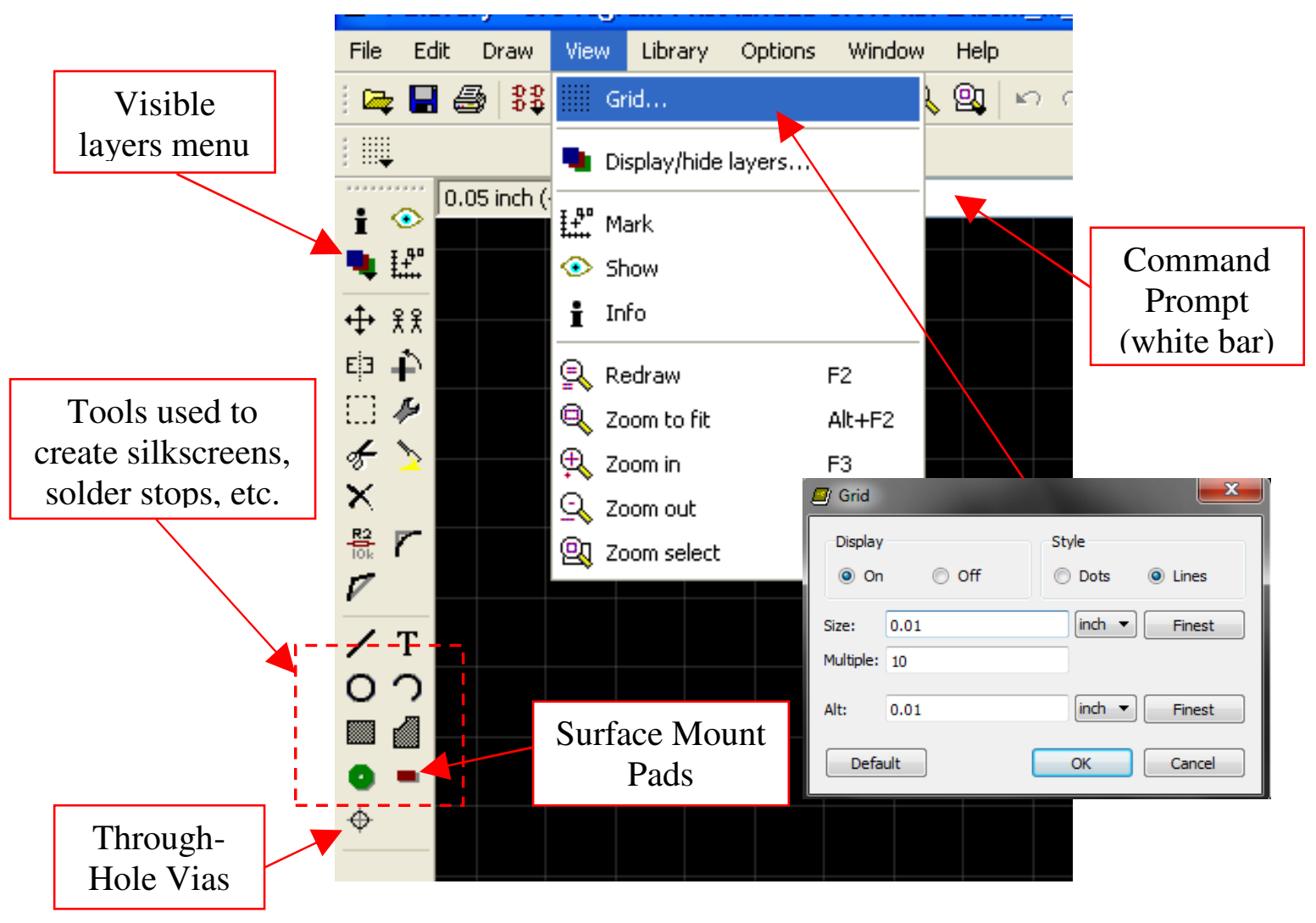

Figure B.3 ? Package creation work space with typical grid setting.

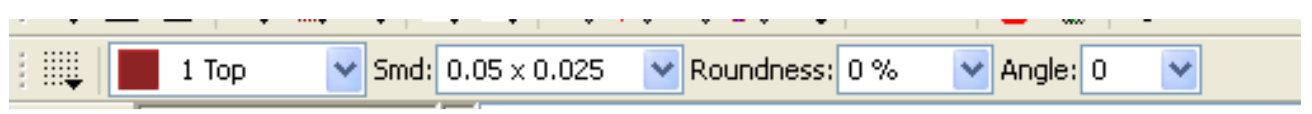

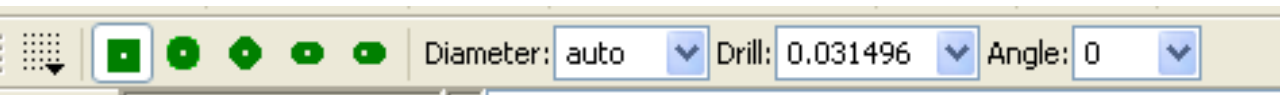

Figure B.4 ? SMD and through-hole size/dimension options, respectively.

Note that the SMDs should be on the "Top" (copper) layer, and the through-hole pads should be on the "Pads" layer. To check if certain aspects of the design are on the right layers, select the visible layers drop down and select/deselect layers. After placing pads, silkscreens can be placed to outline the component's package and display the component's schematic reference number and manufacturer number. Component outline silkscreens can be created using the Wire and Circle tools on the left toolbar, while the 
reference number (Name) and manufacturer's part number (Value) silkscreens can be created using the Text tool. Note that during component creation, the reference number and part number should be set as ">Name" and ">Value." The actual designators will be placed onto the layout workspace once the schematic is created. The component outline silkscreens should be created on the "tPlace" layer while the Name and Value silkscreens should be placed on the "tName" and "tPlace" layers, respectively. The " $t$ " in each layer represents the top layer ("b" would then represent the bottom layer). As an example, a finished part is shown in Figure B.5 (8-pin DIP socket for the TL072).

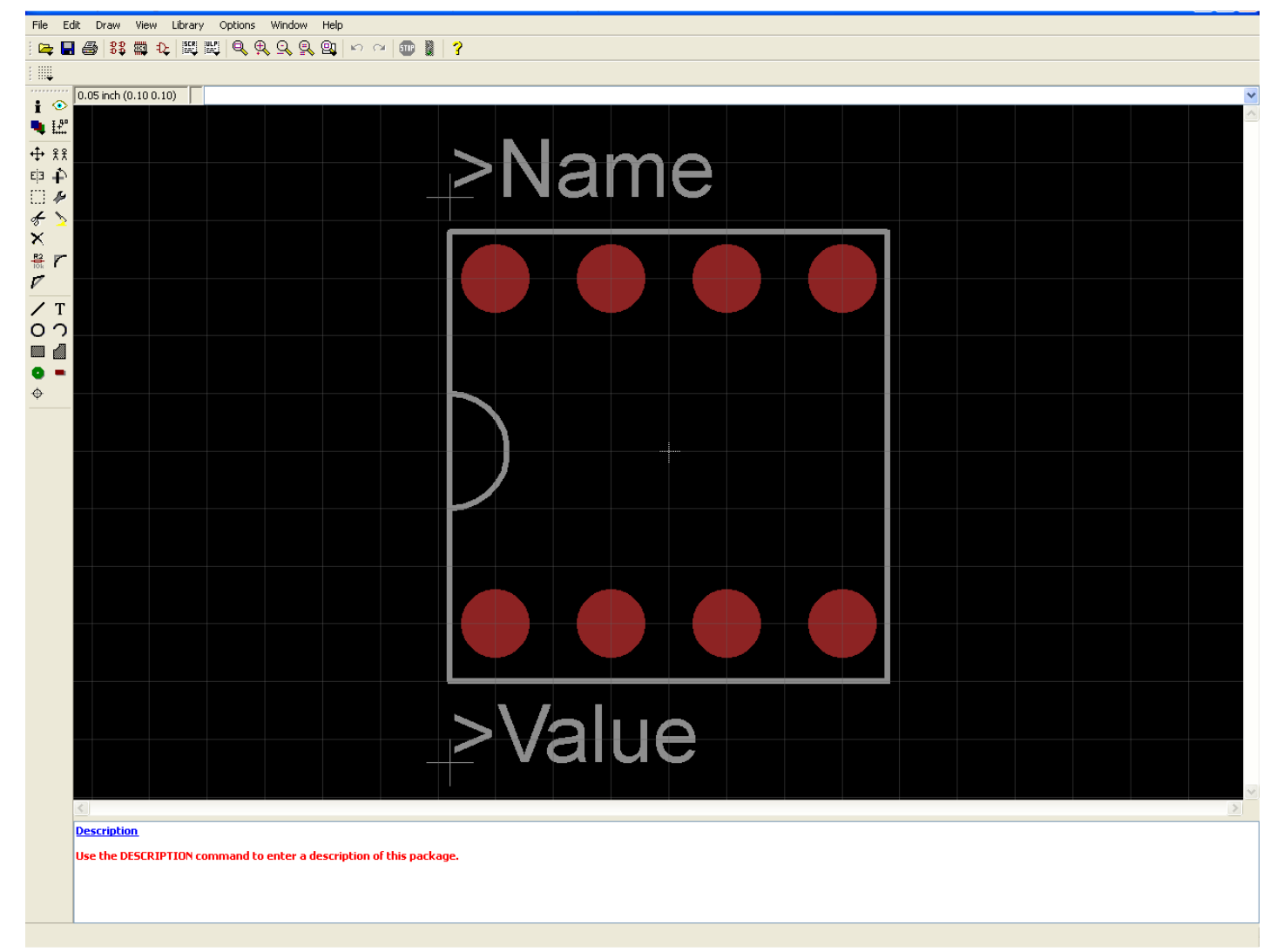

Figure B.5 ? Package design example - 8-pin DIP socket for the TL072.

A few tips about the package creation process should be mentioned. The first is the shortcut to place pads down through coordinate commands. At the top of the work 
space is a white command prompt bar. Instead of clicking and dragging pads to a specific coordinate, each point can be entered in an (x y) format. For example, if a pad is required 60mils x 100mils from the origin (and the units are in inches), the coordinate command would be (0.060 0.100) (type the coordinates and press enter).

The second tip revolves around the use of the crosshair at the center of the workspace. The crosshair is used as an indicator for how a part will be placed onto the user's cursor when being dragged and placed onto the workspace during the layout stage. Therefore it is a matter of preference for the user on how the part will be oriented onto the workspace during the package stage. A general method used is to center the bottomleft-most pad onto the cross-hair and design the package from there. Once the package is completed, select the Group tool and drag a selection box over every single package element. Once the package is highlighted, select the Move tool and right-click near the package. At the bottom of the tool-tip that shows up, select Move: Group. Center the package around the center cross-hair and save the design.

The third tip is in regards to pad naming. To make connections between the schematic symbol and the package simple, each pad can be given a name. Type "Name" into the command prompt and select each pad. A new window will come up where the respective pin name or designator can be edited. The same process should be performed during schematic symbol creation when handling pin names.

Next, create a schematic symbol by selecting the associated icon. An "Edit" prompt will appear much like during the package process. Name the schematic symbol after the manufacturer's part name and proceed. A window similar to the package creation work space will appear, but the background will be white (Figure B.6). The 
default grid configuration should be acceptable for this process, but can also be altered to the user's preferences. The entire schematic process is similar to that of the package process, where each pin will receive a name/designator and the symbol will be labeled with the $>$ Name and $>$ Value conventions. An example symbol can be seen in Figure B.7 (8-pin DIP socket for the TL072).

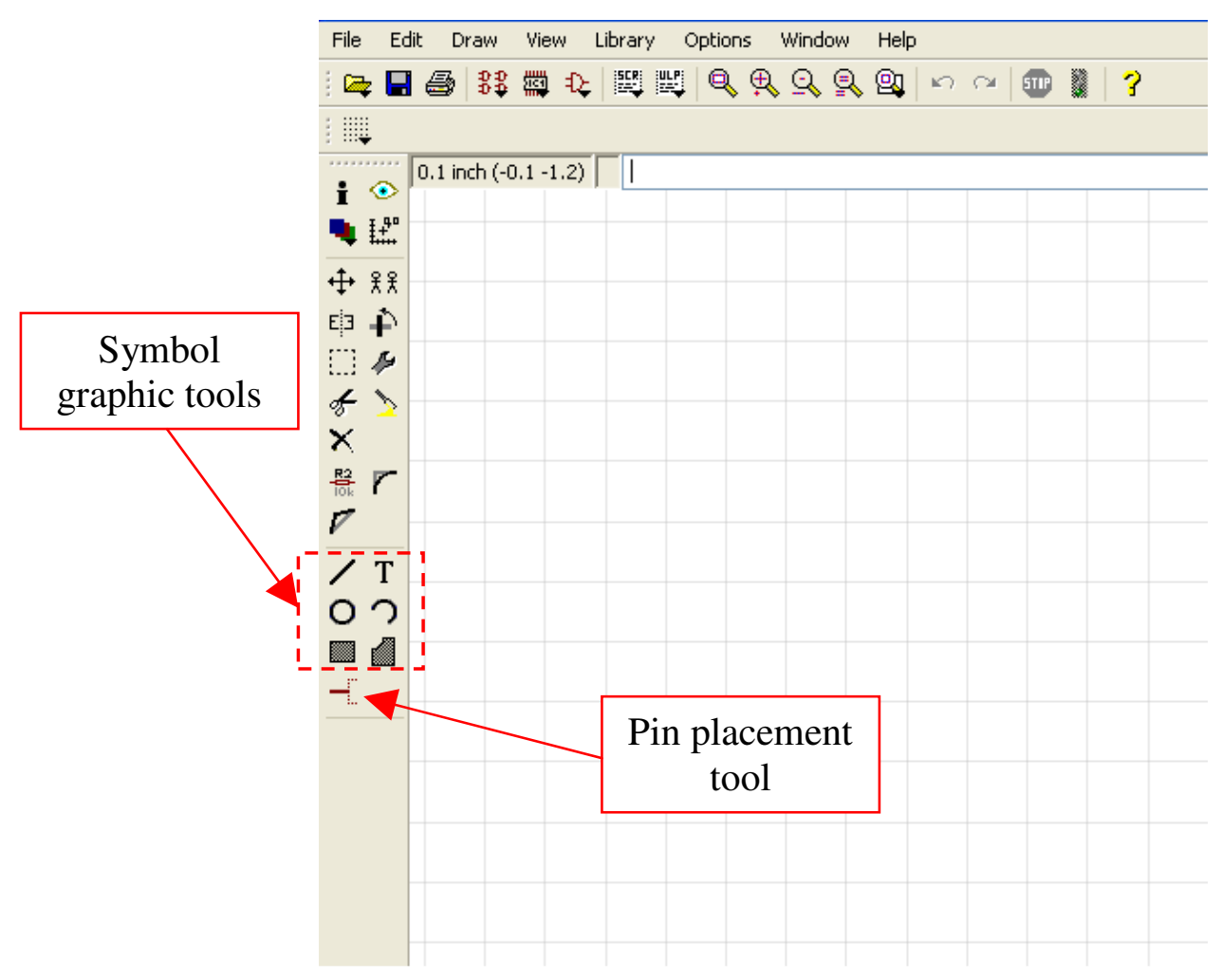

Figure B.6 ? Schematic symbol creation work space. 


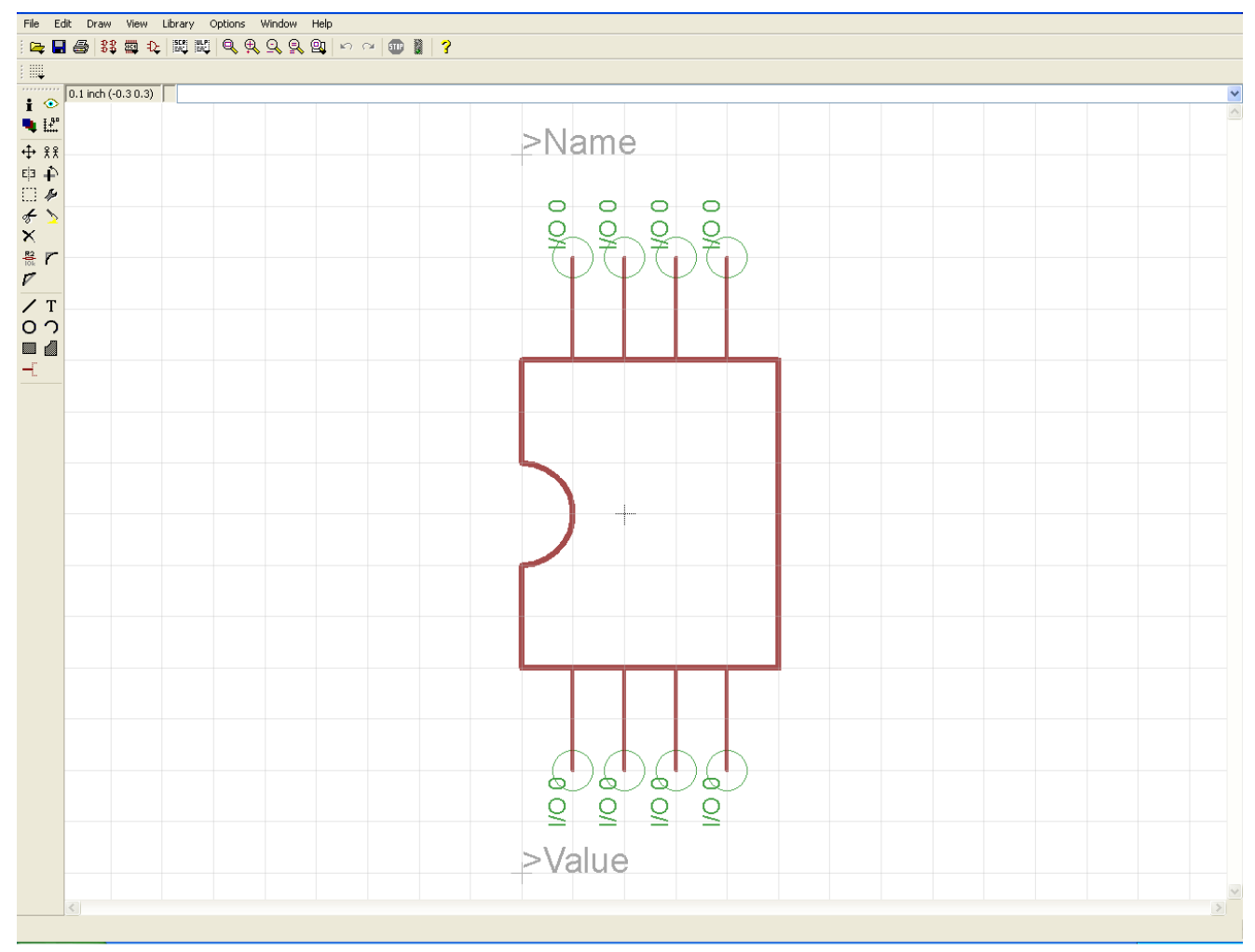

Figure B.7 [ Schematic symbol design example - 8-pin DIP socket for the TL072.

Finally, select the Device icon to complete the component creation process. A new window should appear as seen in Figure B.8. First, click the Add button and select the schematic symbol that was created. Next, click the New button and add the created package symbol. Finally, press the Connect button to link the schematic and package symbols together. The connection window should look like Figure B.9. If the pads and pins from the previous processes were given their respective names, they should line up directly with one another as seen in Figure B.9. If not, the user will have to search for and select the connections between each associated pad and pin. By the end of the connection process, the "Connection" section in the last-third of the window should be full. Press "OK" to complete the component process. 


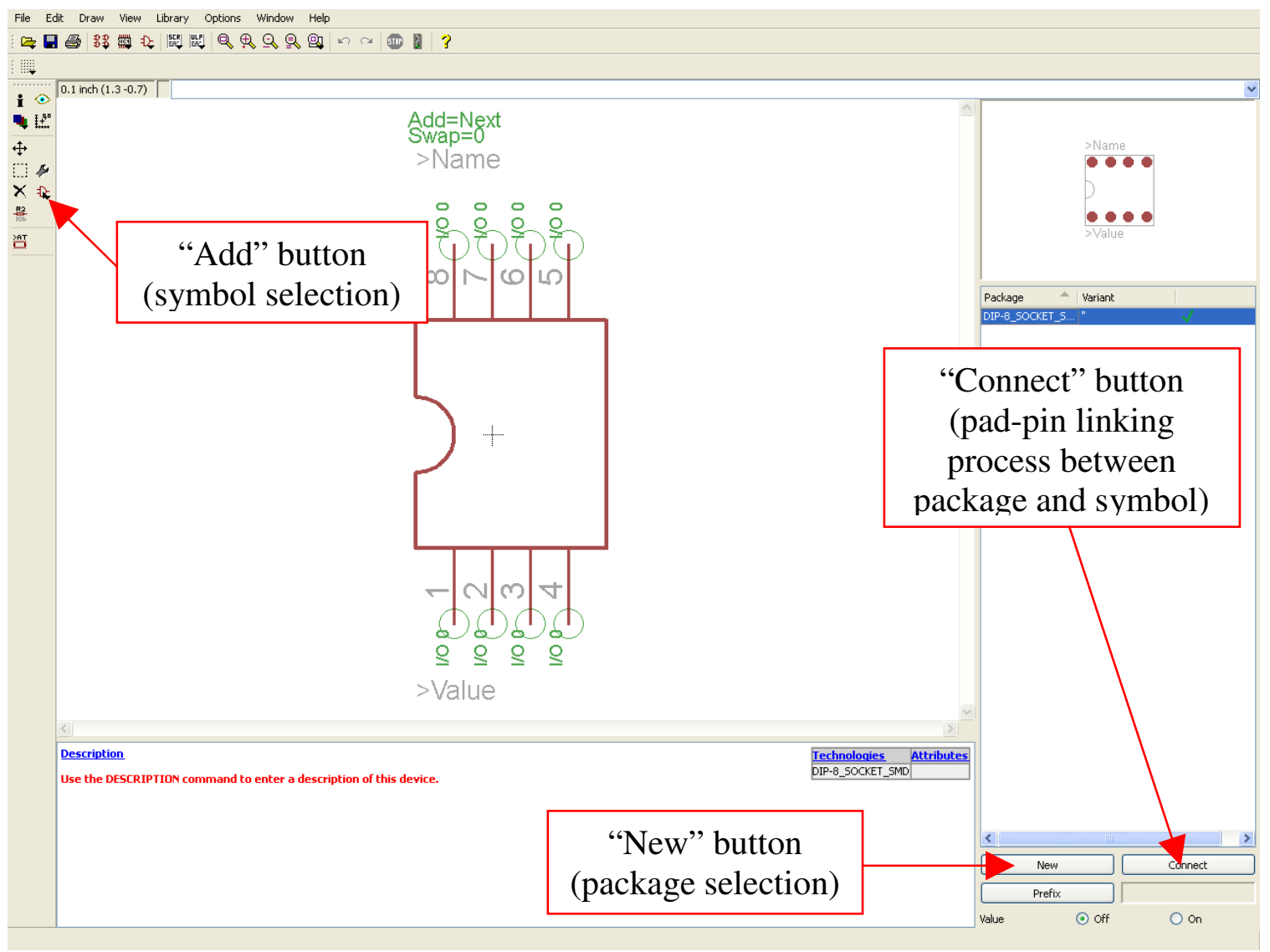

Figure B.8 ? Device creation work space.

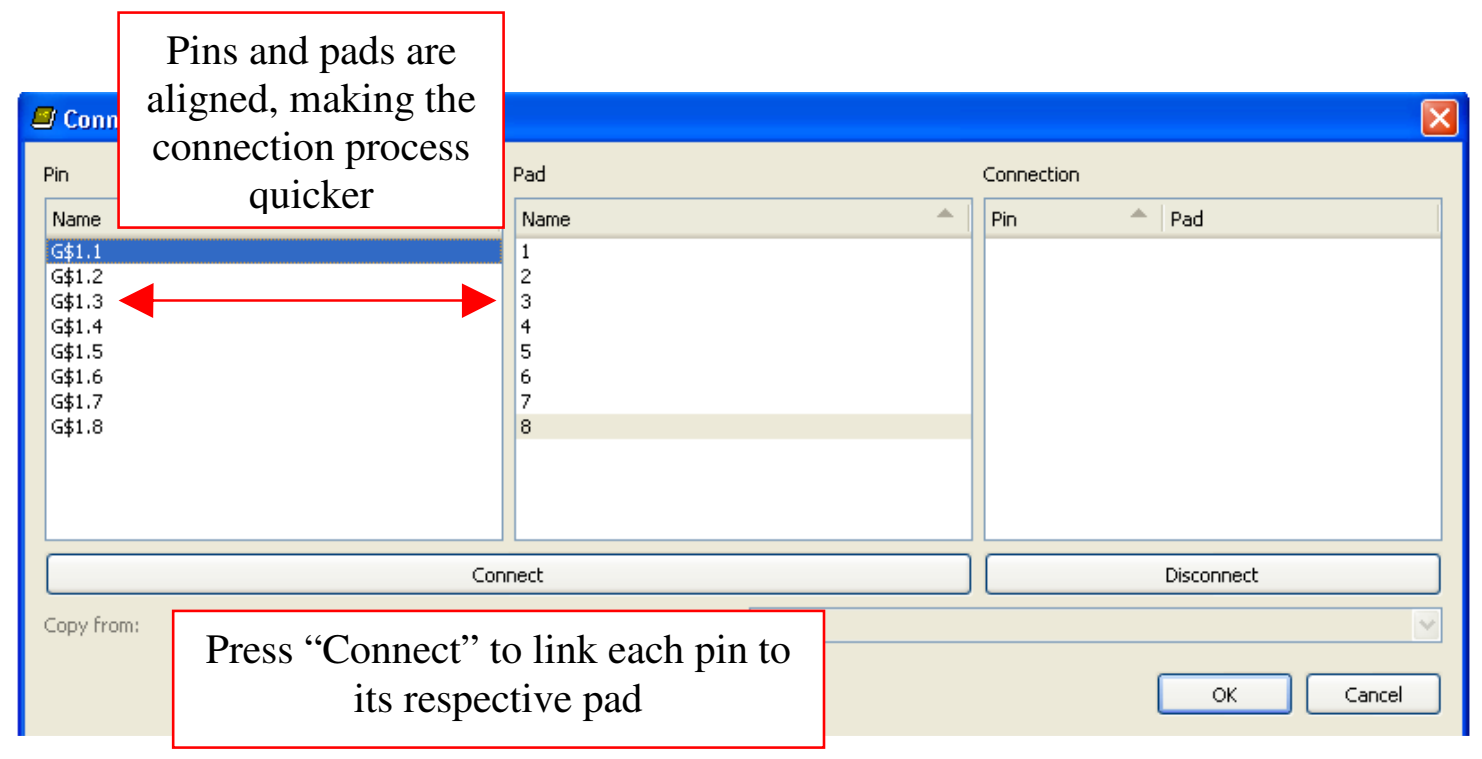

Figure B.9 ? Schematic and package symbol connection window. 


\section{PCB Design: Schematic and Layout}

Start a new project in Eagle by referring to Figure B.10, and then start a new schematic as seen in Figure B.11.

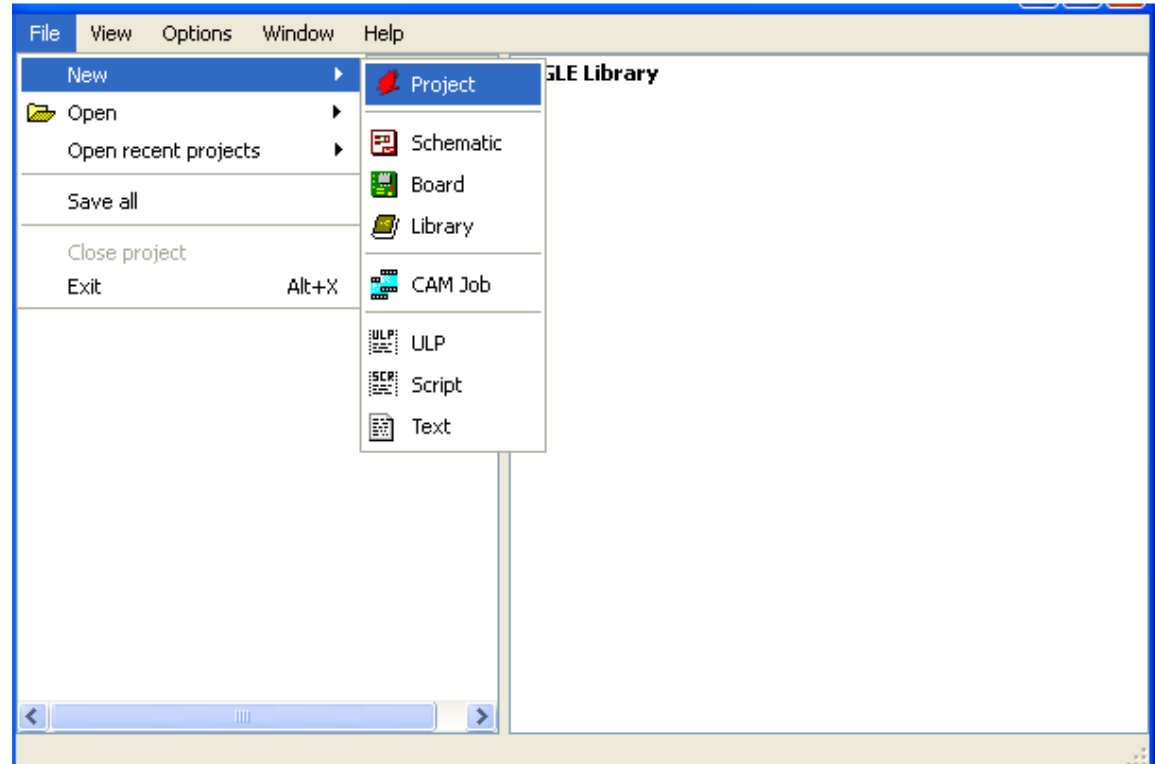

Figure B.10 ? Starting a new project in Eagle.

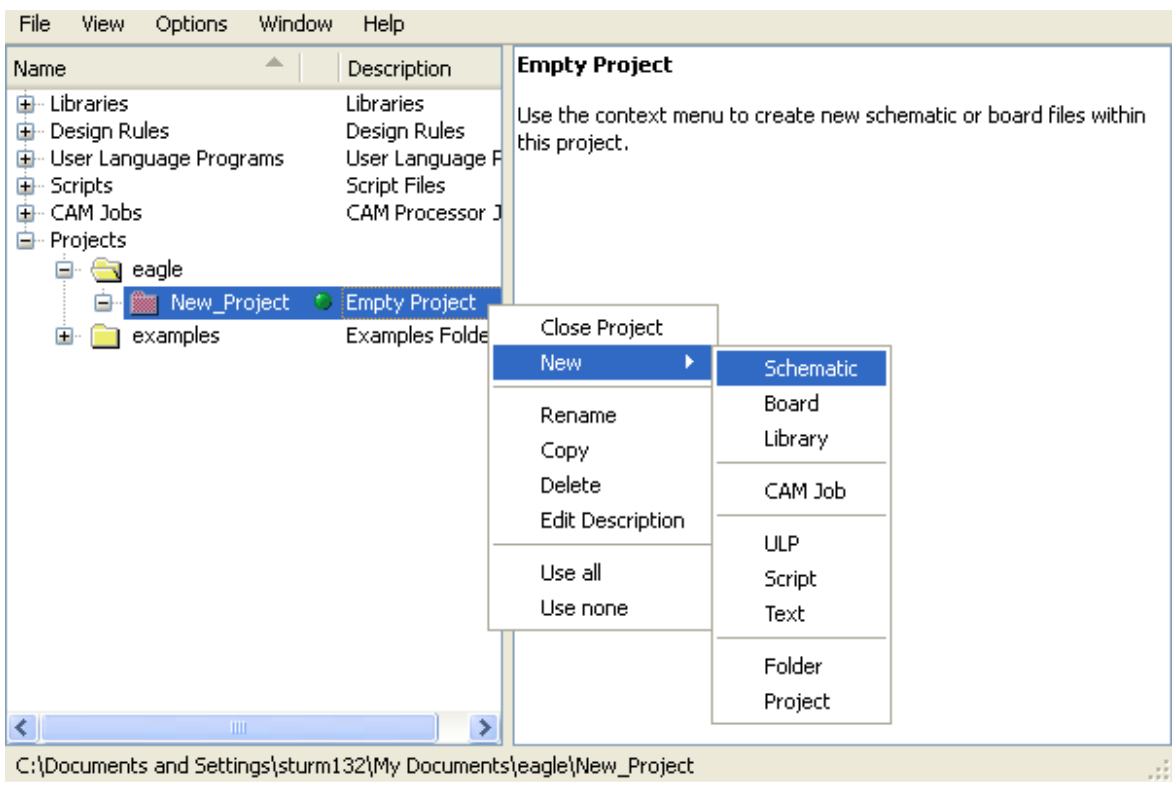

Figure B.11 ? Starting a new schematic in the project directory. 
A new schematic work space should appear, as seen in Figure B.12. All parts should be accessible via the $\boldsymbol{A} \boldsymbol{d} \boldsymbol{d}$ button (including parts from the custom-made library). Place parts onto the schematic work space and wire them together using the Wire tool. All components can have their reference names and values changed by selecting the Name and Value tools. One useful tool to use is the Group tool when having to move or copy multiple components at any given time. For example, group all desired components together by creating a selection box with the Group tool. Once all desired components are highlighted, select either the move or copy option, and then right-click the work space. Finally, when the tool-tip appears, select "Group: "<Action>," where $<$ Action $>$ is either move, copy, or another command. After completing the schematic entry, press the Board button at the top of the work space to generate a board layout from the schematic.

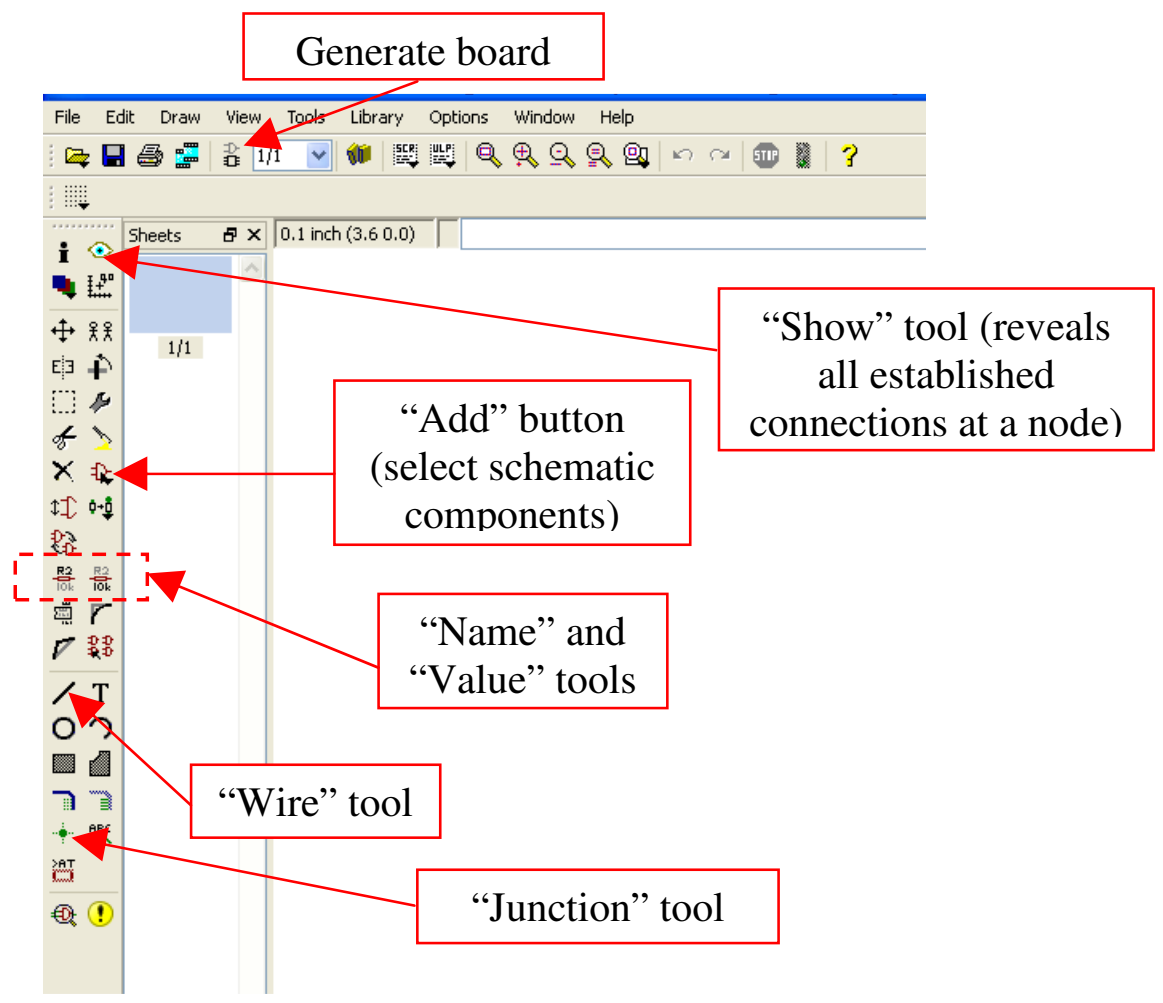

Figure B.12 ? Schematic workspace. 
When Eagle generates the board layout, the program will create a component pile at the bottom left corner of the layout work space (Figure B.13). Before routing the board, it is usually wise to specify the design rules for the board, which follow the guidelines established by the board fabricator. To access the design rules, go to Tools > DRC... and input the necessary fabrication limitations.

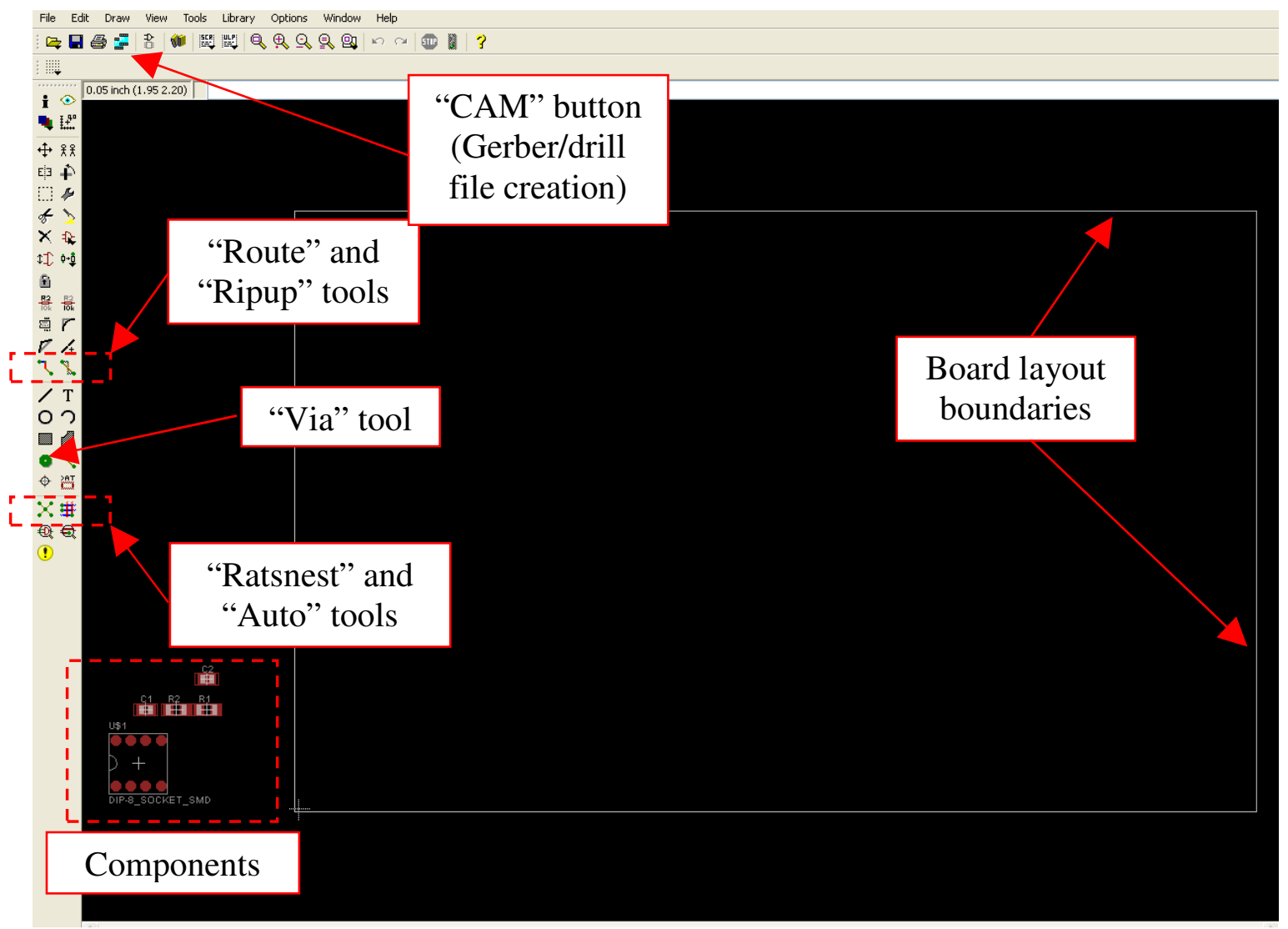

Figure B.13 ? Layout workspace.

As seen in Figure B.13, the white rectangular boundaries within the work space are the board dimensions for the PCB. The boundaries can be adjusted by clicking and dragging the polygon. Each of the components on the screen can also be clicked and dragged onto the PCB area. Note how each connected component from the schematic is now connected by a yellow line known as a "netlist." All components can either be 
manually routed by following each netlist, or an auto-route feature can be used by selecting the Auto button on the left toolbox. When routing components, a majority of the routing will take place on the "Top" layer (rustic-colored). However, routing can also take place on the "Bottom" layer (blue-colored). For multi-layer designs, such as a 4layer board, the top and bottom layers are usually the signal layers, whereas the inner layers are usually dedicated to power and ground. The 2-layer board in this tutorial, however, with utilize the top layer as the component layer and the bottom layer as ground.

To set the bottom layer as ground, select the Polygon tool in the left toolbox, and then select the layer to be "Bottom" from the layer pull-down menu at the top of the work space. Create a rectangular polygon around the inner sides of the PCB boundary (keep a small amount of buffer room between the ground plane and board edges). After the polygon is created, right-click on the polygon, select "Properties," and rename the signal name to "GND." Once connections are made to the GND netlist, the bottom layer should automatically fill up from the blue polygon GND layer. If not, press the "Ratsnest" button. The same methodology can be used to create polygons dedicated to "power" netlists as well.

\section{PCB Design: Gerber File Generation}

To generate the Gerber and drill files, select the "CAM" button at the top of the work space (refer to Figure B.13). When the CAM processor window opens, a job must be selected to generate the Gerber files for a 2-layer board (refer to Figure B.14). Next, select the "gerb274x.cam" job file, which is a default job created specifically for 2-layer boards. 


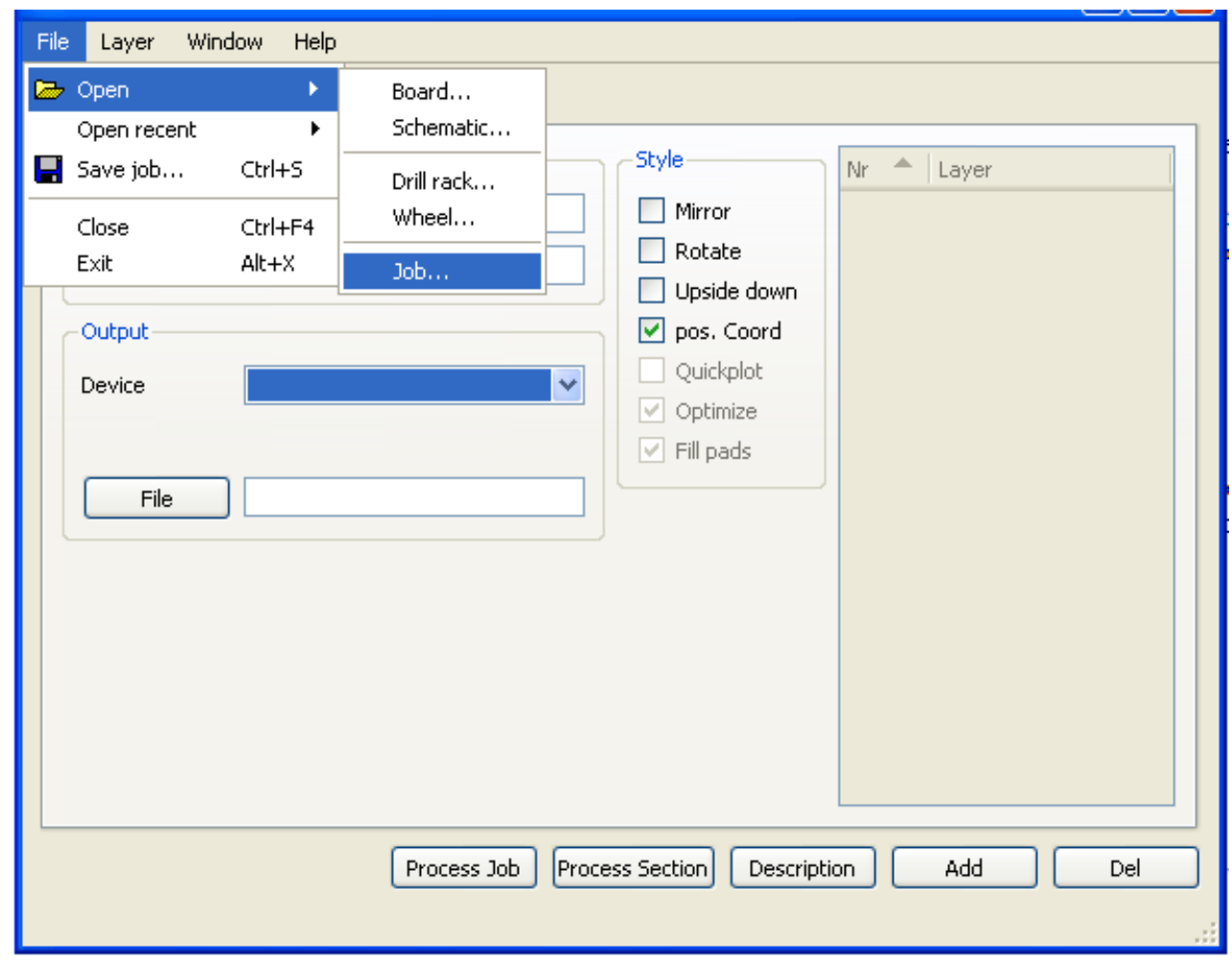

Figure B.14 ? CAM processor window.

Once the job is selected, the Cam processor window should now look like Figure B.15. Take notice of the tabs that appear at the top of the window. Gerber files should be generated for the component layer, ground layer, component silkscreens, component layer solder-mask stop, and ground layer solder-mask stop. For each layer, the affected board attributes are highlighted in blue. Click "Process Job" when all parameters are configured. 


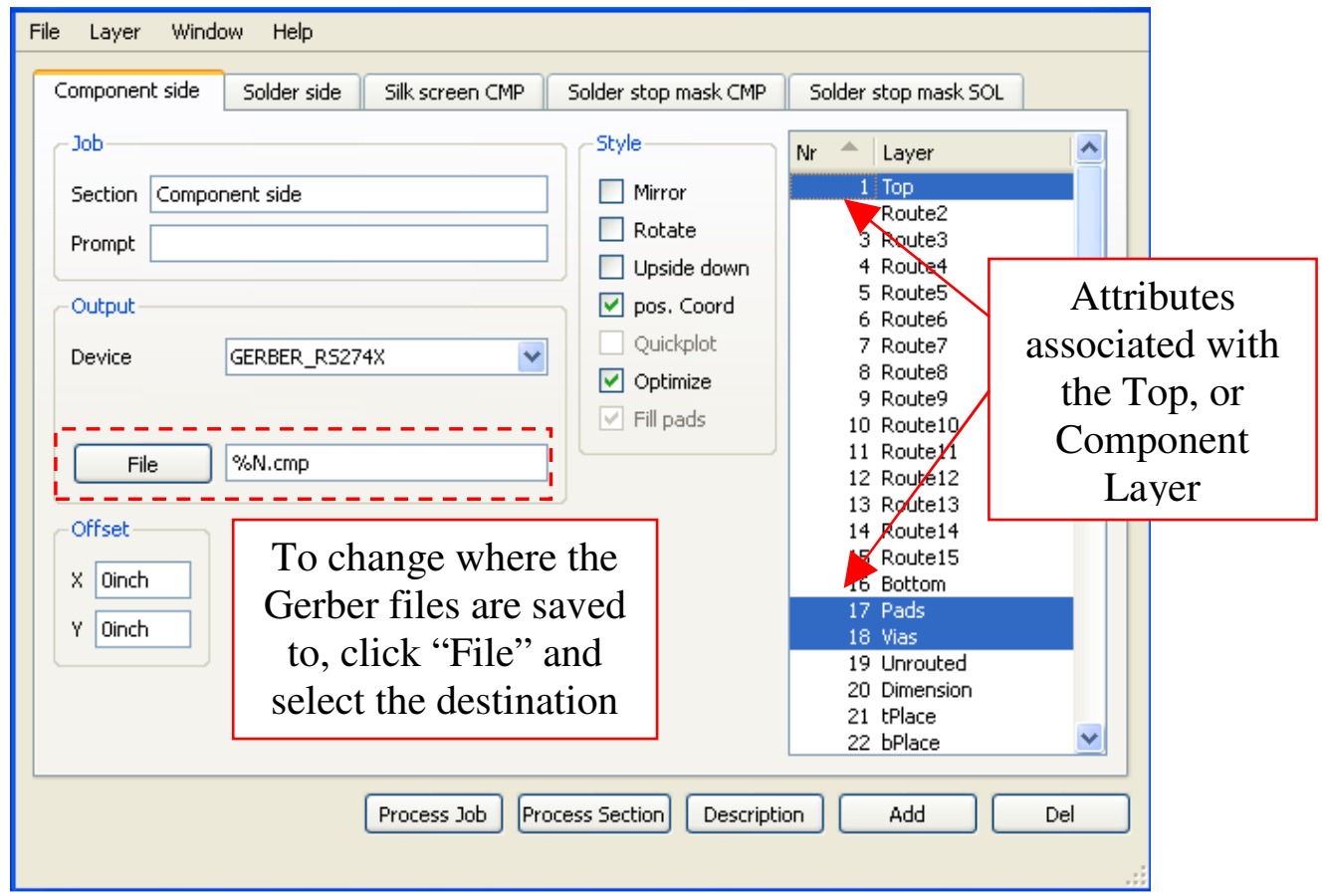

Figure B.15 ? Gerber file configuration for a 2-layer board job.

After the Gerber Files are saved, select File $>$ Open $>$ Job... and select the "excellon.cam" job to create the drill files for the board. A similar screen like Figure B.15 should appear. Again, once all parameters are set, click "Process Job." When sending the Gerber and drill files to a board fabrication house, certain files are necessary in the board fabrication process. For this project, the company Advanced Circuits (www.4pcb.com) was used as a board fabrication vendor. Table B.1 lists the files necessary to have Advanced Circuits fabricate a board. 
Table B.1 ] Necessary Gerber and drill files for board fabrication.

\begin{tabular}{|c|c|}
\hline File & Description \\
\hline .cmp & Component-side (top) data \\
\hline sol & Solder-side (Bottom) data \\
\hline .plc & Component-side silk screen data \\
\hline stc & Component-side solder stop-mask data \\
\hline .sts & Solder-side stop mask data \\
\hline drd & Excellon drill description \\
\hline
\end{tabular}

